for

\title{
Visible-Light-Mediated Hydroacylation of Azobenzenes with $\alpha$-Keto Acids
}

\author{
Jingya Yang, ${ }^{* \dagger}$ Menghui Song, ${ }^{\dagger}$ Hongyan Zhou, ${ }^{\dagger+\dagger}$ Ganggang Wang, ${ }^{\dagger}$ Ben Ma, ${ }^{\dagger}$ Yuanyuan Qi, ${ }^{\dagger}$ and \\ Congde Huo*, \\ ${ }^{\dagger}$ College of Chemistry and Chemical Engineering, Northwest Normal University, Lanzhou 730070, \\ China \\ ${ }^{\ddagger}$ College of Science, Gansu Agricultural University, Lanzhou 730070, China \\ E-mail: yangjy@nwnu.edu.cn; huocongde1978@hotmail.com
}

\section{List of Contents}

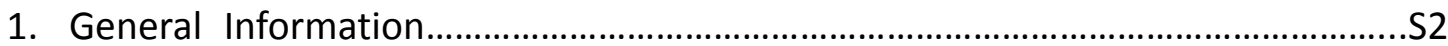

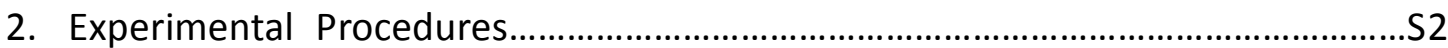

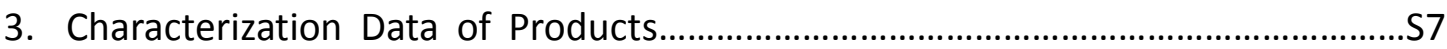

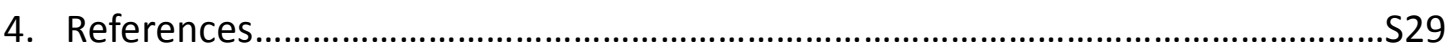

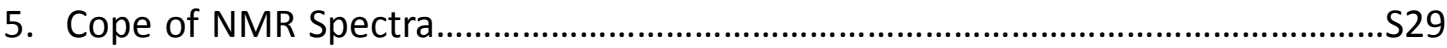




\section{General Information}

${ }^{1} \mathrm{H}$ NMR, ${ }^{13} \mathrm{C}$ NMR and ${ }^{19} \mathrm{~F}$ NMR spectra were recorded on an Agilent Technologies DD2 (600 $\mathrm{MHz}$ ) or a Varian Mercury-400 Plus spectrometer in $\mathrm{CDCl}_{3}$ or DMSO- $d_{6}$. Chemical shifts $(\delta)$ for NMR were quoted in ppm relative to the solvent peak (7.26 ppm for ${ }^{1} \mathrm{H}$ and $77.00 \mathrm{ppm}$ for ${ }^{13} \mathrm{C}$ in $\mathrm{CDCl}_{3} ; 2.49 \mathrm{ppm}$ for ${ }^{1} \mathrm{H}$ and $39.5 \mathrm{ppm}$ for ${ }^{13} \mathrm{C}$ in DMSO- $d_{6}$ ). Coupling constants $J$ are recorded in $\mathrm{Hz}$. High-resolution mass spectra (HRMS) were reported from the Thermo Orbitrap Elite or Bruker Daltonics APEXII 47e FT-ICR instrument with an ESI source. UV-Visible absorption spectra were recorded on an Agilent 8453 spectrophotometer. The X-ray single-crystal diffraction was performed on an Agilent SuperNOVA instrument. Melting points (m.p.) were measured on an XT4A apparatus (uncorrected).

Photochemical reactions were performed with a 15 W blue LED [model: GCH-4; $\lambda_{\max }=453 \mathrm{~nm}$ (415-510 nm)] obtained from Wuhan Jiushang Technology Co. LTD. The irradiation vessels are Pyrex glass tube with magnetic stirring bars. The distance from the light source to the irradiation vessel is about $2 \mathrm{~cm}$.

Reactions were monitored by thin layer chromatography (TLC) using pre-coated silica gel plates (GF254). Flash column chromatography was performed on silica gel 60 (particle size 200400 mesh ASTM, purchased from Liangchen, China) and eluted with petroleum ether /ethylacetate. The materials obtained from commercial suppliers were used directly without further purification.

\section{Experimental Procedures}

\section{1) Preparation of substrates}

The symmetrical azobenzenes were synthesized according to the literature procedure.$^{[1]}$ The unsymmetrical azobenzenes were synthesized according to the literature procedure. ${ }^{[2]} \alpha$-Keto acids $\mathbf{2} \mathbf{r}-\mathbf{2 t}$ were obtained from commercial suppliers. The other $\alpha$-keto acids were prepared from oxidation of corresponding methyl ketones by $\mathrm{SeO}_{2}$ according to the reported procedure. ${ }^{[3]}$

\section{2) General procedure for synthesis of 3aa-3oa and 3ab-3at}

Azobenzene 1 (0.2 mmol), $\alpha$-keto acid $\mathbf{2}\left(0.2 \mathrm{mmol}, 1\right.$ equiv) and $\mathrm{CH}_{2} \mathrm{Cl}_{2}(3 \mathrm{~mL})$ were added into a $5 \mathrm{~mL}$ Pyrex glass tube equipped with a magnetic stirring bar. Then, the reaction mixture was stirred under irradiation of a $15 \mathrm{~W}$ blue LED at room temperature. Upon completion of the 
reaction (monitored by TLC), the mixture was concentrated under vacuum and the residue was purified by column chromatography (petrol ether $/ E t O A c=10: 1$ ) to give the pure product 3.

\section{3) General procedure for synthesis of 3pa-3wa}

Azobenzene 1 (0.3 mmol), $\alpha$-keto acid 2 (0.3 mmol, 1 equiv) and $\mathrm{CH}_{2} \mathrm{Cl}_{2}(4.5 \mathrm{~mL})$ were added into a $5 \mathrm{~mL}$ Pyrex glass tube equipped with a magnetic stirring bar. Then, the reaction mixture was stirred under irradiation of a $15 \mathrm{~W}$ blue LED at room temperature for $30 \mathrm{~h}$. Upon completion of the reaction (monitored by TLC), the mixture was concentrated under vacuum and the residue was purified by column chromatography (petrol ether/EtOAc $=15: 1$ ) to give the pure product 3.

\section{4) Procedure for gram-scale synthesis of $3 a a$}

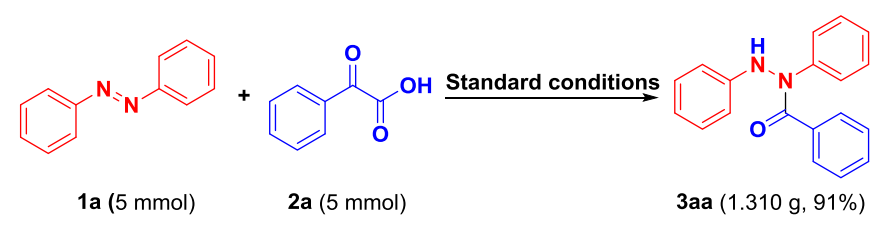

Azobenzenes 1a (0.911 g, $5.0 \mathrm{mmol})$, benzoylformic acid 2a (0.750 g, $5.0 \mathrm{mmol}, 1$ equiv) and $\mathrm{CH}_{2} \mathrm{Cl}_{2}(50.0 \mathrm{~mL})$ were added into a $100 \mathrm{~mL}$ round flask equipped with a magnetic stirring bar. The mixture was then irradiated by two $15 \mathrm{~W}$ blue LEDs and stirred at room temperature for 36 hours. Upon completion (monitored by TLC), the reaction mixture was concentrated under vacuum and the residue was purified by column chromatography (petrol ether/EtOAc $=10: 1$ ) to give the desired product 3aa $(1.31 \mathrm{~g}, 91 \%)$ as a white solid.

\section{5) Crystallographic Structure Determination}

The colorless single crystal of 3ta was obtained by recrystallization using a $\mathrm{CHCl}_{3} /$ Acetone solvent system at room temperature. The X-ray single-crystal diffraction was performed on an Agilent SuperNOVA instrument (Figure S1). 


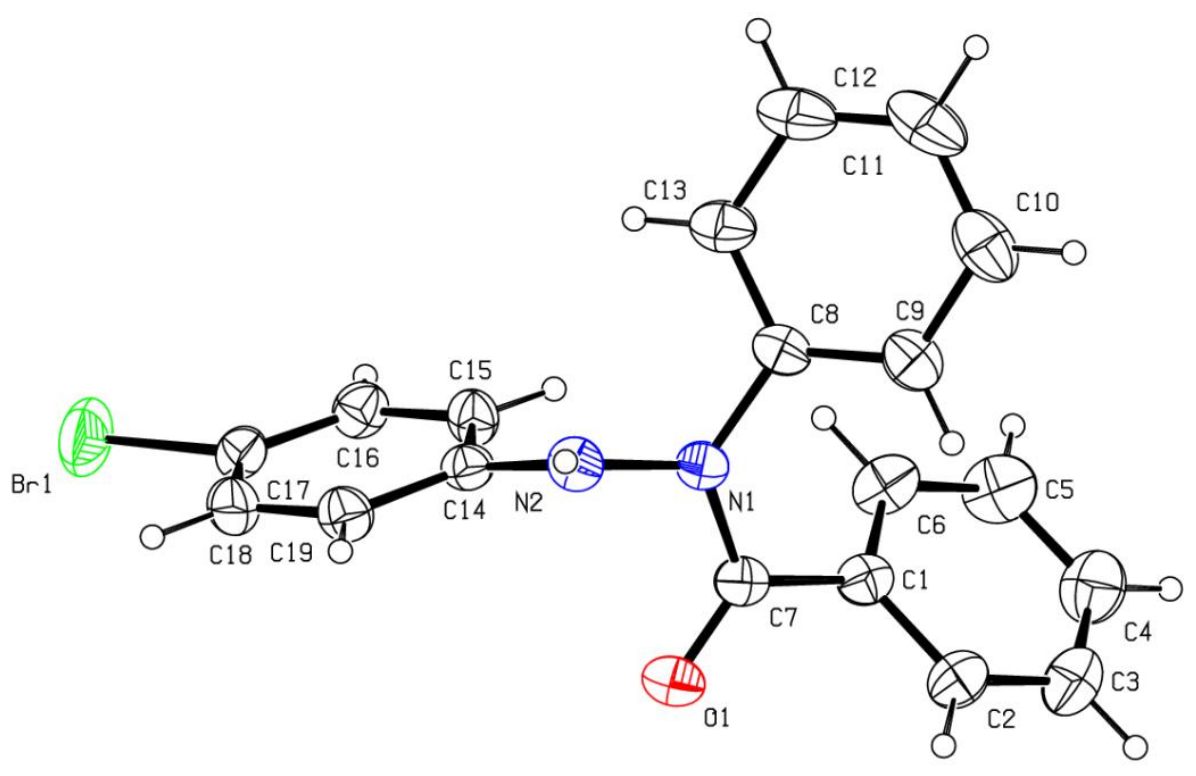

Figure S1. Thermal ellipsoid plot of compound 3ta at the 30\% probability level; hydrogen atoms are drawn as spheres of arbitrary radius.

\section{6) Unsuccessful substrates}

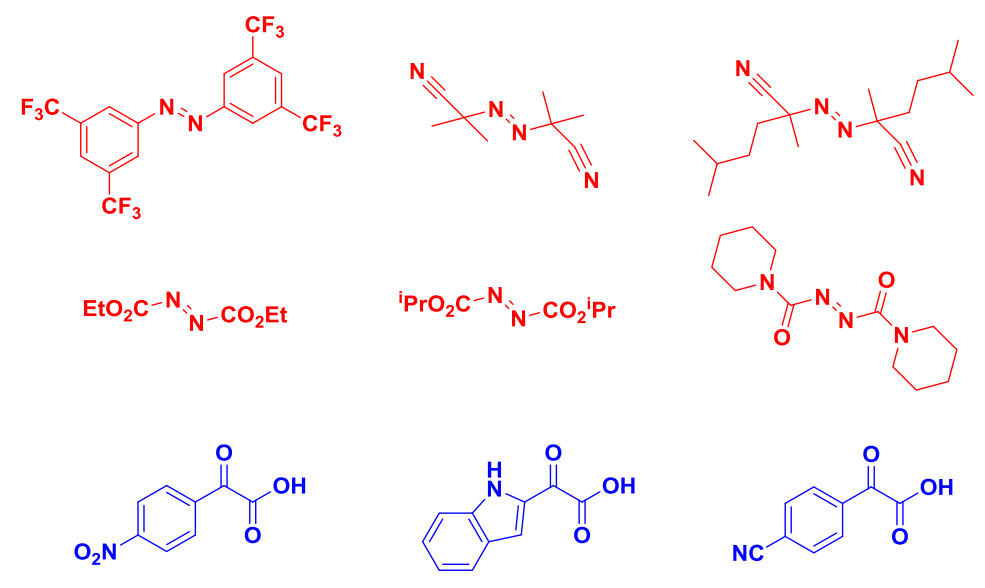

Figure S2. Unsuccessful substrates.

\section{7) Radical-trapping experiment with TEMPO}

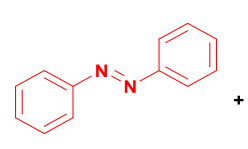

$1 a$

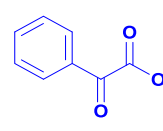

2a

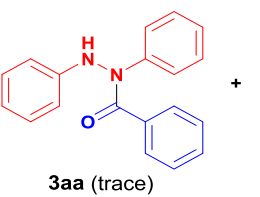

3aa (trace)

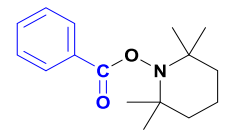

detected by HRMS 
Azobenzene 1a (36.4 mg, $0.2 \mathrm{mmol})$, benzoylformic acid 2a (30.0 mg, $0.2 \mathrm{mmol}, 1$ equiv), TEMPO (93.8 mg, $0.6 \mathrm{mmol}$, 3 equiv) and DCM (3 mL) were added into a $5 \mathrm{~mL}$ Pyrex glass tube. The reaction mixture was continually stirred at room temperature under a $15 \mathrm{~W}$ blue LED irradiation for 24 hours. The reaction solution was concentrated under reduced pressure and the residue was analyzed by TLC and HRMS, respectively. There, only trace amounts of 3aa were detected by TLC and the TEMPO trapped benzoyl radical was detected by HRMS (Figure S3, data of $[\mathrm{M}+\mathrm{H}]^{+}$are showed).

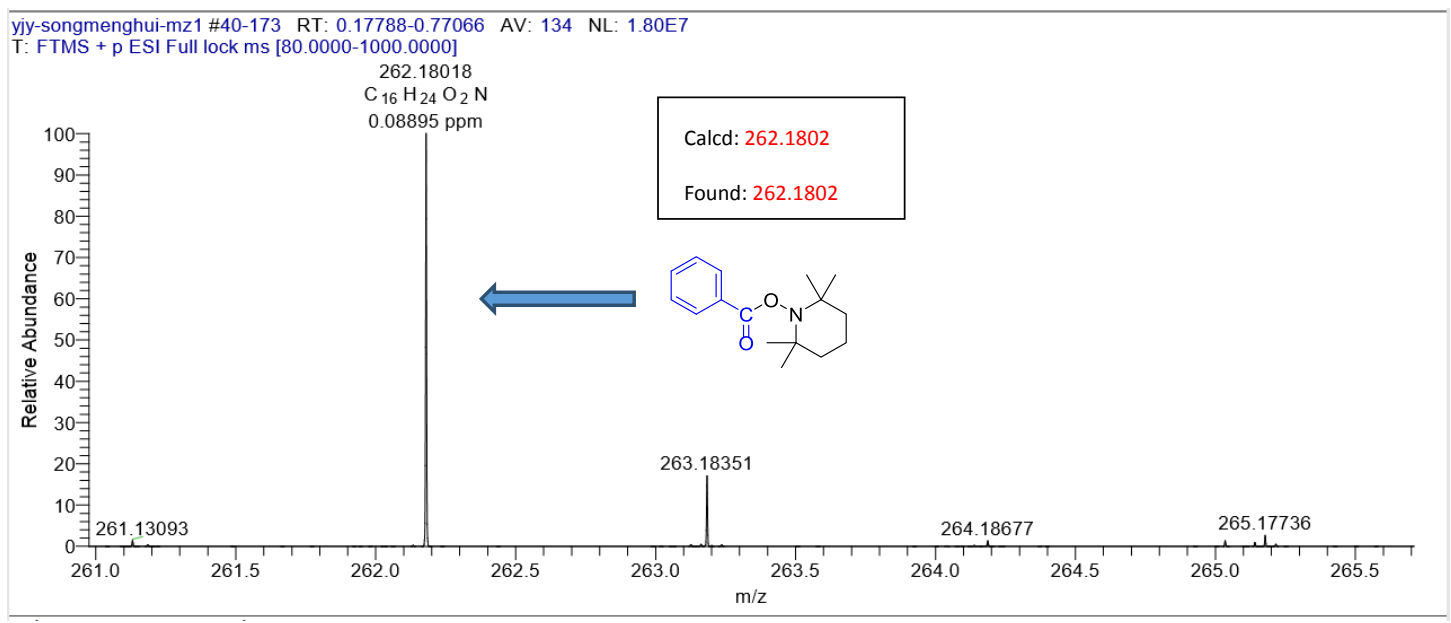

Figure S3

\section{8) Cyclic voltammetry (CV) measurements}

The cyclic voltammetry (CV) measurements were carried out on a $\mathrm{CHI} 660 \mathrm{E}$ electrochemical workstation by a standard three-electrode system (Working electrode: glassy carbon electrode; Counter electrode: Pt wire; Reference electrode: SCE) using tetrabutylammonium perchlorate $(0.025 \mathrm{M})$ as the electrolyte in $\mathrm{DCM}$ at $50 \mathrm{mV} / \mathrm{s}$ scan rate (Figure $\mathbf{S 4}$ ). The excited-state potential of $1 \mathrm{a}\left[E\left(1 \mathrm{a}^{*} / 1 \mathbf{a}^{--}\right)=1.19 \mathrm{~V}\right.$ vs SCE in $\left.\mathrm{CH}_{2} \mathrm{Cl}_{2}\right]$ was calculated using the reduction peak potential of 1a $\left(E_{\text {red }}=-1.06 \mathrm{~V}\right.$ vs SCE in $\left.\mathrm{CH}_{2} \mathrm{Cl}_{2}\right)$ and the excited state energy (2.25 eV) estimated from the wavelength at the tail of absorption of $1 \mathrm{a}\left(\lambda_{\text {tail }}=550 \mathrm{~nm}\right)$ according to the Rehm-Weller equation. 


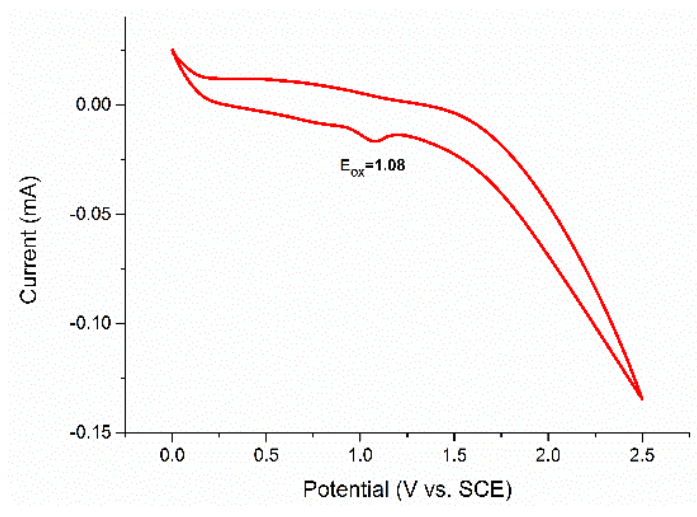

(a)

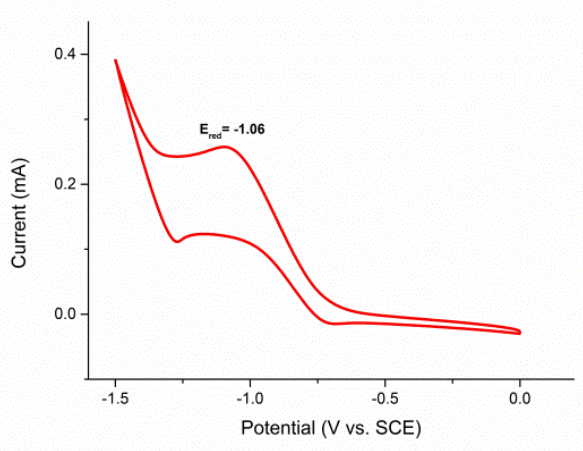

(b)

Figure S4. Cyclic voltammetry (CV) curves of benzoylformic acid $(0.025 \mathrm{M})(\mathrm{a})$ and azobenzene (0.025 M) (b).

\section{9) The on-off-light experiment}

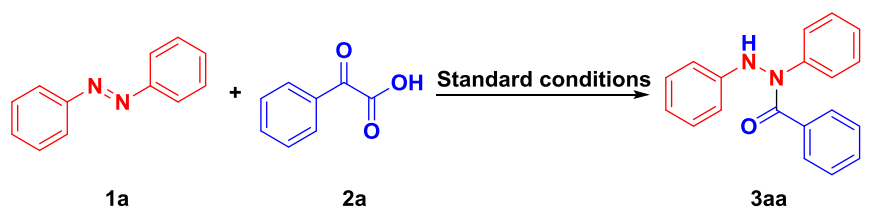

Following the standard procedure, the reaction between azobenzene $1 \mathrm{a}(36.4 \mathrm{mg}, 0.2 \mathrm{mmol}$ ) and benzoylformic acid $2 \mathrm{a}(30.0 \mathrm{mg}, 0.2 \mathrm{mmol}, 1$ equiv) was conducted for on-off-light experiment. Aliquots of samples were taken out at various time points during the reaction. After being irradiated for $2 \mathrm{~h}$, an aliquot was transferred into a nuclear magnetic tube charged and the yield of product 3aa was determined by ${ }^{1} \mathrm{H}$ NMR spectra with $\mathrm{CH}_{2} \mathrm{Br}_{2}$ as internal standard. Then the reaction mixture was stirred for $2 \mathrm{~h}$ with light-off. All of the following yields were analyzed in the identical way after $2 \mathrm{~h}$ light on or off (Figure S5).

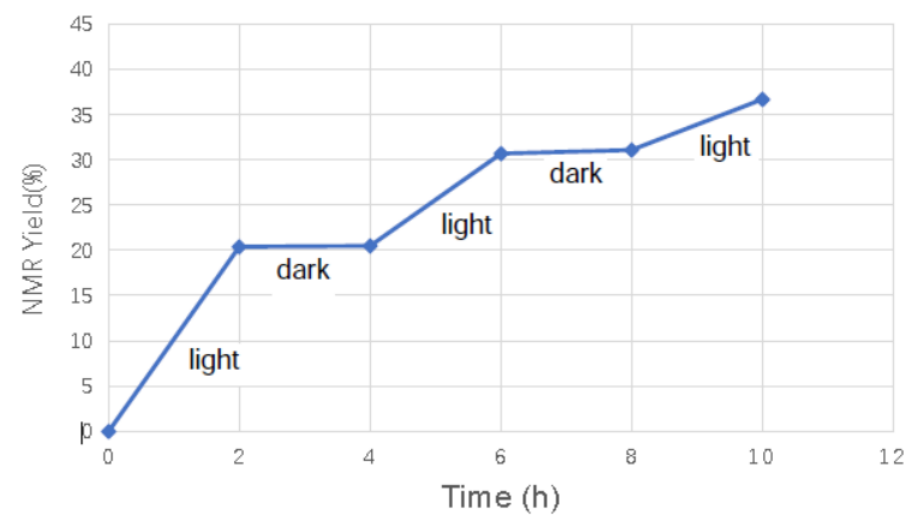

Figure S5. The on/off experiment. 


\section{Characterization Data of Products}

\section{1) $N, N^{\prime}$-Diphenylbenzohydrazide (3aa) $)^{[4]}$}<smiles>O=C(c1ccccc1)N(Nc1ccccc1)c1ccccc1</smiles>

Prepared according to the general procedure from azobenzene 1a (36.4 mg, $0.2 \mathrm{mmol}$ ) and benzoylformic acid $2 \mathrm{a}$ ( $30.0 \mathrm{mg}, 0.2 \mathrm{mmol}, 1$ equiv). Purified by column chromatography on silica gel $(P E: E t O A c=10: 1)$ to afford the pure product 3aa.

White solid; yield: $55.9 \mathrm{mg}(97 \%)$; m.p. $136-138^{\circ} \mathrm{C}$.

${ }^{1} \mathrm{H} \mathrm{NMR}\left(400 \mathrm{MHz}, \mathrm{CDCl}_{3}\right): \delta=7.56(\mathrm{~d}, J=7.6 \mathrm{~Hz}, 2 \mathrm{H}), 7.38(\mathrm{t}, J=7.4 \mathrm{~Hz}, 1 \mathrm{H}), 7.32-7.24(\mathrm{~m}, 8 \mathrm{H}), 7.16(\mathrm{t}$, $J=7.0 \mathrm{~Hz}, 1 \mathrm{H}), 7.09(\mathrm{~s}, 1 \mathrm{H}), 6.96-6.87(\mathrm{~m}, 3 \mathrm{H})$.

${ }^{13} \mathrm{C}$ NMR $\left(150 \mathrm{MHz}, \mathrm{CDCl}_{3}\right): \delta=170.8,147.1,143.1,134.8,130.6,129.2,128.8,128.5,128.0,126.1$, $124.4,121.2,113.4$

HRMS (ESI): $m / z[M+H]^{+}$calcd for $\mathrm{C}_{19} \mathrm{H}_{17} \mathrm{~N}_{2} \mathrm{O}^{+}: 289.1335$; found: 289.1331 .

\section{2) $N, N^{\prime}-$ Di-p-tolylbenzohydrazide (3ba)}<smiles>Cc1ccc(NN(C(=O)c2ccccc2)c2ccc(C)cc2)cc1</smiles>

Prepared according to the general procedure from $(E)-1,2$-di-p-tolyldiazene $\mathbf{1 b}(42.0 \mathrm{mg}, 0.2 \mathrm{mmol})$ and benzoylformic acid $2 \mathrm{a}$ ( $30.0 \mathrm{mg}, 0.2 \mathrm{mmol}, 1$ equiv). Purified by column chromatography on silica gel $(P E:$ EtOAc $=10: 1)$ to afford the pure product $\mathbf{3 b a}$.

White solid; yield: $60.1 \mathrm{mg}(95 \%) ;$ m.p. $125-126^{\circ} \mathrm{C}$.

${ }^{1} \mathrm{H}$ NMR $\left(600 \mathrm{MHz}_{\mathrm{CDCl}}\right): \delta=7.53(\mathrm{~d}, J=7.8 \mathrm{~Hz}, 2 \mathrm{H}), 7.37-7.34(\mathrm{~m}, 1 \mathrm{H}), 7.28-7.25(\mathrm{~m}, 2 \mathrm{H}), 7.15(\mathrm{~d}, J=$ $7.2 \mathrm{~Hz}, 2 \mathrm{H}), 7.04(\mathrm{t}, J=7.5 \mathrm{~Hz}, 4 \mathrm{H}), 6.91(\mathrm{~s}, 1 \mathrm{H}), 6.86(\mathrm{~d}, J=7.8 \mathrm{~Hz}, 2 \mathrm{H}), 2.28(\mathrm{~s}, 3 \mathrm{H}), 2.26(\mathrm{~s}, 3 \mathrm{H})$.

${ }^{13} \mathrm{C}$ NMR $\left(150 \mathrm{MHz}, \mathrm{CDCl}_{3}\right): \delta=170.6,144.7,140.6,136.0,135.0,130.7,130.5,129.8,129.4,128.6$, $127.9,124.6,113.9,20.9,20.5$.

HRMS (ESI): $m / z[M+H]^{+}$calcd for $\mathrm{C}_{21} \mathrm{H}_{21} \mathrm{~N}_{2} \mathrm{O}^{+}: 317.1648$; found: 317.1651 .

\section{3) N,N'-Bis(4-butylphenyl)benzohydrazide (3ca)}


<smiles>O=C(c1ccccc1)N(Nc1ccc(Br)cc1)c1ccc(Br)cc1</smiles>

Prepared according to the general procedure from $(E)$-1,2-bis(4-butylphenyl)diazene 1c (58.9 mg, 0.2 $\mathrm{mmol}$ ) and benzoylformic acid $2 \mathrm{a}(30.0 \mathrm{mg}, 0.2 \mathrm{mmol}, 1$ equiv). Purified by column chromatography on silica gel (PE : EtOAC $=10: 1)$ to afford the pure product $\mathbf{3 c a}$.

White solid; yield: $68.1 \mathrm{mg}(85 \%) ;$ m.p. $69-70^{\circ} \mathrm{C}$.

${ }^{1} \mathrm{H}$ NMR $\left(600 \mathrm{MHz}, \mathrm{CDCl}_{3}\right): \delta=7.52(\mathrm{~d}, J=7.8 \mathrm{~Hz}, 2 \mathrm{H}), 7.34(\mathrm{t}, J=7.5 \mathrm{~Hz}, 1 \mathrm{H}), 7.25(\mathrm{t}, J=7.5 \mathrm{~Hz}, 2 \mathrm{H})$, $7.17(\mathrm{~d}, J=7.8 \mathrm{~Hz}, 2 \mathrm{H}), 7.05(\mathrm{t}, J=7.2 \mathrm{~Hz}, 4 \mathrm{H}), 6.91(\mathrm{~s}, 1 \mathrm{H}), 6.88(\mathrm{~d}, J=7.8 \mathrm{~Hz}, 2 \mathrm{H}), 2.54(\mathrm{q}, J=8.4 \mathrm{~Hz}$ 4H), 1.57-1.53 (m, 4H), 1.36-1.30 (m, 4H), $0.92(q, J=8.4 \mathrm{~Hz}, 6 \mathrm{H})$.

${ }^{13} \mathrm{C}$ NMR $\left(150 \mathrm{MHz}, \mathrm{CDCl}_{3}\right): \delta=170.7,145.1,141.1,141.1,135.9,135.1,130.5,129.1,128.7,128.6$ $127.9,124.6,113.9,35.0,34.8,33.7,33.3,22.3,22.2,13.9,13.8$.

HRMS (ESI): $m / z[M+H]^{+}$calcd for $\mathrm{C}_{27} \mathrm{H}_{33} \mathrm{~N}_{2} \mathrm{O}^{+}: 401.2587$; found: 401.2590 .

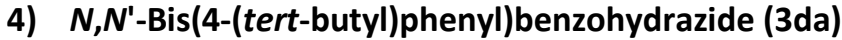<smiles>CC(C)(C)c1ccc(N(Nc2ccc(Br)cc2)C(=O)c2ccccc2)cc1</smiles>

Prepared according to the general procedure from (E)-1,2-bis(4-(tert-butyl)phenyl)diazene 1d (58.9 $\mathrm{mg}, 0.2 \mathrm{mmol}$ ) and benzoylformic acid $2 \mathrm{a}(30.0 \mathrm{mg}, 0.2 \mathrm{mmol}, 1$ equiv). Purified by column chromatography on silica gel $(P E:$ EtOAc $=10: 1)$ to afford the pure product $3 \mathrm{da}$.

White solid; yield: $74.5 \mathrm{mg}(93 \%) ;$ m.p. $166-167^{\circ} \mathrm{C}$.

${ }^{1} \mathrm{H}$ NMR $\left(600 \mathrm{MHz}, \mathrm{CDCl}_{3}\right): \delta=7.54(\mathrm{~d}, J=7.8 \mathrm{~Hz}, 2 \mathrm{H}), 7.37-7.34(\mathrm{~m}, 1 \mathrm{H}), 7.28-7.25(\mathrm{~m}, 6 \mathrm{H}), 7.20(\mathrm{~d}, J$ $=7.2 \mathrm{~Hz}, 2 \mathrm{H}), 6.95(\mathrm{~s}, 1 \mathrm{H}), 6.91(\mathrm{~d}, J=7.8 \mathrm{~Hz}, 2 \mathrm{H}), 1.29(\mathrm{~s}, 9 \mathrm{H}), 1.28(\mathrm{~s}, 9 \mathrm{H})$.

${ }^{13} \mathrm{C}$ NMR $\left(150 \mathrm{MHz}, \mathrm{CDCl}_{3}\right): \delta=170.7,149.2,144.8,144.1,140.7,135.0,130.5,128.7,127.9,126.1$, $125.7,124.2,113.3,34.4,34.1,31.5,31.2$.

HRMS (ESI): $m / z[M+H]^{+}$calcd for $\mathrm{C}_{27} \mathrm{H}_{33} \mathrm{~N}_{2} \mathrm{O}^{+}: 401.2587$; found: 401.2590 .

\section{5) N,N'-Bis(4-fluorophenyl)benzohydrazide (3ea)}


<smiles>O=C(c1ccccc1)N(Nc1ccc(F)cc1)c1ccc(F)cc1</smiles>

Prepared according to the general procedure from (E)-1,2-bis(4-fluorophenyl)diazene 1e (43.6 mg, 0.2 $\mathrm{mmol}$ ) and benzoylformic acid $2 \mathrm{a}(30.0 \mathrm{mg}, 0.2 \mathrm{mmol}, 1$ equiv). Purified by column chromatography on silica gel (PE : EtOAc $=10: 1)$ to afford the pure product 3 ea.

White solid; yield: $60.3 \mathrm{mg}(93 \%) ;$ m.p. $116-118^{\circ} \mathrm{C}$.

${ }^{1} \mathrm{H}$ NMR $\left(600 \mathrm{MHz}, \mathrm{CDCl}_{3}\right): \delta=7.48(\mathrm{~d}, J=7.2 \mathrm{~Hz}, 2 \mathrm{H}), 7.40-7.37(\mathrm{~m}, 1 \mathrm{H}), 7.28(\mathrm{t}, J=7.8 \mathrm{~Hz}, 2 \mathrm{H}), 7.21$ (s, 2H), $7.06(\mathrm{~s}, 1 \mathrm{H}), 6.95-6.92(\mathrm{~m}, 4 \mathrm{H}), 6.87(\mathrm{~s}, 2 \mathrm{H})$.

${ }^{13} \mathrm{C}$ NMR $\left(150 \mathrm{MHz}, \mathrm{CDCl}_{3}\right): \delta=170.6,161.4(\mathrm{~d}, J=224.5 \mathrm{~Hz}), 158.8(\mathrm{~d}, J=237.0 \mathrm{~Hz}), 142.9,139.0$, $134.4,130.9,128.5,128.1,126.4,116.0(d, J=43.5 \mathrm{~Hz}), 115.83,115.2$

${ }^{19} \mathrm{~F} \mathrm{NMR}\left(287 \mathrm{MHz}, \mathrm{CDCl}_{3}\right): \delta=-120.04,-127.76$.

HRMS (ESI): $m / z[M+H]^{+}$calcd for $\mathrm{C}_{19} \mathrm{H}_{15} \mathrm{~F}_{2} \mathrm{~N}_{2} \mathrm{O}^{+}$: 325.1147; found: 325.1151 .

\section{6) $N, N^{\prime}$-Bis(4-chlorophenyl)benzohydrazide (3fa)}<smiles>O=C(c1ccccc1)N(Nc1ccc(Cl)cc1)c1ccc(Cl)cc1</smiles>

Prepared according to the general procedure from (E)-1,2-bis(4-chlorophenyl)diazene $1 \mathbf{1 f}(50.2 \mathrm{mg}, 0.2$ $\mathrm{mmol}$ ) and benzoylformic acid $2 \mathrm{a}(30.0 \mathrm{mg}, 0.2 \mathrm{mmol}, 1$ equiv). Purified by column chromatography on silica gel (PE : EtOAc $=10: 1)$ to afford the pure product $\mathbf{3 f a}$.

White solid; yield: $65.7 \mathrm{mg}(92 \%) ;$ m.p. $146-147^{\circ} \mathrm{C}$.

${ }^{1} \mathrm{H}$ NMR $\left(600 \mathrm{MHz}, \mathrm{CDCl}_{3}\right): \delta=7.49-7.46(\mathrm{~m}, 2 \mathrm{H}), 7.42-7.39(\mathrm{~m}, 1 \mathrm{H}), 7.29(\mathrm{t}, J=7.8 \mathrm{~Hz}, 2 \mathrm{H}), 7.23-7.15$ (m, 6H), 7.05-7.01 (m, 1H), $6.80(\mathrm{~d}, J=4.8 \mathrm{~Hz}, 2 \mathrm{H})$.

${ }^{13} \mathrm{C}$ NMR $\left(150 \mathrm{MHz}, \mathrm{CDCl}_{3}\right): \delta=170.7,145.3,141.3,134.2,131.7,131.1,129.3,129.0,128.4,128.2$, $126.1,125.4,114.6$

HRMS (ESI): $m / z[M+H]^{+}$calcd for $\mathrm{C}_{19} \mathrm{H}_{15} \mathrm{Cl}_{2} \mathrm{~N}_{2} \mathrm{O}^{+}$: 357.0556; found: 357.0561 .

\section{7) N,N'-Bis(4-bromophenyl)benzohydrazide (3ga)}


<smiles>O=C(c1ccccc1)N(Nc1ccc(Br)cc1)c1ccc(Br)cc1</smiles>

Prepared according to the general procedure from (E)-1,2-bis(4-bromophenyl)diazene $1 \mathrm{~g}$ (68.0 $\mathrm{mg}$, $0.2 \mathrm{mmol}$ ) and benzoylformic acid $2 \mathrm{a}(30.0 \mathrm{mg}, 0.2 \mathrm{mmol}, 1$ equiv). Purified by column chromatography on silica gel $(\mathrm{PE}:$ EtOAc $=10: 1)$ to afford the pure product 3ga.

White solid; yield: $82.1 \mathrm{mg}$ (92\%); m.p. $159-160^{\circ} \mathrm{C}$.

${ }^{1} \mathrm{H}$ NMR $\left(400 \mathrm{MHz}, \mathrm{CDCl}_{3}\right): \delta=7.46-7.44(\mathrm{~m}, 2 \mathrm{H}), 7.42-7.18(\mathrm{~m}, 8 \mathrm{H}), 7.11(\mathrm{~d}, J=6.4 \mathrm{~Hz}, 2 \mathrm{H}), 6.67(\mathrm{~d}, J$

$=7.6 \mathrm{~Hz}, 2 \mathrm{H})$.

${ }^{13} \mathrm{C} \mathrm{NMR}\left(150 \mathrm{MHz}, \mathrm{CDCl}_{3}\right): \delta=170.7,145.7,141.7,134.2,132.1,131.9,131.1,128.3,128.2,125.7$,

$119.5,114.9,113.3$

HRMS (ESI): $\mathrm{m} / \mathrm{z}[\mathrm{M}+\mathrm{H}]^{+}$calcd for $\mathrm{C}_{19} \mathrm{H}_{15} \mathrm{Br}_{2} \mathrm{~N}_{2} \mathrm{O}^{+}$: 446.9525; found: 446.9528 .

\section{8) N,N'-Bis(4-(trifluoromethyl)phenyl)benzohydrazide (3ha)}<smiles>O=C(c1ccccc1)N(Nc1ccc(C(F)(F)F)cc1)c1ccc(C(F)(F)F)cc1</smiles>

Prepared according to the general procedurefrom (E)-1,2-bis(4-(trifluoromethyl)phenyl)diazene $\mathbf{1} \mathbf{h}$ (63.6 $\mathrm{mg}, 0.2 \mathrm{mmol}$ ) and benzoylformic acid $2 \mathrm{a}(30.0 \mathrm{mg}, 0.2 \mathrm{mmol}, 1$ equiv). Purified by column chromatography on silica gel (PE : EtOAC $=10: 1)$ to afford the pure product 3ha.

White solid; yield: $56.8 \mathrm{mg}$ (67\%); m.p. $132-133^{\circ} \mathrm{C}$.

${ }^{1} \mathrm{H}$ NMR $\left(600 \mathrm{MHz}, \mathrm{CDCl}_{3}\right): \delta=7.52-7.47(\mathrm{~m}, 6 \mathrm{H}), 7.45(\mathrm{t}, J=7.2 \mathrm{~Hz}, 1 \mathrm{H}), 7.37(\mathrm{~d}, J=8.4 \mathrm{~Hz}, 2 \mathrm{H}), 7.32(\mathrm{t}$, $J=7.8 \mathrm{~Hz}, 3 \mathrm{H}), 6.88(\mathrm{~d}, J=6.6 \mathrm{~Hz}, 2 \mathrm{H})$.

${ }^{13} \mathrm{CNMR}\left(150 \mathrm{MHz}, \mathrm{CDCl}_{3}\right): \delta=170.9,149.4,145.7,133.9,131.6,128.5,128.4,128.2$ (d, $\left.J=31.5 \mathrm{~Hz}\right)$, $126.9(\mathrm{q}, J=3.0 \mathrm{~Hz}), 126.2(\mathrm{q}, J=3.0 \mathrm{~Hz}), 125.2(\mathrm{~d}, J=88.5 \mathrm{~Hz}), 123.7,123.4,122.8,112.6$.

${ }^{19} \mathrm{~F} \mathrm{NMR}\left(287 \mathrm{MHz}, \mathrm{CDCl}_{3}\right): \delta=-66.86,-67.59$.

HRMS (ESI): $m / z[\mathrm{M}+\mathrm{Na}]^{+}$calcd for $\mathrm{C}_{21} \mathrm{H}_{15} \mathrm{~F}_{6} \mathrm{~N}_{2} \mathrm{ONa}^{+}: 447.0903$; found: 447.0906 .

\section{9) N,N'-Bis(4-(trifluoromethoxy)phenyl)benzohydrazide (3ia)}<smiles>CCOc1ccc(N(C(=O)c2ccccc2)c2ccc(OC(F)(F)F)cc2)cc1</smiles> 
Prepared according to the general procedure from (E)-1,2-bis(4-(trifluoromethoxy)phenyl)diazene $\mathbf{1} \mathbf{i}$ (70.0 mg, $0.2 \mathrm{mmol}$ ) and benzoylformic acid $2 \mathrm{a}(30.0 \mathrm{mg}, 0.2 \mathrm{mmol}, 1$ equiv). Purified by column chromatography on silica gel (PE : EtOAc $=10: 1)$ to afford the pure product 3ia.

White solid; yield: $81.2 \mathrm{mg}(89 \%) ;$ m.p. $76-78^{\circ} \mathrm{C}$.

${ }^{1} \mathrm{H}$ NMR $\left(600 \mathrm{MHz}^{\mathrm{C} C D C l}{ }_{3}\right): \delta=7.48-7.46(\mathrm{~m}, 2 \mathrm{H}), 7.42-7.39(\mathrm{~m}, 1 \mathrm{H}), 7.30-7.25(\mathrm{~m}, 4 \mathrm{H}), 7.10(\mathrm{~d}, J=9.0$ $\mathrm{Hz}, 5 \mathrm{H}), 6.87(\mathrm{~d}, J=8.4 \mathrm{~Hz}, 2 \mathrm{H})$.

${ }^{13} \mathrm{CNMR}\left(150 \mathrm{MHz}, \mathrm{CDCl}_{3}\right): \delta=170.7,146.9(\mathrm{~d}, J=204.0 \mathrm{~Hz}), 143.5,141.5,134.1,131.3,128.5,128.3$, $125.5,123.1,122.5$ (d, J =163.5 Hz), 121.2, 119.7, 119.5, 114.2.

${ }^{19} \mathrm{~F} \mathrm{NMR}\left(287 \mathrm{MHz}, \mathrm{CDCl}_{3}\right): \delta=-58.47,-58.76$.

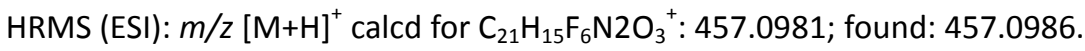

\section{0) $N, N^{\prime}-$ Di-m-tolylbenzohydrazide (3ja)}<smiles>Cc1cccc(NN(C(=O)c2ccccc2)c2cccc(C)c2)c1</smiles>

Prepared according to the general procedure from $(E)$-1,2-di-m-tolyldiazene $1 \mathbf{j}(42.0 \mathrm{mg}, 0.2 \mathrm{mmol})$ and benzoylformic acid 2 a ( $30.0 \mathrm{mg}, 0.2 \mathrm{mmol}, 1$ equiv). Purified by column chromatography on silica gel $(P E:$ EtOAC $=10: 1)$ to afford the pure product $\mathbf{3 j a}$.

White solid; yield: $58.2 \mathrm{mg}(92 \%) ;$ m.p. $102-103^{\circ} \mathrm{C}$.

${ }^{1} \mathrm{H}$ NMR $\left(600 \mathrm{MHz}_{\mathrm{CDCl}}\right): \delta=7.54-7.51(\mathrm{~m}, 2 \mathrm{H}), 7.37-7.34(\mathrm{~m}, 1 \mathrm{H}), 7.28-7.26(\mathrm{~m}, 2 \mathrm{H}), 7.14-7.08(\mathrm{~m}$, 3H), $6.98(\mathrm{~d}, J=7.8 \mathrm{~Hz}, 1 \mathrm{H}), 6.95(\mathrm{~d}, J=7.2 \mathrm{~Hz}, 1 \mathrm{H}), 6.86(\mathrm{~s}, 1 \mathrm{H}), 6.78-6.72(\mathrm{~m}, 3 \mathrm{H}), 2.29(\mathrm{~s}, 3 \mathrm{H}), 2.26(\mathrm{~s}$, $3 \mathrm{H})$.

${ }^{13} \mathrm{CNMR}\left(150 \mathrm{MHz}, \mathrm{CDCl}_{3}\right): \delta=170.8,147.3,143.3,139.2,138.9,134.9,130.7,129.2,128.7,128.5$, $128.0,127.1,125.0,122.4,121.9,114.4,110.6,21.6,21.3$.

HRMS (ESI): $m / z[M+H]^{+}$calcd for $\mathrm{C}_{21} \mathrm{H}_{21} \mathrm{~N}_{2} \mathrm{O}^{+}: 317.1648$; found: 317.1652 .

\section{1) $N, N^{\prime}-$ Bis(3-fluorophenyl)benzohydrazide (3ka)}<smiles>O=C(c1ccccc1)N(Nc1cccc(F)c1)c1cccc(F)c1</smiles>

Prepared according to the general procedure from (E)-1,2-bis(3-fluorophenyl)diazene $\mathbf{1 k}$ (43.6 mg, 0.2 
mmol) and benzoylformic acid $2 a(30.0 \mathrm{mg}, 0.2 \mathrm{mmol}, 1$ equiv). Purified by column chromatography on silica gel (PE : EtOAc $=10: 1)$ to afford the pure product 3ka.

White solid; yield: $56.4 \mathrm{mg}(87 \%)$; m.p. $122-123^{\circ} \mathrm{C}$.

${ }^{1} \mathrm{H} \mathrm{NMR}\left(400 \mathrm{MHz}, \mathrm{CDCl}_{3}\right): \delta=7.51-7.50(\mathrm{~m}, 2 \mathrm{H}), 7.41(\mathrm{t}, J=7.5 \mathrm{~Hz}, 1 \mathrm{H}), 7.30(\mathrm{t}, J=7.8 \mathrm{~Hz}, 2 \mathrm{H}), 7.21-$ $7.12(m, 3 H), 7.06(d, J=10.2 \mathrm{~Hz}, 1 \mathrm{H}), 7.02(\mathrm{~d}, J=8.4 \mathrm{~Hz}, 1 \mathrm{H}), 6.88-6.85(\mathrm{~m}, 1 \mathrm{H}), 6.64-6.56(\mathrm{~m}, 3 \mathrm{H})$.

${ }^{13} \mathrm{C} \mathrm{NMR}\left(150 \mathrm{MHz}, \mathrm{CDCl}_{3}\right): \delta=170.8,164.6(\mathrm{~d}, J=168.0 \mathrm{~Hz}), 163.0(\mathrm{~d}, J=171.0 \mathrm{~Hz}), 148.8(\mathrm{~d}, J=9.0$

$\mathrm{Hz}), 144.4(\mathrm{~d}, J=9.0 \mathrm{~Hz}), 134.2,131.2,130.8(\mathrm{~d}, J=9 \mathrm{~Hz}), 130.0(\mathrm{~d}, J=9.0 \mathrm{~Hz}), 128.4,128.2,119.8$,

$113.28(\mathrm{~d}, J=21 \mathrm{~Hz}), 111.5(\mathrm{~d}, J=24.0 \mathrm{~Hz}), 108.9,108.2(\mathrm{~d}, J=21.0 \mathrm{~Hz}), 100.70(\mathrm{~d}, J=25.5 \mathrm{~Hz})$.

${ }^{19} \mathrm{~F} \mathrm{NMR}\left(376 \mathrm{MHz}, \mathrm{CDCl}_{3}\right): \delta=-116.21,-116.77$.

HRMS (ESI): $m / z[M+H]^{+}$calcd for $\mathrm{C}_{19} \mathrm{H}_{15} \mathrm{~F}_{2} \mathrm{~N}_{2} \mathrm{O}^{+}$: 325.1147; found: 325.1149 .

\section{2) $N, N$ '-Bis(3-chlorophenyl)benzohydrazide (3la)}<smiles>O=C(c1ccccc1)N(Nc1cccc(Cl)c1)c1cccc(Cl)c1</smiles>

Prepared according to the general procedure from (E)-1,2-bis(3-chlorophenyl)diazene 1l (50.2 mg, 0.2 $\mathrm{mmol}$ ) and benzoylformic acid $2 \mathrm{a}(30.0 \mathrm{mg}, 0.2 \mathrm{mmol}, 1$ equiv). Purified by column chromatography on silica gel (PE: EtOAc $=10: 1)$ to afford the pure product 3la.

White solid; yield: $61.4 \mathrm{mg}(86 \%) ;$ m.p. $112-113^{\circ} \mathrm{C}$.

${ }^{1} \mathrm{H}$ NMR $\left(600 \mathrm{MHz}, \mathrm{CDCl}_{3}\right): \delta=7.50-7.48(\mathrm{~m}, 2 \mathrm{H}), 7.42-7.39(\mathrm{~m}, 1 \mathrm{H}), 7.34(\mathrm{~s}, 1 \mathrm{H}), 7.31-7.28(\mathrm{~m}, 2 \mathrm{H})$, 7.16-7.11 (m, 4H), $7.08(\mathrm{~d}, J=6.6 \mathrm{~Hz}, 1 \mathrm{H}), 6.89-6.87(\mathrm{~m}, 1 \mathrm{H}), 6.84(\mathrm{~s}, 1 \mathrm{H}), 6.74-6.73(\mathrm{~m}, 1 \mathrm{H})$.

${ }^{13} \mathrm{C}$ NMR $\left(150 \mathrm{MHz}, \mathrm{CDCl}_{3}\right): \delta=170.7,148.1,144.1,135.2,134.6,134.1,131.3,130.5,129.7,128.5$, $128.3,126.5,124.1,122.5,121.6,113.4,111.5$.

HRMS (ESI): $m / z[M+H]^{+}$calcd for $\mathrm{C}_{19} \mathrm{H}_{15} \mathrm{Cl}_{2} \mathrm{~N}_{2} \mathrm{O}^{+}$: 357.0556; found: 357.0559 .

\section{3) $N, N^{\prime}$-Bis(3-bromophenyl)benzohydrazide (3ma)}<smiles>O=C(c1ccccc1)N(Nc1cccc(Br)c1)c1cccc(Br)c1</smiles>

Prepared according to the general procedure from (E)-1,2-bis(3-bromophenyl)diazene $1 \mathrm{~m}(68.0 \mathrm{mg}$, $0.2 \mathrm{mmol}$ ) and benzoylformic acid $2 \mathrm{a}(30.0 \mathrm{mg}, 0.2 \mathrm{mmol}, 1$ equiv). Purified by column 
chromatography on silica gel (PE: EtOAc $=10: 1)$ to afford the pure product 3ma.

White solid; yield: $62.4 \mathrm{mg}(70 \%) ;$ m.p. $165-167^{\circ} \mathrm{C}$.

${ }^{1} \mathrm{H}$ NMR $\left(600 \mathrm{MHz}, \mathrm{CDCl}_{3}\right): \delta=7.49(\mathrm{~d}, J=7.8 \mathrm{~Hz}, 3 \mathrm{H}), 7.42(\mathrm{t}, J=7.5 \mathrm{~Hz}, 1 \mathrm{H}), 7.33-7.27(\mathrm{~m}, 3 \mathrm{H}), 7.11-$ $7.07(\mathrm{~m}, 3 \mathrm{H}), 7.40(\mathrm{~d}, J=7.5 \mathrm{~Hz}, 2 \mathrm{H}), 7.00(\mathrm{~s}, 1 \mathrm{H}), 6.79(\mathrm{~d}, J=6.6 \mathrm{~Hz}, 1 \mathrm{H})$.

${ }^{13} \mathrm{C}$ NMR $\left(150 \mathrm{MHz}, \mathrm{CDCl}_{3}\right): \delta=170.7,148.1,144.1,134.0,131.3,130.8,130.0,129.4,128.5,128.3$, $126.9,124.5,123.3,122.9,122.5,116.3,111.9$.

HRMS (ESI): $m / z[M+H]^{+}$calcd for $\mathrm{C}_{19} \mathrm{H}_{15} \mathrm{Br}_{2} \mathrm{~N}_{2} \mathrm{O}^{+}: 446.9525$; found: 446.9528 .

\section{4) N,N'-Di-o-tolylbenzohydrazide (3na)}<smiles>Cc1ccccc1NN(C(=O)c1ccccc1)c1ccccc1C</smiles>

Prepared according to the general procedure from $(E)$-1,2-di-o-tolyldiazene $1 \mathrm{n}(42.0 \mathrm{mg}, 0.2 \mathrm{mmol})$ and benzoylformic acid $2 \mathrm{a}$ ( $30.0 \mathrm{mg}, 0.2 \mathrm{mmol}, 1$ equiv). Purified by column chromatography on silica gel $(\mathrm{PE}: \mathrm{EtOAC}=10: 1)$ to afford the pure product 3 na.

White solid; yield: $56.3 \mathrm{mg}(89 \%) ;$ m.p. $121-122^{\circ} \mathrm{C}$.

${ }^{1} \mathrm{H}$ NMR $\left(600 \mathrm{MHz}, \mathrm{CDCl}_{3}\right): \delta=7.49(\mathrm{~s}, 1 \mathrm{H}), 7.33-7.15(\mathrm{~m}, 11 \mathrm{H}), 6.95-6.89(\mathrm{~m}, 2 \mathrm{H}), 2.78(\mathrm{~s}, 3 \mathrm{H}), 2.33(\mathrm{~s}$, $3 \mathrm{H})$.

${ }^{13} \mathrm{C}$ NMR $\left(150 \mathrm{MHz}, \mathrm{CDCl}_{3}\right): \delta=170.9,144.6,144.6,142.1,134.8,134.6,131.4,130.6,130.5,128.5$, $127.8,127.75,127.7,126.7,126.5,121.2,113.1,18.5,16.9$.

HRMS (ESI): $m / z[M+H]^{+}$calcd for $\mathrm{C}_{21} \mathrm{H}_{21} \mathrm{~N}_{2} \mathrm{O}^{+}: 317.1648$; found: 317.1651 .

15) N,N'-Bis(3,4-dimethylphenyl)benzohydrazide (3oa)<smiles>Cc1ccc(NN(C(=O)c2ccccc2)c2ccc(C)c(C)c2)cc1C</smiles>

Prepared according to the general procedure from $(E)$-1,2-Bis(3,4-dimethylphenyl)diazene 10 (47.7 $\mathrm{mg}, 0.2 \mathrm{mmol}$ ) and benzoylformic acid $2 \mathrm{a}(30.0 \mathrm{mg}, 0.2 \mathrm{mmol}, 1$ equiv). Purified by column chromatography on silica gel $(\mathrm{PE}:$ EtOAc $=10: 1)$ to afford the pure product 3oa.

White solid; yield: $62.7 \mathrm{mg}(91 \%) ;$ m.p. $166-167^{\circ} \mathrm{C}$.

${ }^{1} \mathrm{H}$ NMR $\left(400 \mathrm{MHz}, \mathrm{CDCl}_{3}\right): \delta=7.57(\mathrm{t}, J=4.2 \mathrm{~Hz}, 2 \mathrm{H}), 7.38-7.34(\mathrm{~m}, 1 \mathrm{H}), 7.30-7.25(\mathrm{~m}, 2 \mathrm{H}), 7.14(\mathrm{~s}$, 
$1 \mathrm{H}), 7.02-6.87(\mathrm{~m}, 4 \mathrm{H}), 6.76(\mathrm{~d}, J=8.0 \mathrm{~Hz}, 2 \mathrm{H}), 2.22-2.18(\mathrm{~m}, 12 \mathrm{H})$.

${ }^{13} \mathrm{C}$ NMR $\left(150 \mathrm{MHz}, \mathrm{CDCl}_{3}\right): \delta=170.8,145.2,141.0,137.4,137.3,135.2,134.8,130.5,130.3,129.8$, $129.4,128.7,127.9,125.7,122.3,115.5,111.2,20.1,19.9,19.3,18.9$.

HRMS (ESI): $m / z[M+H]^{+}$calcd for $\mathrm{C}_{23} \mathrm{H}_{25} \mathrm{~N}_{2} \mathrm{O}^{+}: 345.1961$; found: 345.1964 .

\section{6) $3 p a+3 p a '$}<smiles>Cc1ccc(NN(C(=O)c2ccccc2)c2ccccc2)cc1</smiles>

Prepared according to the general procedure from $(E)-1$-phenyl-2-(p-tolyl)diazene $1 \mathrm{p}(58.9 \mathrm{mg}, 0.3$ mmol) and benzoylformic acid $2 a$ ( $45.0 \mathrm{mg}, 0.3 \mathrm{mmol}, 1$ equiv). Purified by column chromatography on silica gel (PE: EtOAc $=15: 1)$ to afford the mixture products 3pa+3pa'.

White solid; yield: $85.3 \mathrm{mg}(94 \%)$.

${ }^{1} \mathrm{H}$ NMR $\left(400 \mathrm{MHz}, \mathrm{CDCl}_{3}\right): \delta=7.56(\mathrm{~d}, J=7.6 \mathrm{~Hz}, 4 \mathrm{H}), 7.40-7.35(\mathrm{~m}, 2 \mathrm{H}), 7.33-7.11(\mathrm{~m}, 15 \mathrm{H}), 7.06(\mathrm{~d}, J$ $=7.6 \mathrm{~Hz}, 4 \mathrm{H}), 6.93(\mathrm{~m}, 3 \mathrm{H}), 6.86(\mathrm{~d}, J=7.6 \mathrm{~Hz}, 2 \mathrm{H}), 2.30(\mathrm{~s}, 3 \mathrm{H}), 2.29(\mathrm{~s}, 3 \mathrm{H})$.

${ }^{13} \mathrm{C}$ NMR $\left(150 \mathrm{MHz}, \mathrm{CDCl}_{3}\right): \delta=170.7,147.2,144.7,143.2,140.7,136.0,135.1,130.6,130.5,130.4$, 129.7, 129.4, 129.1, 128.7, 128.5, 127.86, 127.85, 126.0, 124.5, 124.4, 121.1, 113.9, 113.6, 20.8, 20.4. HRMS (ESI): $m / z[M+H]^{+}$calcd for $\mathrm{C}_{20} \mathrm{H}_{19} \mathrm{~N}_{2} \mathrm{O}^{+}:$303.1492; found: 303.1495 .

17) $3 q a+3 q a '$<smiles>CC(C)(C)c1ccc(NN(C(=O)c2ccccc2)c2ccccc2)cc1</smiles>

$3 q a$<smiles>CC(C)(C)c1ccc(N(Nc2ccccc2)C(=O)c2ccccc2)cc1</smiles>

3qa'

Prepared according to the general procedure from (E)-1-(4-(tert-butyl)phenyl)-2-phenyldiazene $\mathbf{1 q}$ (71.5 mg, $0.3 \mathrm{mmol}$ ) and benzoylformic acid $2 \mathrm{a}(45.0 \mathrm{mg}, 0.3 \mathrm{mmol}, 1$ equiv). Purified by column chromatography on silica gel (PE: EtOAc $=15: 1)$ to afford the mixture products 3qa+3qa'.

White solid; yield: $95.1 \mathrm{mg}(92 \%)$.

${ }^{1} \mathrm{H}$ NMR $\left(600 \mathrm{MHz}, \mathrm{CDCl}_{3}\right): \delta=7.56(\mathrm{~m}, 4 \mathrm{H}), 7.39-7.35(\mathrm{~m}, 2 \mathrm{H}), 7.26(\mathrm{~m}, 17 \mathrm{H}), 7.14(\mathrm{t}, J=7.5 \mathrm{~Hz}, 1 \mathrm{H})$, $7.08(\mathrm{~s}, 1 \mathrm{H}), 6.92(\mathrm{~m}, 5 \mathrm{H}), 1.31(\mathrm{~s}, 9 \mathrm{H}), 1.30(\mathrm{~s}, 9 \mathrm{H})$.

${ }^{13} \mathrm{C}$ NMR $\left(150 \mathrm{MHz}, \mathrm{CDCl}_{3}\right): \delta=170.9,170.6,149.1,147.0,144.6,143.9,143.3,140.3,135.0,134.8$, 
130.6, 130.5, 129.2, 128.7 128.6, 128.4, 127.9, 127.8, 126.0, 126.0, 125.6, 124.6, 123.8, 121.0, 113.3, $113.2,34.3,34.0,31.4,31.2$.

HRMS (ESI): $\mathrm{m} / \mathrm{z}[\mathrm{M}+\mathrm{H}]^{+}$calcd for $\mathrm{C}_{23} \mathrm{H}_{25} \mathrm{~N}_{2} \mathrm{O}^{+}: 345.1961$; found: 345.1956 .

18) $N^{\prime}-(4-M e t h o x y p h e n y l)-N$-phenylbenzohydrazide (3ra)<smiles>COc1ccc(NN(C(=O)c2ccccc2)c2ccccc2)cc1</smiles>

Prepared according to the general procedure from (E)-1-(4-methoxyphenyl)-2-phenyldiazene $1 \mathrm{r}$ (63.7 $\mathrm{mg}, 0.3 \mathrm{mmol}$ ) and benzoylformic acid $2 \mathrm{a}(45.0 \mathrm{mg}, 0.3 \mathrm{mmol}, 1$ equiv). Purified by column chromatography on silica gel (PE: EtOAc $=15: 1)$ to afford the pure product 3ra.

White solid; yield: $45.8 \mathrm{mg}(48 \%)$; m.p. $149-151^{\circ} \mathrm{C}$.

${ }^{1} \mathrm{H}$ NMR $\left(400 \mathrm{MHz}_{\mathrm{CDCl}}\right): \delta=7.53(\mathrm{~d}, J=7.6 \mathrm{~Hz}, 2 \mathrm{H}), 7.36-7.33(\mathrm{~m}, 1 \mathrm{H}), 7.28-7.18(\mathrm{~m}, 6 \mathrm{H}), 7.01-6.89$ $(\mathrm{m}, 4 \mathrm{H}), 6.76(\mathrm{~d}, J=9.2 \mathrm{~Hz}, 2 \mathrm{H}), 3.73(\mathrm{~s}, 3 \mathrm{H})$.

${ }^{13} \mathrm{C}$ NMR $\left(150 \mathrm{MHz}, \mathrm{CDCl}_{3}\right): \delta=170.7,157.7,147.0,134.9,130.4,129.1,128.5,127.8,126.1,121.1$, $114.0,113.5,55.2$.

HRMS (ESI): $m / z[M+H]^{+}$calcd for $\mathrm{C}_{20} \mathrm{H}_{19} \mathrm{~N}_{2} \mathrm{O}_{2}{ }^{+}: 319.1441$; found: 319.1445 .

\section{9) $N$-(4-Methoxyphenyl)-N'-phenylbenzohydrazide (3ra')}<smiles>COc1ccc(N(Nc2ccccc2)C(=O)c2ccccc2)cc1</smiles>

Prepared according to the general procedure from (E)-1-(4-methoxyphenyl)-2-phenyldiazene $1 \mathrm{r}$ (63.7 $\mathrm{mg}, 0.3 \mathrm{mmol}$ ) and benzoylformic acid $2 \mathrm{a}(45.0 \mathrm{mg}, 0.3 \mathrm{mmol}, 1$ equiv). Purified by column chromatography on silica gel (PE: EtOAC $=15: 1)$ to afford the pure product 3ra'.

White solid; yield: $38.2 \mathrm{mg}(40 \%)$; m.p. $133-134{ }^{\circ} \mathrm{C}$.

${ }^{1} \mathrm{H}$ NMR $\left(400 \mathrm{MHz}, \mathrm{CDCl}_{3}\right): \delta=7.50(\mathrm{~d}, J=8 \mathrm{~Hz}, 2 \mathrm{H}), 7.37-7.33(\mathrm{~m}, 1 \mathrm{H}), 7.28-7.23(\mathrm{~m}, 6 \mathrm{H}), 7.15-7.11$ $(\mathrm{m}, 1 \mathrm{H}), 6.96(\mathrm{~s}, 1 \mathrm{H}), 6.92(\mathrm{~d}, J=8.8 \mathrm{~Hz}, 2 \mathrm{H}), 6.82-6.80(\mathrm{~m}, 2 \mathrm{H}), 3.73(\mathrm{~s}, 3 \mathrm{H})$.

${ }^{13} \mathrm{CNMR}\left(150 \mathrm{MHz}, \mathrm{CDCl}_{3}\right): \delta=170.7,154.8,143.1,140.6,134.8,130.6$ 128.8, 128.6, 128.0, 126.2, $124.8,115.8,114.7,55.5$.

HRMS (ESI): $m / z[M+H]^{+}$calcd for $\mathrm{C}_{20} \mathrm{H}_{19} \mathrm{~N}_{2} \mathrm{O}_{2}^{+}$: 319.1441; found: 319.1443 . 
20) N'-(4-Chlorophenyl)-N-phenylbenzohydrazide (3sa)<smiles>O=C(c1ccccc1)N(Nc1ccc(Cl)cc1)c1ccccc1</smiles>

Prepared according to the general procedure from (E)-1-(4-chlorophenyl)-2-phenyldiazene 1s (65.0 $\mathrm{mg}, 0.3 \mathrm{mmol}$ ) and benzoylformic acid $2 \mathrm{a}(45.0 \mathrm{mg}, 0.3 \mathrm{mmol}, 1$ equiv). Purified by column chromatography on silica gel (PE: EtOAc $=15: 1)$ to afford the pure product 3sa.

White solid; yield: $65.8 \mathrm{mg}(68 \%) ;$ m.p. $150-151^{\circ} \mathrm{C}$

${ }^{1} \mathrm{H}$ NMR $\left(400 \mathrm{MHz}, \mathrm{CDCl}_{3}\right): \delta=7.49(\mathrm{~d}, J=8.0 \mathrm{~Hz}, 2 \mathrm{H}), 7.39-7.35(\mathrm{~m}, 1 \mathrm{H}), 7.28-7.23(\mathrm{~m}, 6 \mathrm{H}), 7.17(\mathrm{t}, J=$ $6.0 \mathrm{~Hz}, 4 \mathrm{H}), 6.83(\mathrm{~d}, J=8.4 \mathrm{~Hz}, 2 \mathrm{H})$.

${ }^{13} \mathrm{C} \mathrm{NMR}\left(150 \mathrm{MHz}, \mathrm{CDCl}_{3}\right): \delta=170.7,145.7,142.9,134.5,130.8,129.2,128.9,128.5,128.0,126.4$, $126.0,124.5,114.7$.

HRMS (ESI): $m / z[M+H]^{+}$calcd for $\mathrm{C}_{19} \mathrm{H}_{16} \mathrm{CIN}_{2} \mathrm{O}^{+}: 323.0946$; found: 323.0947 .

\section{1) $N$-(4-Chlorophenyl)-N'-phenylbenzohydrazide (3sa')}<smiles>O=C(c1ccccc1)N(Nc1ccccc1)c1ccc(Cl)cc1</smiles>

Prepared according to the general procedure from (E)-1-(4-chlorophenyl)-2-phenyldiazene 1 s (65.0 $\mathrm{mg}, 0.3 \mathrm{mmol}$ ) and benzoylformic acid $2 \mathrm{a}(45.0 \mathrm{mg}, 0.3 \mathrm{mmol}, 1$ equiv). Purified by column chromatography on silica gel $(\mathrm{PE}:$ EtOAc $=15: 1)$ to afford the pure product 3sa'.

White solid; yield: $23.2 \mathrm{mg}(24 \%)$; m.p. $139-140{ }^{\circ} \mathrm{C}$.

${ }^{1} \mathrm{H}$ NMR $\left(400 \mathrm{MHz}, \mathrm{CDCl}_{3}\right): \delta=7.52-7.49(\mathrm{~m}, 2 \mathrm{H}), 7.41-7.37(\mathrm{~m}, 1 \mathrm{H}), 7.31-7.21(\mathrm{~m}, 8 \mathrm{H}), 7.97-6.87(\mathrm{~m}$, $4 \mathrm{H})$.

${ }^{13} \mathrm{C} \mathrm{NMR}\left(150 \mathrm{MHz}, \mathrm{CDCl}_{3}\right): \delta=170.7,146.7,141.6,134.5,131.5,131.0,129.4,128.9,128.5,128.2$, $125.4,121.6,113.4$

HRMS (ESI): $m / z[M+H]^{+}$calcd for $\mathrm{C}_{19} \mathrm{H}_{16} \mathrm{ClN}_{2} \mathrm{O}^{+}$: 323.0946; found: 323.0948 .

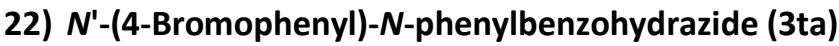


<smiles>O=C(c1ccccc1)N(Nc1ccccc1)c1ccc(Br)cc1</smiles>

Prepared according to the general procedure from (E)-1-(4-bromophenyl)-2-phenyldiazene 1t (78.3 $\mathrm{mg}, 0.3 \mathrm{mmol}$ ) and benzoylformic acid $2 \mathrm{a}(45.0 \mathrm{mg}, 0.3 \mathrm{mmol}, 1$ equiv). Purified by column chromatography on silica gel (PE: EtOAC $=15: 1)$ to afford the pure product 3ta.

White solid; yield: $69.4 \mathrm{mg}(63 \%) ;$ m.p. $150-151^{\circ} \mathrm{C}$

${ }^{1} \mathrm{H} \mathrm{NMR}\left(600 \mathrm{MHz}, \mathrm{CDCl}_{3}\right): \delta=7.50-7.48(\mathrm{~m}, 2 \mathrm{H}), 7.38-7.35(\mathrm{~m}, 1 \mathrm{H}), 7.31(\mathrm{~d}, J=8.4 \mathrm{~Hz}, 2 \mathrm{H}), 7.27-7.23$ (m, 6H), 7.16-7.13 (m, 2H), $6.77(\mathrm{~d}, J=8.4 \mathrm{~Hz}, 2 \mathrm{H})$.

${ }^{13} \mathrm{C} \mathrm{NMR}\left(150 \mathrm{MHz}, \mathrm{CDCl}_{3}\right): \delta=170.7,146.2,142.8,134.5,132.1,130.8,128.9,128.5,128.0,126.4$, $124.4,115.1,113.2$

HRMS (ESI): $m / z[M+H]^{+}$calcd for $\mathrm{C}_{19} \mathrm{H}_{16} \mathrm{BrN}_{2} \mathrm{O}^{+}: 367.0441$; found: 367.0443 .

\section{3) $N$-(4-Bromophenyl)-N'-phenylbenzohydrazide (3ta')}<smiles>O=C(c1ccccc1)N(Nc1ccccc1)c1ccc(Br)cc1</smiles>

Prepared according to the general procedure from (E)-1-(4-bromophenyl)-2-phenyldiazene 1t (78.3 $\mathrm{mg}, 0.3 \mathrm{mmol}$ ) and benzoylformic acid $2 \mathrm{a}(45.0 \mathrm{mg}, 0.3 \mathrm{mmol}, 1$ equiv). Purified by column chromatography on silica gel (PE: EtOAc $=15: 1)$ to afford the pure product 3ta'.

White solid; yield: $26.4 \mathrm{mg}$ (24\%); m.p. $134-135^{\circ} \mathrm{C}$.

${ }^{1} \mathrm{H} \mathrm{NMR}\left(400 \mathrm{MHz}, \mathrm{CDCl}_{3}\right) ; \delta=7.52-7.50(\mathrm{~m}, 2 \mathrm{H}), 7.44-7.33(\mathrm{~m}, 3 \mathrm{H}), 7.32-7.22(\mathrm{~m}, 4 \mathrm{H}), 7.16(\mathrm{~d}, J=8.8$ $H z, 2 H), 6.99-6.84(m, 4 H)$.

${ }^{13} \mathrm{C} \mathrm{NMR}\left(150 \mathrm{MHz}, \mathrm{CDCl}_{3}\right): \delta=170.7,146.7,142.2,134.5,131.9,131.0,129.4,128.5,128.2,125.7$, $121.6,119.3,113.4$

HRMS (ESI): $\mathrm{m} / \mathrm{z}[\mathrm{M}+\mathrm{H}]^{+}$calcd for $\mathrm{C}_{19} \mathrm{H}_{16} \mathrm{BrN}_{2} \mathrm{O}^{+}: 367.0441$; found: 367.0443 .

24) Ethyl 4-(2-benzoyl-2-phenylhydrazinyl)benzoate (3ua)<smiles>CCOC(=O)c1ccc(NN(C(=O)c2ccccc2)c2ccccc2)cc1</smiles> 
Prepared according to the general procedure from ethyl $(E)$-4-(phenyldiazenyl)benzoate $\mathbf{1 u}$ (76.3 $\mathrm{mg}$, $0.3 \mathrm{mmol}$ ) and benzoylformic acid $2 \mathrm{a}(45.0 \mathrm{mg}, 0.3 \mathrm{mmol}, 1$ equiv). Purified by column chromatography on silica gel $(\mathrm{PE}:$ EtOAc $=15: 1)$ to afford the pure product 3ua.

White solid; yield: $67 \mathrm{mg}(62 \%)$; m.p. $141-142{ }^{\circ} \mathrm{C}$.

${ }^{1} \mathrm{H}$ NMR $\left(600 \mathrm{MHz}, \mathrm{CDCl}_{3}\right): \delta=7.90(\mathrm{~d}, J=7.2 \mathrm{~Hz}, 2 \mathrm{H}), 7.49(\mathrm{~d}, J=7.2 \mathrm{~Hz}, 3 \mathrm{H}), 7.36-7.33(\mathrm{~m}, 1 \mathrm{H}), 7.27-$ $7.21(\mathrm{~m}, 6 \mathrm{H}), 7.14(\mathrm{t}, J=7.2 \mathrm{~Hz}, 1 \mathrm{H}), 6.85(\mathrm{~d}, J=7.8 \mathrm{~Hz}, 2 \mathrm{H}), 4.30(\mathrm{q}, J=7.2 \mathrm{~Hz}, 2 \mathrm{H}), 1.33(\mathrm{t}, J=7.2 \mathrm{~Hz}$, $3 \mathrm{H})$.

${ }^{13} \mathrm{C}$ NMR $\left(150 \mathrm{MHz}, \mathrm{CDCl}_{3}\right): \delta=170.9,166.3,150.9,142.6,134.5,131.3,130.9,128.9,128.4,128.0$, $126.4,124.2,122.7,112.0,60.4,14.3$.

HRMS (ESI): $m / z[M+H]^{+}$calcd for $\mathrm{C}_{22} \mathrm{H}_{21} \mathrm{~N}_{2} \mathrm{O}_{3}{ }^{+}: 361.1547$; found: 361.1550 .

\section{5) Ethyl 4-(1-benzoyl-2-phenylhydrazinyl)benzoate (3ua')}<smiles>CCOC(=O)c1ccc(N(Nc2ccccc2)C(=O)c2ccccc2)cc1</smiles>

Prepared according to the general procedure from ethyl $(E)$-4-(phenyldiazenyl)benzoate $1 \mathbf{u}(76.3 \mathrm{mg}$, $0.3 \mathrm{mmol}$ ) and benzoylformic acid $2 \mathrm{a}(45.0 \mathrm{mg}, 0.3 \mathrm{mmol}, 1$ equiv). Purified by column chromatography on silica gel (PE: EtOAc $=15: 1)$ to afford the pure product 3ua'.

White solid; yield: $31.3 \mathrm{mg}(29 \%)$; m.p. $129-130{ }^{\circ} \mathrm{C}$

${ }^{1} \mathrm{H}$ NMR $\left(600 \mathrm{MHz}, \mathrm{CDCl}_{3}\right): \delta=7.92(\mathrm{~d}, J=8.4 \mathrm{~Hz}, 2 \mathrm{H}), 7.52(\mathrm{~d}, J=7.2 \mathrm{~Hz}, 2 \mathrm{H}), 7.40(\mathrm{t}, J=7.5 \mathrm{~Hz}, 1 \mathrm{H})$, $7.35(\mathrm{~d}, J=9.0 \mathrm{~Hz}, 2 \mathrm{H}), 7.29(\mathrm{t}, J=7.5 \mathrm{~Hz}, 2 \mathrm{H}), 7.23(\mathrm{t}, J=7.8 \mathrm{~Hz}, 2 \mathrm{H}), 7.03-7.01(\mathrm{~m}, 1 \mathrm{H}), 6.92(\mathrm{~d}, J=$ $7.2 \mathrm{~Hz}, 1 \mathrm{H}), 6.88(\mathrm{~d}, J=7.8 \mathrm{~Hz}, 2 \mathrm{H}), 4.34(\mathrm{q}, J=7.2 \mathrm{~Hz}, 2 \mathrm{H}), 1.36(\mathrm{t}, J=7.2 \mathrm{~Hz}, 3 \mathrm{H})$.

${ }^{13} \mathrm{C} \mathrm{NMR}\left(150 \mathrm{MHz}, \mathrm{CDCl}_{3}\right): \delta=170.9,165.8,146.9,146.7,134.5,131.1,130.2,129.4,128.4,128.2$, $127.6,123.3,121.6,113.3,61.0,14.2$.

HRMS (ESI): $m / z[M+H]^{+}$calcd for $\mathrm{C}_{22} \mathrm{H}_{21} \mathrm{~N}_{2} \mathrm{O}_{3}{ }^{+}: 361.1547$; found: 361.1552 .

\section{6) $N$ '-(4-Cyanophenyl)-N-phenylbenzohydrazide (3va)}<smiles>N#Cc1ccc(NN(C(=O)c2ccccc2)c2ccccc2)cc1</smiles>

Prepared according to the general procedure from $(E)$-4-(phenyldiazenyl)benzonitrile $\mathbf{1 v}(62.1 \mathrm{mg}, 0.3$ 
$\mathrm{mmol}$ ) and benzoylformic acid $2 \mathrm{a}(45.0 \mathrm{mg}, 0.3 \mathrm{mmol}, 1$ equiv). Purified by column chromatography on silica gel (PE: EtOAC $=15: 1)$ to afford the pure product 3va.

White solid; yield: $53.6 \mathrm{mg}(57 \%)$; m.p. $146-147^{\circ} \mathrm{C}$

${ }^{1} \mathrm{H}$ NMR $\left(400 \mathrm{MHz}_{\mathrm{CDCl}}\right): \delta=7.57(\mathrm{~d}, J=7.8 \mathrm{~Hz}, 1 \mathrm{H}), 7.51-7.43(\mathrm{~m}, 4 \mathrm{H}), 7.41-7.36(\mathrm{~m}, 1 \mathrm{H}), 7.28-7.14$ $(\mathrm{m}, 7 \mathrm{H}), 6.89-6.86(\mathrm{~m}, 2 \mathrm{H})$.

${ }^{13} \mathrm{C}$ NMR $\left(150 \mathrm{MHz}, \mathrm{CDCl}_{3}\right): \delta=170.7,150.7,142.5,134.0,133.7,131.1,129.0,128.5,128.1,126.7$, $124.4,119.4,112.8,103.2$

HRMS (ESI): $m / z[M+H]^{+}$calcd for $\mathrm{C}_{20} \mathrm{H}_{16} \mathrm{~N}_{3} \mathrm{O}^{+}: 314.1288$; found: 314.1289 .

\section{7) $N$-(4-Cyanophenyl)-N'-phenylbenzohydrazide (3va')}<smiles>N#Cc1ccc(N(Nc2ccccc2)C(=O)c2ccccc2)cc1</smiles>

Prepared according to the general procedure from $(E)$-4-(phenyldiazenyl)benzonitrile $1 \mathbf{v}$ ( $62.1 \mathrm{mg}, 0.3$ $\mathrm{mmol}$ ) and benzoylformic acid $2 \mathrm{a}(45.0 \mathrm{mg}, 0.3 \mathrm{mmol}, 1$ equiv). Purified by column chromatography on silica gel (PE: EtOAc $=15: 1)$ to afford the pure product 3va'.

White solid; yield: $17.8 \mathrm{mg}(19 \%)$; m.p. $94-96^{\circ} \mathrm{C}$.

${ }^{1} \mathrm{H}$ NMR $\left(400 \mathrm{MHz}, \mathrm{CDCl}_{3}\right): \delta=7.54-7.49(\mathrm{~m}, 4 \mathrm{H}), 7.46-7.41(\mathrm{~m}, 3 \mathrm{H}), 7.31(\mathrm{t}, J=7.6 \mathrm{~Hz}, 2 \mathrm{H}), 7.26-7.11$ $(\mathrm{m}, 2 \mathrm{H}), 6.93(\mathrm{t}, J=9.2 \mathrm{~Hz}, 2 \mathrm{H}), 6.82(\mathrm{~d}, J=7.6 \mathrm{~Hz}, 2 \mathrm{H})$.

${ }^{13} \mathrm{C}$ NMR $\left(150 \mathrm{MHz}, \mathrm{CDCl}_{3}\right): \delta=171.1,146.8,146.2,134.2,132.8,131.5,129.5,128.4,128.2,123.5$, 121.8, 118.4, 113.1, 108.8.

HRMS (ESI): $m / z[M+H]^{+}$calcd for $\mathrm{C}_{20} \mathrm{H}_{16} \mathrm{~N}_{3} \mathrm{O}^{+}: 314.1288$; found: 314.1293 .

\section{8) $N^{\prime}$-(4-Nitrophenyl)-N-phenylbenzohydrazide (3wa)}<smiles>O=C(c1ccccc1)N(Nc1ccc([N+](=O)[O-])cc1)c1ccccc1</smiles>

Prepared according to the general procedure from (E)-1-(4-nitrophenyl)-2-phenyldiazene $\mathbf{1 w}$ (68.2 mg, $0.3 \mathrm{mmol}$ ) and benzoylformic acid $2 \mathrm{a}(45.0 \mathrm{mg}, 0.3 \mathrm{mmol}, 1$ equiv). Purified by column chromatography on silica gel $(\mathrm{PE}:$ EtOAc $=15: 1)$ to afford the pure product 3 wa. Yellow solid; yield: $61.0 \mathrm{mg}(61 \%) ;$ m.p. $184-185^{\circ} \mathrm{C}$. 
${ }^{1} \mathrm{H}$ NMR $\left(600 \mathrm{MHz}, \mathrm{CDCl}_{3}\right): \delta=8.06-8.04(\mathrm{~m}, 2 \mathrm{H}), 7.89(\mathrm{~d}, J=7.8 \mathrm{~Hz}, 1 \mathrm{H}), 7.46(\mathrm{~d}, J=7.2 \mathrm{~Hz}, 2 \mathrm{H}), 7.37(\mathrm{t}$, $J=7.5 \mathrm{~Hz}, 1 \mathrm{H}), 7.27-7.19(\mathrm{~m}, 6 \mathrm{H}), 7.18-7.15(\mathrm{~m}, 1 \mathrm{H}), 6.87-6.80(\mathrm{~m}, 2 \mathrm{H})$.

${ }^{13} \mathrm{C}$ NMR $\left(150 \mathrm{MHz}, \mathrm{CDCl}_{3}\right): \delta=170.8,152.5,142.2,140.9,133.9,131.2,129.1,128.5,128.2,126.9$, $125.9,124.4,111.7$.

HRMS (ESI): $m / z[M+H]^{+}$calcd for $\mathrm{C}_{19} \mathrm{H}_{16} \mathrm{~N}_{3} \mathrm{O}_{3}^{+}:$334.1168; found: 3334.1190 .

\section{9) $N$-(4-Nitrophenyl)-N'-phenylbenzohydrazide (3wa')}<smiles>O=C(c1ccccc1)N(Nc1ccccc1)c1ccc([N+](=O)[O-])cc1</smiles>

Prepared according to the general procedure from (E)-1-(4-nitrophenyl)-2-phenyldiazene $1 \mathbf{w}(68.2 \mathrm{mg}$, $0.3 \mathrm{mmol}$ ) and benzoylformic acid $2 \mathrm{a}(45.0 \mathrm{mg}, 0.3 \mathrm{mmol}, 1$ equiv). Purified by column chromatography on silica gel (PE: EtOAc $=15: 1)$ to afford the pure product $3 w a '$. Yellow solid; yield: $17.0 \mathrm{mg}(17 \%) ;$ m.p. $127-128^{\circ} \mathrm{C}$.

${ }^{1} \mathrm{H}$ NMR $\left(600 \mathrm{MHz}, \mathrm{CDCl}_{3}\right): \delta=8.12-8.09(\mathrm{~m}, 2 \mathrm{H}), 7.52-7.50(\mathrm{~m}, 2 \mathrm{H}), 7.48-7.42(\mathrm{~m}, 3 \mathrm{H}), 7.34-7.30(\mathrm{~m}$, $2 \mathrm{H}), 7.25-7.21(\mathrm{~m}, 2 \mathrm{H}), 6.95-6.89(\mathrm{~m}, 2 \mathrm{H}), 6.81(\mathrm{~d}, J=7.8 \mathrm{~Hz}, 2 \mathrm{H})$.

${ }^{13} \mathrm{C} \mathrm{NMR}\left(150 \mathrm{MHz}, \mathrm{CDCl}_{3}\right): \delta=171.2,148.5,146.1,144.6,134.1,131.6,129.6,128.5,128.2,124.4$, 123.1, 121.9, 113.2.

HRMS (ESI): $m / z[M+H]^{+}$calcd for $\mathrm{C}_{19} \mathrm{H}_{16} \mathrm{~N}_{3} \mathrm{O}_{3}^{+}$: 334.1168; found: 3334.1190 .

30) 4-Methyl-N,N'-diphenylbenzohydrazide (3ab) ${ }^{[4]}$<smiles>Cc1ccc(C(=O)N(Nc2ccccc2)c2ccccc2)cc1</smiles>

Prepared according to the general procedure from azobenzene $1 \mathrm{a}(36.4 \mathrm{mg}, 0.2 \mathrm{mmol})$ and 2-oxo-2-( $p$-tolyl)acetic acid 2b (32.8 mg, $0.2 \mathrm{mmol}, 1$ equiv). Purified by column chromatography on silica gel (PE: EtOAC $=10: 1)$ to afford the pure product 3 ab.

White solid; yield: $58.0 \mathrm{mg}(96 \%) ;$ m.p. $144-145^{\circ} \mathrm{C}$.

${ }^{1} \mathrm{H}$ NMR $\left(600 \mathrm{MHz}, \mathrm{CDCl}_{3}\right): \delta=7.45(\mathrm{~d}, J=6.6 \mathrm{~Hz}, 2 \mathrm{H}), 7.27-7.23(\mathrm{~m}, 6 \mathrm{H}), 7.15(\mathrm{t}, J=6.9 \mathrm{~Hz}, 1 \mathrm{H}), 7.08$ (d, J= $7.8 \mathrm{~Hz}, 2 \mathrm{H}), 7.02(\mathrm{~s}, 1 \mathrm{H}), 6.96(\mathrm{~d}, J=8.4 \mathrm{~Hz}, 2 \mathrm{H}), 6.93(\mathrm{t}, J=7.5 \mathrm{~Hz}, 1 \mathrm{H}), 2.32(\mathrm{~s}, 3 \mathrm{H})$.

${ }^{13} \mathrm{C}$ NMR $\left(150 \mathrm{MHz}, \mathrm{CDCl}_{3}\right): \delta=170.7,147.3,143.5,141.2,131.8,129.2,128.8,128.8,128.7,126.0$, 


\section{1) 4-Isopropyl-N,N'-diphenylbenzohydrazide (3ac)}<smiles>CC(C)c1ccc(C(=O)N(Nc2ccccc2)c2ccccc2)cc1</smiles>

Prepared according to the general procedure from azobenzene 1a $(36.4 \mathrm{mg}, 0.2 \mathrm{mmol})$ and 2-(4-Isopropylphenyl)-2-oxoacetic acid 2c (38.4 mg, $0.2 \mathrm{mmol}, 1$ equiv). Purified by column chromatography on silica gel (PE: EtOAc $=10: 1)$ to afford the pure product 3ac.

White solid; yield: $61.4 \mathrm{mg}(93 \%)$; m.p. $141-142{ }^{\circ} \mathrm{C}$.

${ }^{1} \mathrm{H} \mathrm{NMR}\left(600 \mathrm{MHz}, \mathrm{CDCl}_{3}\right): \delta=7.49(\mathrm{~d}, J=8.4 \mathrm{~Hz}, 2 \mathrm{H}), 7.29(\mathrm{~d}, J=7.8 \mathrm{~Hz}, 2 \mathrm{H}), 7.26-7.22(\mathrm{~m}, 4 \mathrm{H}), 7.15-$ $7.12(\mathrm{~m}, 3 \mathrm{H}), 6.99(\mathrm{~s}, 1 \mathrm{H}), 6.95(\mathrm{~d}, J=7.8 \mathrm{~Hz}, 2 \mathrm{H}), 6.91(\mathrm{t}, J=7.5 \mathrm{~Hz}, 1 \mathrm{H}), 2.90-2.85(\mathrm{~m}, 1 \mathrm{H}), 1.23(\mathrm{~s}$, $3 \mathrm{H}), 1.21(\mathrm{~s}, 3 \mathrm{H})$.

${ }^{13} \mathrm{C} \mathrm{NMR}\left(150 \mathrm{MHz}, \mathrm{CDCl}_{3}\right): \delta=170.8,152.0,147.3,143.6,132.2,129.3,128.9,128.8,126.1,126.0$, $124.5,121.3,113.6,34.0,23.6$.

HRMS (ESI): $m / z[M+H]^{+}$calcd for $\mathrm{C}_{22} \mathrm{H}_{23} \mathrm{~N}_{2} \mathrm{O}^{+}: 331.1805$; found: 331.1805 .

\section{2) 4-Butyl- $N, N^{\prime}$-diphenylbenzohydrazide (3ad)}<smiles>CC(C)(C)c1ccc(C(=O)N(Nc2ccccc2)c2ccccc2)cc1</smiles>

Prepared according to the general procedure from azobenzene $1 \mathrm{a}(36.4 \mathrm{mg}, 0.2 \mathrm{mmol})$ and 2-(4-butylphenyl)-2-oxoacetic acid $2 \mathrm{~d}(41.2 \mathrm{mg}, 0.2 \mathrm{mmol}, 1$ equiv). Purified by column chromatography on silica gel (PE: EtOAC $=10: 1)$ to afford the pure product 3ad.

White solid; yield: $60.6 \mathrm{mg}(88 \%)$; m.p. $112-113^{\circ} \mathrm{C}$.

${ }^{1} \mathrm{H} \mathrm{NMR}\left(600 \mathrm{MHz}, \mathrm{CDCl}_{3}\right): \delta=7.47(\mathrm{~d}, J=7.8 \mathrm{~Hz}, 2 \mathrm{H}), 7.28(\mathrm{~d}, J=7.8 \mathrm{~Hz}, 2 \mathrm{H}), 7.26-7.23(\mathrm{~m}, 4 \mathrm{H}), 7.16-$ $7.13(\mathrm{~m}, 1 \mathrm{H}), 7.08(\mathrm{~d}, J=8.4 \mathrm{~Hz}, 2 \mathrm{H}), 7.04(\mathrm{~s}, 1 \mathrm{H}), 6.95(\mathrm{~d}, J=7.8 \mathrm{~Hz}, 2 \mathrm{H}), 6.91(\mathrm{~d}, J=7.2 \mathrm{~Hz}, 1 \mathrm{H}) 2.59(\mathrm{t}$, $J=7.8 \mathrm{~Hz}, 2 \mathrm{H}), 1.60-1.54(\mathrm{~m}, 2 \mathrm{H}), 1.36-1.30(\mathrm{~m}, 2 \mathrm{H}), 0.92(\mathrm{t}, J=7.2 \mathrm{~Hz}, 3 \mathrm{H})$.

${ }^{13} \mathrm{C} \mathrm{NMR}\left(150 \mathrm{MHz}, \mathrm{CDCl}_{3}\right): \delta=170.8,147.3,146.1,143.5,131.9,129.2,128.8,128.7,128.0,126.0$, $124.4,121.2,113.6,35.4,33.1,22.2,13.8$. 
HRMS (ESI): $m / z[M+H]^{+}$calcd for $\mathrm{C}_{22} \mathrm{H}_{25} \mathrm{~N}_{2} \mathrm{O}^{+}: 345.1961$; found: 345.1961 .

33) 4-Methoxy- $N, N^{\prime}$-diphenylbenzohydrazide (3ae) ${ }^{[4]}$<smiles>COc1ccc(C(=O)N(Nc2ccccc2)c2ccccc2)cc1</smiles>

Prepared according to the general procedure from azobenzene 1a $(36.4 \mathrm{mg}, 0.2 \mathrm{mmol}$ ) and 2-(4-methoxyphenyl)-2-oxoacetic acid 2 e $(36.0 \mathrm{mg}, 0.2 \mathrm{mmol}, 1$ equiv). Purified by column chromatography on silica gel (PE: EtOAc $=10: 1)$ to afford the pure product 3ae.

White solid; yield: $55.4 \mathrm{mg}(87 \%)$; m.p. $139-140{ }^{\circ} \mathrm{C}$.

${ }^{1} \mathrm{H}$ NMR $\left(600 \mathrm{MHz}, \mathrm{CDCl}_{3}\right): \delta=7.55-7.51(\mathrm{~m}, 2 \mathrm{H}), 7.26-7.23(\mathrm{~m}, 6 \mathrm{H}), 7.16-7.12(\mathrm{~m}, 1 \mathrm{H}), 7.00(\mathrm{~s}, 1 \mathrm{H})$, $6.97(\mathrm{~d}, J=7.8 \mathrm{~Hz}, 2 \mathrm{H}) 6.91(\mathrm{t}, J=7.8 \mathrm{~Hz}, 1 \mathrm{H}), 6.78-6.75(\mathrm{~m}, 2 \mathrm{H}), 3.78(\mathrm{~s}, 3 \mathrm{H})$.

${ }^{13} \mathrm{C}$ NMR $\left(150 \mathrm{MHz}, \mathrm{CDCl}_{3}\right): \delta=170.3,161.7,147.5,143.9,131.0,129.3,128.9,126.6,126.0,124.5$, $121.3,113.5,113.3,55.3$.

34) 4-(Benzyloxy)- $N, N^{\prime}$-diphenylbenzohydrazide (3af)<smiles>O=C(c1ccc(OCc2ccccc2)cc1)N(Nc1ccccc1)c1ccccc1</smiles>

Prepared according to the general procedure from azobenzene 1a $(36.4 \mathrm{mg}, 0.2 \mathrm{mmol}$ ) and 2-(4-(benzyloxy)phenyl)-2-oxoacetic acid $2 \mathrm{f}$ ( $51.3 \mathrm{mg}, 0.2 \mathrm{mmol}, 1$ equiv). Purified by column chromatography on silica gel (PE: EtOAc $=10: 1$ ) to afford the pure product 3af.

White solid; yield: $66.2 \mathrm{mg}(84 \%) ;$ m.p. $153-154^{\circ} \mathrm{C}$.

${ }^{1} \mathrm{H} \mathrm{NMR}\left(600 \mathrm{MHz}, \mathrm{CDCl}_{3}\right): \delta=7.55-7.54(\mathrm{~m}, 2 \mathrm{H}), 7.41-7.37(\mathrm{~m}, 4 \mathrm{H}), 7.36-7.32(\mathrm{~m}, 1 \mathrm{H}), 7.27-7.25(\mathrm{~m}$, $5 \mathrm{H}), 7.24(\mathrm{~s}, 1 \mathrm{H}), 7.16-7.13(\mathrm{~m}, 1 \mathrm{H}), 7.03-7.01(\mathrm{~m}, 1 \mathrm{H}), 6.97(\mathrm{~d}, J=7.8 \mathrm{~Hz}, 2 \mathrm{H}), 6.93(\mathrm{t}, J=7.5 \mathrm{~Hz}, 1 \mathrm{H})$, $6.85(\mathrm{~d}, J=8.4 \mathrm{~Hz}, 2 \mathrm{H}), 5.04(\mathrm{~s}, 2 \mathrm{H})$.

${ }^{13} \mathrm{C} \mathrm{NMR}\left(150 \mathrm{MHz}, \mathrm{CDCl}_{3}\right): \delta=170.2,160.8,147.4,143.8,136.2,131.0,129.3,128.8,128.6,128.1$, $127.4,126.9,126.0,124.5,121.3,114.2,113.5,69.9$.

HRMS (ESI): $\mathrm{m} / 2[\mathrm{M}+\mathrm{H}]^{+}$calcd for $\mathrm{C}_{26} \mathrm{H}_{23} \mathrm{~N}_{2} \mathrm{O}_{2}^{+}: 395.1754$; found: 395.1755 .

35) 4-Fluoro- $N, N$ '-diphenylbenzohydrazide (3ag) 
<smiles>O=C(c1ccc(F)cc1)N(Nc1ccccc1)c1ccccc1</smiles>

Prepared according to the general procedure from azobenzene $1 \mathrm{a}(36.4 \mathrm{mg}, 0.2 \mathrm{mmol})$ and 2-(4-fluorophenyl)-2-oxoacetic acid $2 \mathrm{~g}(33.6 \mathrm{mg}, 0.2 \mathrm{mmol}, 1$ equiv). Purified by column chromatography on silica gel (PE: EtOAc $=10: 1)$ to afford the pure product 3ag.

White solid; yield: $58.1 \mathrm{mg}$ (95\%); m.p. $162-164^{\circ} \mathrm{C}$.

${ }^{1} \mathrm{H}$ NMR $\left(600 \mathrm{MHz}, \mathrm{CDCl}_{3}\right): \delta=7.56-7.53(\mathrm{~m}, 2 \mathrm{H}), 7.27-7.23(\mathrm{~m}, 6 \mathrm{H}), 7.18-7.15(\mathrm{~m}, 1 \mathrm{H}), 6.9(\mathrm{~s}, 1 \mathrm{H})$, $6.97-6.91(m, 5 H)$.

${ }^{13} \mathrm{CNMR}\left(150 \mathrm{MHz}, \mathrm{CDCl}_{3}\right): \delta=169.7,164.8(\mathrm{~d}, J=250.5 \mathrm{~Hz}), 146.9,143.0,131.2(\mathrm{~d}, J=9.0 \mathrm{~Hz}), 130.8$, (d, $J=3.0 \mathrm{~Hz}), 129.3,128.9,126.4,124.5,121.4,115.2(\mathrm{~d}, J=21.0 \mathrm{~Hz}), 113.5$.

HRMS (ESI): $\mathrm{m} / z[\mathrm{M}+\mathrm{H}]^{+}$calcd for $\mathrm{C}_{19} \mathrm{H}_{16} \mathrm{FN}_{2} \mathrm{O}^{+}: 307.1241$; found: 307.1241 .

\section{6) 4-Chloro-N,N'-diphenylbenzohydrazide (3ah)}<smiles>O=C(c1ccc(Cl)cc1)N(Nc1ccccc1)c1ccccc1</smiles>

Prepared according to the general procedure from azobenzene $1 \mathrm{a}(36.4 \mathrm{mg}, 0.2 \mathrm{mmol})$ and 2-(4-chlorophenyl)-2-oxoacetic acid $2 \mathrm{~h}$ (36.7 $\mathrm{mg}, 0.2 \mathrm{mmol}, 1$ equiv). Purified by column chromatography on silica gel (PE: EtOAc $=10: 1)$ to afford the pure product 3ah.

White solid; yield: $60.6 \mathrm{mg}$ (94\%); m.p. $168-169^{\circ} \mathrm{C}$.

${ }^{1} \mathrm{H}$ NMR $\left(600 \mathrm{MHz}, \mathrm{CDCl}_{3}\right): \delta=7.48-7.46(\mathrm{~m}, 2 \mathrm{H}), 7.30-7.26(\mathrm{~m}, 4 \mathrm{H}), 7.25-7.23(\mathrm{~m}, 4 \mathrm{H}), 7.18-7.15(\mathrm{~m}$, $1 \mathrm{H}), 7.03(\mathrm{~s}, 1 \mathrm{H}), 6.94-6.90(\mathrm{~m}, 3 \mathrm{H})$,

${ }^{13} \mathrm{C} \mathrm{NMR}\left(150 \mathrm{MHz}, \mathrm{CDCl}_{3}\right): \delta=169.8,146.7,142.7,136.8,133.2,130.0,129.3,128.9,128.2,126.4$, $124.4,121.4,113.4$

HRMS (ESI): $\mathrm{m} / \mathrm{z}[\mathrm{M}+\mathrm{H}]^{+}$calcd for $\mathrm{C}_{19} \mathrm{H}_{16} \mathrm{CIN}_{2} \mathrm{O}^{+}: 323.0946$; found: 323.0947 .

37) 4-Bromo-N,N'-diphenylbenzohydrazide (3ai) ${ }^{[4]}$ 
<smiles>O=C(c1ccc(Br)cc1)N(Nc1ccccc1)c1ccccc1</smiles>

Prepared according to the general procedure from azobenzene $1 \mathrm{a}(36.4 \mathrm{mg}, 0.2 \mathrm{mmol})$ and 2-(4-bromophenyl)-2-oxoacetic acid 2i $(45.8 \mathrm{mg}, 0.2 \mathrm{mmol}, 1$ equiv). Purified by column chromatography on silica gel $(\mathrm{PE}: \mathrm{EtOAC}=10: 1)$ to afford the pure product 3ai.

White solid; yield: $67.5 \mathrm{mg}(92 \%) ;$ m.p. $176-177^{\circ} \mathrm{C}$.

${ }^{1} \mathrm{H}$ NMR $\left(600 \mathrm{MHz}, \mathrm{CDCl}_{3}\right): \delta=7.40(\mathrm{~s}, 4 \mathrm{H}), 7.28-7.23(\mathrm{~m}, 6 \mathrm{H}), 7.19-7.17(\mathrm{~m}, 1 \mathrm{H}), 6.98(\mathrm{~s}, 1 \mathrm{H}), 6.94-$ $6.90(\mathrm{~m}, 3 \mathrm{H})$.

${ }^{13} \mathrm{C}$ NMR $\left(150 \mathrm{MHz}, \mathrm{CDCl}_{3}\right): \delta=169.9,146.7,142.7,133.6,131.2,130.2,129.4,129.0,126.5,125.3$, $124.4,121.5,113.4$

HRMS (ESI): $\mathrm{m} / \mathrm{z}[\mathrm{M}+\mathrm{H}]^{+}$calcd for $\mathrm{C}_{19} \mathrm{H}_{16} \mathrm{BrN}_{2} \mathrm{O}^{+}: 367.0441$; found: 367.0437 .

\section{8) $N, N^{\prime}$-Biphenyl-4-(trifluoromethyl)benzohydrazide (3aj)}<smiles>O=C(c1ccc(C(F)(F)F)cc1)N(Nc1ccccc1)c1ccccc1</smiles>

Prepared according to the general procedure from azobenzene 1a $(36.4 \mathrm{mg}, 0.2 \mathrm{mmol}$ ) and 2-oxo-2-(4-(trifluoromethyl)phenyl)acetic acid $2 \mathrm{j}(43.6 \mathrm{mg}, 0.2 \mathrm{mmol}, 1$ equiv). Purified by column chromatography on silica gel $(\mathrm{PE}:$ EtOAc $=10: 1)$ to afford the pure product 3aj.

White solid; yield: $63.4 \mathrm{mg}(89 \%)$; m.p. $120-121^{\circ} \mathrm{C}$.

${ }^{1} \mathrm{H} \mathrm{NMR}\left(600 \mathrm{MHz}, \mathrm{CDCl}_{3}\right): \delta=7.62(\mathrm{~d}, J=8.4 \mathrm{~Hz}, 2 \mathrm{H}), 7.53(\mathrm{~d}, J=8.4 \mathrm{~Hz}, 2 \mathrm{H}), 7.32-7.22(\mathrm{~m}, 6 \mathrm{H}), 7.20-$ $7.17(\mathrm{~m}, 1 \mathrm{H}), 6.98(\mathrm{~s}, 1 \mathrm{H}), 6.94-6.87(\mathrm{~m}, 3 \mathrm{H})$.

${ }^{13} \mathrm{C} \mathrm{NMR}\left(150 \mathrm{MHz}, \mathrm{CDCl}_{3}\right): \delta=169.9,146.3,141.9,138.6,132.2(\mathrm{q}, J=33.0 \mathrm{~Hz}), 129.3(\mathrm{~d}, J=58.5 \mathrm{~Hz})$, $128.5,126.6,124.9(\mathrm{~d}, J=3.0 \mathrm{~Hz}), 124.4,124.2,122.6,121.3,113.2$.

HRMS (ESI): $\mathrm{m} / \mathrm{z}[\mathrm{M}+\mathrm{H}]^{+}$calcd for $\mathrm{C}_{20} \mathrm{H}_{16} \mathrm{~F}_{3} \mathrm{~N}_{2} \mathrm{O}^{+}: 357.1209$; found: 357.1210 .

39) 4-(Methylsulfonyl)-N,N'-diphenylbenzohydrazide (3ak)<smiles>CS(=O)(=O)c1ccc(C(=O)N(Nc2ccccc2)c2ccccc2)cc1</smiles> 
Prepared according to the general procedure from azobenzene 1a $(36.4 \mathrm{mg}, 0.2 \mathrm{mmol}$ ) and 2-(4-(methylsulfonyl)phenyl)-2-oxoacetic acid $2 \mathbf{k}(45.6 \mathrm{mg}, 0.2 \mathrm{mmol}, 1$ equiv). Purified by column chromatography on silica gel $(\mathrm{PE}:$ EtOAC $=10: 1)$ to afford the pure product 3ak.

White solid; yield: $65.9 \mathrm{mg}(90 \%)$; m.p. $133-134{ }^{\circ} \mathrm{C}$.

${ }^{1} \mathrm{H}$ NMR $\left(600 \mathrm{MHz}, \mathrm{DMSO}-d_{6}\right): \delta=9.03(\mathrm{~s}, 1 \mathrm{H}), 7.91(\mathrm{~d}, J=8.4 \mathrm{~Hz}, 2 \mathrm{H}), 7.80(\mathrm{~d}, J=7.8 \mathrm{~Hz}, 2 \mathrm{H}), 7.59(\mathrm{~d}, J$ $=7.2 \mathrm{~Hz}, 2 \mathrm{H}), 7.36(\mathrm{t}, J=7.8 \mathrm{~Hz}, 2 \mathrm{H}), 7.20-7.17(\mathrm{~m}, 1 \mathrm{H}), 7.14(\mathrm{t}, J=7.8 \mathrm{~Hz}, 2 \mathrm{H}), 6.80(\mathrm{~d}, J=8.4 \mathrm{~Hz}, 2 \mathrm{H})$, $6.72(t, J=6.9 \mathrm{~Hz}, 1 \mathrm{H}), 3.21(\mathrm{~s}, 3 \mathrm{H})$.

${ }^{13} \mathrm{C}$ NMR $\left(150 \mathrm{MHz}\right.$, DMSO- $\left.d_{6}\right): \delta=169.9,145.8,141.6,141.32,141.27,129.9,128.4,127.7,126.3$, $126.0,124.0,119.5,112.4,43.1$.

HRMS (ESI): $m / z[M+H]^{+}$calcd for $\mathrm{C}_{20} \mathrm{H}_{19} \mathrm{~N}_{2} \mathrm{O}_{3} \mathrm{~S}^{+}: 367.1111$; found: 367.1115 .

\section{0) 3-Methyl-N,N'-diphenylbenzohydrazide (3al)}<smiles>Cc1cccc(C(=O)N(Nc2ccccc2)c2ccccc2)c1</smiles>

Prepared according to the general procedure from azobenzene 1a (36.4 mg, $0.2 \mathrm{mmol}$ ) and 2-oxo-2-(m-tolyl)acetic acid $2 \mathbf{l}(32.8 \mathrm{mg}, 0.2 \mathrm{mmol}, 1$ equiv). Purified by column chromatography on silica gel (PE: EtOAc $=10: 1)$ to afford the pure product 3al.

White solid; yield: $55.6 \mathrm{mg}(92 \%) ;$ m.p. $112-113^{\circ} \mathrm{C}$.

${ }^{1} \mathrm{H}$ NMR $\left(600 \mathrm{MHz}, \mathrm{CDCl}_{3}\right): \delta=7.40(\mathrm{~s}, 1 \mathrm{H}), 7.33-7.22(\mathrm{~m}, 7 \mathrm{H}), 7.20-7.12(\mathrm{~m}, 3 \mathrm{H}), 7.09(\mathrm{~s}, 1 \mathrm{H}), 6.98-$ $6.89(\mathrm{~m}, 3 \mathrm{H}), 2.28(\mathrm{~s}, 3 \mathrm{H})$.

${ }^{13} \mathrm{CNMR}\left(150 \mathrm{MHz}, \mathrm{CDCl}_{3}\right): \delta=170.9,147.1,143.2,137.8,134.7,131.4,129.18,129.15,128.7,127.7$, $126.0,125.5,124.4,121.1,113.4,21.2$.

HRMS (ESI): $m / z[M+H]^{+}$calcd for $\mathrm{C}_{20} \mathrm{H}_{19} \mathrm{~N}_{2} \mathrm{O}^{+}$: 303.1492; found: 303.1494 .

\section{1) 3-Chloro- $N, N^{\prime}$-diphenylbenzohydrazide (3am)}<smiles>O=C(c1cccc(Cl)c1)N(Nc1ccccc1)c1ccccc1</smiles>

Prepared according to the general procedure from azobenzene $1 \mathrm{a}(36.4 \mathrm{mg}, 0.2 \mathrm{mmol}$ ) and 2-(3-chlorophenyl)-2-oxoacetic acid $2 \mathrm{~m}$ (36.9 $\mathrm{mg}, 0.2 \mathrm{mmol}, 1$ equiv). Purified by column 
chromatography on silica gel (PE: EtOAC $=10: 1)$ to afford the pure product 3am.

White solid; yield: $60.0 \mathrm{mg}(93 \%) ;$ m.p. $115-116^{\circ} \mathrm{C}$.

${ }^{1} \mathrm{H} \mathrm{NMR}\left(600 \mathrm{MHz}, \mathrm{CDCl}_{3}\right): \delta=7.54(\mathrm{t}, J=1.8 \mathrm{~Hz}, 1 \mathrm{H}), 7.36-7.23(\mathrm{~m}, 8 \mathrm{H}), 7.19-7.16(\mathrm{~m}, 2 \mathrm{H}), 6.99(\mathrm{~s}$, $1 \mathrm{H}), 6.94-6.87(\mathrm{~m}, 3 \mathrm{H})$.

${ }^{13} \mathrm{C} \mathrm{NMR}\left(150 \mathrm{MHz}, \mathrm{CDCl}_{3}\right): \delta=169.5,146.4,142.2,136.6,133.8,130.5,129.2,129.1,128.8,128.4$, $126.4,126.3,124.2,121.1,113.2$.

HRMS (ESI): $m / z[M+H]^{+}$calcd for $\mathrm{C}_{19} \mathrm{H}_{16} \mathrm{CIN}_{2} \mathrm{O}^{+}:$323.0946; found: 323.0949 .

42) 2-Chloro- $N, N^{\prime}-$ diphenylbenzohydrazide (3an) ${ }^{[4]}$<smiles>O=C(c1ccccc1Cl)N(Nc1ccccc1)c1ccccc1</smiles>

Prepared according to the general procedure from azobenzene $1 \mathrm{a}(36.4 \mathrm{mg}, 0.2 \mathrm{mmol})$ and 2-(2-chlorophenyl)-2-oxoacetic acid $2 \mathrm{n}$ (36.9 $\mathrm{mg}, 0.2 \mathrm{mmol}, 1$ equiv). Purified by column chromatography on silica gel (PE: EtOAc $=10: 1)$ to afford the pure product 3an.

White solid; yield: $58.1 \mathrm{mg}(90 \%)$; m.p. $106-108^{\circ} \mathrm{C}$.

${ }^{1} \mathrm{H}$ NMR $\left(400 \mathrm{MHz}, \mathrm{CDCl}_{3}\right): \delta=7.66(\mathrm{~s}, 1 \mathrm{H}), 7.38-6.54(\mathrm{~m}, 14 \mathrm{H})$.

${ }^{13} \mathrm{C}$ NMR $\left(100 \mathrm{MHz}, \mathrm{CDCl}_{3}\right): \delta=170.6,145.6,140.7,136.5,129.9,129.0,128.6,127.2,126.5,125.1$, $122.7,121.5,120.6,114.1,112.5$.

HRMS (ESI): $\mathrm{m} / \mathrm{z}[\mathrm{M}+\mathrm{H}]^{+}$calcd for $\mathrm{C}_{19} \mathrm{H}_{16} \mathrm{ClN}_{2} \mathrm{O}^{+}$: 323.0946; found: 323.0939 .

\section{3) 3,4-Dimethoxy- $N, N^{\prime}$-diphenylbenzohydrazide (3ao)}<smiles>COc1ccc(C(=O)N(Nc2ccccc2)c2ccccc2)cc1OC</smiles>

Prepared according to the general procedure from azobenzene 1a (36.4 mg, $0.2 \mathrm{mmol}$ ) and 2-(3,4-dimethoxyphenyl)-2-oxoacetic acid $20(42.0 \mathrm{mg}, 0.2 \mathrm{mmol}, 1$ equiv). Purified by column chromatography on silica gel (PE: EtOAc $=10: 1)$ to afford the pure product 3ao.

White solid; yield: $55.0 \mathrm{mg}(79 \%) ;$ m.p. $69-71^{\circ} \mathrm{C}$.

${ }^{1} \mathrm{H}$ NMR $\left(400 \mathrm{MHz}, \mathrm{CDCl}_{3}\right): \delta=7.27-7.22(\mathrm{~m}, 6 \mathrm{H}), 7.17-7.13(\mathrm{~m}, 2 \mathrm{H}), 7.00(\mathrm{~s}, 1 \mathrm{H}), 7.08(\mathrm{~d}, J=1.6 \mathrm{~Hz}$, $1 \mathrm{H}), 6.96-6.88(\mathrm{~m}, 3 \mathrm{H}), 6.72(\mathrm{~d}, J=8.4 \mathrm{~Hz}, 1 \mathrm{H}), 3.84(\mathrm{~s}, 3 \mathrm{H}), 3.63(\mathrm{~s}, 3 \mathrm{H})$. 
${ }^{13} \mathrm{C}$ NMR $\left(150 \mathrm{MHz}, \mathrm{CDCl}_{3}\right): \delta=170.2,151.2,148.1,147.3,143.8,129.3,128.9,126.5,126.1,124.5$, $122.9,121.3,113.4,112.0,110.0,55.8,55.6$.

HRMS (ESI): $\mathrm{m} / \mathrm{z}[\mathrm{M}+\mathrm{H}]^{+}$calcd for $\mathrm{C}_{21} \mathrm{H}_{21} \mathrm{~N}_{2} \mathrm{O}_{3}^{+}: 349.1547$; found: 349.1550 .

44) N,N'-Diphenyl-2-naphthohydrazide (3ap)<smiles>O=C(c1ccc2ccccc2c1)N(Nc1ccccc1)c1ccccc1</smiles>

Prepared according to the general procedure from azobenzene 1a (36.4 mg, $0.2 \mathrm{mmol}$ ) and 2-(naphthalen-2-yl)-2-oxoacetic acid $2 \mathrm{p}(40.0 \mathrm{mg}, 0.2 \mathrm{mmol}, 1$ equiv). Purified by column chromatography on silica gel (PE: EtOAC $=10: 1)$ to afford the pure product 3ap.

White solid; yield: $60.9 \mathrm{mg}(90 \%)$; m.p. $181-183^{\circ} \mathrm{C}$.

${ }^{1} \mathrm{H}$ NMR $\left(600 \mathrm{MHz}, \mathrm{DMSO}-d_{6}\right): \delta=9.09(\mathrm{~s}, 1 \mathrm{H}), 8.17(\mathrm{~s}, 1 \mathrm{H}), 7.89(\mathrm{~d}, J=7.8 \mathrm{~Hz}, 2 \mathrm{H}), 7.83(\mathrm{~d}, J=9 \mathrm{~Hz}$, 1H), $7.63(\mathrm{~d}, J=8.4 \mathrm{~Hz}, 1 \mathrm{H}), 7.56-7.51(\mathrm{~m}, 4 \mathrm{H}), 7.33(\mathrm{t}, J=8.1 \mathrm{~Hz}, 2 \mathrm{H}), 7.17-7.13(\mathrm{~m}, 3 \mathrm{H}), 6.81(\mathrm{~d}, J=$ $7.8 \mathrm{~Hz}, 2 \mathrm{H}), 6.70(\mathrm{t}, J=7.2 \mathrm{~Hz}, 1 \mathrm{H})$.

${ }^{13} \mathrm{C}$ NMR $\left(150 \mathrm{MHz}, \mathrm{DMSO}-d_{6}\right): \delta=171.1,146.5,141.8,133.7,133.4,132.0,129.1,128.6,128.4,127.6$, $127.5,127.3,127.0,126.6,125.9,124.6,124.2,119.3,112.2$.

HRMS (ESI): $m / z[M+H]^{+}$calcd for $\mathrm{C}_{23} \mathrm{H}_{19} \mathrm{~N}_{2} \mathrm{O}^{+}: 339.1492$; found: 339.1492 .

\section{5) $N, N$ '-Diphenylthiophene-2-carbohydrazide (3aq)}<smiles>O=C(c1cccs1)N(Nc1ccccc1)c1ccccc1</smiles>

Prepared according to the general procedure from azobenzene $1 \mathrm{a}(36.4 \mathrm{mg}, 0.2 \mathrm{mmol})$ and 2-oxo-2-(thiophen-2-yl)acetic acid $2 q(31.2 \mathrm{mg}, 0.2 \mathrm{mmol}, 1$ equiv). Purified by column chromatography on silica gel $(P E:$ EtOAc $=10: 1)$ to afford the pure product 3aq.

White solid; yield: $25.9 \mathrm{mg}(44 \%)$; m.p. $220-221^{\circ} \mathrm{C}$.

${ }^{1} \mathrm{H}$ NMR $\left(600 \mathrm{MHz}\right.$, DMSO- $\left.d_{6}\right): \delta=9.26(\mathrm{~s}, 1 \mathrm{H}), 7.93(\mathrm{~d}, J=4.2 \mathrm{~Hz}, 1 \mathrm{H}), 7.76-7.75(\mathrm{~m}, 1 \mathrm{H}), 7.59(\mathrm{~d}, J=$ $8.4 \mathrm{~Hz}, 2 \mathrm{H}), 7.33(\mathrm{t}, J=7.8 \mathrm{~Hz}, 2 \mathrm{H}), 7.16-7.10(\mathrm{~m}, 4 \mathrm{H}), 6.80(\mathrm{~d}, J=8.4 \mathrm{~Hz}, 2 \mathrm{H}), 6.75-6.72(\mathrm{~m}, 1 \mathrm{H})$.

${ }^{13} \mathrm{C}$ NMR $\left(150 \mathrm{MHz}, \mathrm{DMSO}-d_{6}\right): \delta=163.2,146.0,141.2,135.9,135.3,133.5,129.7,128.9,127.1,126.4$, $124.8,120.7,113.7$. 
HRMS (ESI): $m / z[M+H]^{+}$calcd for $\mathrm{C}_{17} \mathrm{H}_{15} \mathrm{~N}_{2} \mathrm{OS}^{+}$: 295.0900; found: 295.0902 .

\section{6) $N, N^{\prime}$-Diphenylacetohydrazide (3ar)}<smiles>CC(=O)N(Nc1ccccc1)c1ccccc1</smiles>

Prepared according to the general procedure from azobenzene $1 \mathrm{a}(36.4 \mathrm{mg}, 0.2 \mathrm{mmol}$ ) and 2-oxopropanoic acid $2 \mathrm{r}(31.2 \mathrm{mg}, 0.2 \mathrm{mmol}, 1$ equiv). Purified by column chromatography on silica gel $(P E: E t O A C=10: 1)$ to afford the pure product 3 ar.

White solid; yield: $13.6 \mathrm{mg}(30 \%) ;$ m.p. $168-170^{\circ} \mathrm{C}$.

${ }^{1} \mathrm{H}$ NMR $\left(600 \mathrm{MHz}, \mathrm{DMSO}-d_{6}\right): \delta=8.98(\mathrm{~s}, 1 \mathrm{H}), 7.54(\mathrm{~d}, J=7.8 \mathrm{~Hz}, 2 \mathrm{H}), 7.31(\mathrm{t}, J=7.2 \mathrm{~Hz}, 2 \mathrm{H}), 7.15(\mathrm{t}, J$ $=8.1 \mathrm{~Hz}, 3 \mathrm{H}), 6.73-6.71(\mathrm{~m}, 3 \mathrm{H}), 2.17(\mathrm{~s}, 3 \mathrm{H})$.

${ }^{13} \mathrm{C}$ NMR $\left(150 \mathrm{MHz}\right.$, DMSO- $\left.d_{6}\right): \delta=172.8,146.4,141.3,129.3,128.4,125.3,123.2,119.2,111.8,22.2$. HRMS (ESI): $m / z[M+H]^{+}$calcd for $\mathrm{C}_{14} \mathrm{H}_{15} \mathrm{~N}_{2} \mathrm{O}^{+}: 227.1179$; found: 227.1180 .

\section{7) $N, N^{\prime}$-Diphenylpivalohydrazide (3as)}<smiles>CC(C)(C)C(=O)N(Nc1ccccc1)c1ccccc1</smiles>

Prepared according to the general procedure from azobenzene $1 \mathrm{a}(36.4 \mathrm{mg}, 0.2 \mathrm{mmol}$ ) and 3,3-dimethyl-2-oxobutanoic acid $2 s$ ( $26.0 \mathrm{mg}, 0.2 \mathrm{mmol}, 1$ equiv). Purified by column chromatography on silica gel (PE: EtOAc $=10: 1)$ to afford the pure product 3as.

White solid; yield: $20.9 \mathrm{mg}(39 \%)$, m.p. $122-144^{\circ} \mathrm{C}$.

${ }^{1} \mathrm{H}$ NMR $\left(600 \mathrm{MHz} \mathrm{CDCl}_{3}\right): \delta=7.39-7.37(\mathrm{~m}, 2 \mathrm{H}), 7.29(\mathrm{t}, J=8.1 \mathrm{~Hz}, 2 \mathrm{H}), 7.22-7.19(\mathrm{~m}, 2 \mathrm{H}), 7.17-7.15$ $(\mathrm{m}, 1 \mathrm{H}), 6.87(\mathrm{~s}, 1 \mathrm{H}), 6.83-6.81(\mathrm{~m}, 2 \mathrm{H}), 1.38(\mathrm{~s}, 9 \mathrm{H})$.

${ }^{13} \mathrm{C}$ NMR $\left(150 \mathrm{MHz}, \mathrm{CDCl}_{3}\right): \delta=180.6,145.7,142.4,129.0,128.6,126.4,125.1,120.9,113.9,40.4$, 27.9.

HRMS (ESI): $\mathrm{m} / \mathrm{z}[\mathrm{M}+\mathrm{H}]^{+}$calcd for $\mathrm{C}_{17} \mathrm{H}_{21} \mathrm{~N}_{2} \mathrm{O}^{+}:$269.1648; found:269.1649.

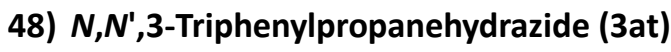<smiles>O=C(CCc1ccccc1)N(Nc1ccccc1)c1ccccc1</smiles> 
Prepared according to the general procedure from azobenzene 1a (36.4 mg, $0.2 \mathrm{mmol}$ ), 2-oxo-4-phenylbutanoic acid $\mathbf{2 t}$ (35.6 mg, $0.2 \mathrm{mmol}, 1$ equiv). Purified by column chromatography on silica gel (PE: EtOAc $=10: 1)$ to afford the pure product 3at.

White solid; yield: $56.9 \mathrm{mg}(90 \%) ;$ m.p. $151-152^{\circ} \mathrm{C}$.

${ }^{1} \mathrm{H}$ NMR $\left(600 \mathrm{MHz}\right.$, DMSO- $\left.d_{6}\right): \delta=9.00(\mathrm{~s}, 1 \mathrm{H}), 7.55(\mathrm{~d}, J=6.8 \mathrm{~Hz}, 2 \mathrm{H}), 7.30(\mathrm{t}, J=7.8 \mathrm{~Hz}, 2 \mathrm{H}), 7.24(\mathrm{t}, J=$ $7.2 \mathrm{~Hz}, 2 \mathrm{H}), 7.20-7.11(\mathrm{~m}, 6 \mathrm{H}), 6.73-6.68(\mathrm{~m}, 3 \mathrm{H}), 2.91-2.73(\mathrm{~m}, 4 \mathrm{H})$.

${ }^{13} \mathrm{C}$ NMR $\left(150 \mathrm{MHz}\right.$, DMSO- $\left.d_{6}\right): \delta=174.3,146.3,141.2,129.2,128.4,128.28,128.26,125.9,125.3$,

$123.3,119.2,111.9,35.2,30.5$.

HRMS (ESI): $m / z[M+H]^{+}$calcd for $\mathrm{C}_{21} \mathrm{H}_{21} \mathrm{~N}_{2} \mathrm{O}^{+}: 317.1648$; found: 317.1649 .

\section{References}

[1] Zhang, C.; Jiao, N. Angew. Chem. Int. Ed. 2010, 49, 6174.

[2] Lian, Y.; Bergman, R. G.; Lavis, L. D.; Ellman, J. A. J. Am. Chem. Soc. 2013, 135, 7122.

[3] Kuldeep, W.; Yang, C.; West, P. R.; Deming, K. C.; Chemburkar, S. R.; Reddy, R. E. Synth. Commun. 2008, 38, 4434.

[4] Kageyama, Y; Murata, S. J. Org. Chem. 2005, 70, 3140.

\section{Cope of NMR Spectra}


${ }^{1} \mathrm{H}$ NMR $\left(400 \mathrm{MHz}, \mathrm{CDCl}_{3}\right)$<smiles>O=C(c1ccccc1)N(Nc1ccccc1)c1ccccc1</smiles>

3aa

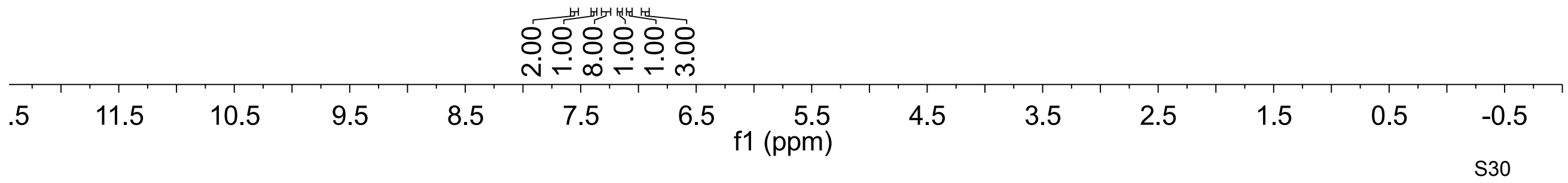




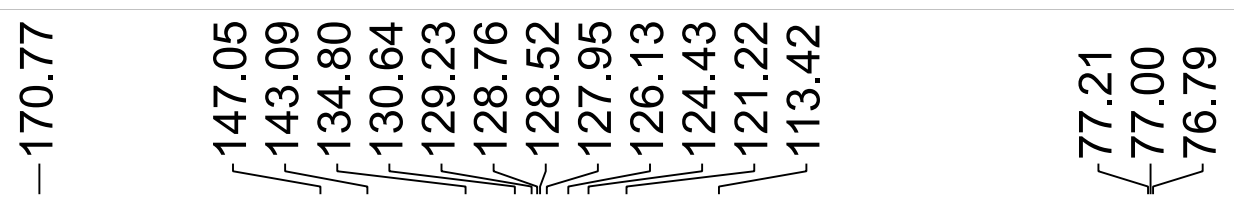

${ }^{13} \mathrm{C}$ NMR $\left(150 \mathrm{MHz}, \mathrm{CDCl}_{3}\right)$<smiles>CCCCCC(=O)N(Nc1ccccc1)c1ccccc1</smiles>

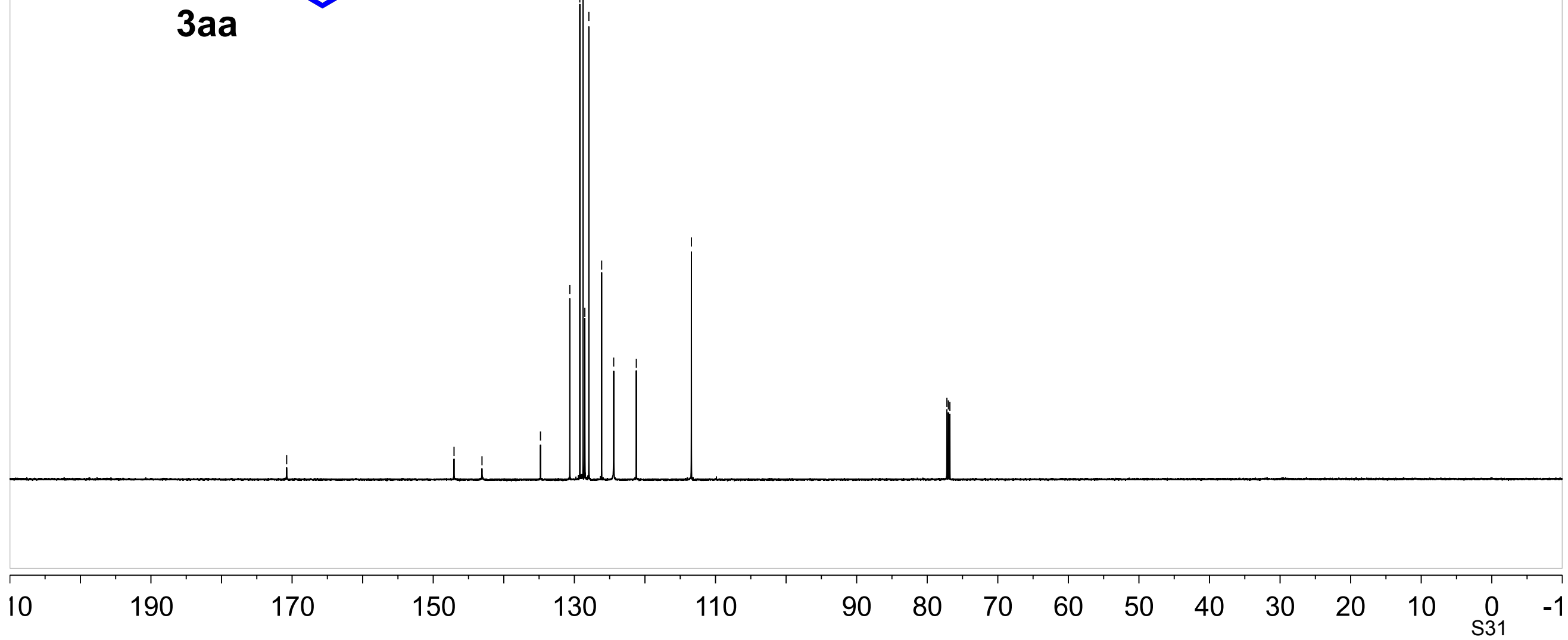




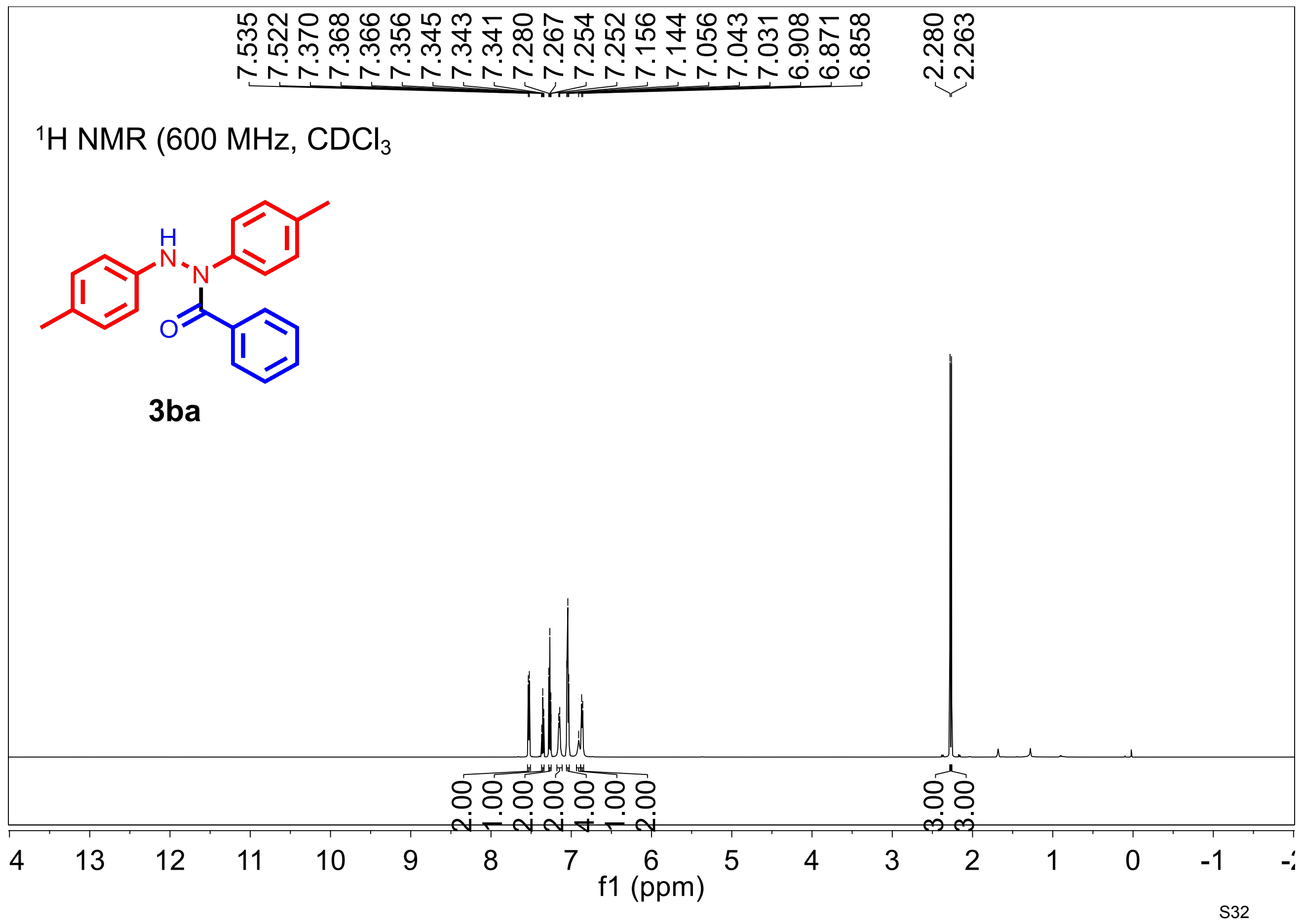




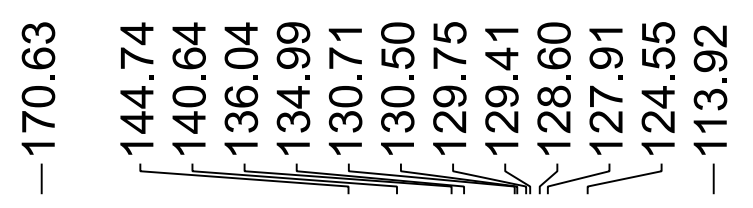

กำ

Nペ

赵

○ं

N

${ }^{13} \mathrm{C}$ NMR (150 MHz, $\left.\mathrm{CDCl}_{3}\right)$

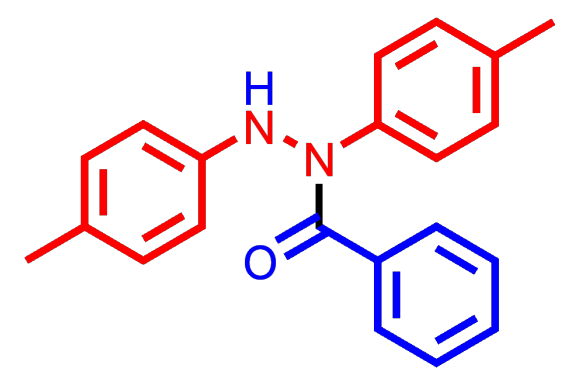

$3 b a$ 


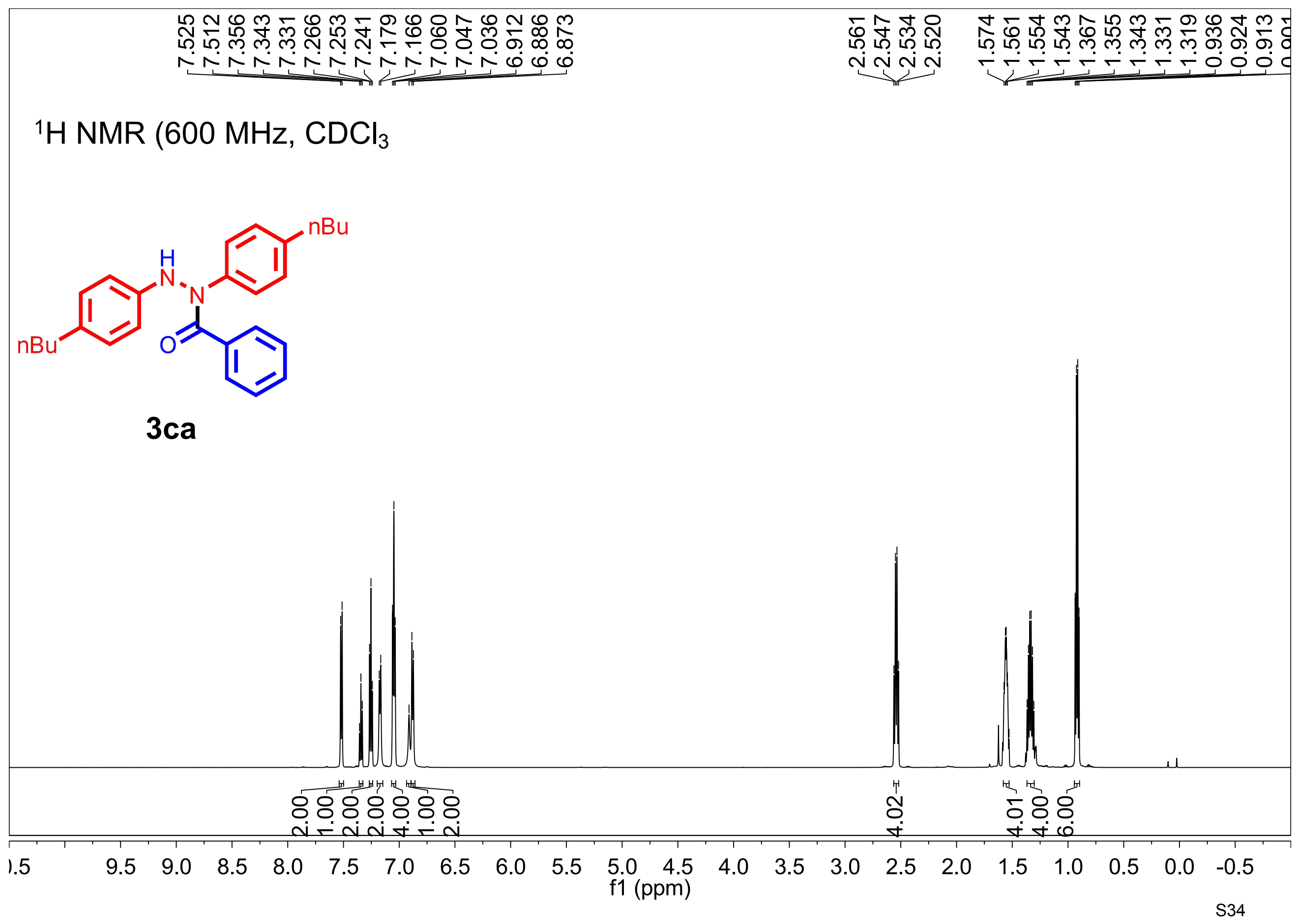




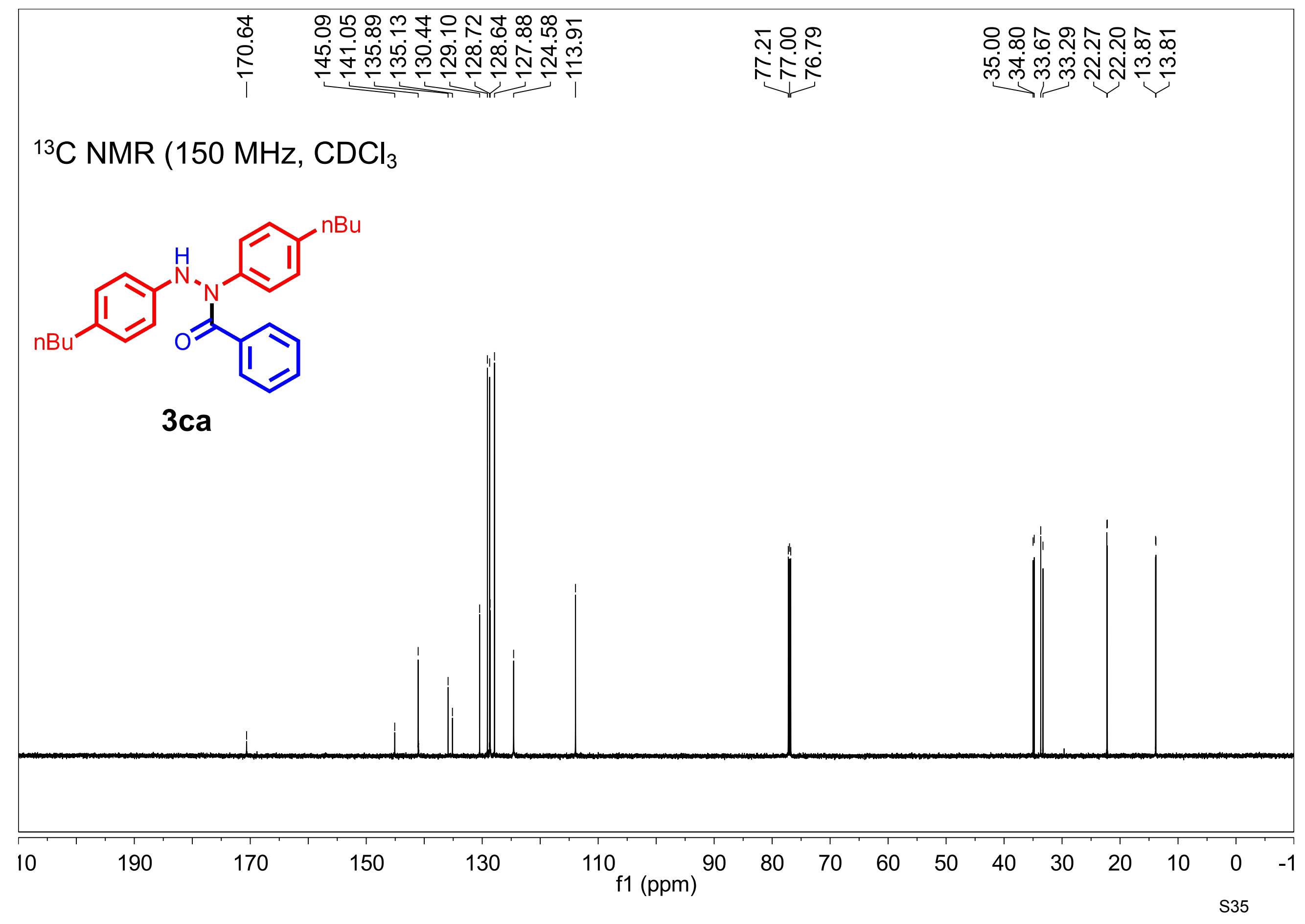


${ }^{1} \mathrm{H}$ NMR $\left(600 \mathrm{MHz}, \mathrm{CDCl}_{3}\right)$<smiles>CC(C)(C)c1ccc(NN(C(=O)c2ccccc2)c2ccc(C(C)(C)C)cc2)cc1</smiles>

3da

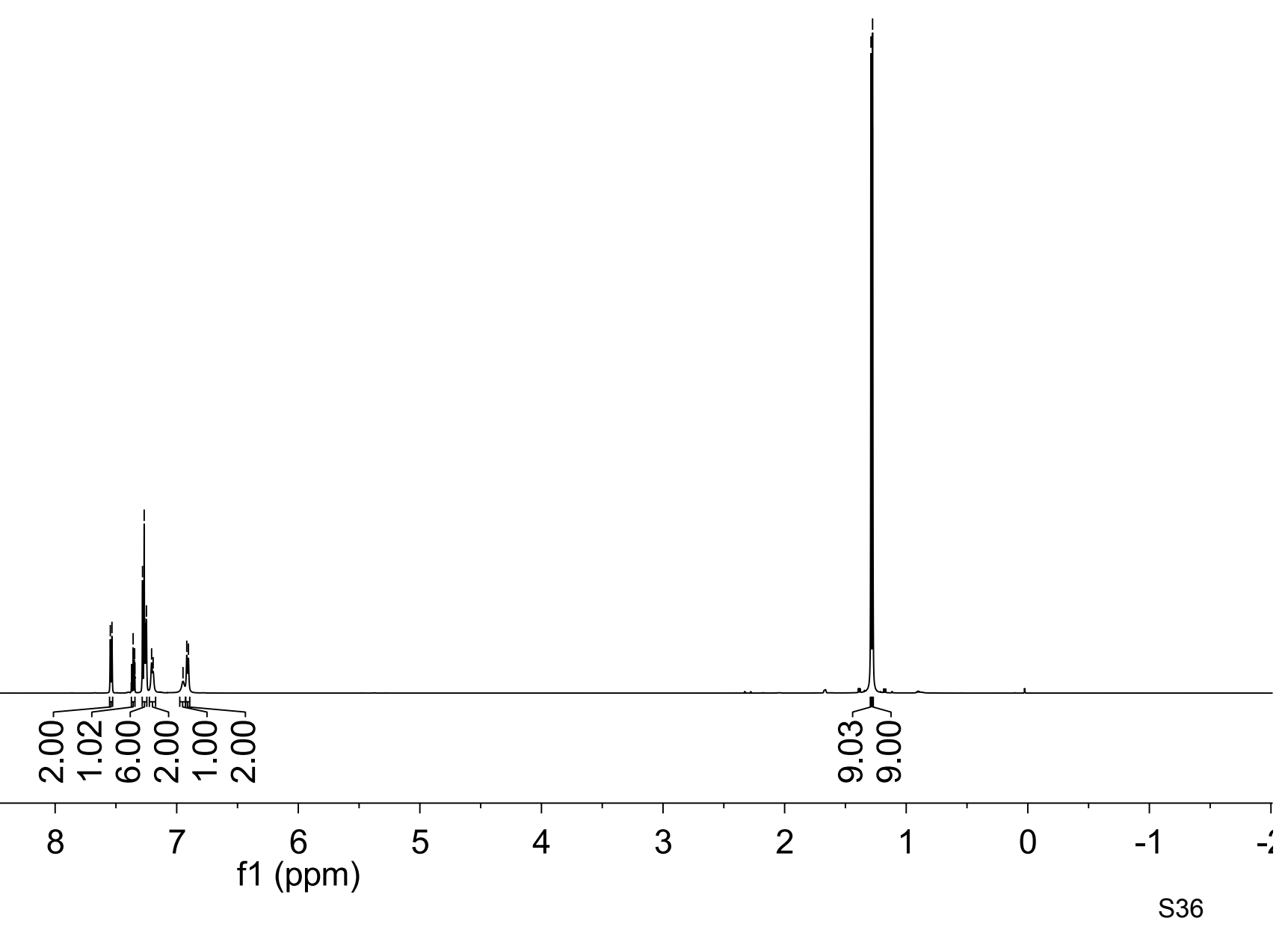




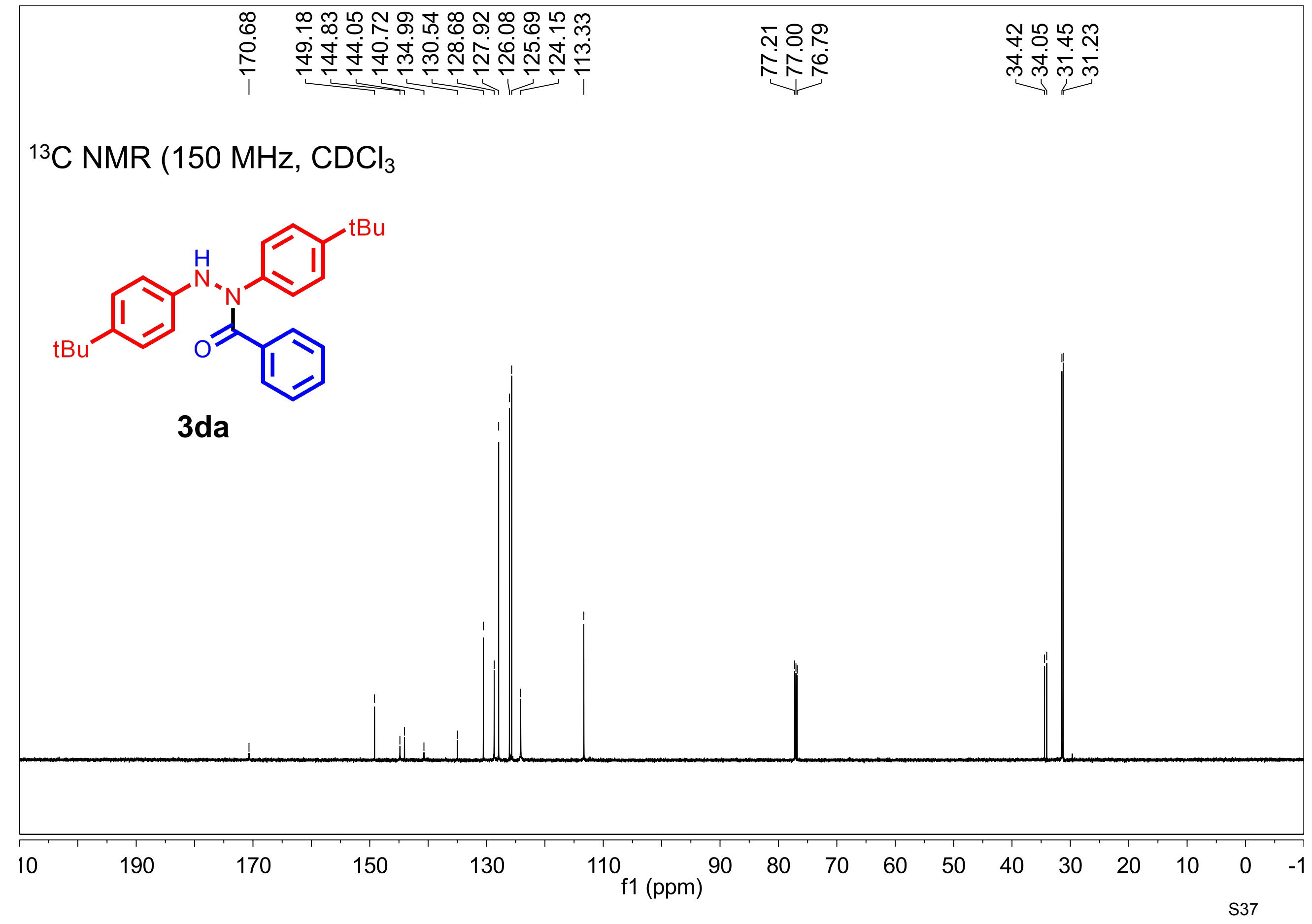




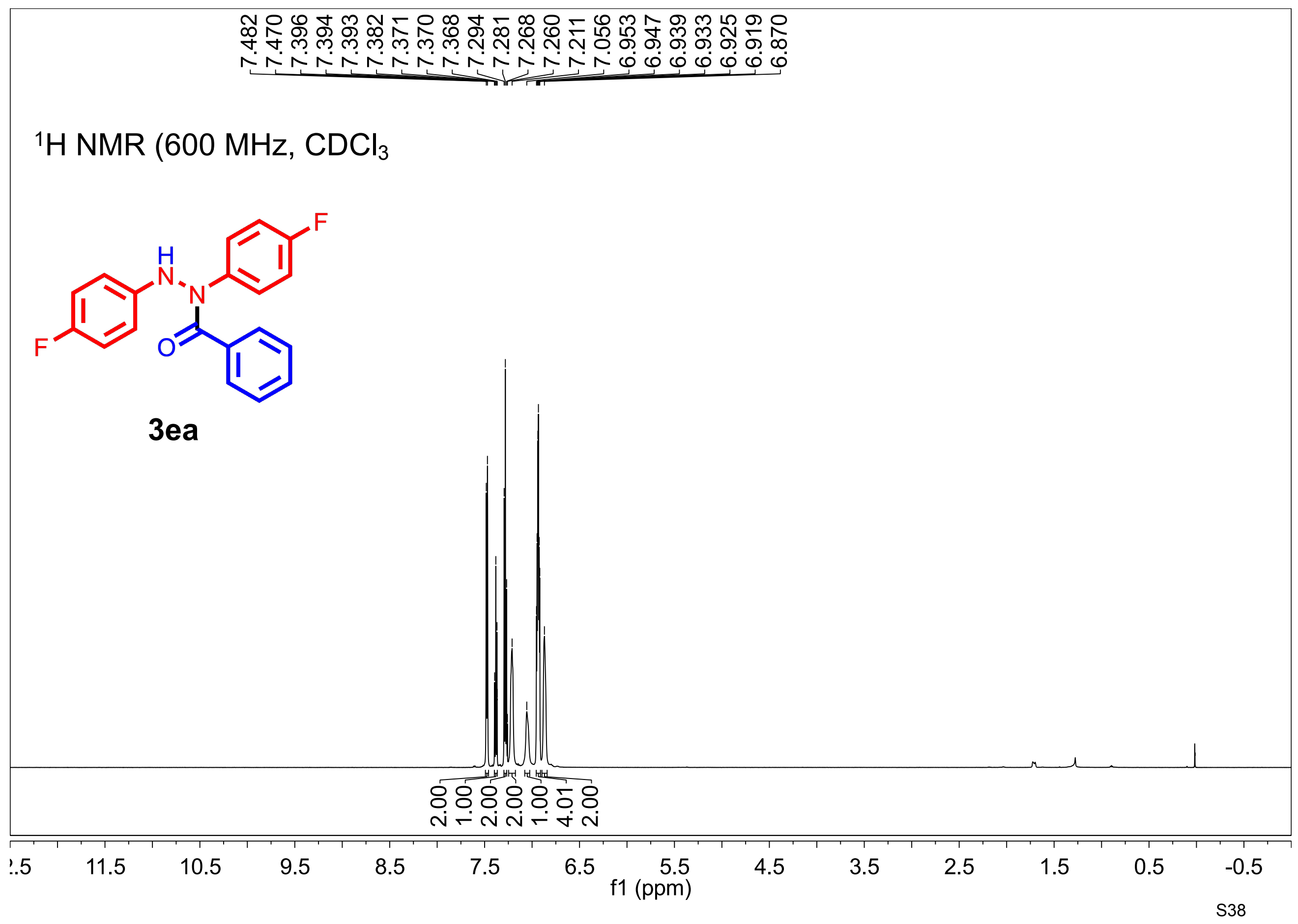




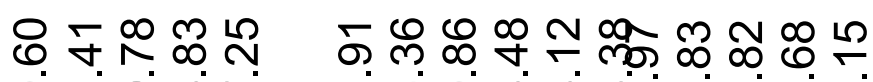

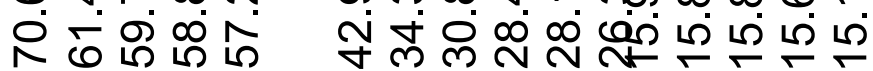

กุำ

Nลำ

।

岱西

${ }^{13} \mathrm{C}$ NMR $\left(150 \mathrm{MHz}, \mathrm{CDCl}_{3}\right)$<smiles>O=C(c1ccccc1)N(Nc1ccc(F)cc1)c1ccc(F)cc1</smiles>

3ea 
${ }^{19} \mathrm{~F}$ NMR $\left(287 \mathrm{MHz}, \mathrm{CDCl}_{3}\right)$

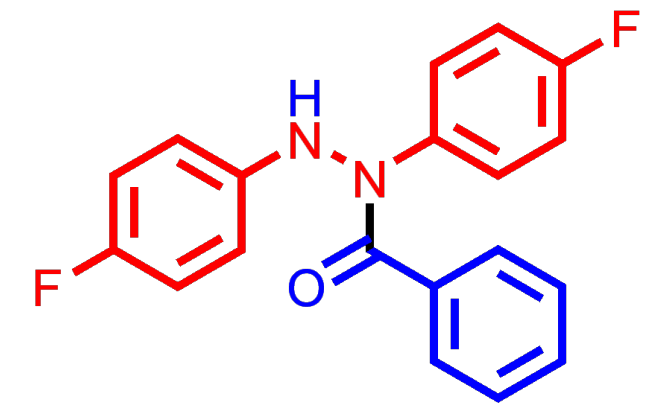

3ea

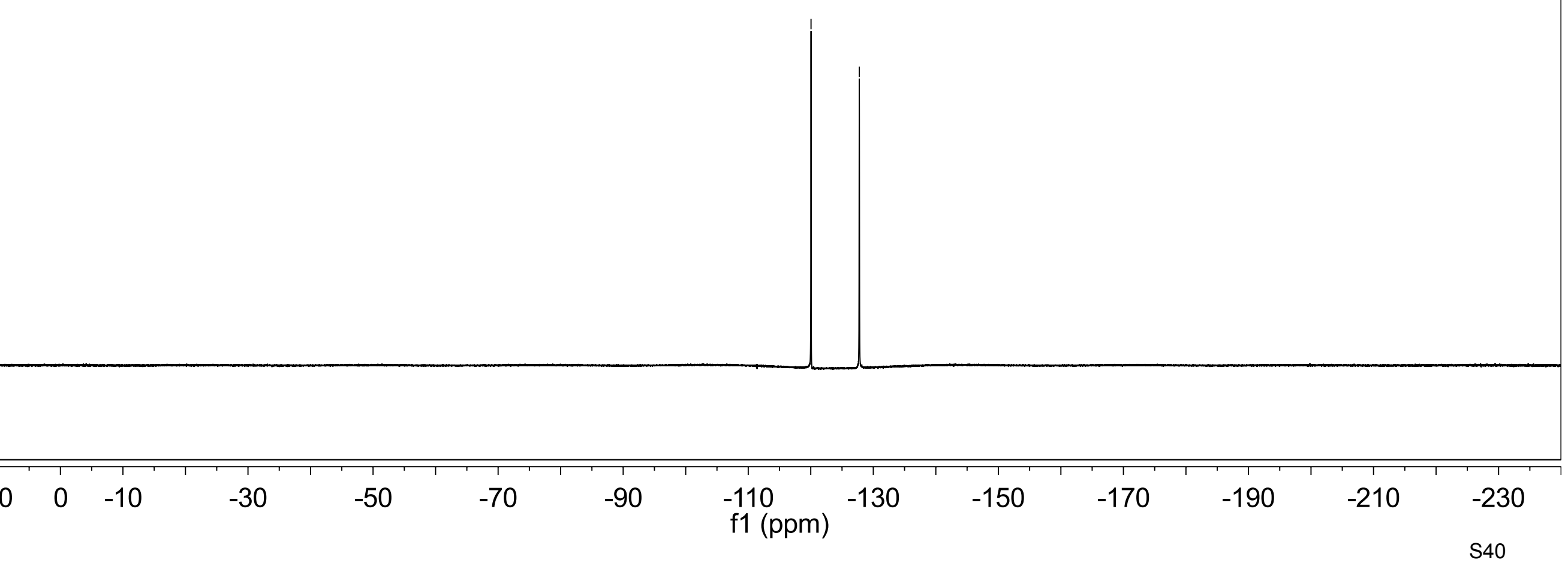


${ }^{1} \mathrm{H}$ NMR $\left(600 \mathrm{MHz}, \mathrm{CDCl}_{3}\right)$

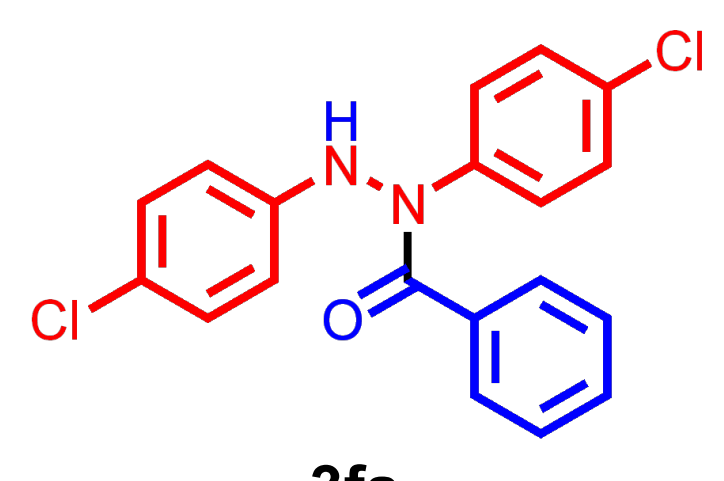

$3 f a$

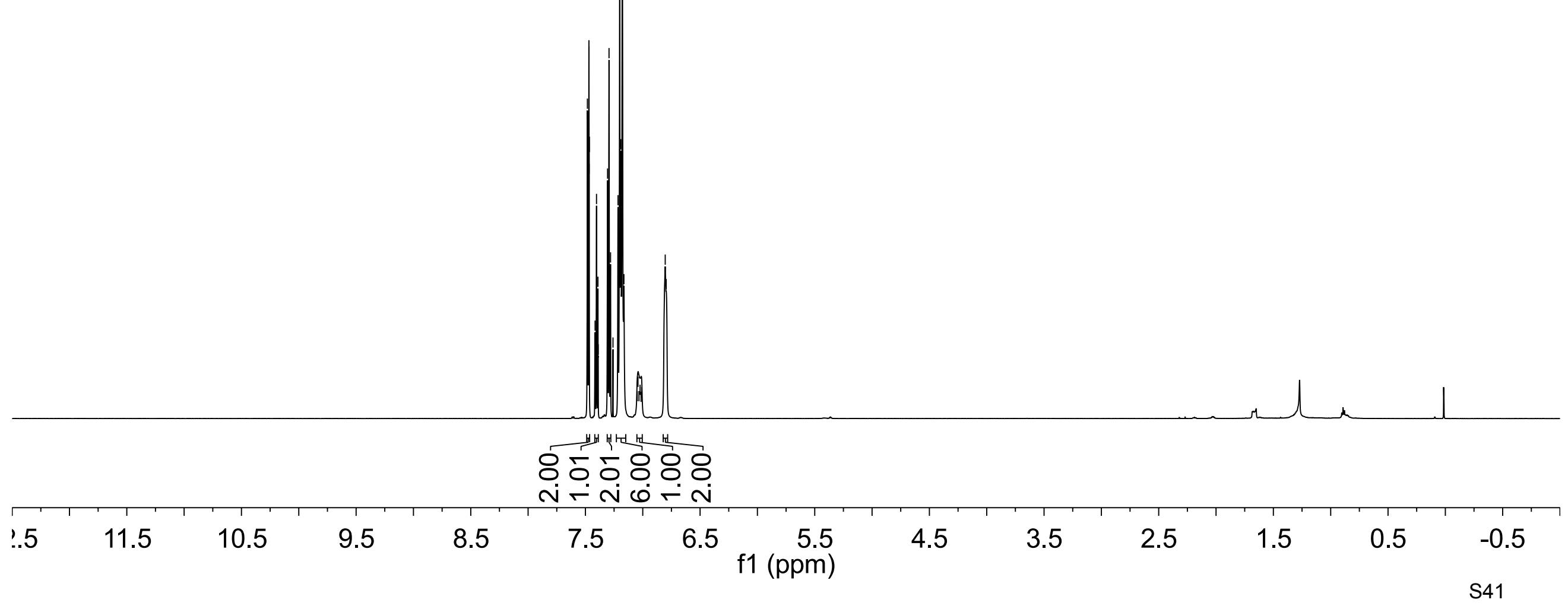




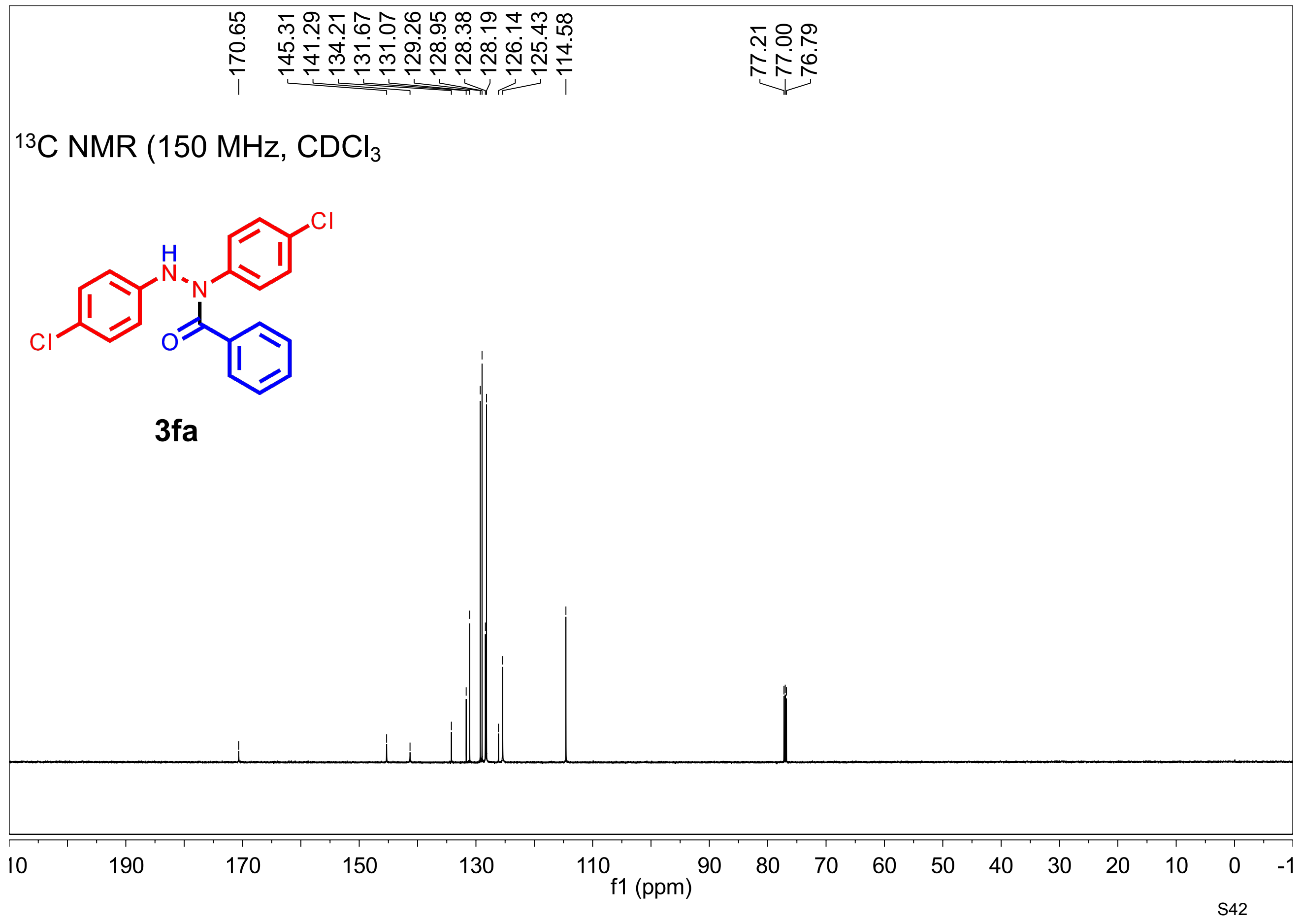




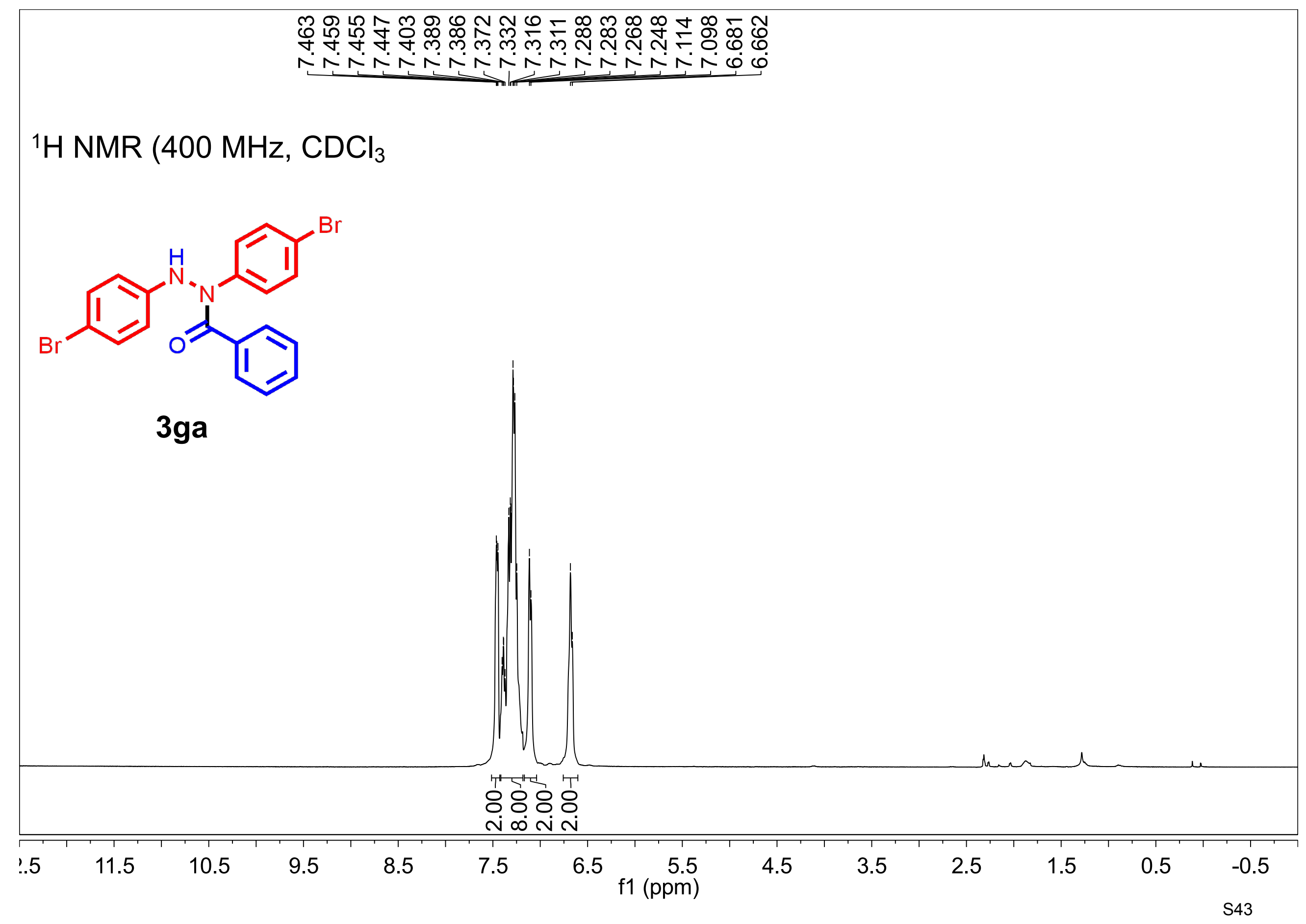




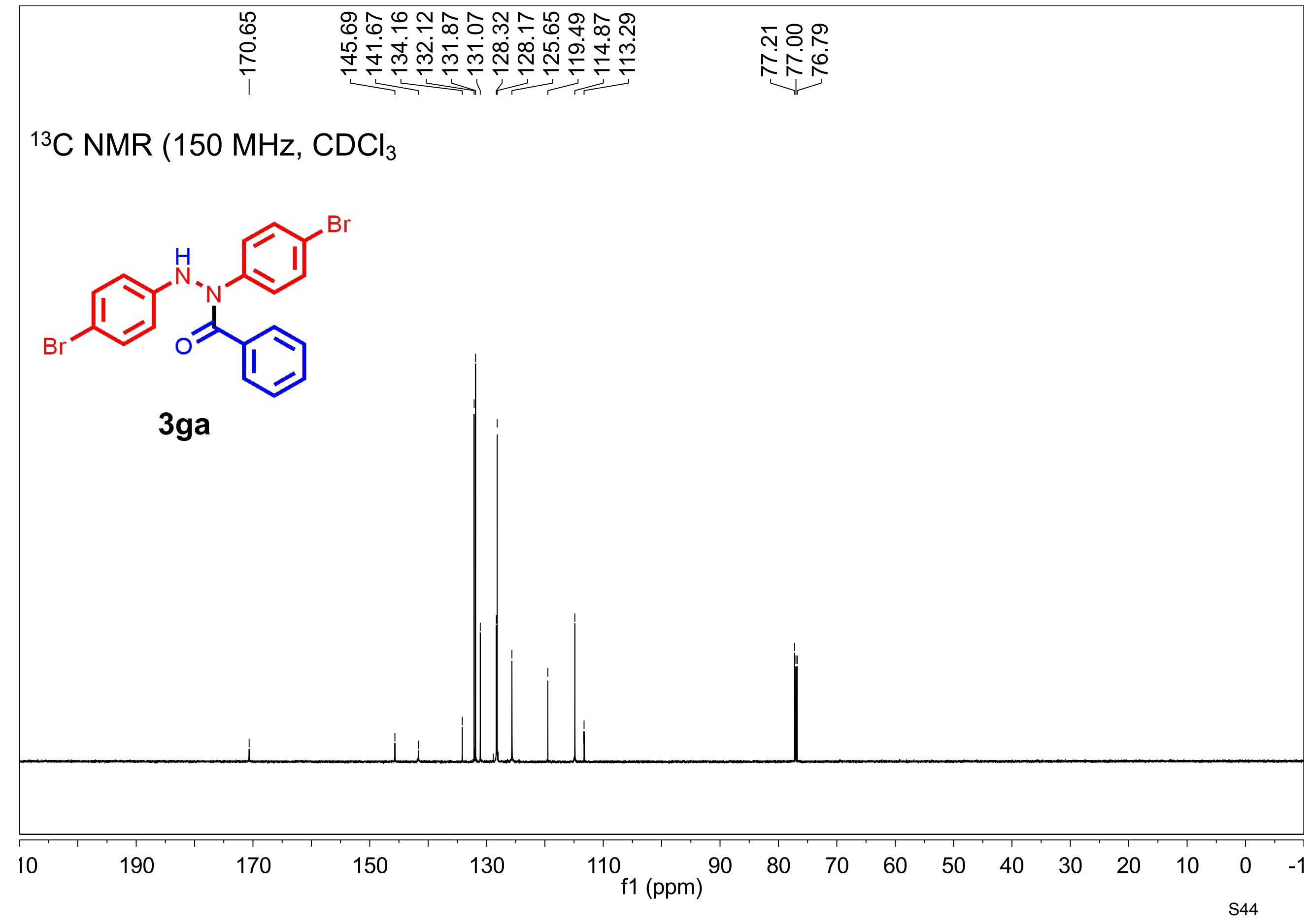




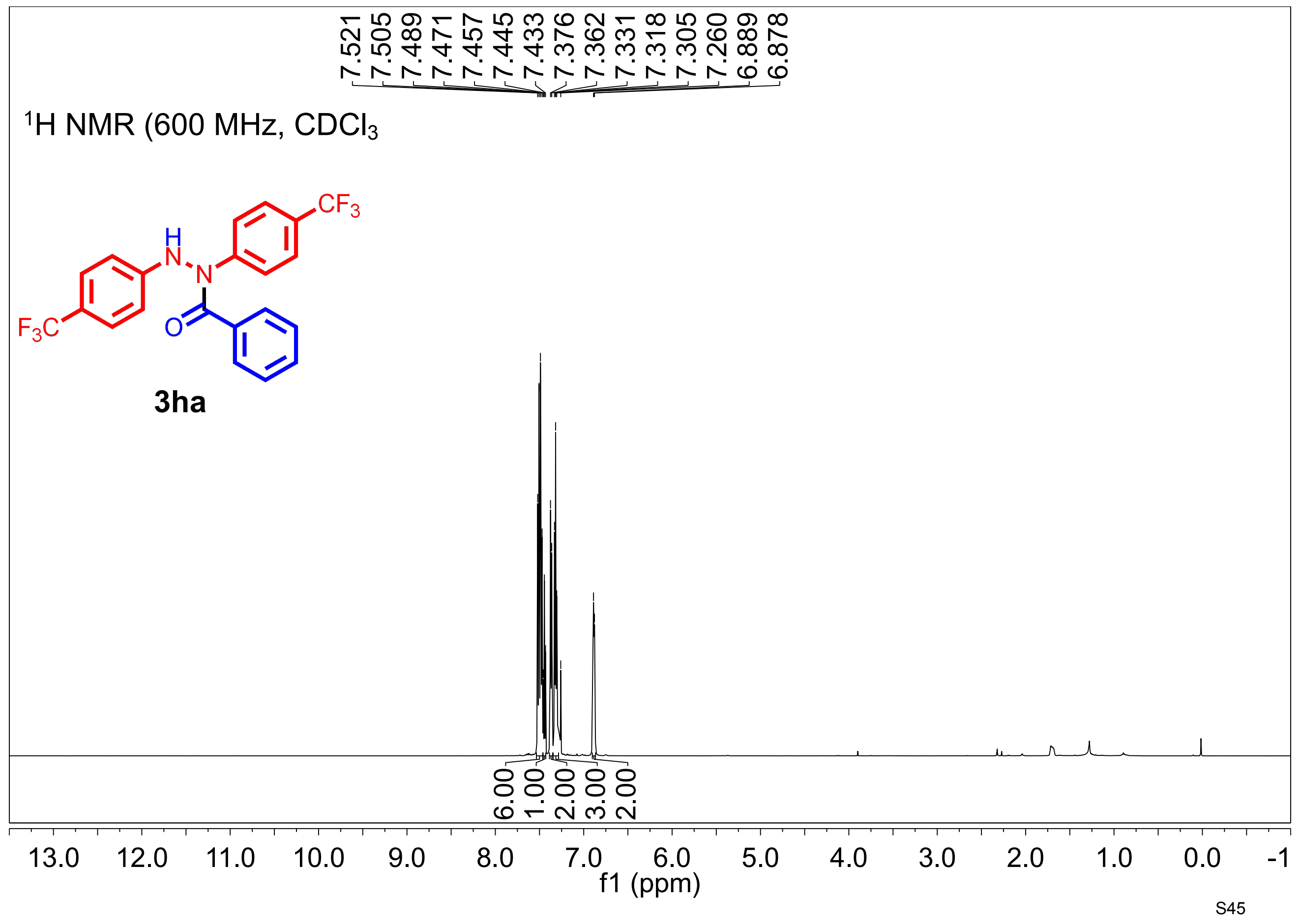




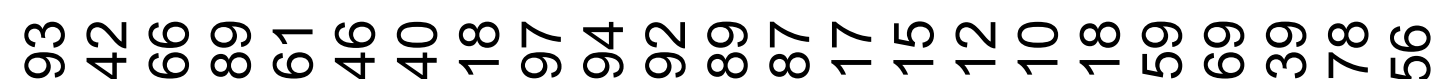

จ

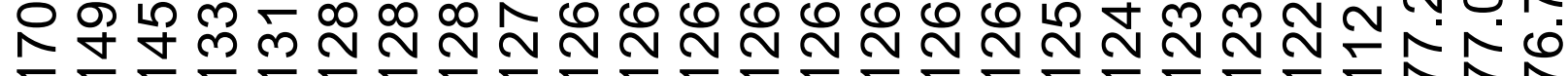

${ }^{13} \mathrm{C}$ NMR (150 $\left.\mathrm{MHz}, \mathrm{CDCl}_{3}\right)$

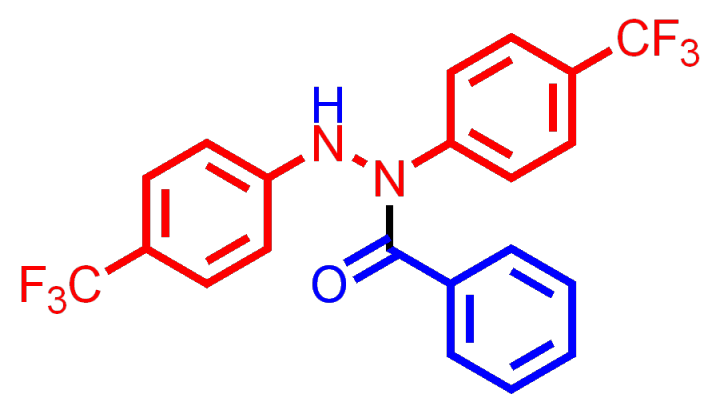

3ha

\begin{tabular}{|c|c|c|c|c|c|c|c|c|c|c|c|c|}
\hline 230 & 210 & 190 & 170 & 150 & 130 & $\begin{array}{c}110 \\
\mathrm{f} 1(\mathrm{ppm})\end{array}$ & 90 & 70 & 50 & 30 & 10 & $\begin{array}{l}-10 \\
46\end{array}$ \\
\hline
\end{tabular}


웅 웅

ڤิن

${ }^{19} \mathrm{~F}$ NMR (287 $\left.\mathrm{MHz} \mathrm{CDCl}_{3}\right)$

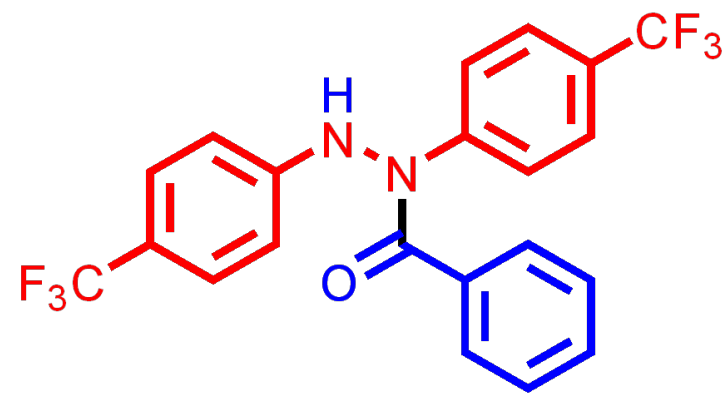

3ha 
${ }^{1} \mathrm{H}$ NMR $\left(600 \mathrm{MHz}, \mathrm{CDCl}_{3}\right)$

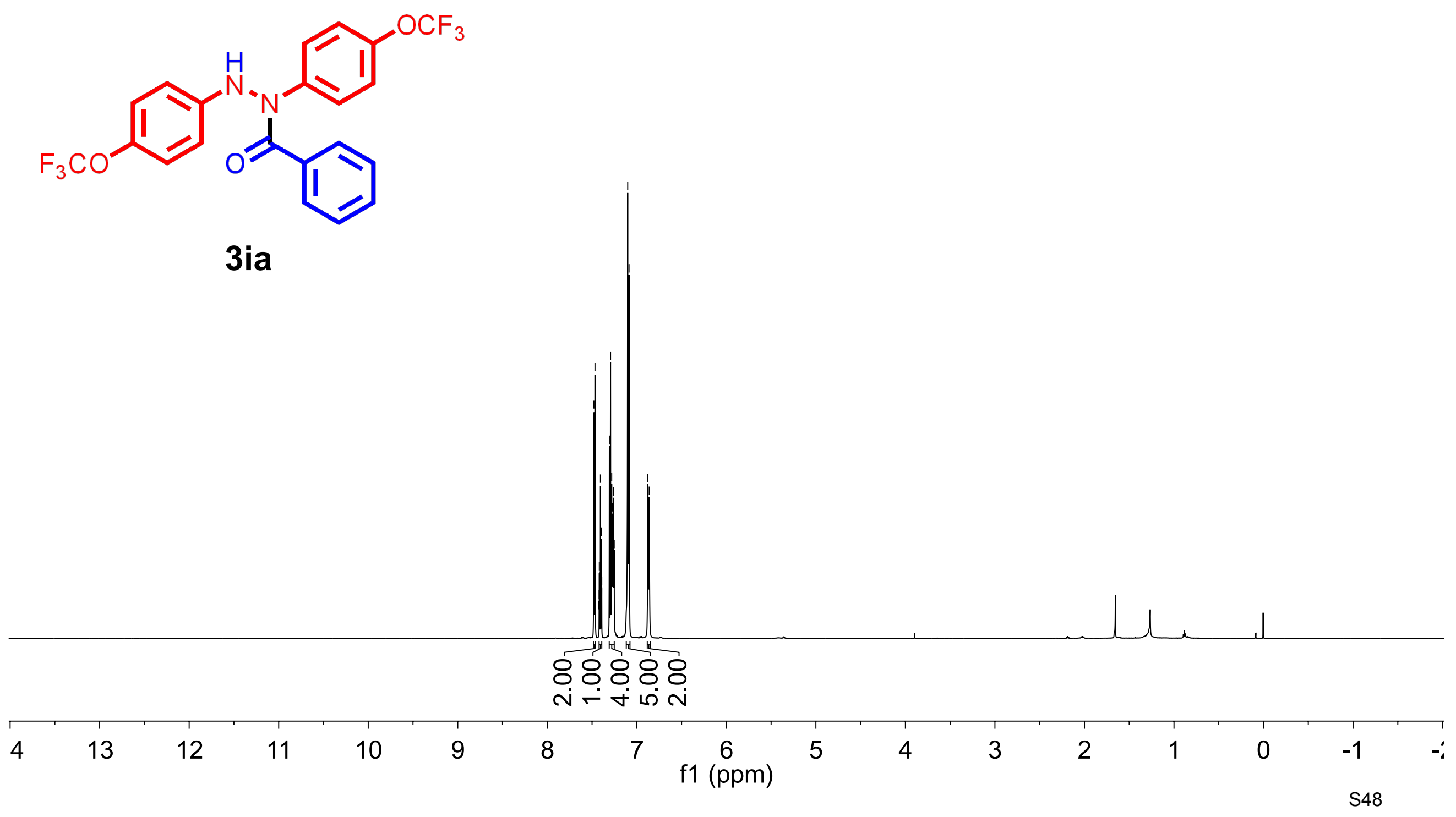




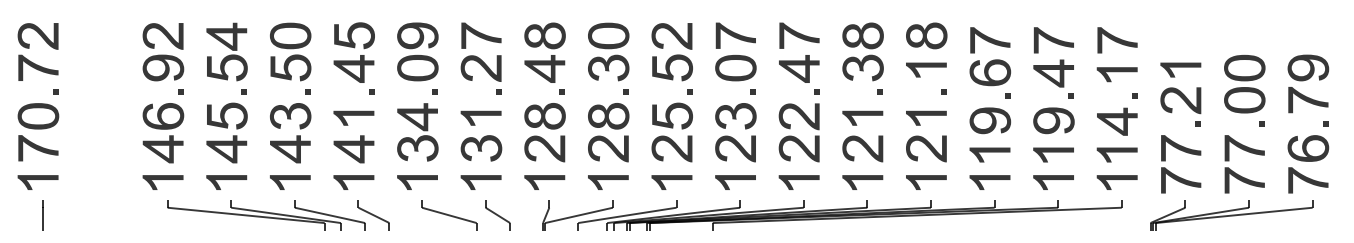

${ }^{13} \mathrm{C}$ NMR $\left(150 \mathrm{MHz}, \mathrm{CDCl}_{3}\right)$

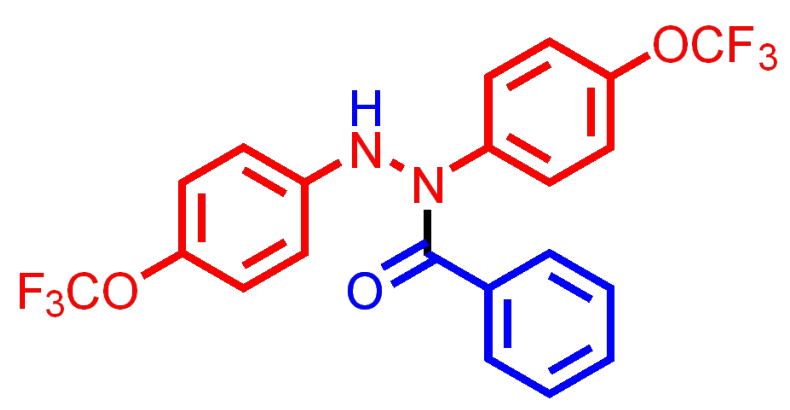

3 ia

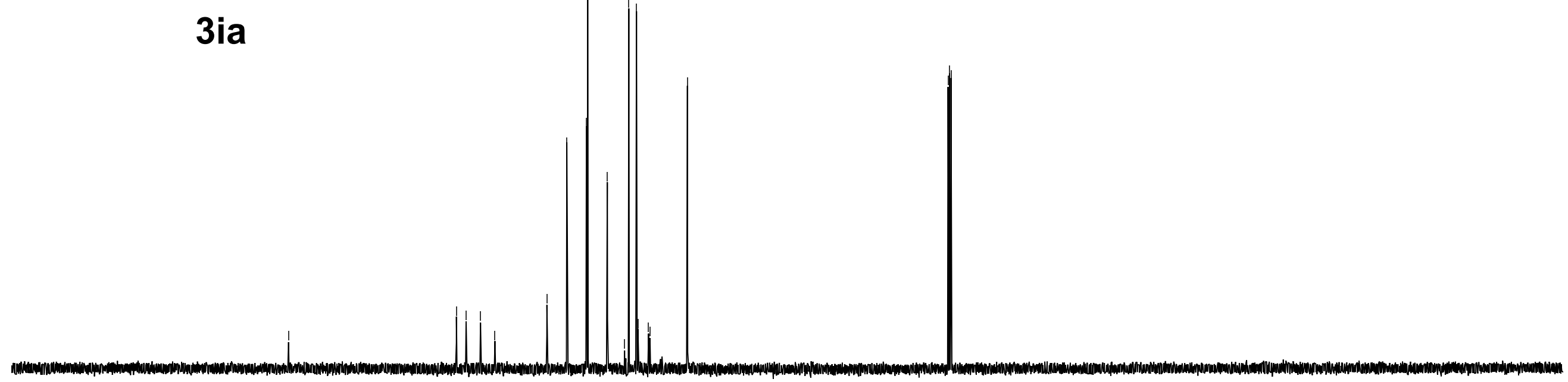

$10200190180170160150140130120 \begin{gathered}110 \quad \begin{array}{l}100 \\ \mathrm{f} 1(\mathrm{ppm})\end{array} \\ 00\end{gathered}$ 
${ }^{19} \mathrm{~F}$ NMR $\left(287 \mathrm{MHz}, \mathrm{CDCl}_{3}\right)$<smiles>O=C(c1ccccc1)N(Nc1ccc(OC(F)(F)F)cc1)c1ccc(OC(F)(F)F)cc1</smiles>

3ia

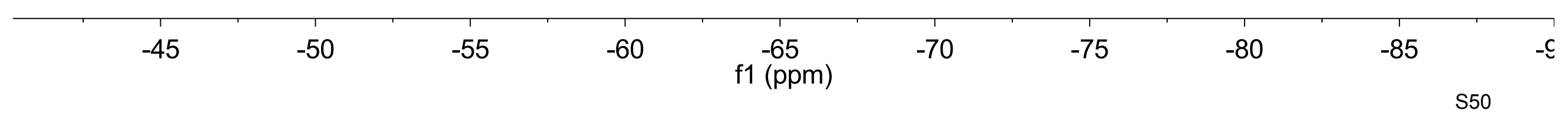


${ }^{1} \mathrm{H}$ NMR $\left(600 \mathrm{MHz}, \mathrm{CDCl}_{3}\right)$

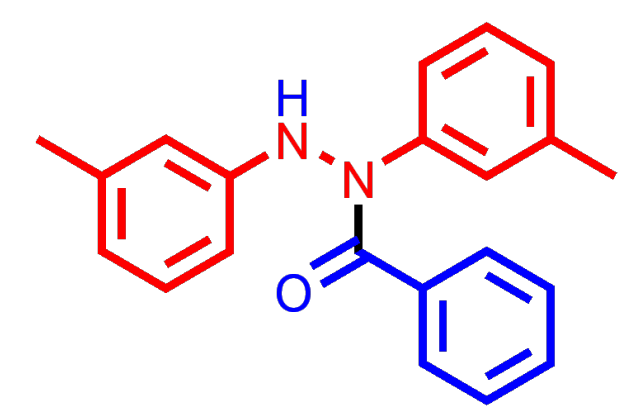

3ja

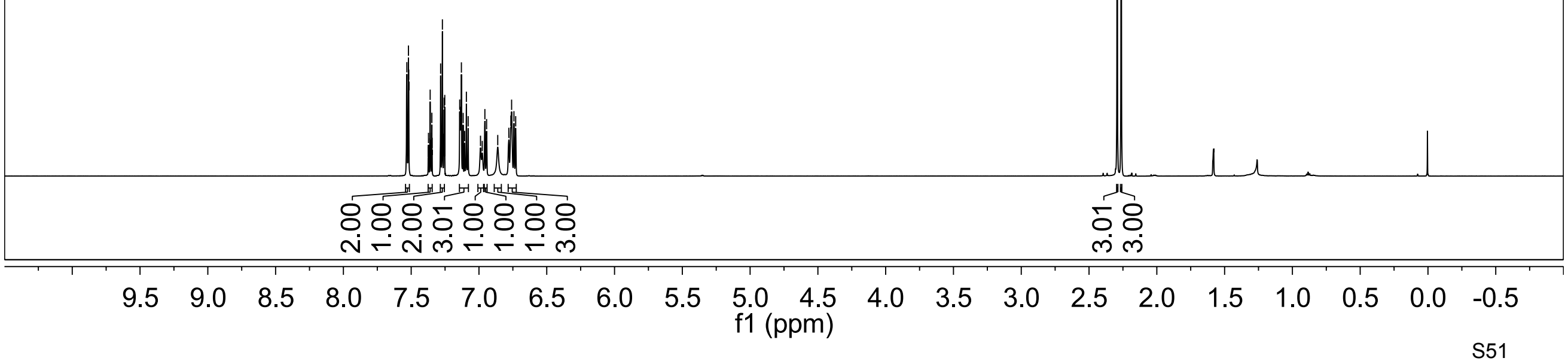


${ }^{13} \mathrm{C}$ NMR $\left(150 \mathrm{MHz}, \mathrm{CDCl}_{3}\right)$<smiles>Cc1cccc(NN(C(=O)c2ccccc2)c2cccc(C)c2)c1</smiles>

3ja

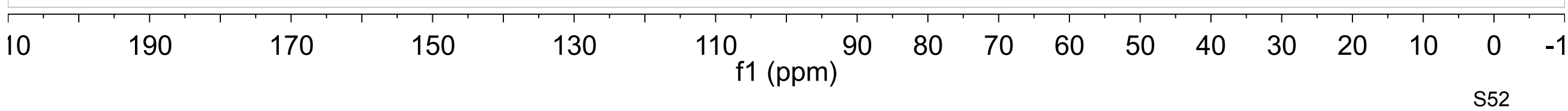




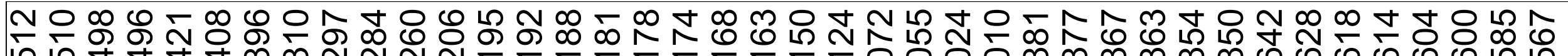
एँ

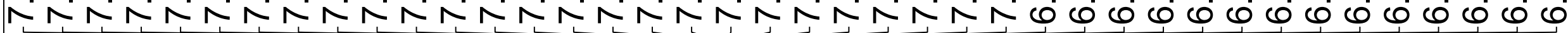

${ }^{1} \mathrm{H}$ NMR $\left(400 \mathrm{MHz}, \mathrm{CDCl}_{3}\right)$<smiles>O=C(c1ccccc1)N(Nc1cccc(F)c1)c1cccc(F)c1</smiles>

3ka

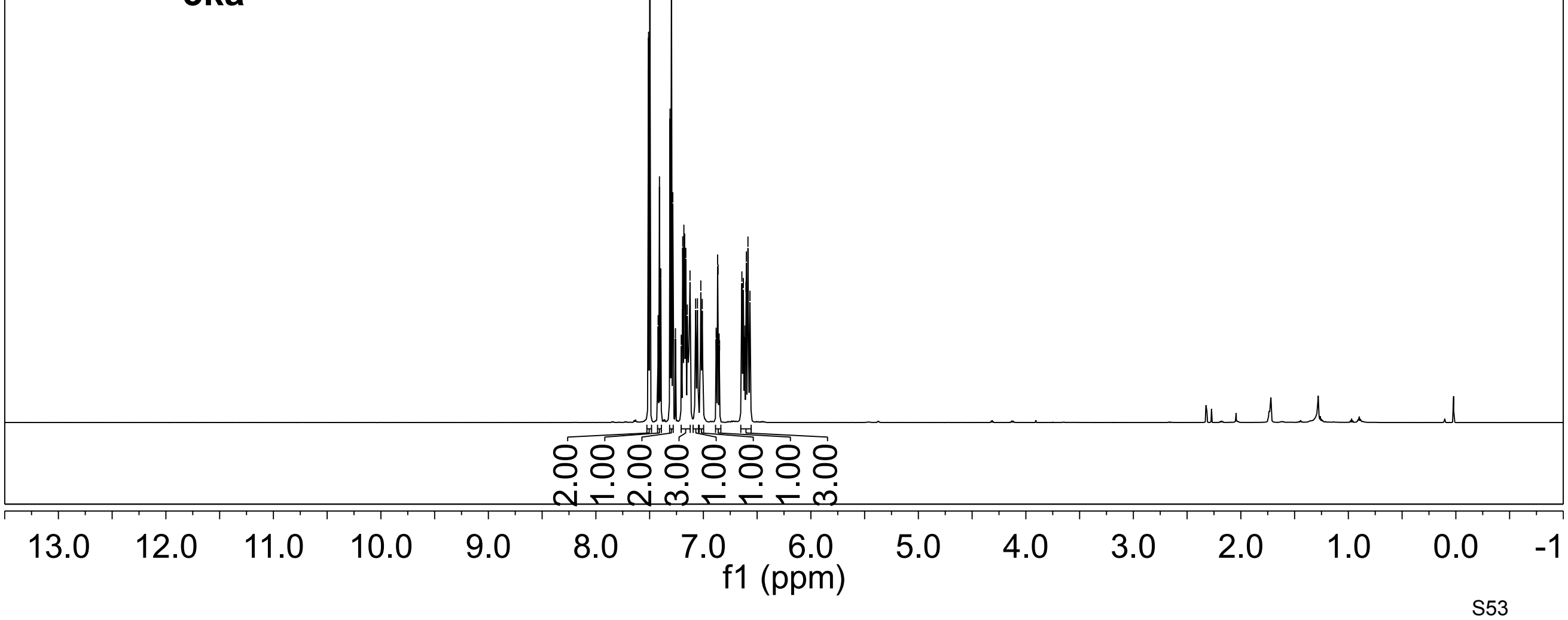




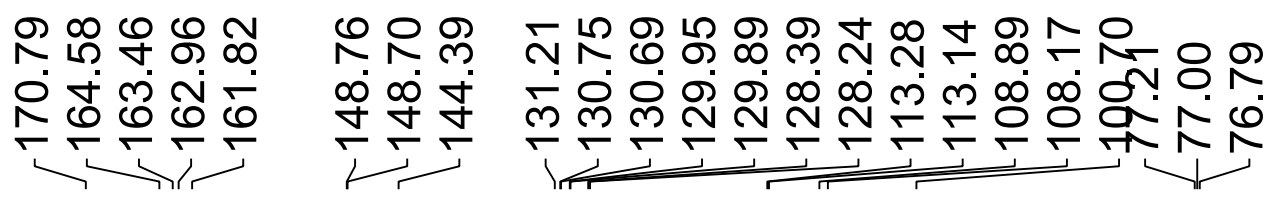

${ }^{13} \mathrm{C}$ NMR (150 MHz, $\left.\mathrm{CDCl}_{3}\right)$

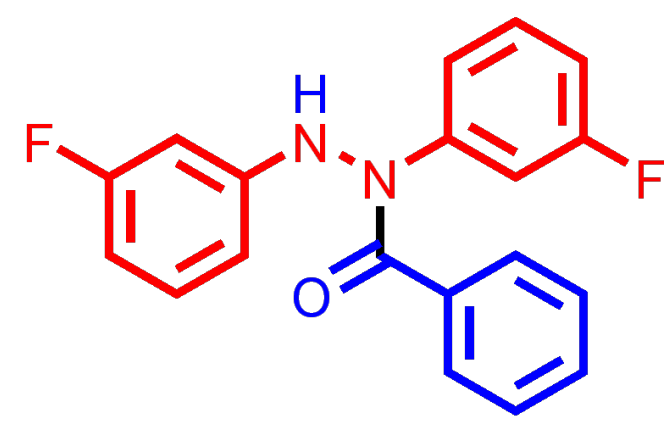

3ka

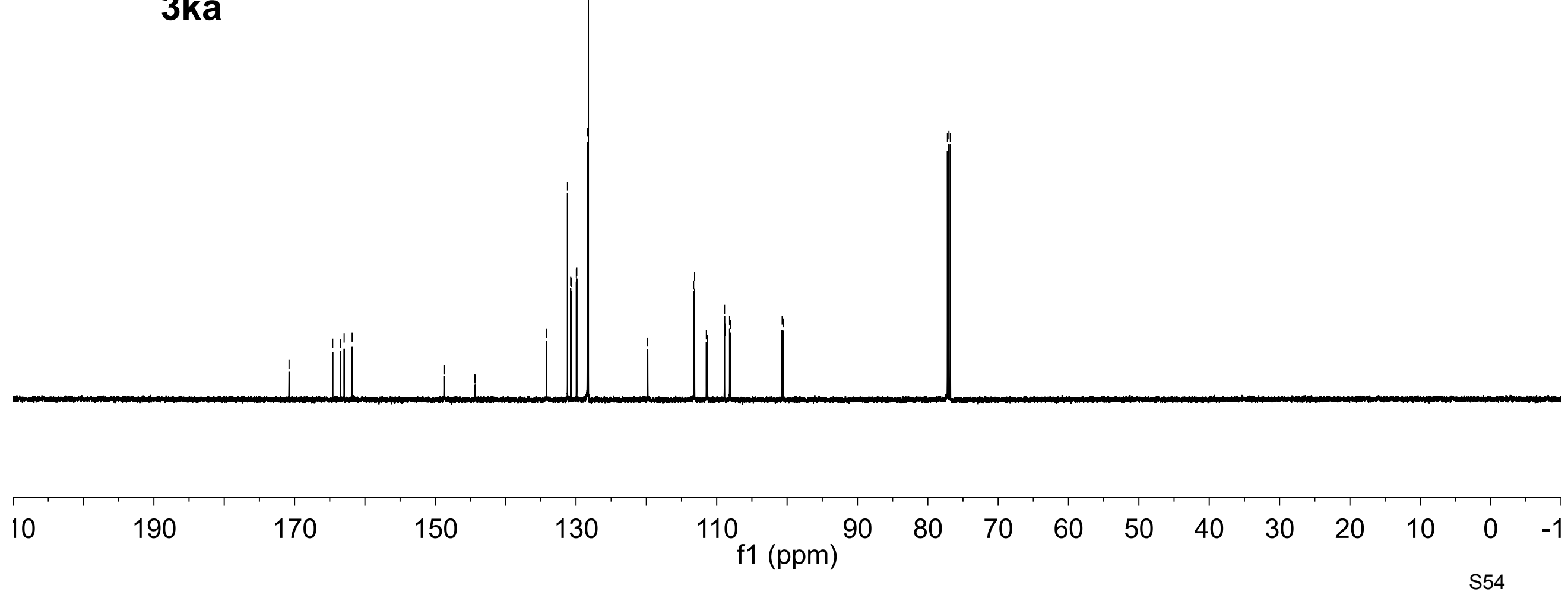


${ }^{19} \mathrm{~F}$ NMR (287 MHz, $\mathrm{CDCl}_{3}$ )

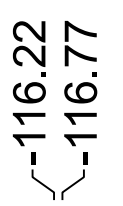

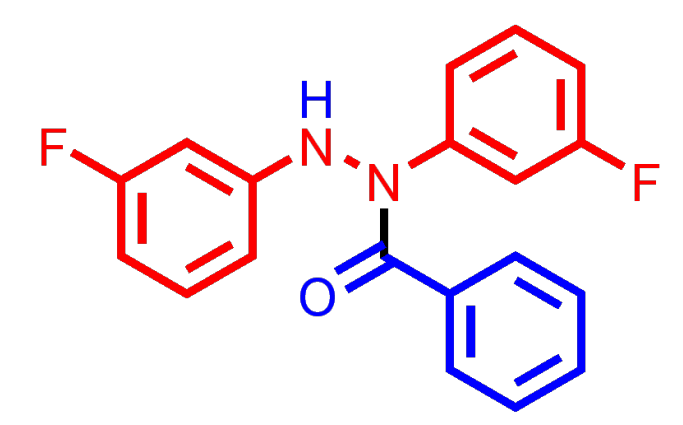

3ka

\begin{tabular}{|c|c|c|c|c|c|c|c|c|c|c|c|c|c|c|c|c|c|}
\hline 10 & -50 & -60 & -70 & -80 & -90 & -100 & -110 & $\begin{array}{c}-120 \\
\quad f 1(k)\end{array}$ & $\begin{array}{l}-130 \\
p m)\end{array}$ & -140 & -150 & -160 & -170 & -180 & -190 & -200 & -2 \\
\hline
\end{tabular}




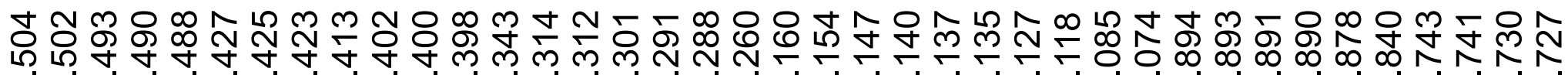

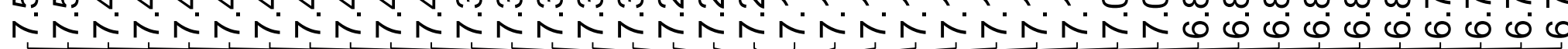

\section{${ }^{1} \mathrm{H}$ NMR $\left(600 \mathrm{MHz}, \mathrm{CDCl}_{3}\right)$}

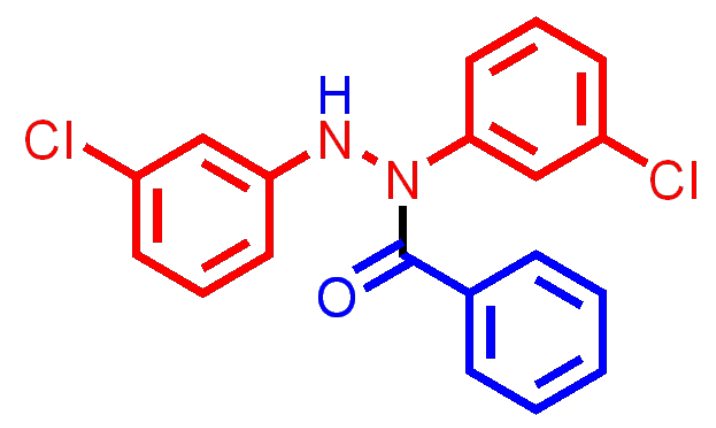

\section{3la}

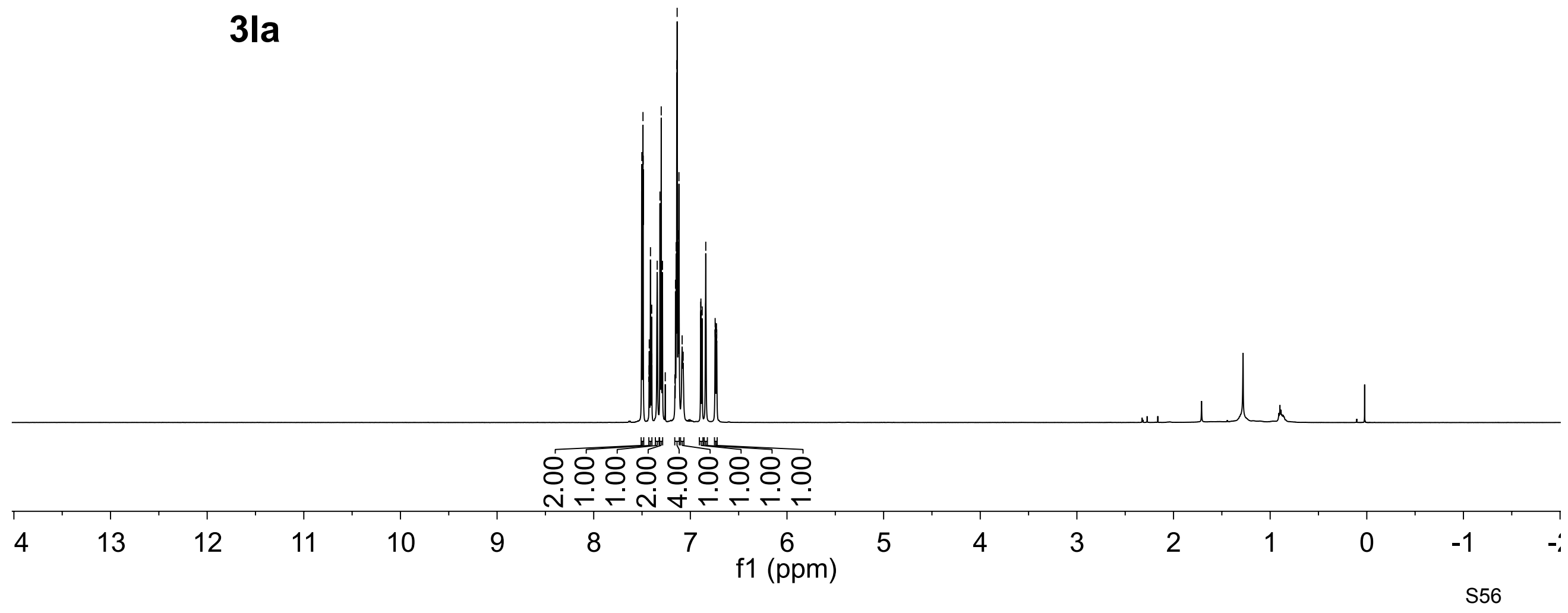




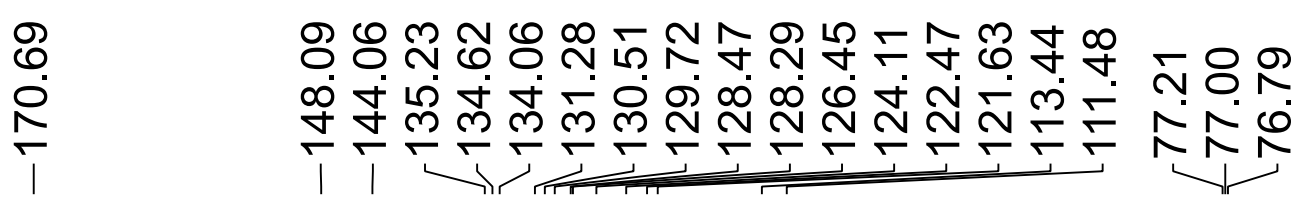

${ }^{13} \mathrm{C}$ NMR $\left(150 \mathrm{MHz}, \mathrm{CDCl}_{3}\right)$

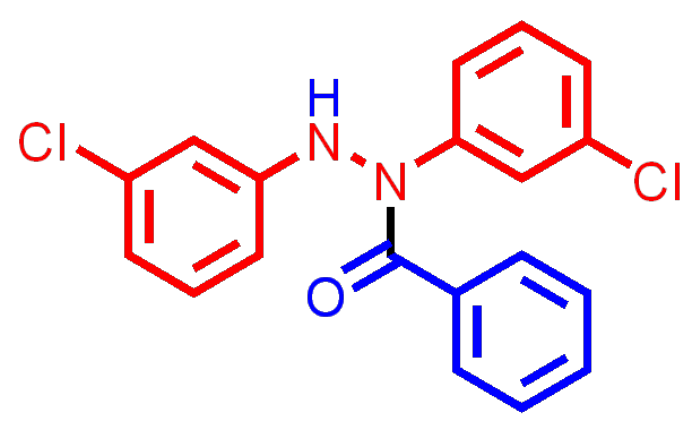

3la

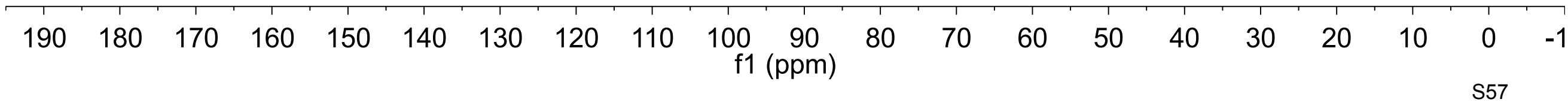


œ オ

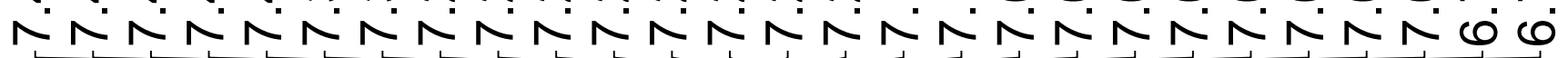

${ }^{1} \mathrm{H}$ NMR $\left(600 \mathrm{MHz}, \mathrm{CDCl}_{3}\right)$

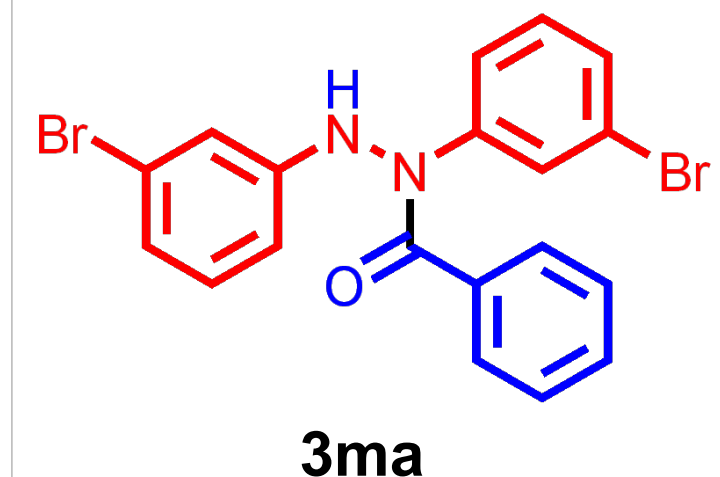

$88.0+1008$

लंलेलि-

\begin{tabular}{|c|c|c|c|c|c|c|c|c|c|c|c|c|}
\hline i. 12.0 & 11.0 & 10.0 & 9.0 & 8.0 & 7.0 & $\begin{array}{l}6.0 \\
\mathrm{f} 1(\mathrm{ppm})\end{array}$ & 5.0 & 4.0 & 3.0 & 2.0 & 1.0 & $\begin{array}{ll}0.0 & -1 \\
\text { s58 } & \end{array}$ \\
\hline
\end{tabular}




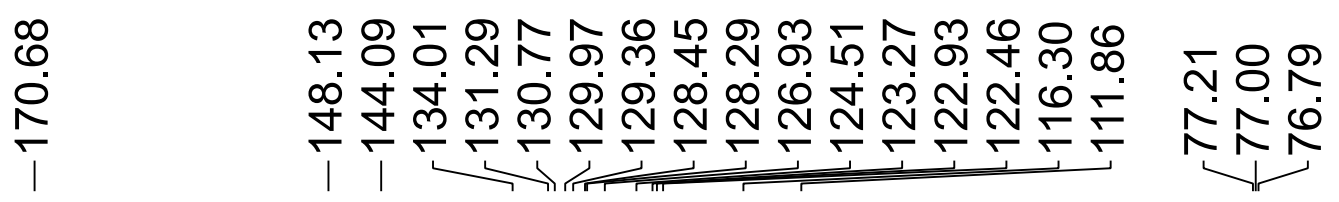

${ }^{13} \mathrm{C}$ NMR $\left(150 \mathrm{MHz}, \mathrm{CDCl}_{3}\right)$

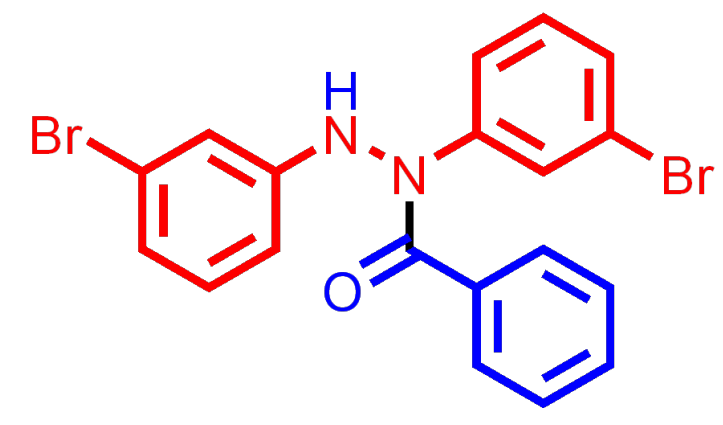

$3 \mathrm{ma}$

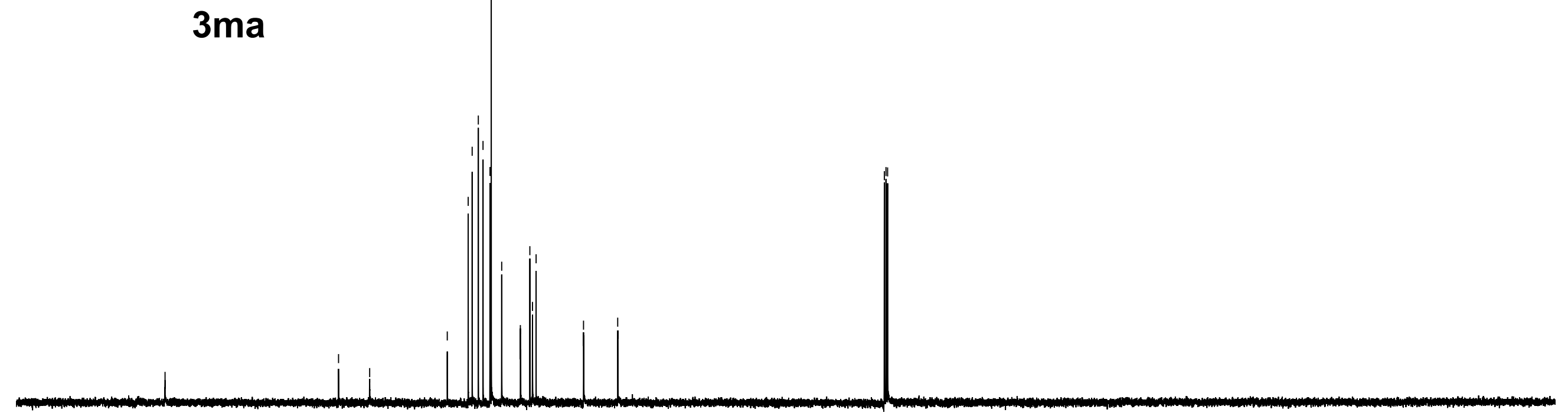

$\begin{array}{llll}70 & 60 \quad 50\end{array}$

$\begin{array}{lll}10 & 30 & 20\end{array}$


${ }^{1} \mathrm{H}$ NMR $\left(600 \mathrm{MHz}, \mathrm{CDCl}_{3}\right)$

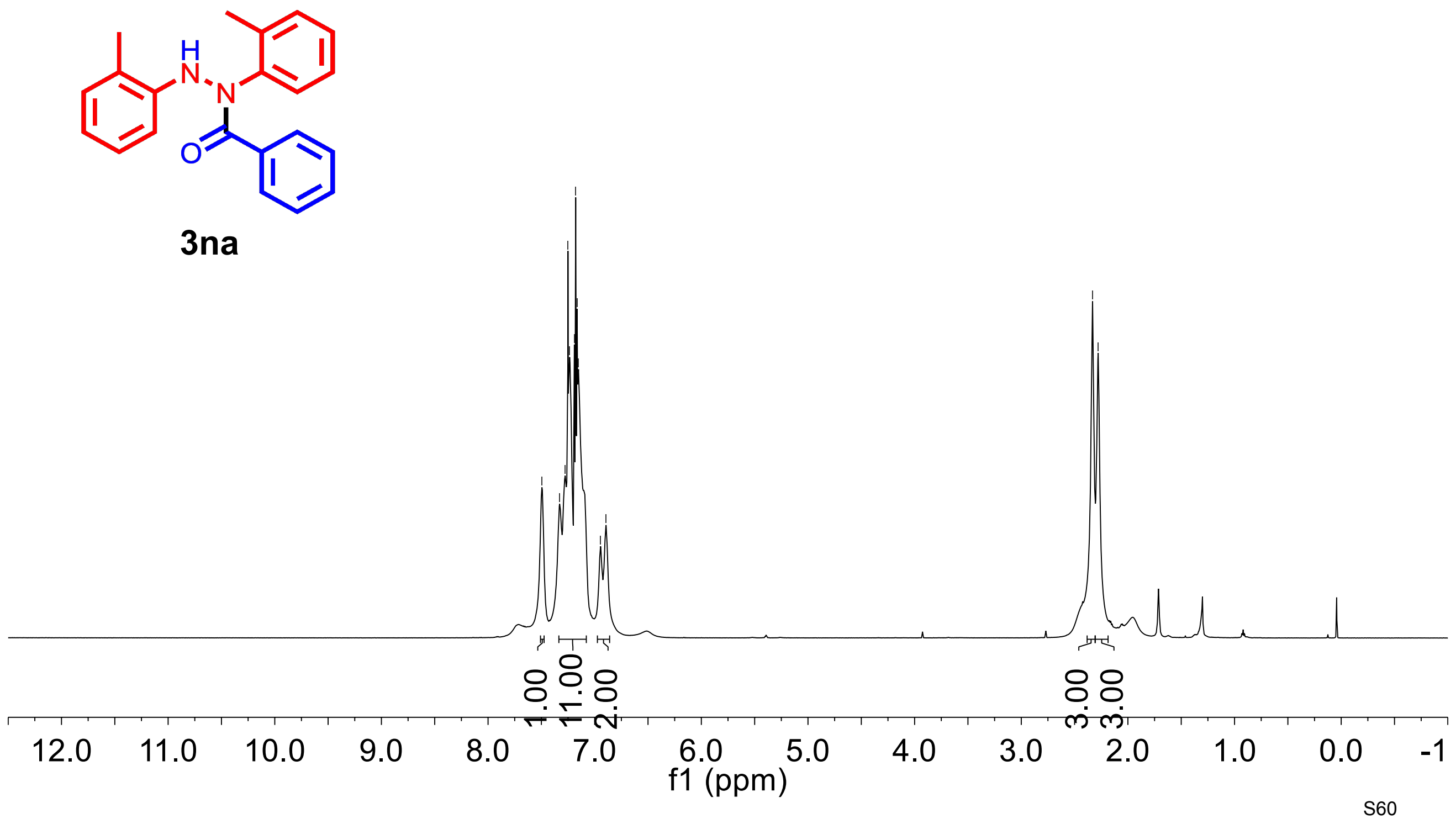




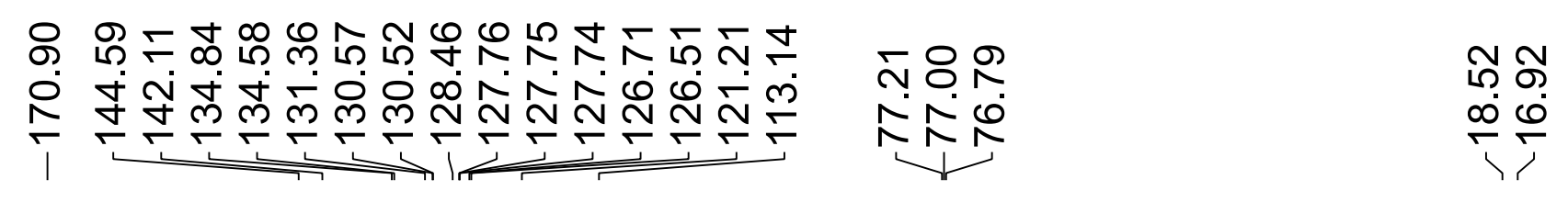

${ }^{13} \mathrm{C}$ NMR $\left(150 \mathrm{MHz}, \mathrm{CDCl}_{3}\right)$

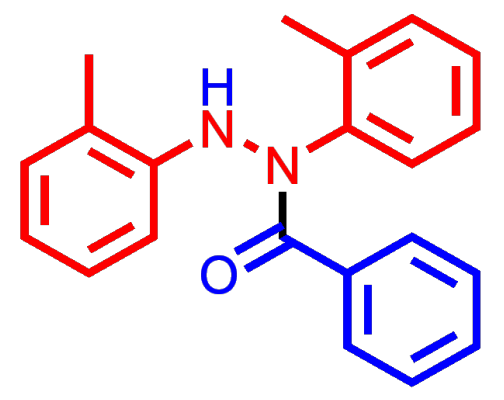

3na

\begin{tabular}{|c|c|c|c|c|c|c|c|c|c|c|c|c|c|c|c|c|}
\hline 230 & 210 & 190 & 170 & 150 & 130 & $\begin{array}{c}110 \\
\mathrm{f} 1(\mathrm{ppm})\end{array}$ & 90 & 80 & 70 & 60 & 50 & 40 & 30 & 20 & 10 & $\begin{array}{l}0 \\
\text { S61 }\end{array}$ \\
\hline
\end{tabular}


${ }^{1} \mathrm{H}$ NMR $\left(400 \mathrm{MHz}, \mathrm{CDCl}_{3}\right)$

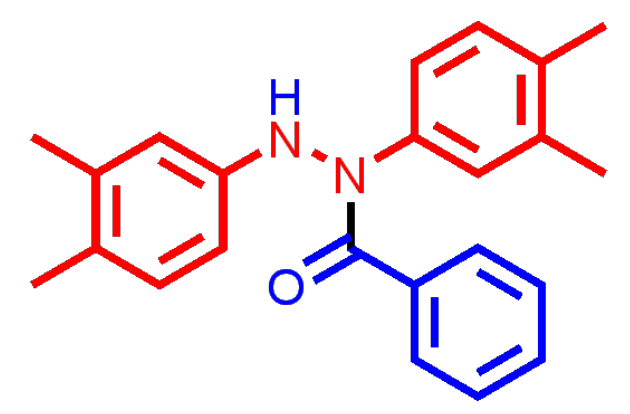

$30 a$

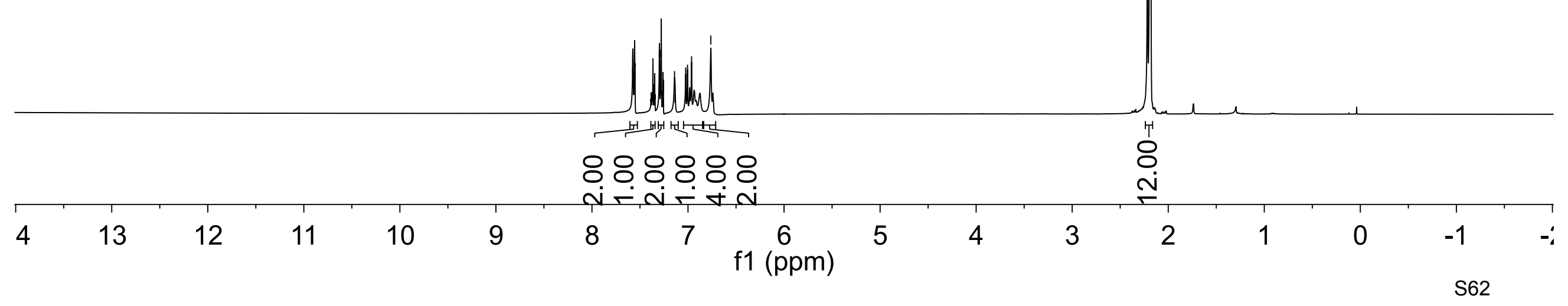


l

Nin

${ }^{13} \mathrm{C}$ NMR $\left(150 \mathrm{MHz}, \mathrm{CDCl}_{3}\right)$

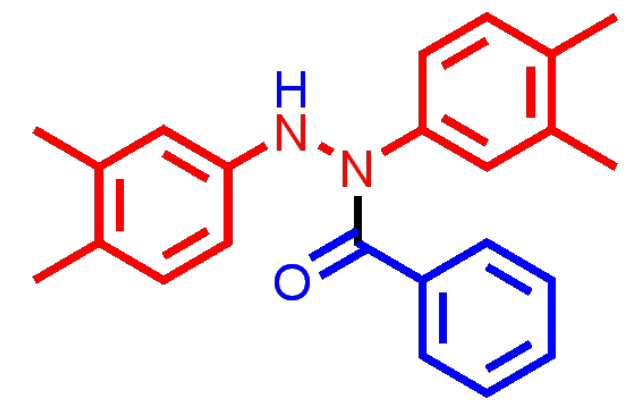

$30 a$ 


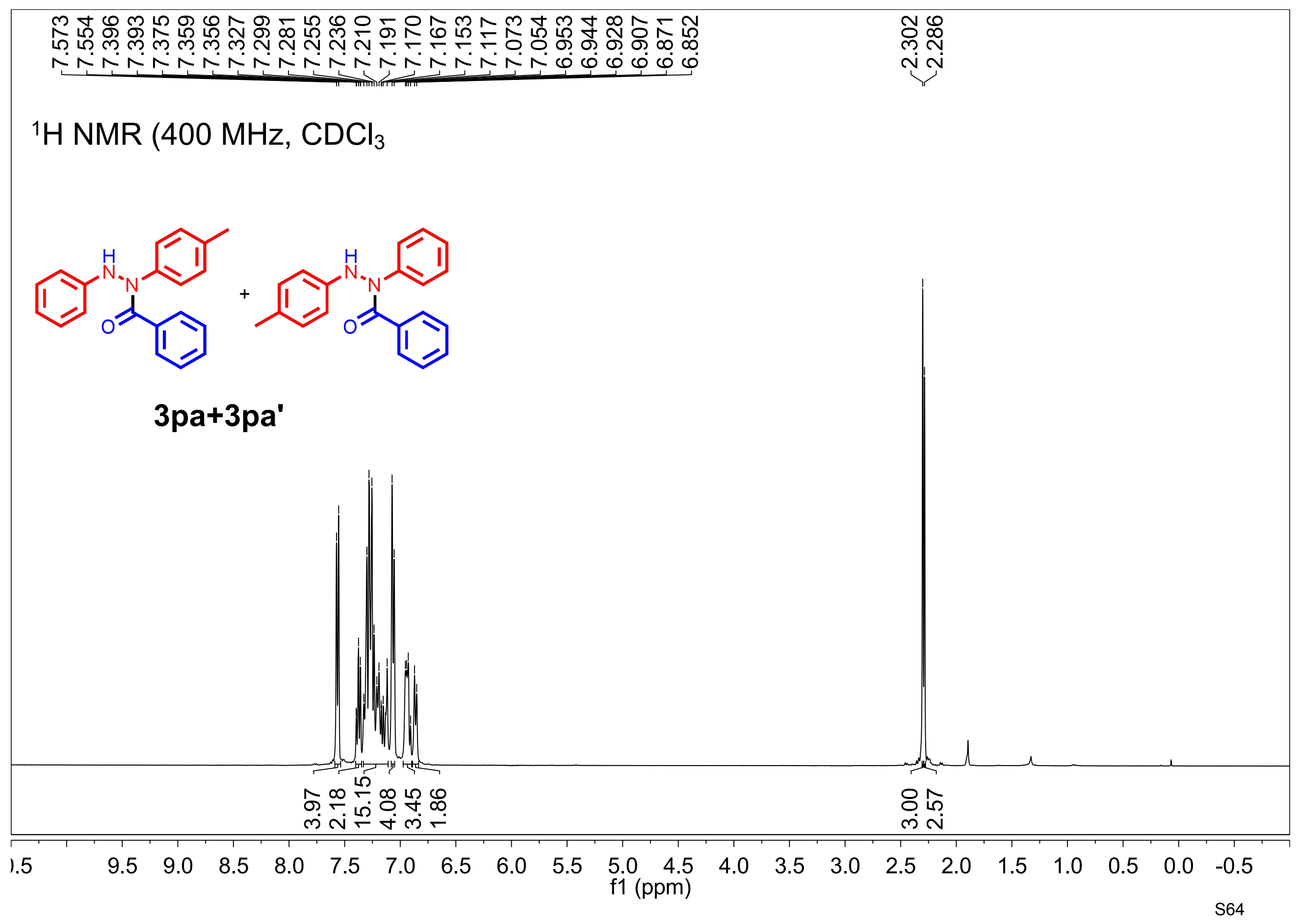




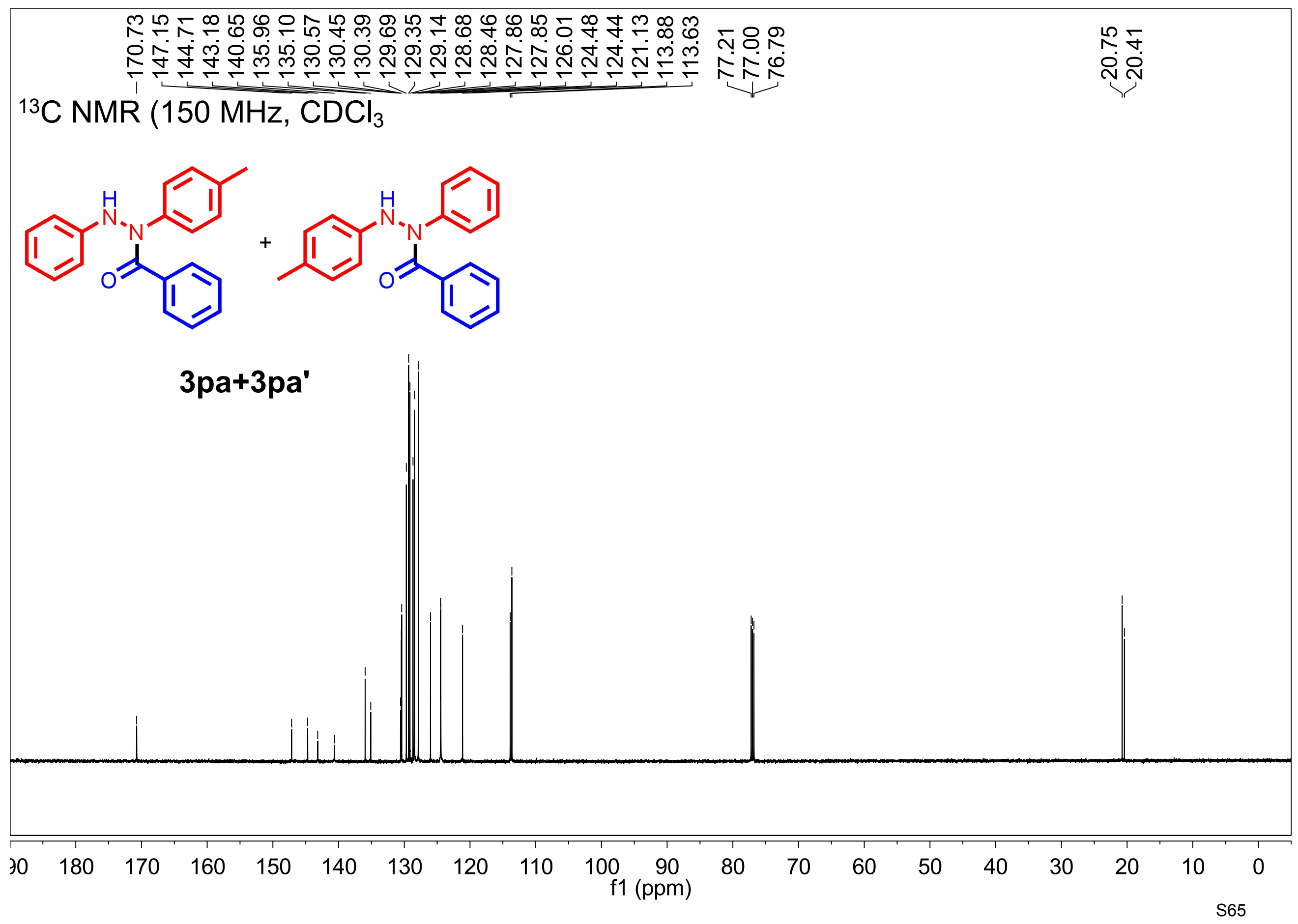




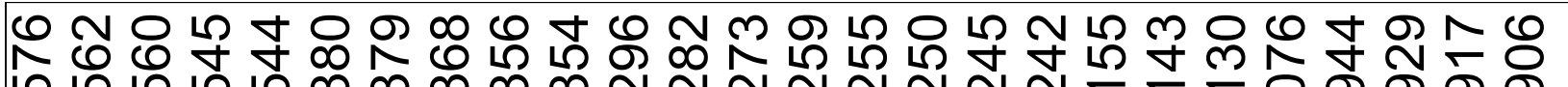
i

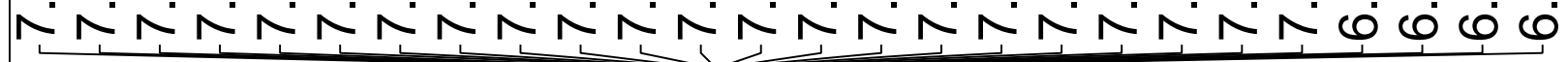

${ }^{1} \mathrm{H}$ NMR $\left(600 \mathrm{MHz}, \mathrm{CDCl}_{3}\right)$

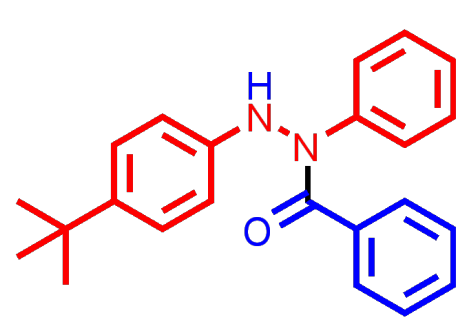

$+$

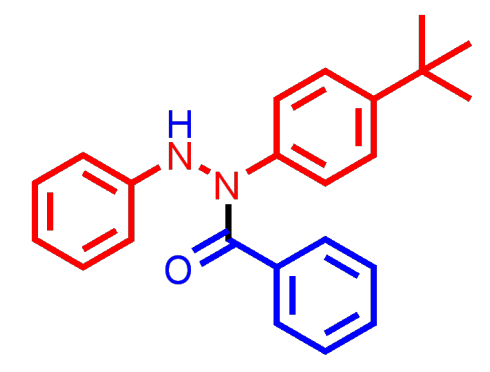

$3 q a+3 q a '$
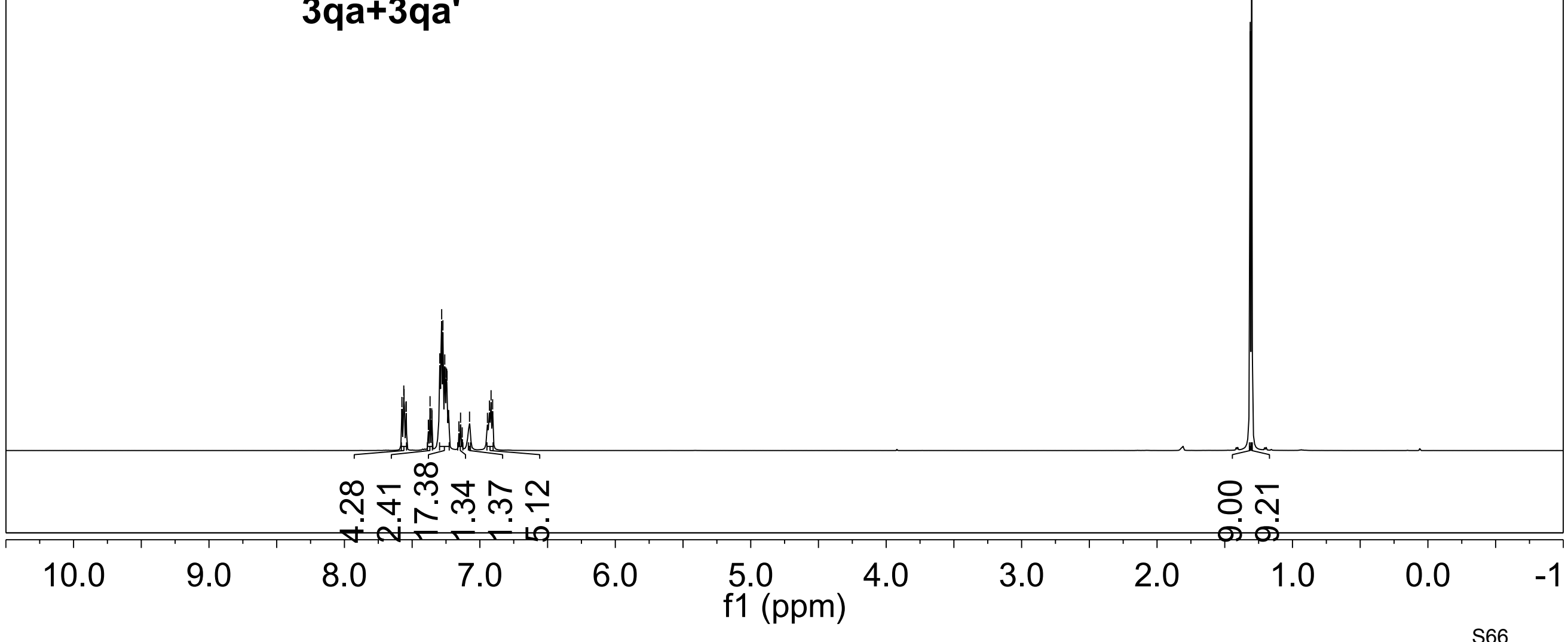


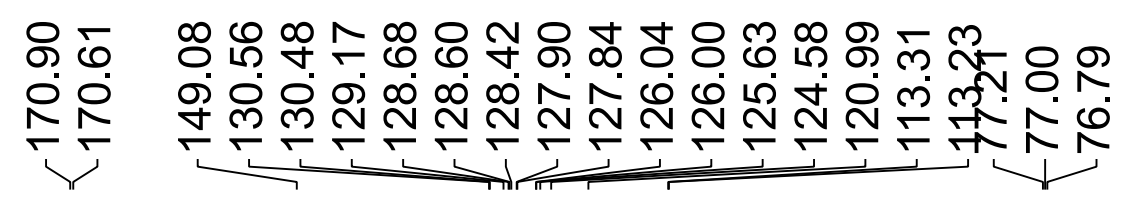

${ }^{13} \mathrm{C}$ NMR (150 MHz, CDCl 3 )

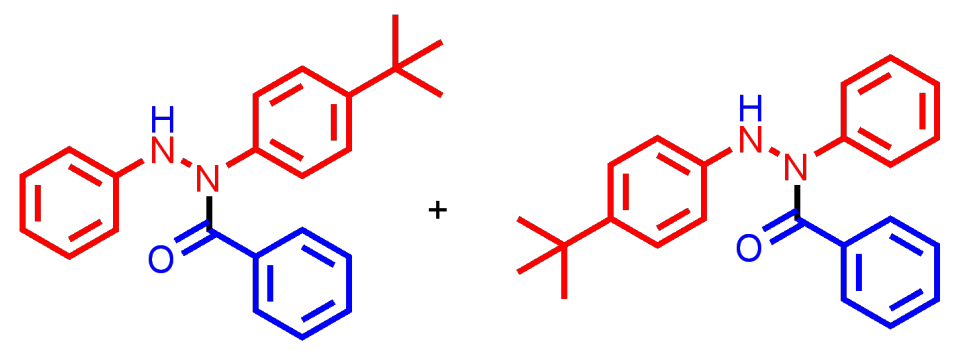

$3 q a+3 q a^{\prime}$

\begin{tabular}{lllllllllllllllll}
\hline 230 & 210 & 190 & 170 & 150 & 130 & $\begin{array}{c}110 \\
\mathrm{f} 1(\mathrm{ppm})\end{array}$ & 90 & 80 & 70 & 60 & 50 & 40 & 30 & 20 & 10 & 0
\end{tabular}


${ }^{1} \mathrm{H}$ NMR $\left(400 \mathrm{MHz}, \mathrm{CDCl}_{3}\right)$

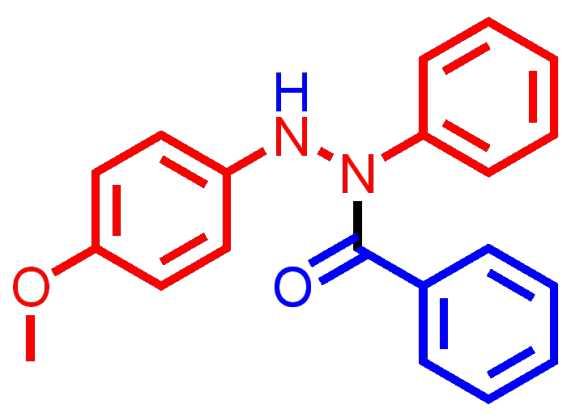

3ra

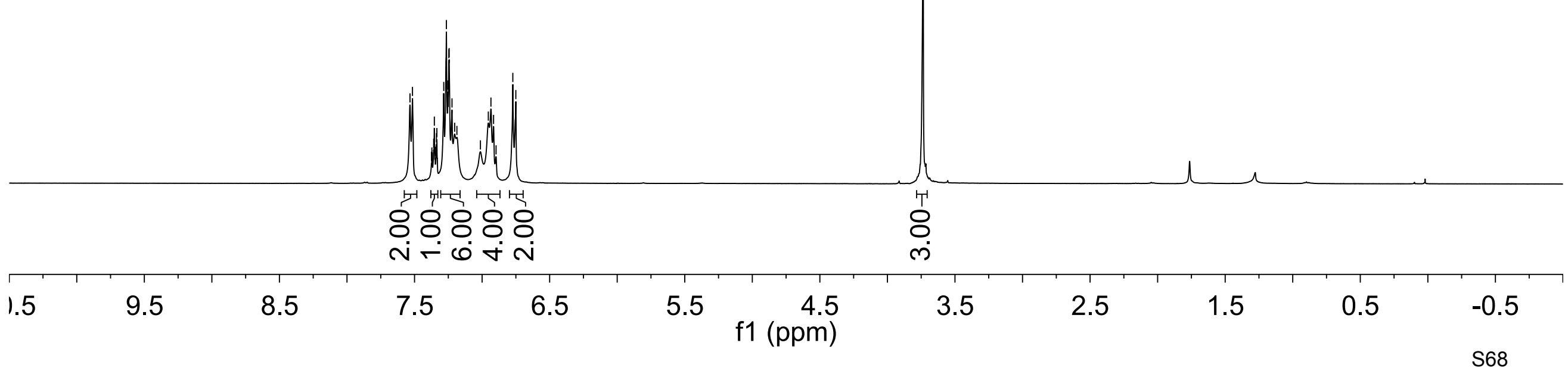




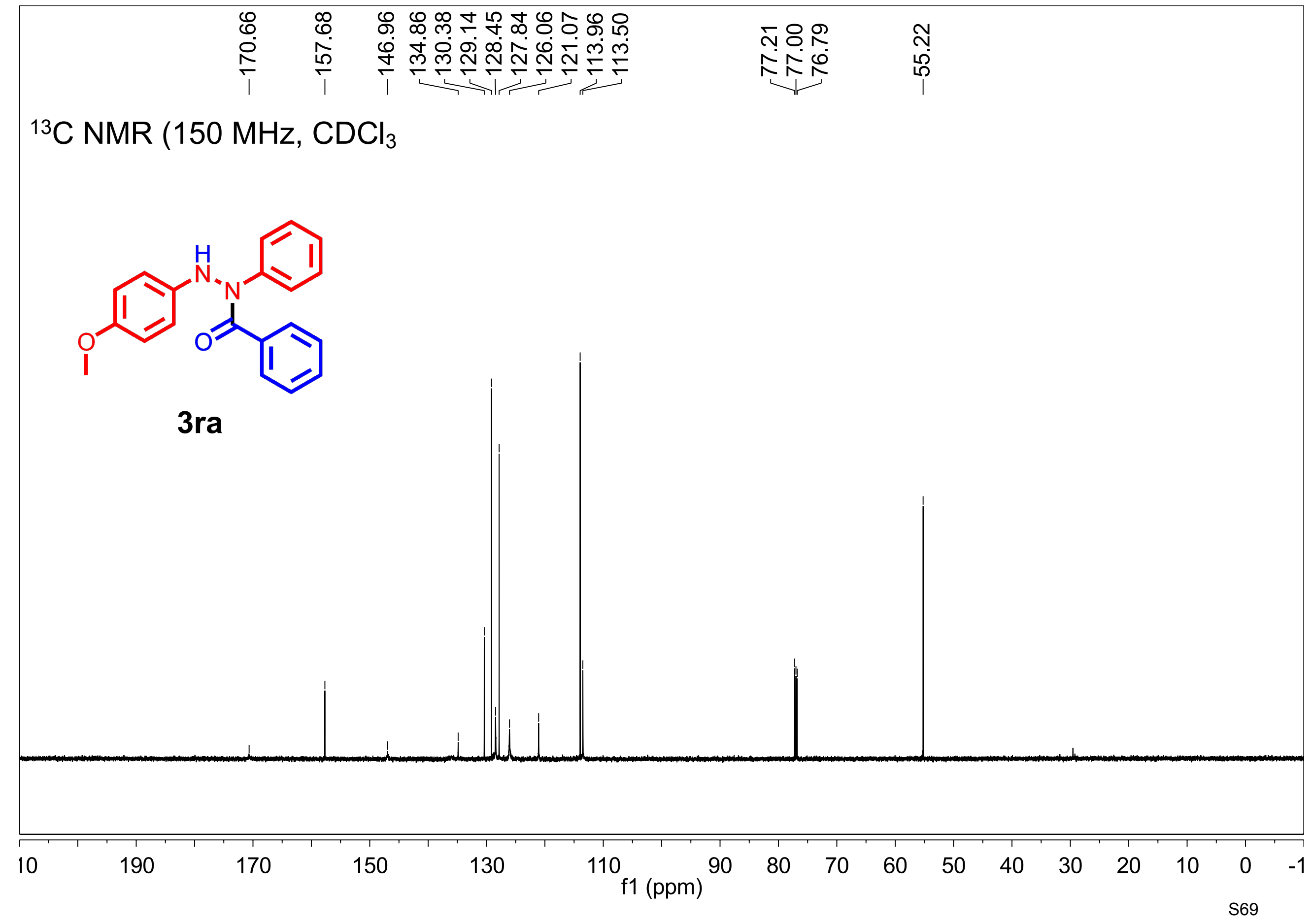




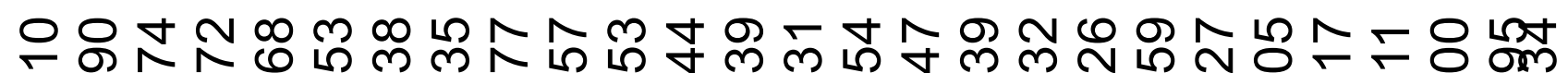
हึ

N

${ }^{1} \mathrm{H}$ NMR (400 MHz, $\mathrm{CDCl}_{3}$ )

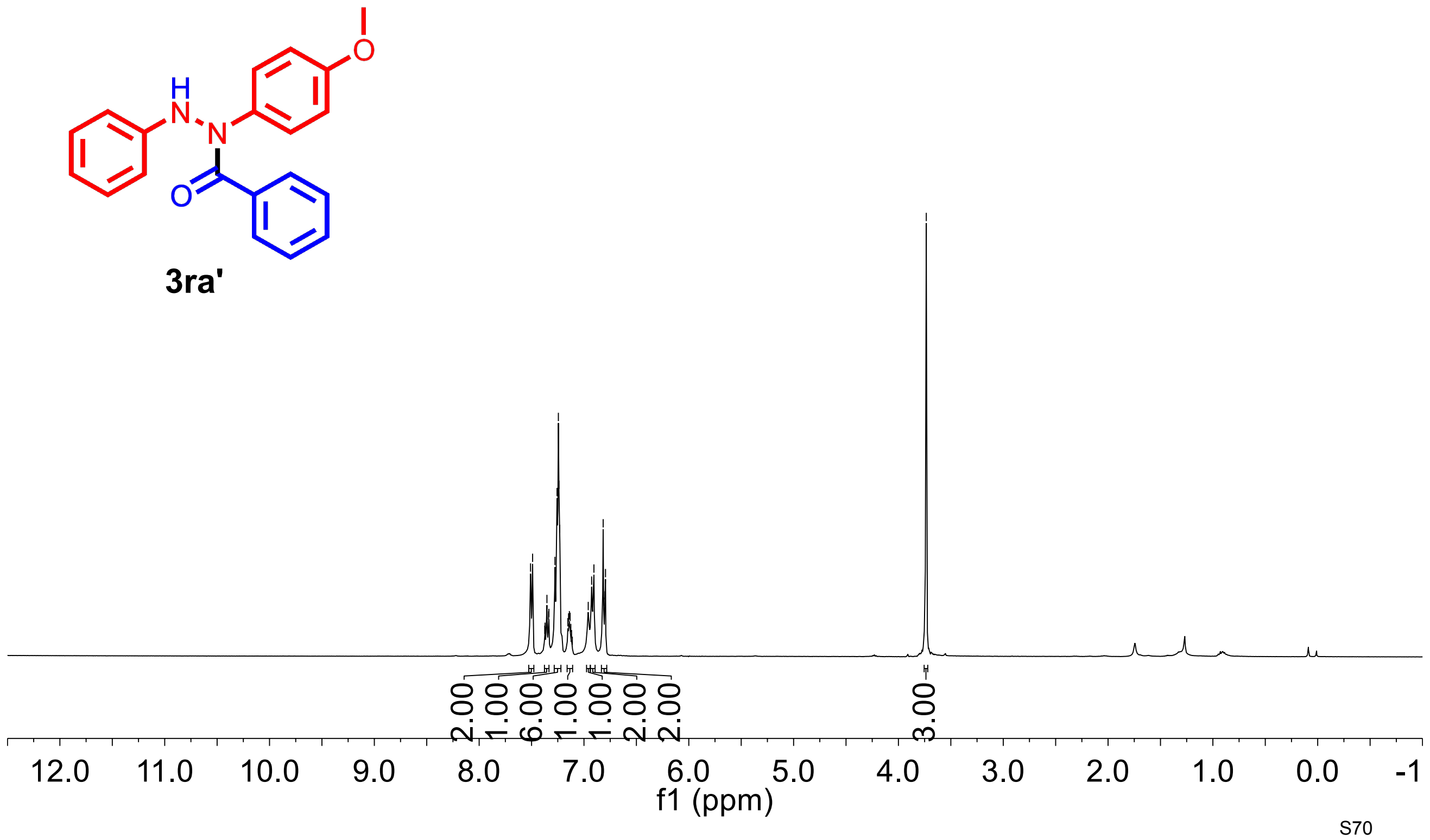




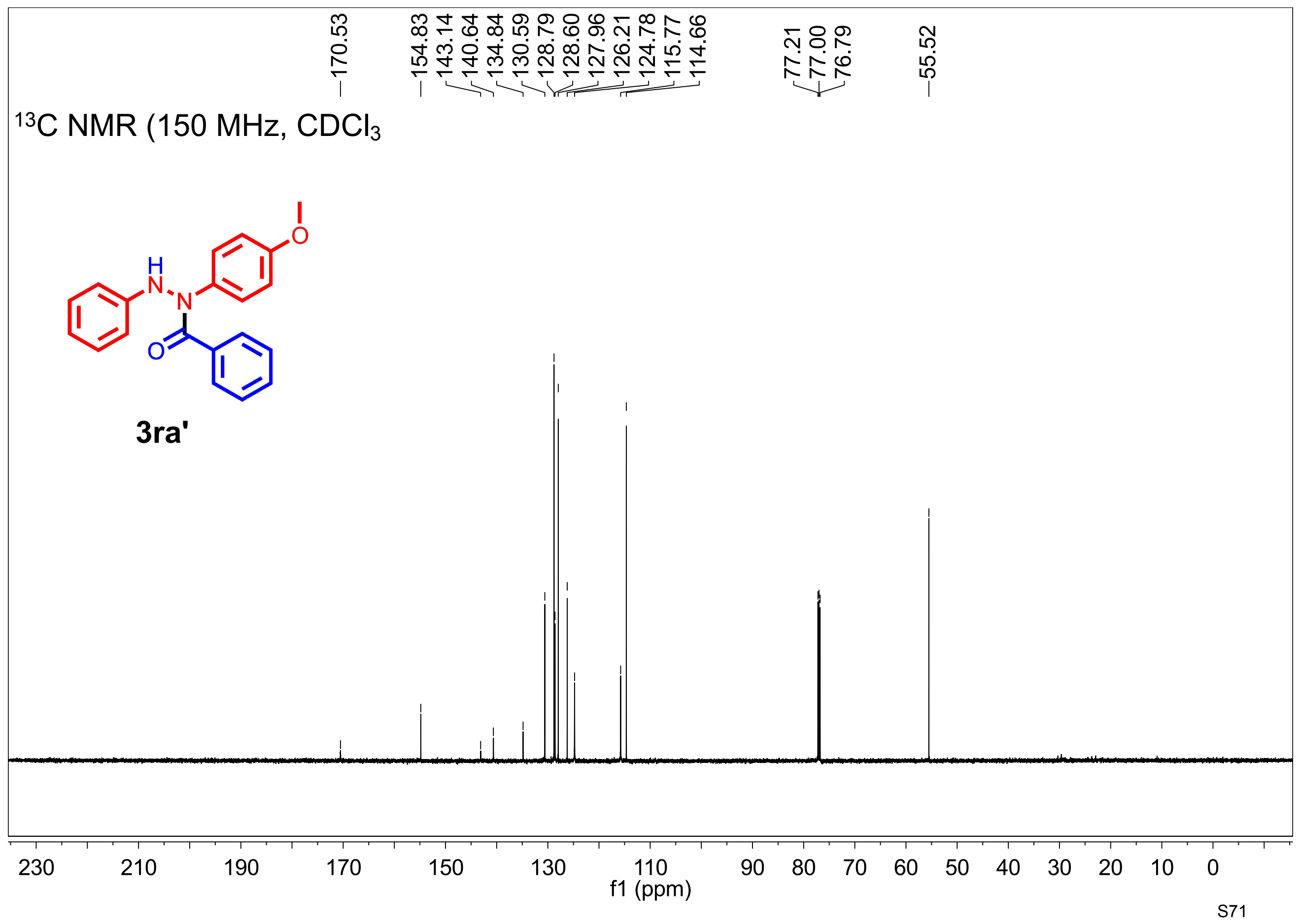


${ }^{1} \mathrm{H}$ NMR $\left(400 \mathrm{MHz}, \mathrm{CDCl}_{3}\right)$

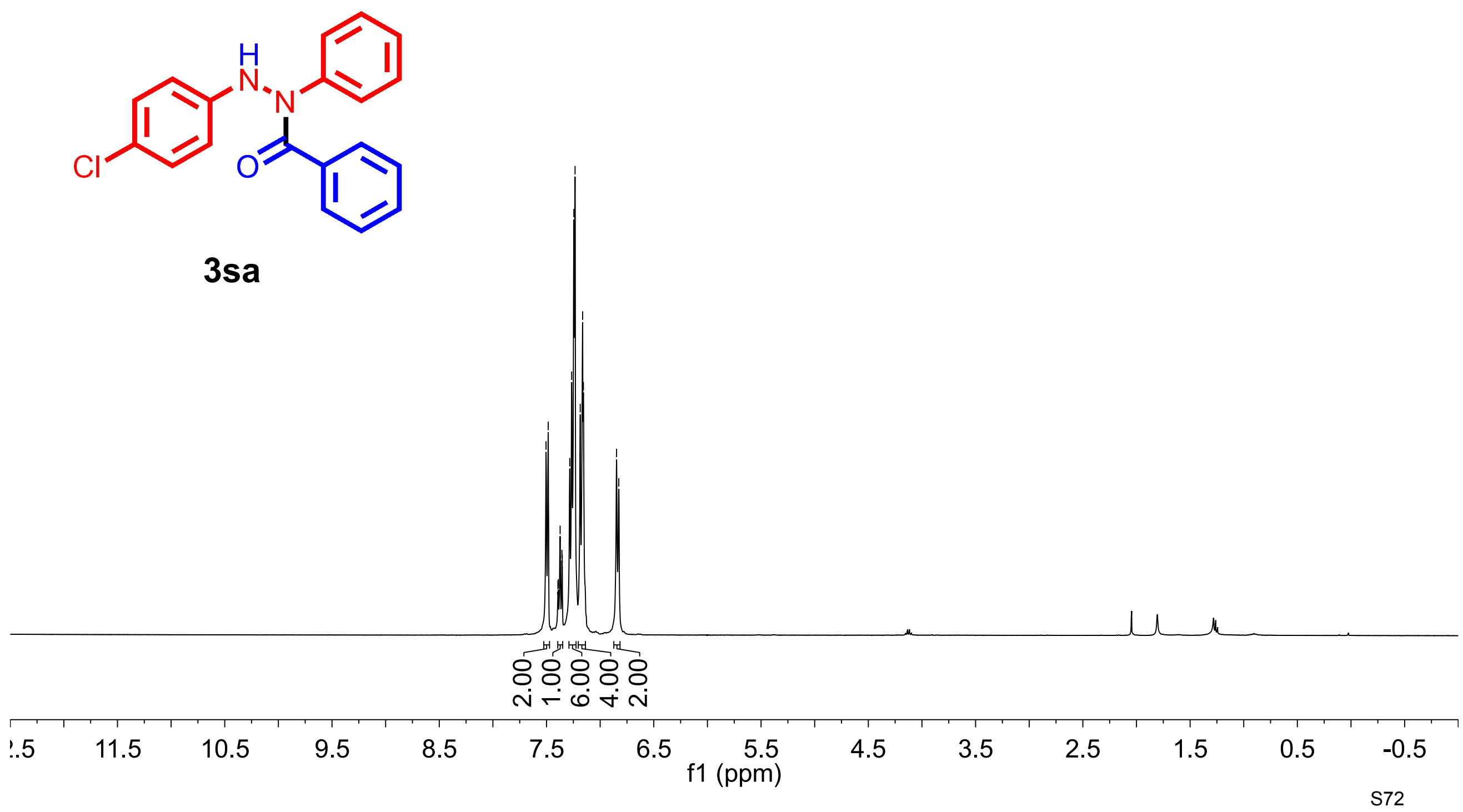




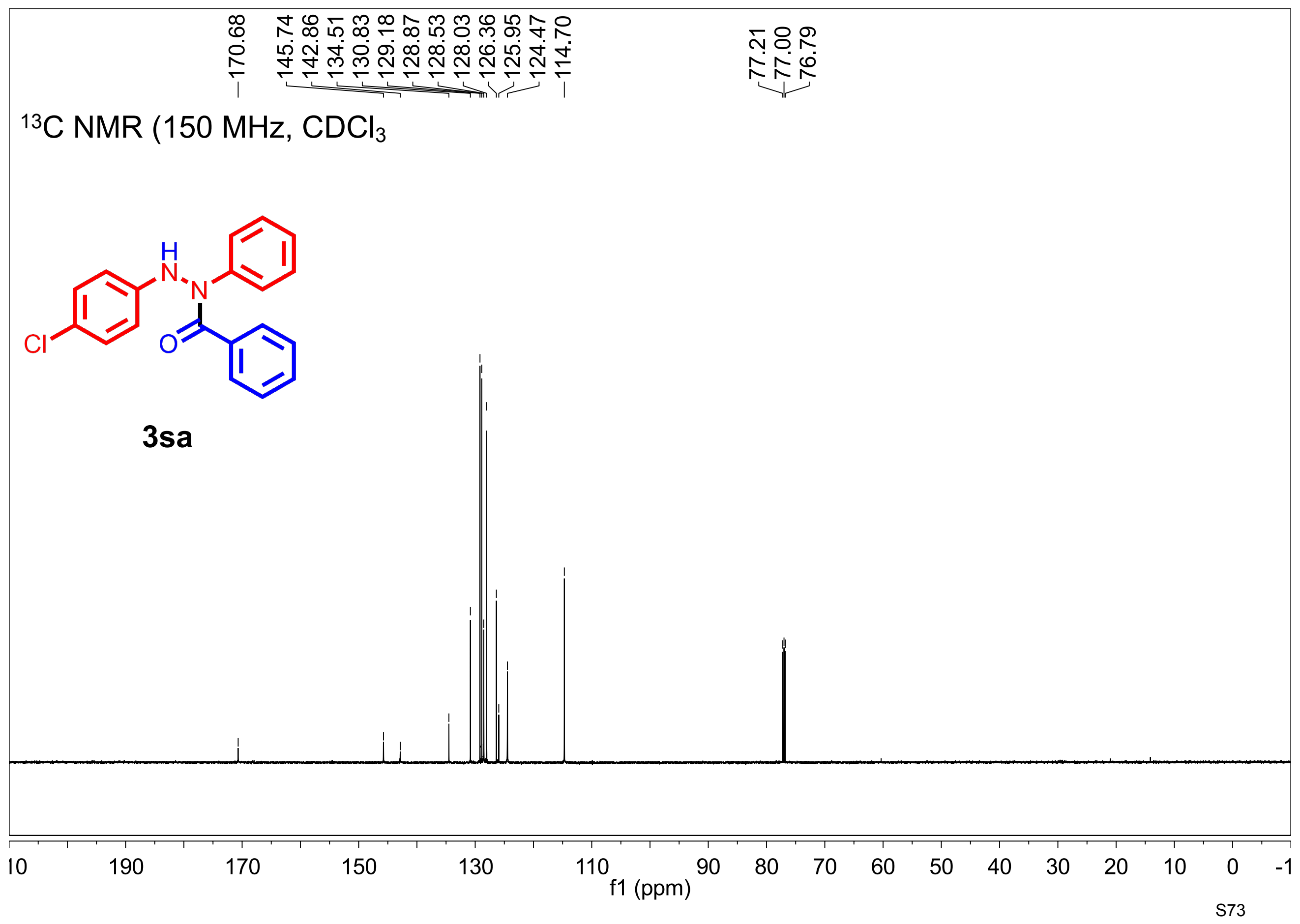




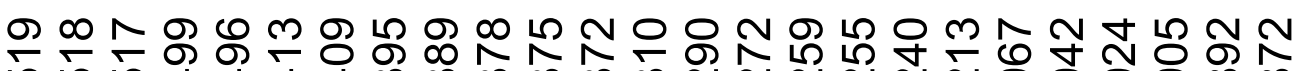

ह

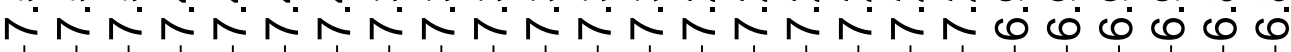

${ }^{1} \mathrm{H}$ NMR $\left(400 \mathrm{MHz}, \mathrm{CDCl}_{3}\right)$

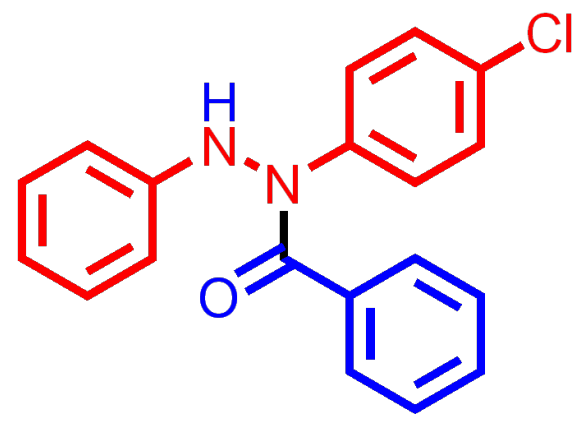

3sa'

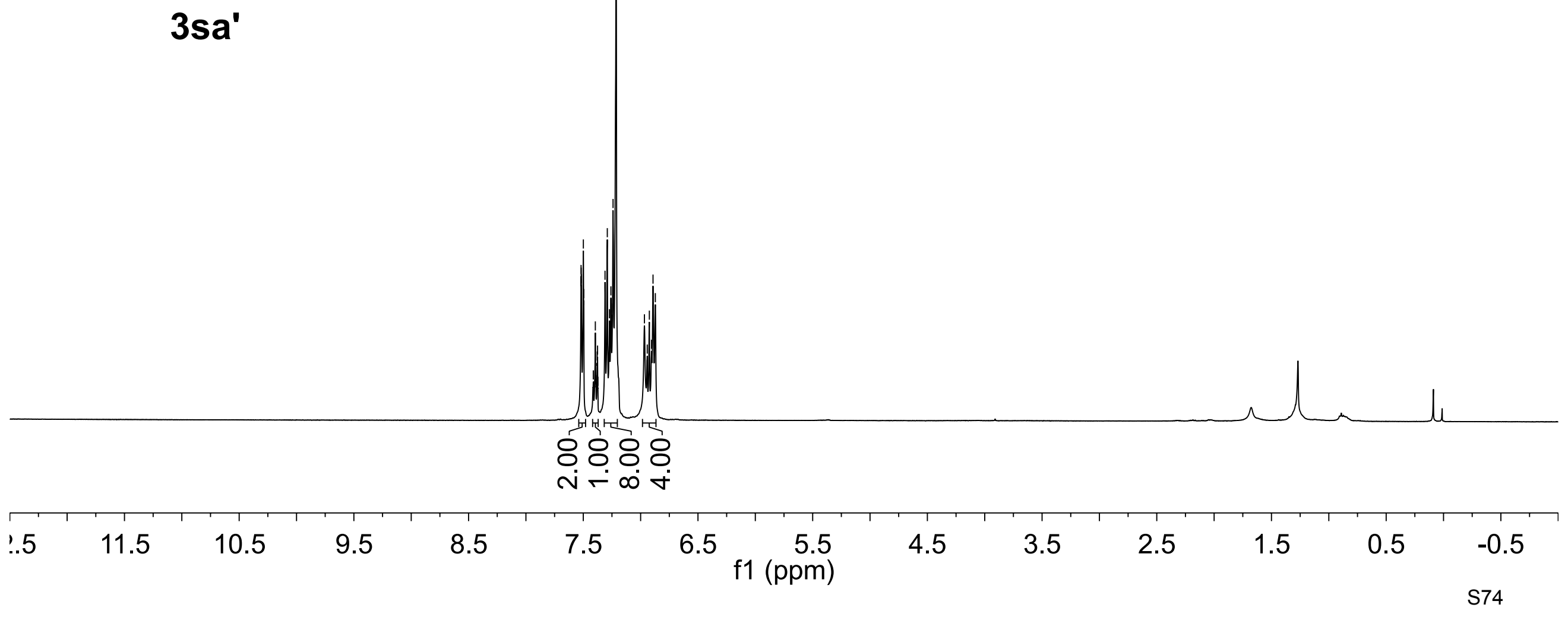




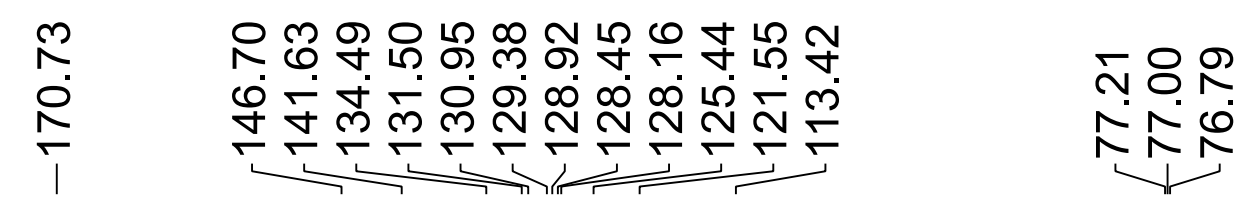

${ }^{13} \mathrm{C}$ NMR (150 MHz, $\mathrm{CDCl}_{3}$ )

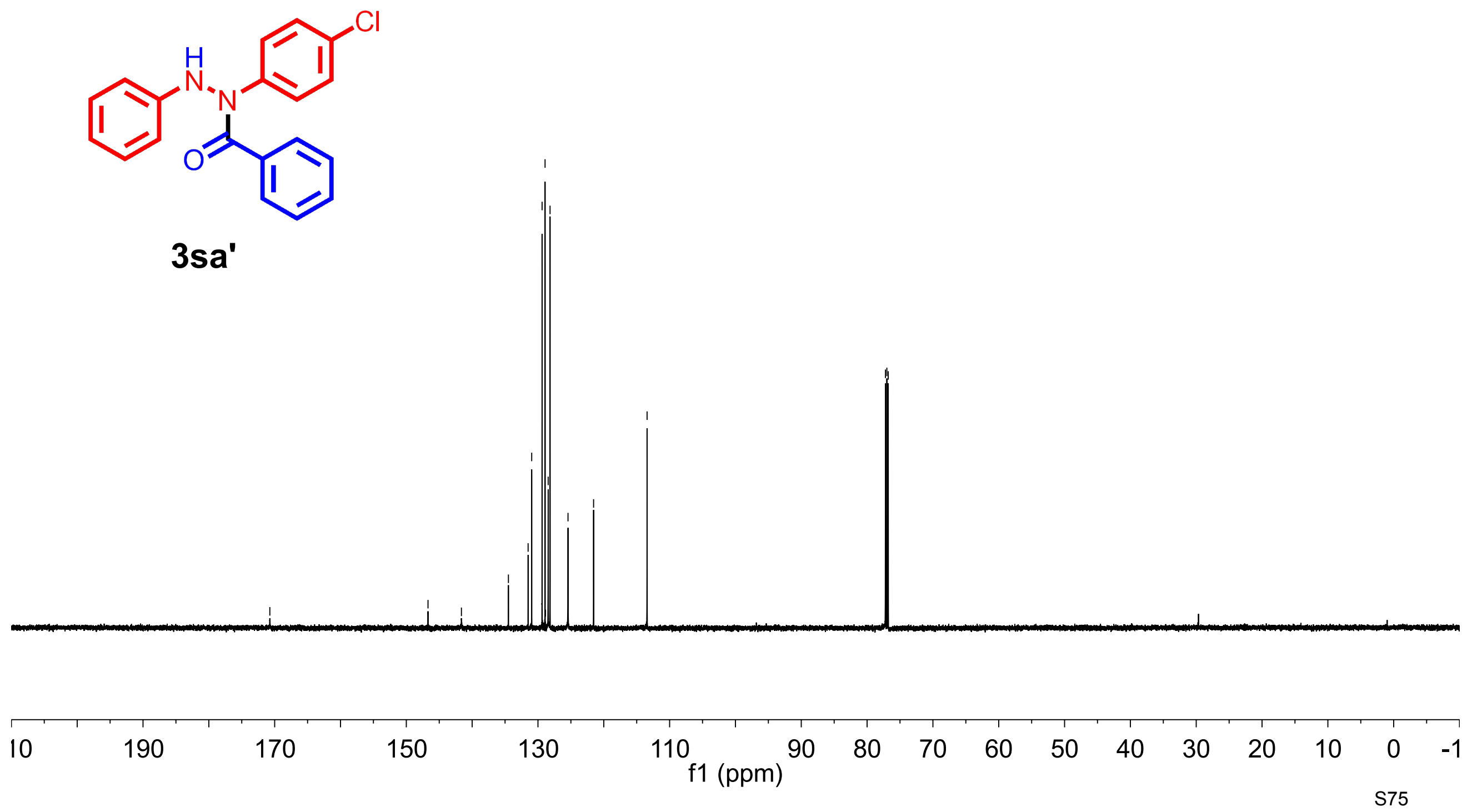




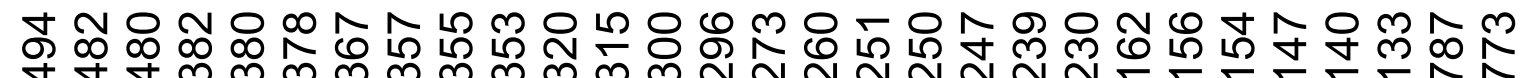

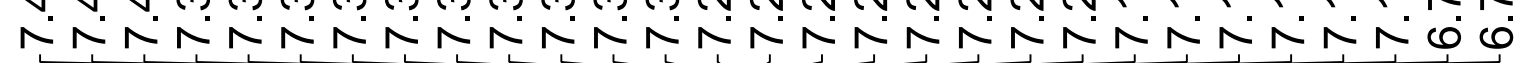

${ }^{1} \mathrm{H}$ NMR $\left(600 \mathrm{MHz}, \mathrm{CDCl}_{3}\right)$

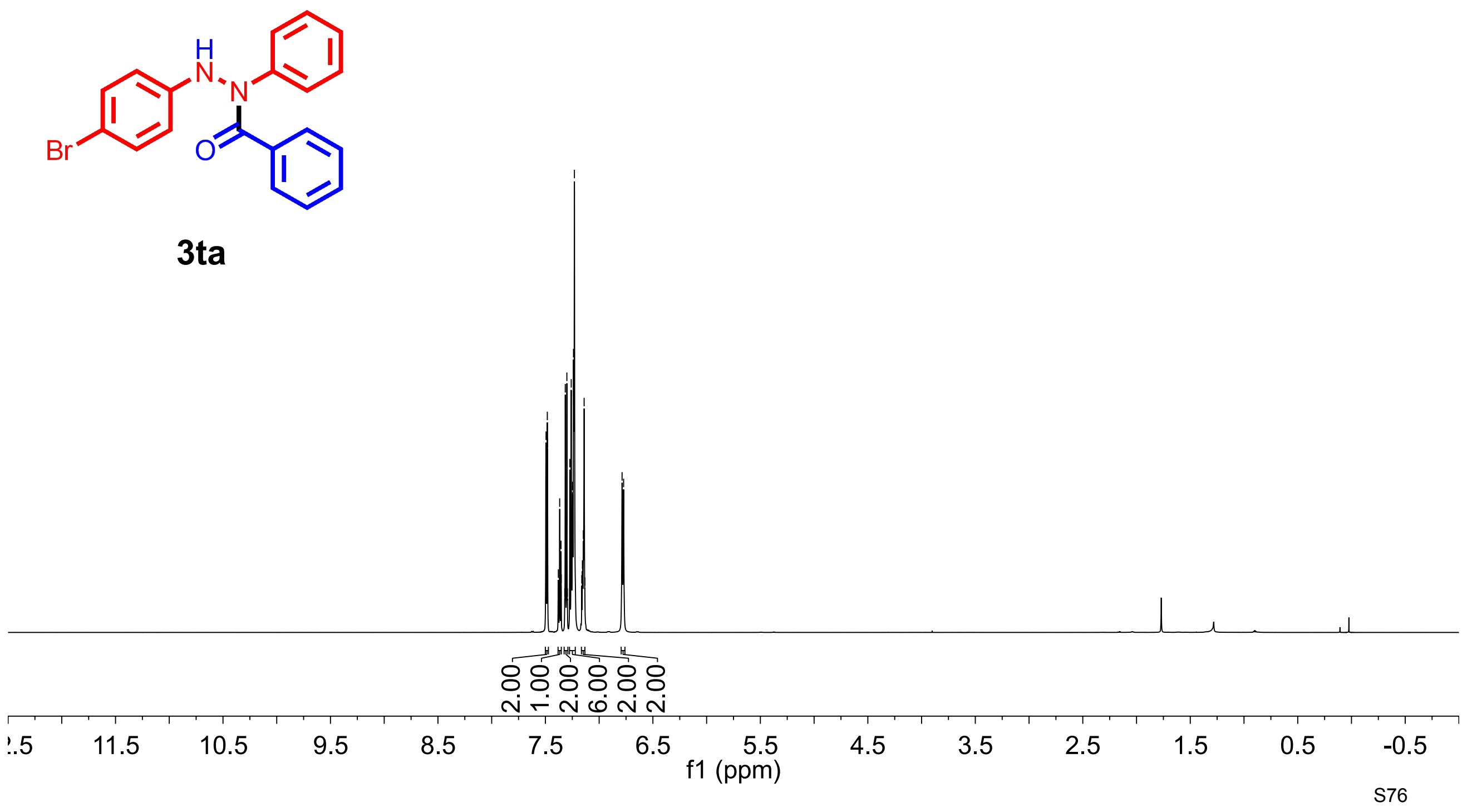


œ స

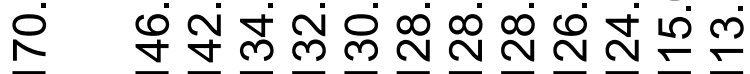

i

సัㅇำ

กิ์

N

${ }^{13} \mathrm{C}$ NMR (150 MHz, $\left.\mathrm{CDCl}_{3}\right)$

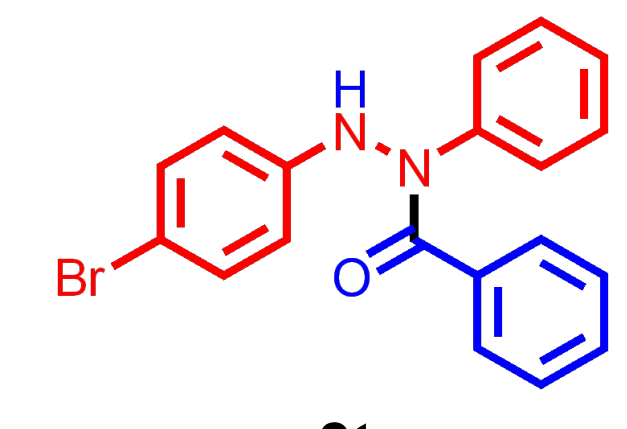

3 ta

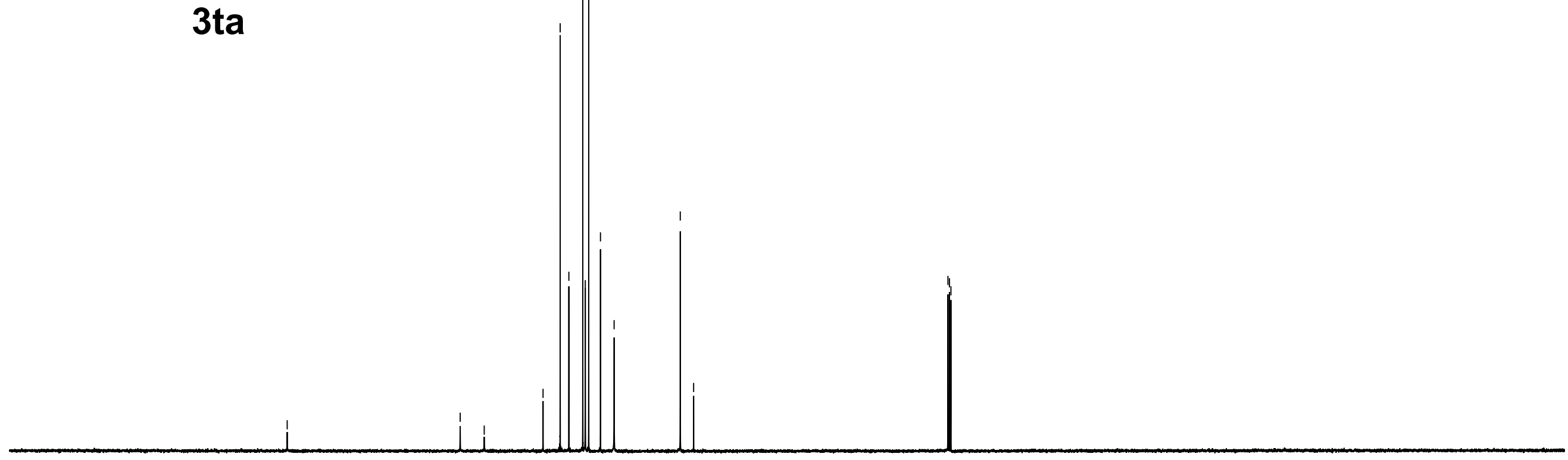

10

200

190

180 


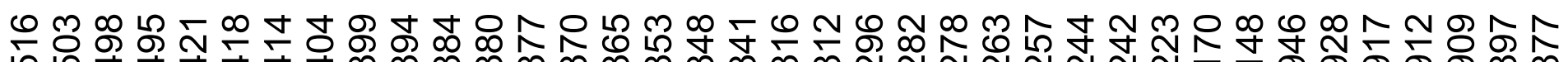

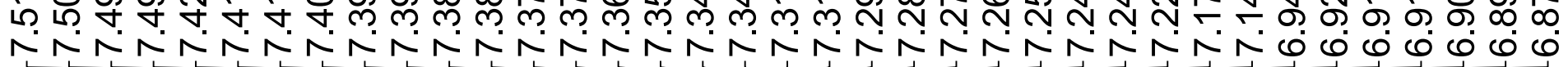

\section{${ }^{1} \mathrm{H}$ NMR $\left(400 \mathrm{MHz}, \mathrm{CDCl}_{3}\right)$}<smiles>O=C(c1ccccc1)N(Nc1ccccc1)c1ccc(Br)cc1</smiles>

3ta'

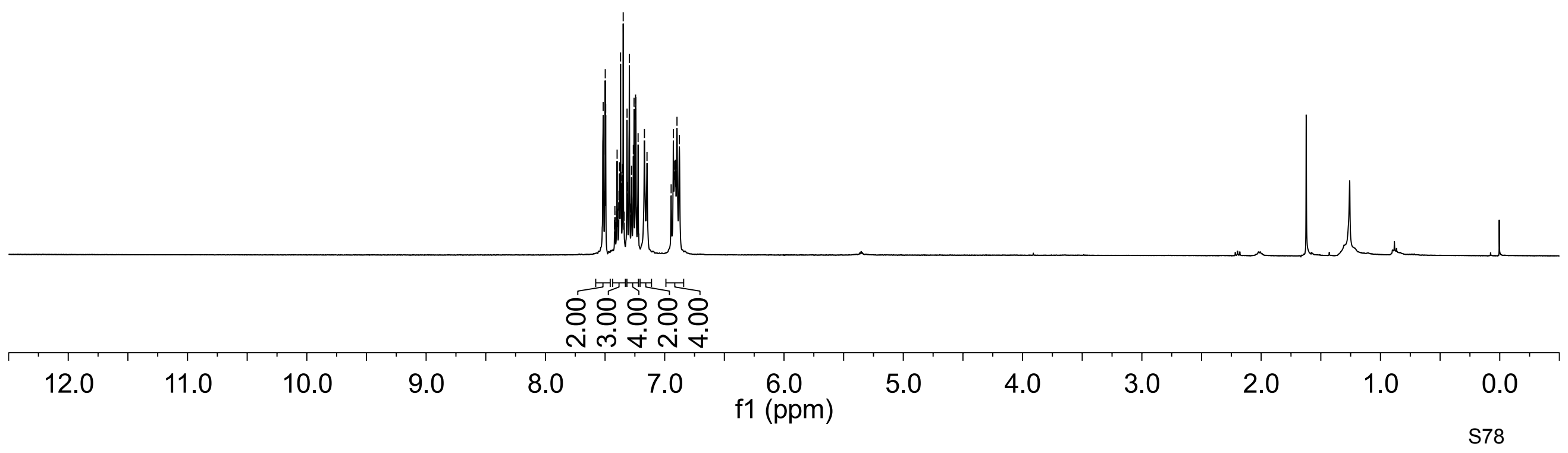




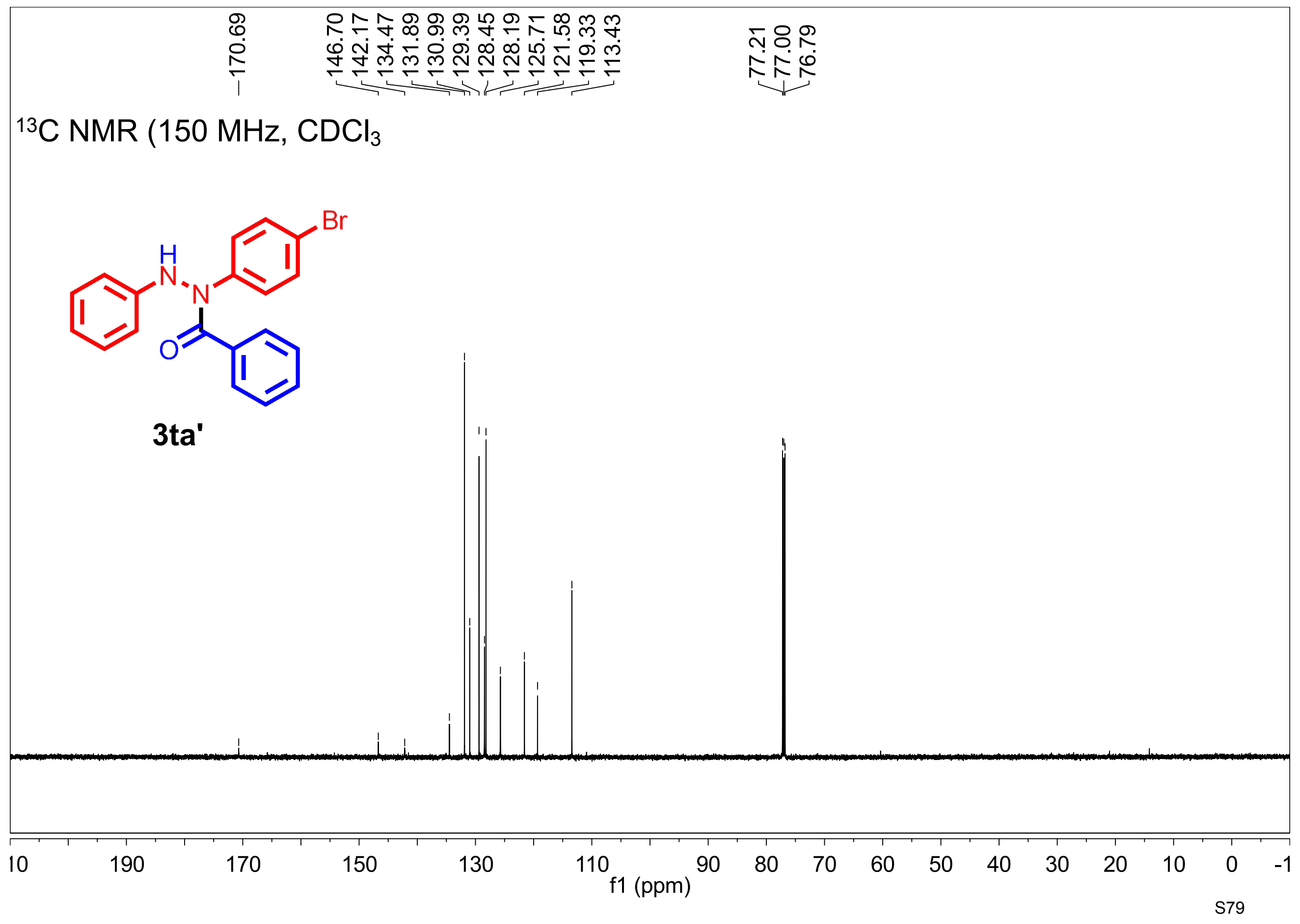


${ }^{1} \mathrm{H}$ NMR $\left(600 \mathrm{MHz}, \mathrm{CDCl}_{3}\right)$

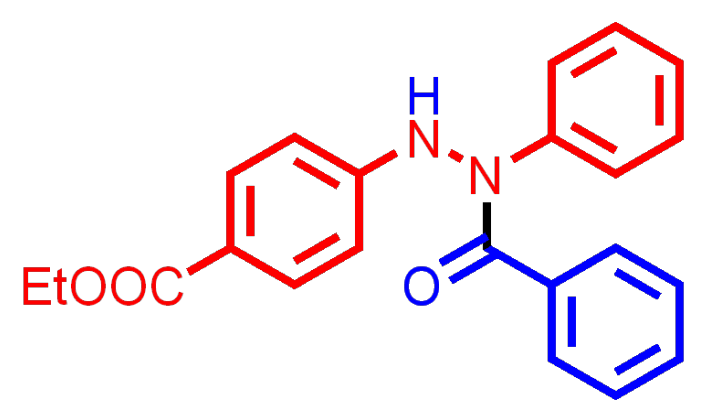

3ua

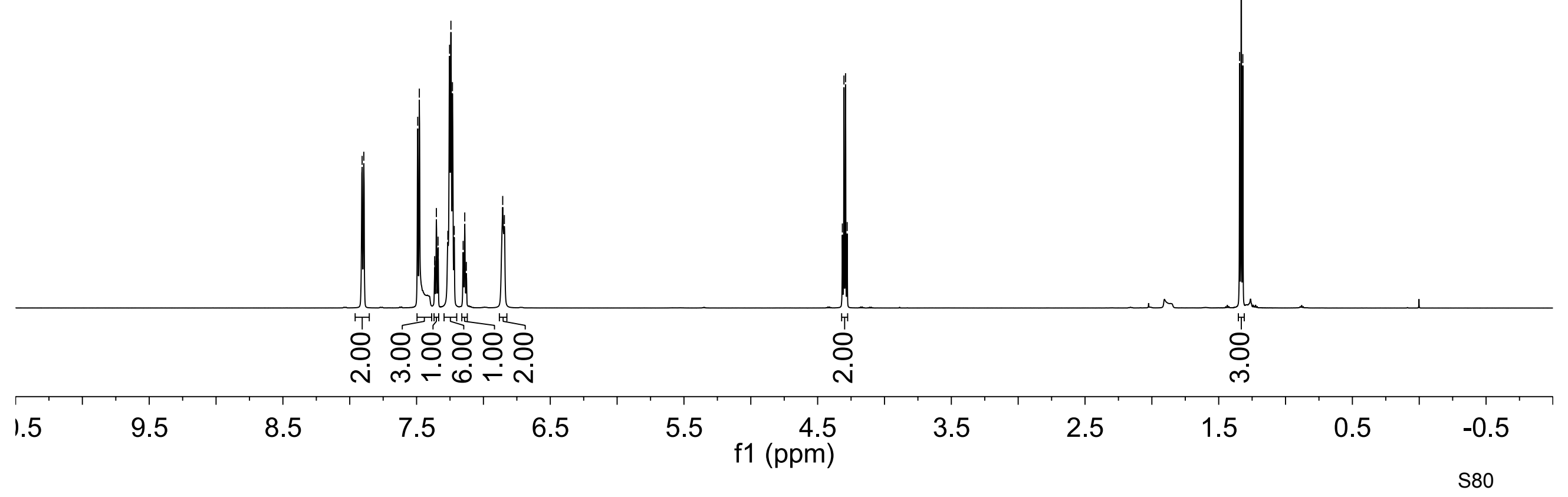




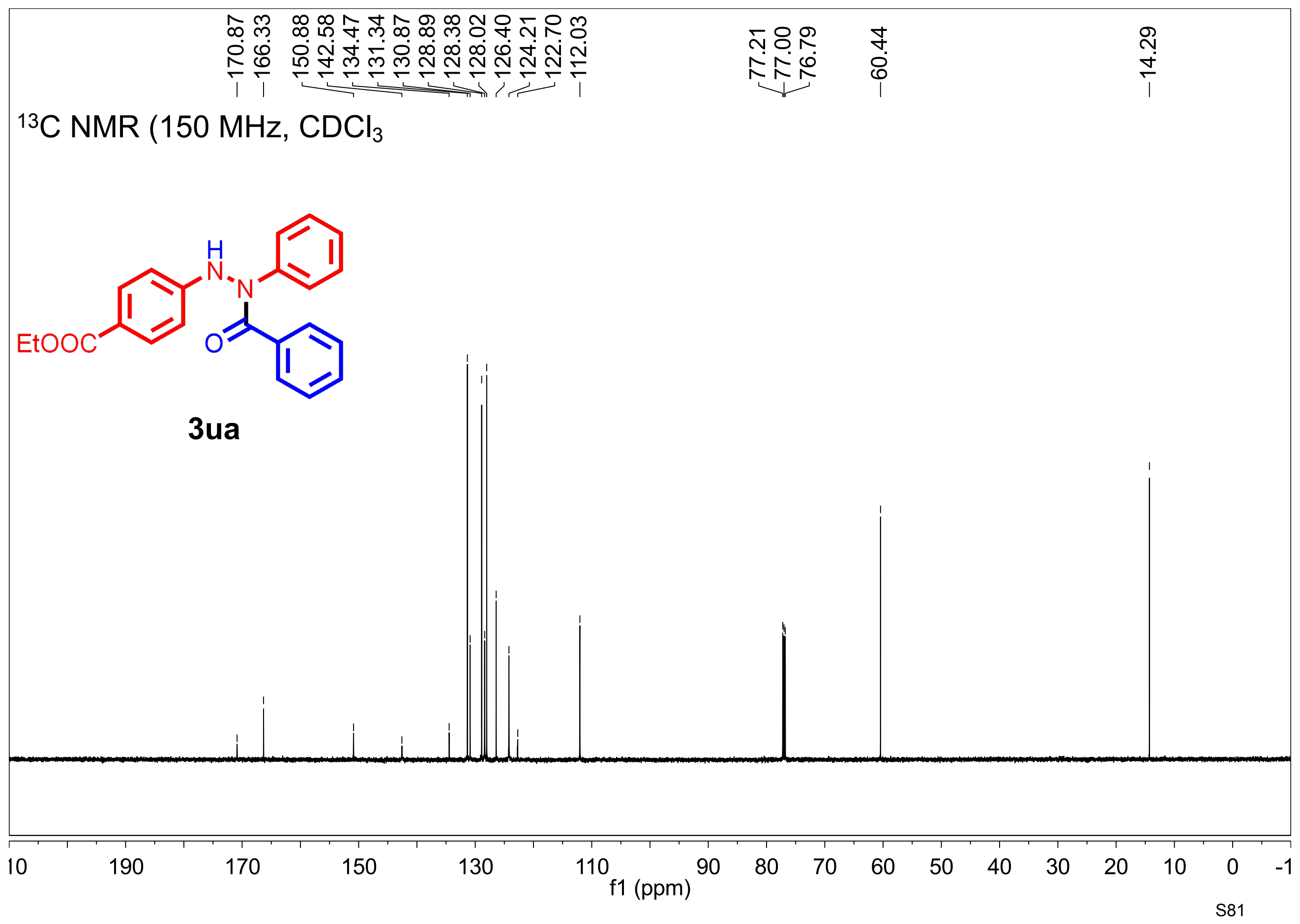




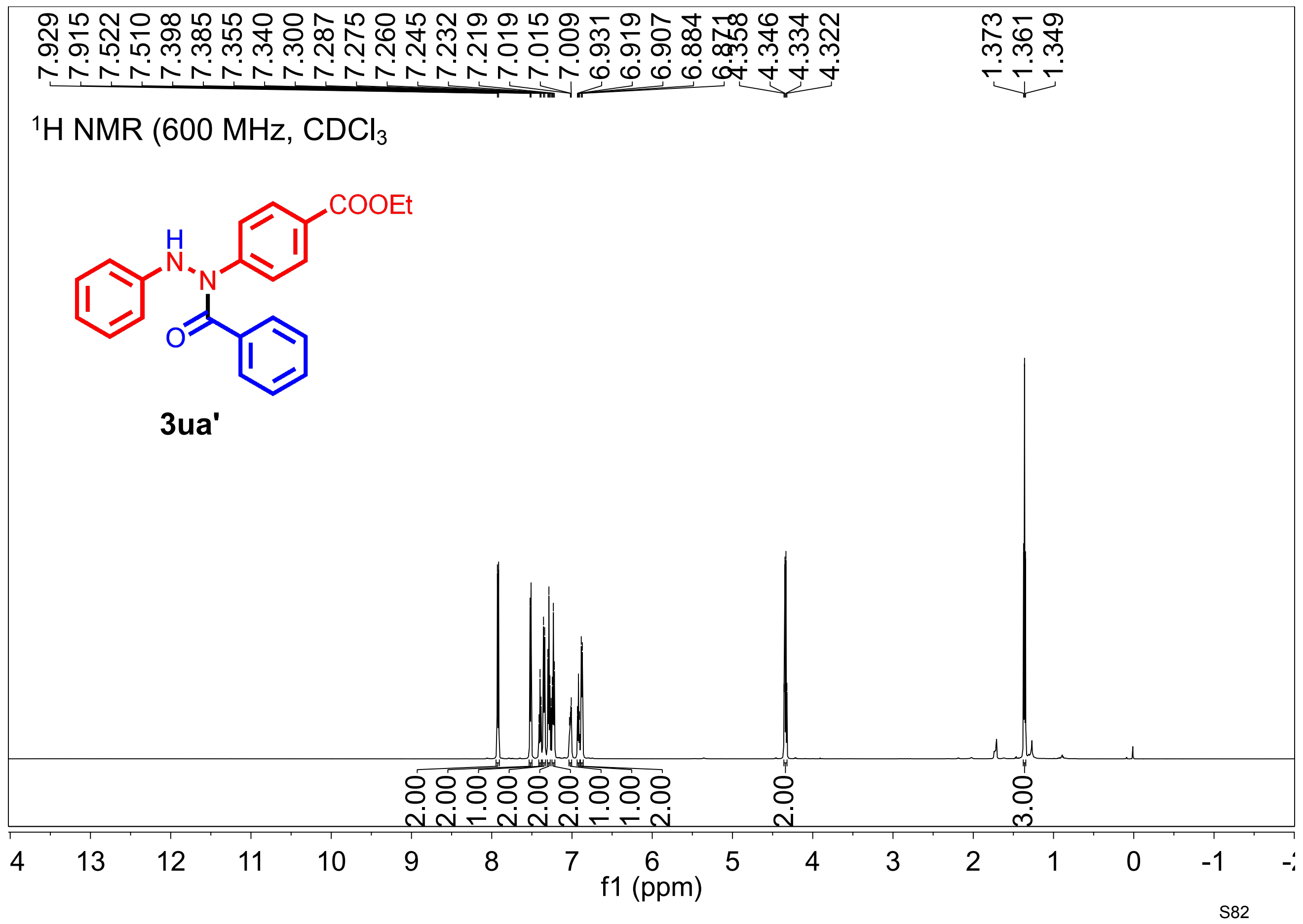




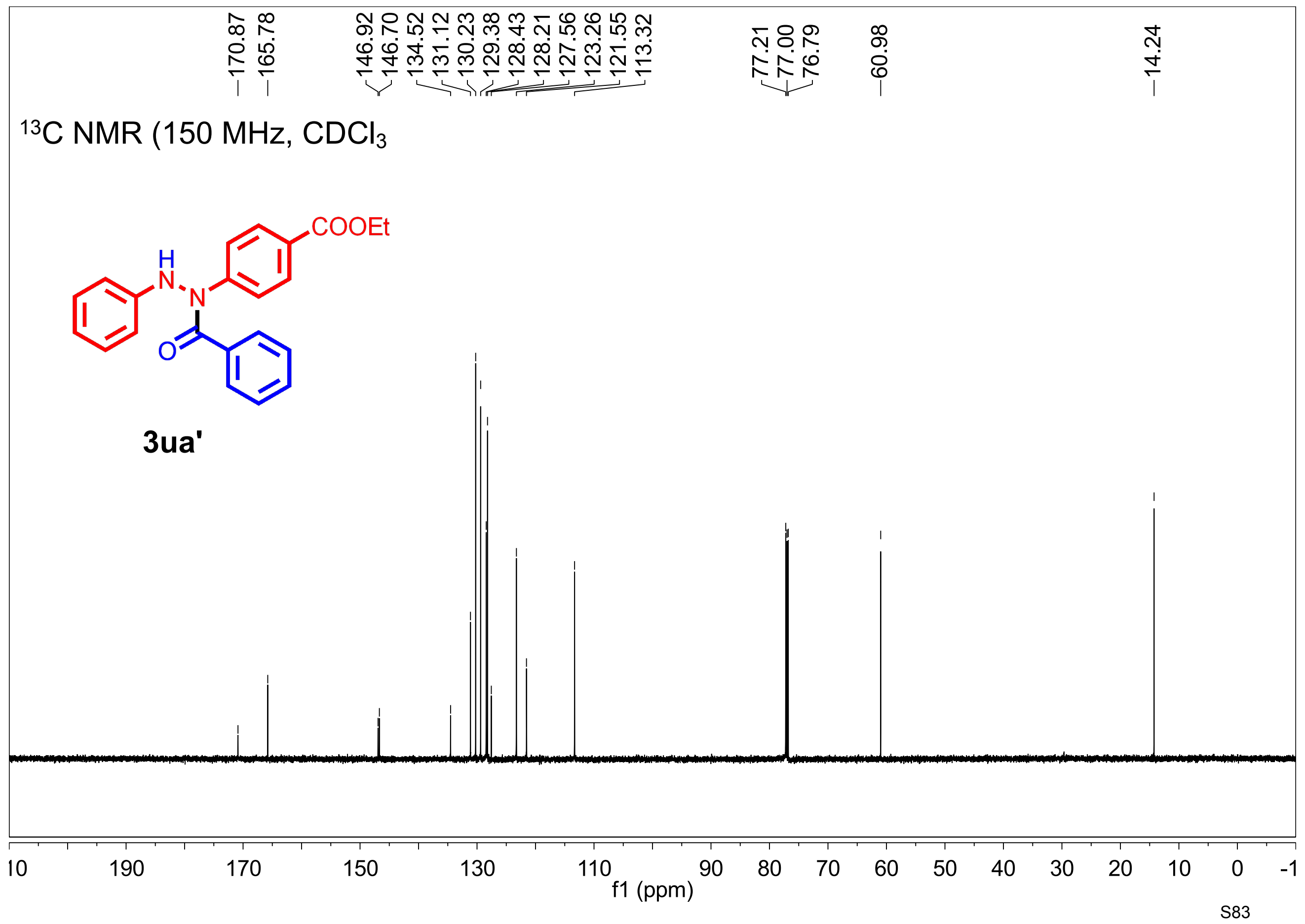




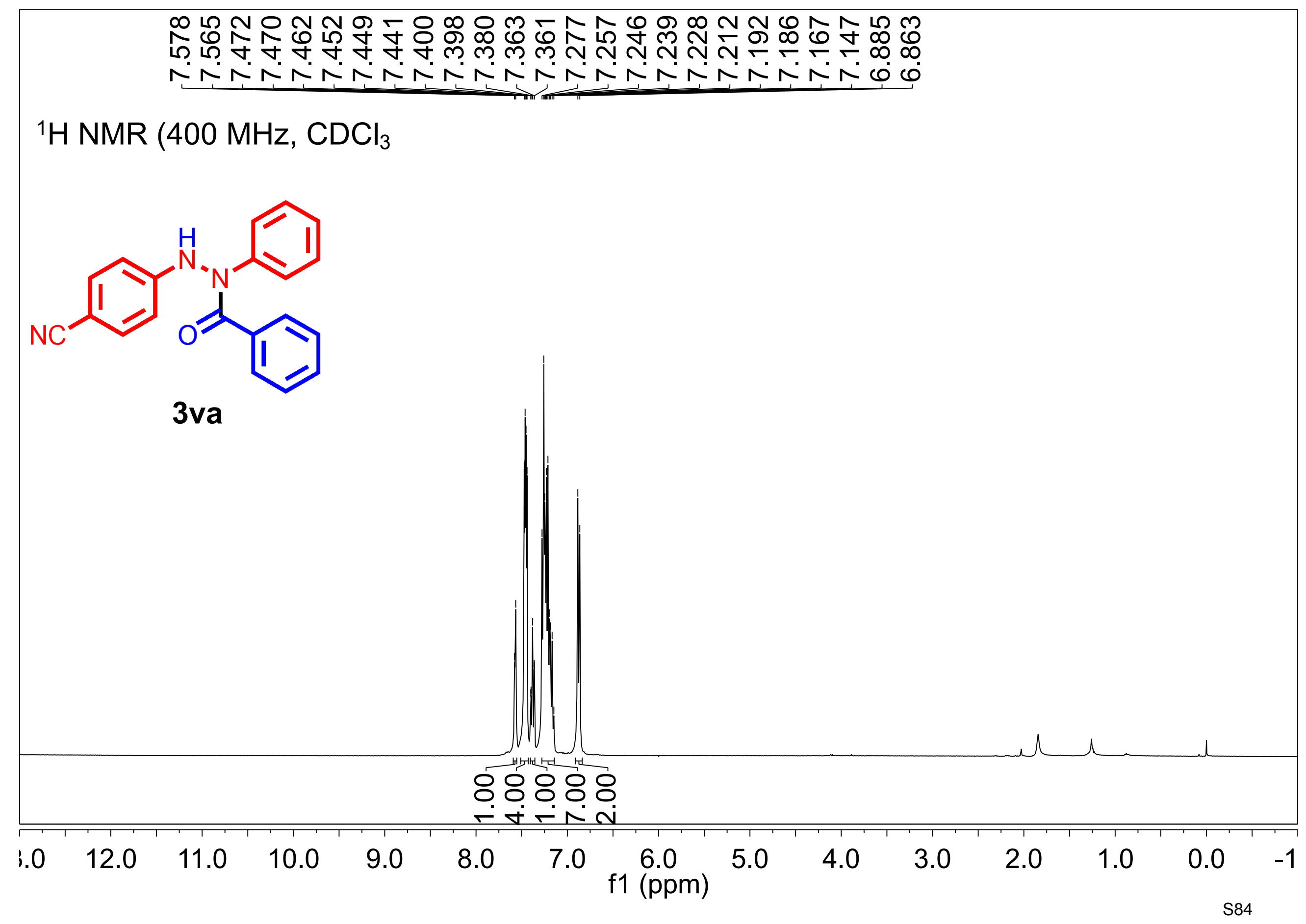




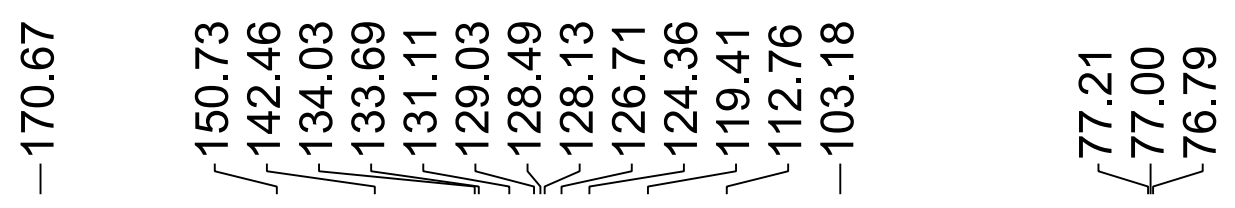

${ }^{13} \mathrm{C}$ NMR (150 MHz, $\left.\mathrm{CDCl}_{3}\right)$

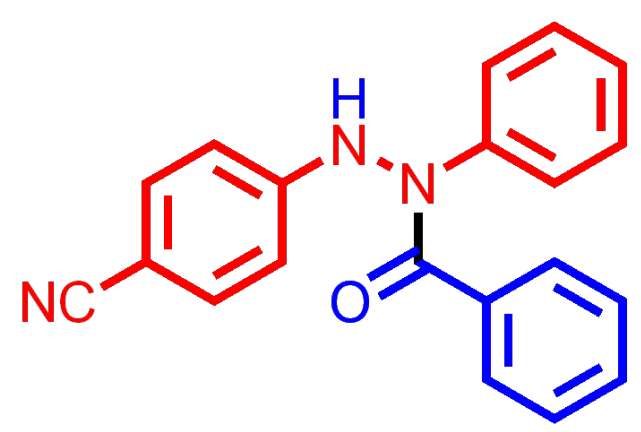

3va

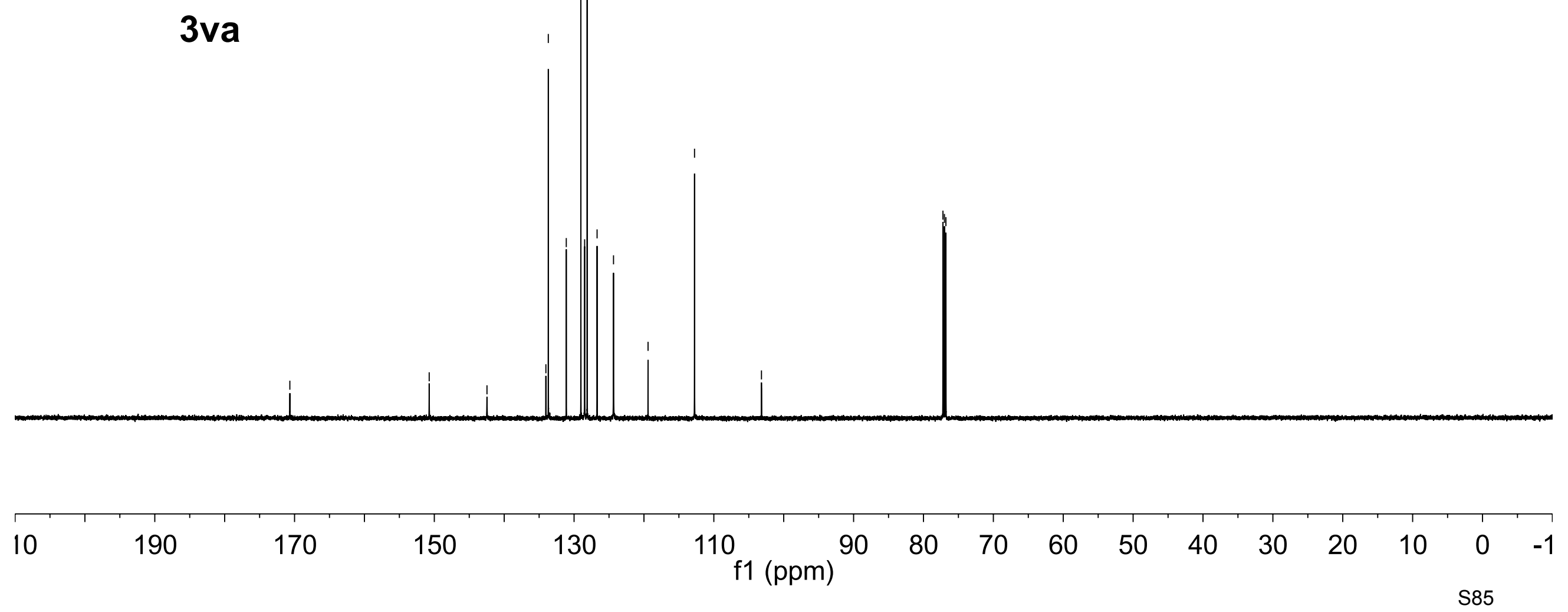


${ }^{1} \mathrm{H}$ NMR $\left(400 \mathrm{MHz}, \mathrm{CDCl}_{3}\right)$

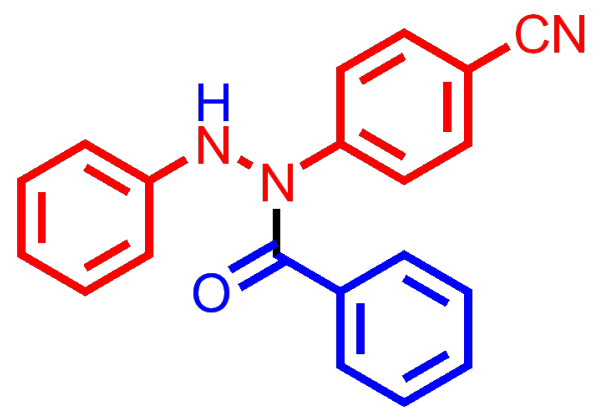

3va'

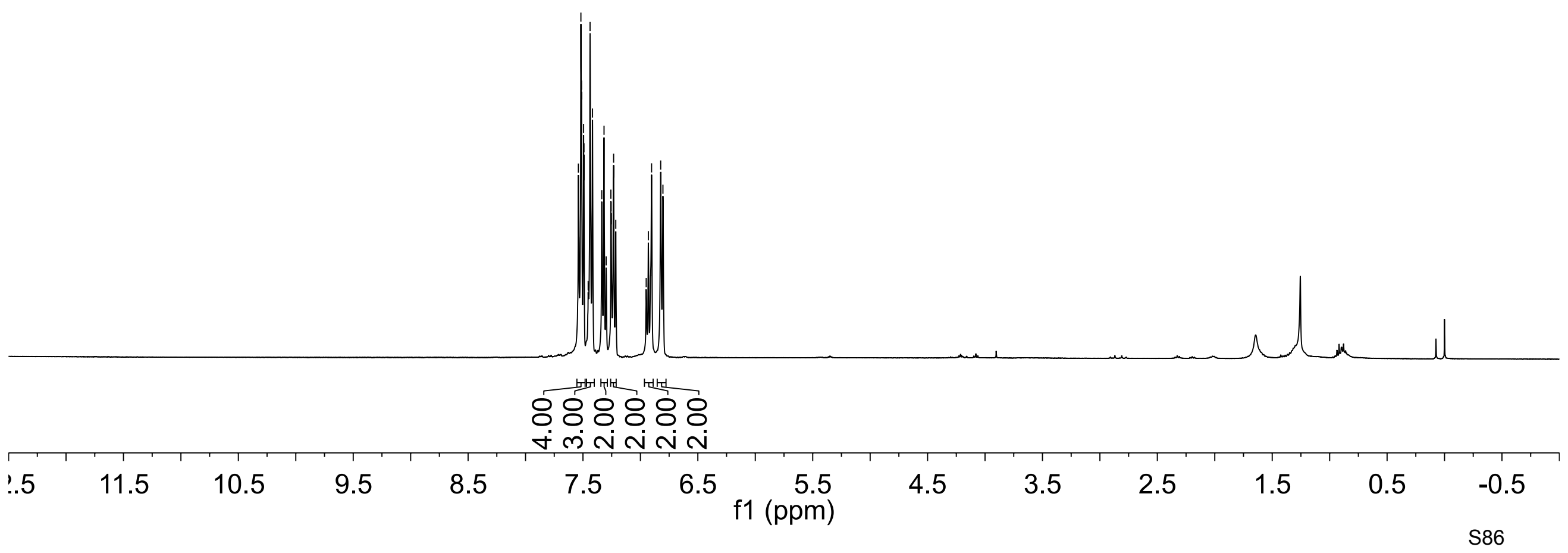


${ }^{13} \mathrm{C} \mathrm{NMR}\left(150 \mathrm{MHz}, \mathrm{CDCl}_{3}\right)$<smiles>N#Cc1ccc(N(Nc2ccccc2)C(=O)c2ccccc2)cc1</smiles>

3va'

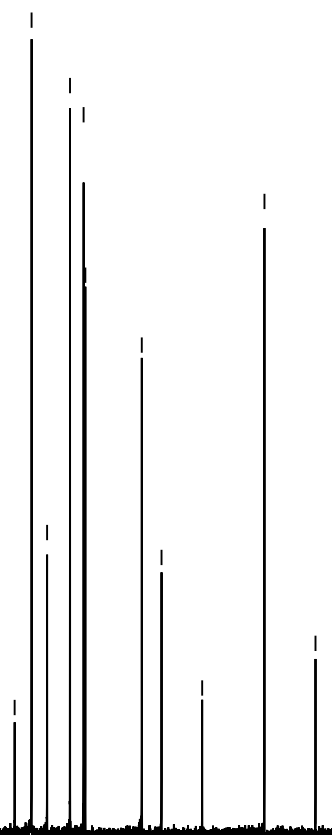




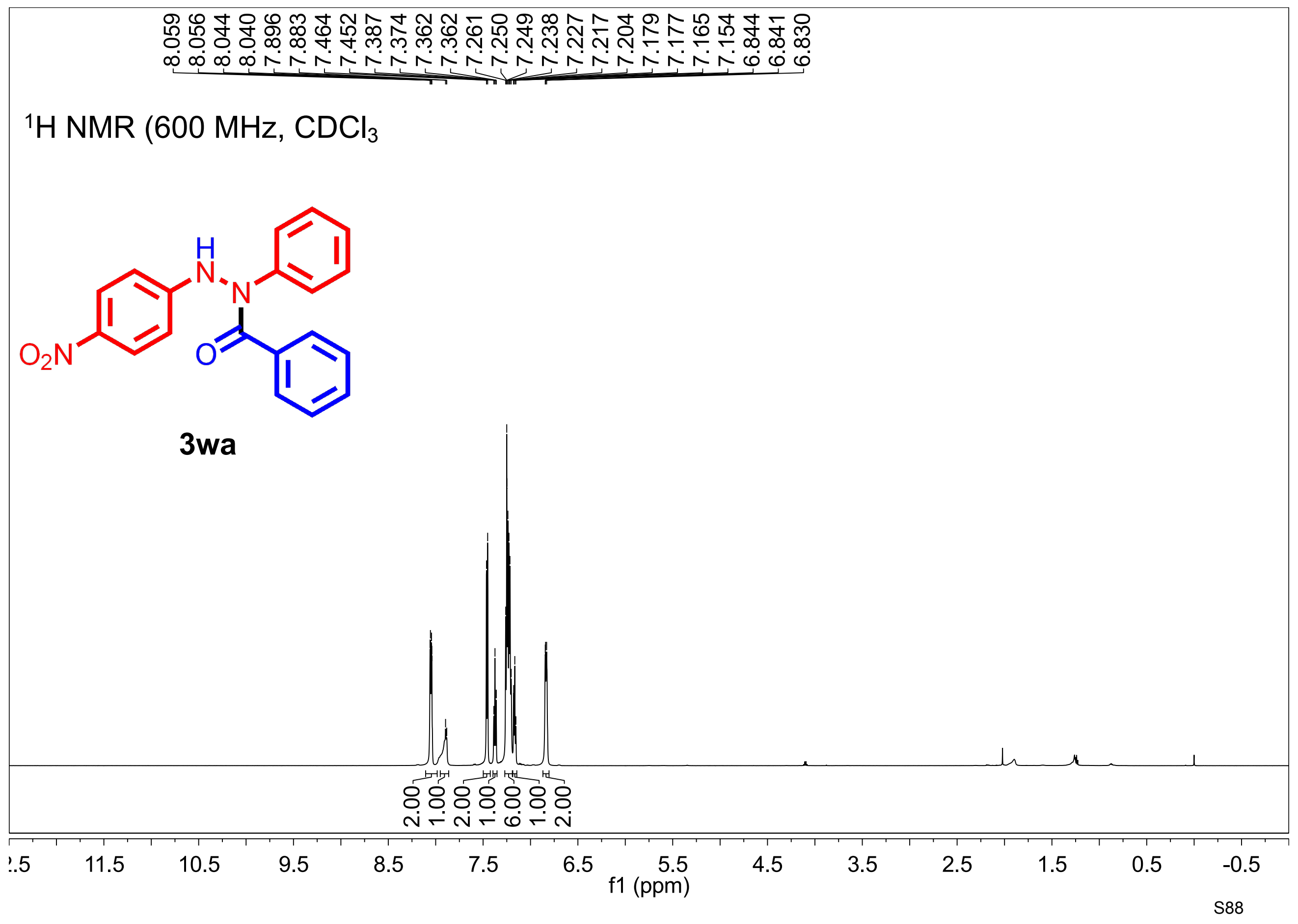




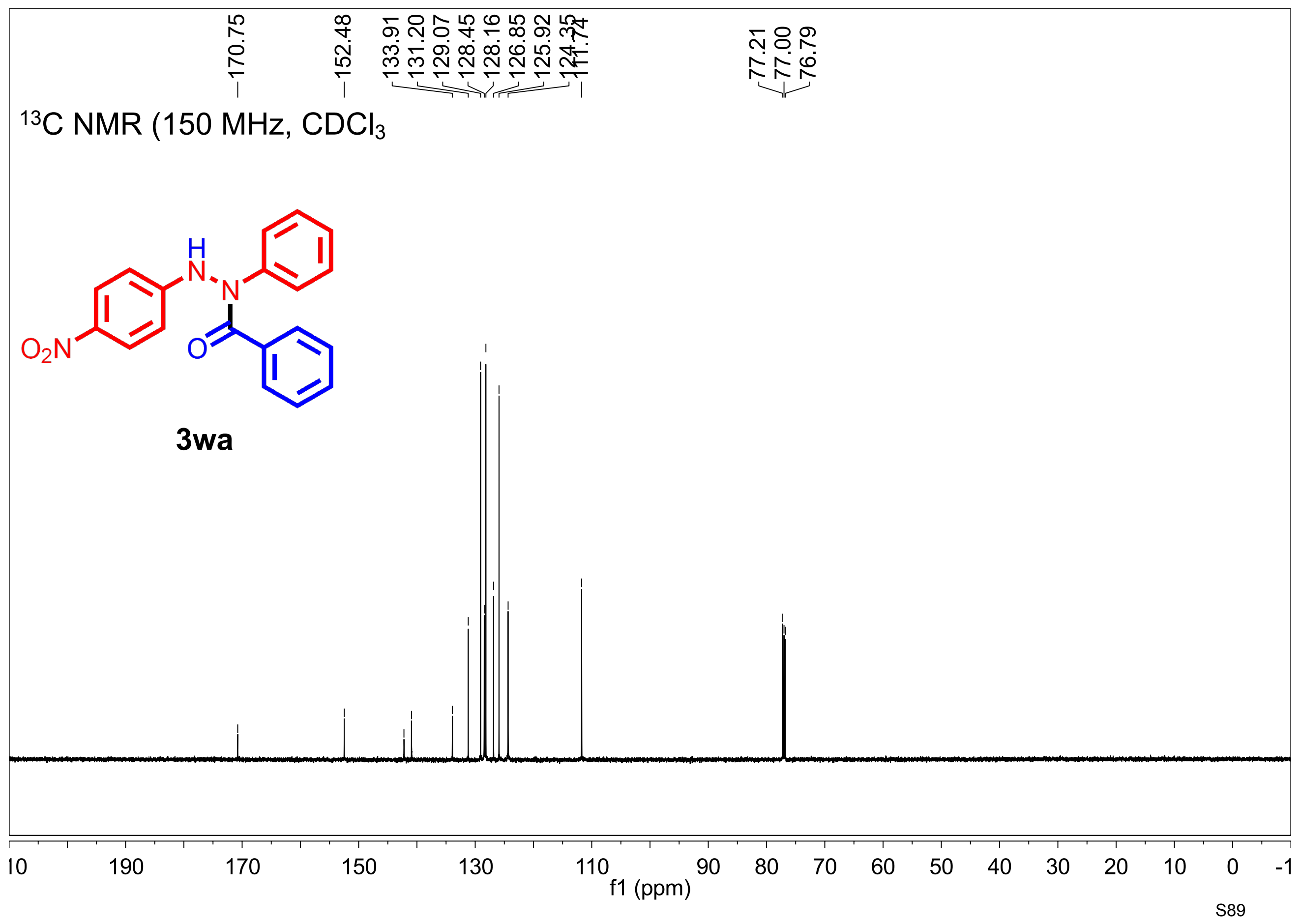




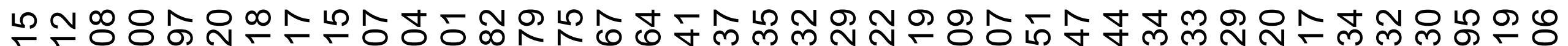
FЕ으음

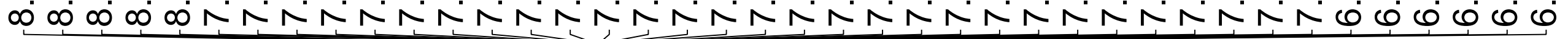

${ }^{1} \mathrm{H}$ NMR $\left(600 \mathrm{MHz}, \mathrm{CDCl}_{3}\right)$

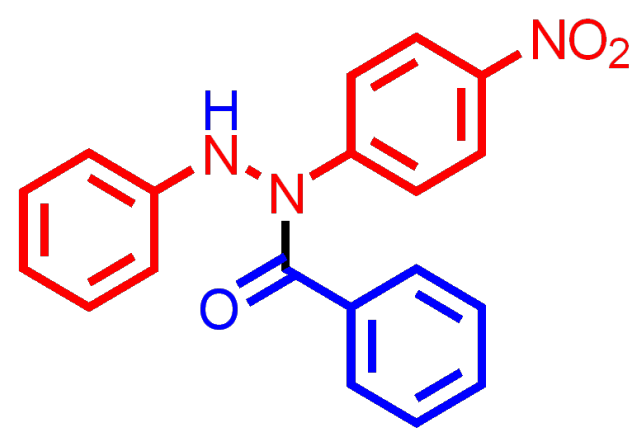

3wa'

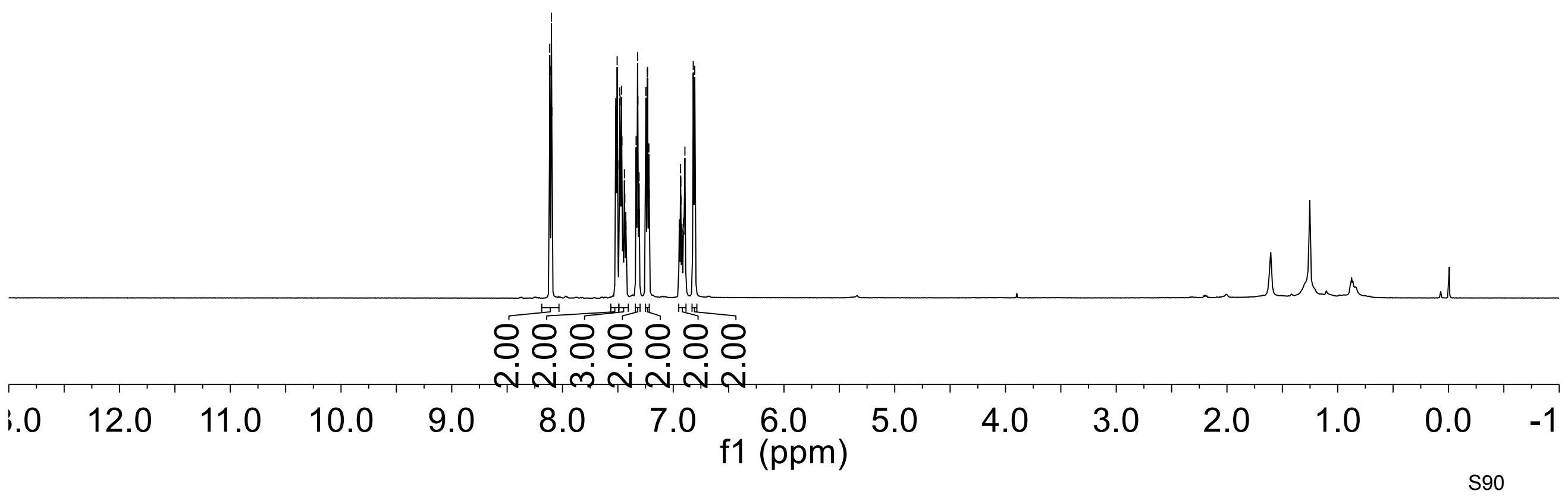




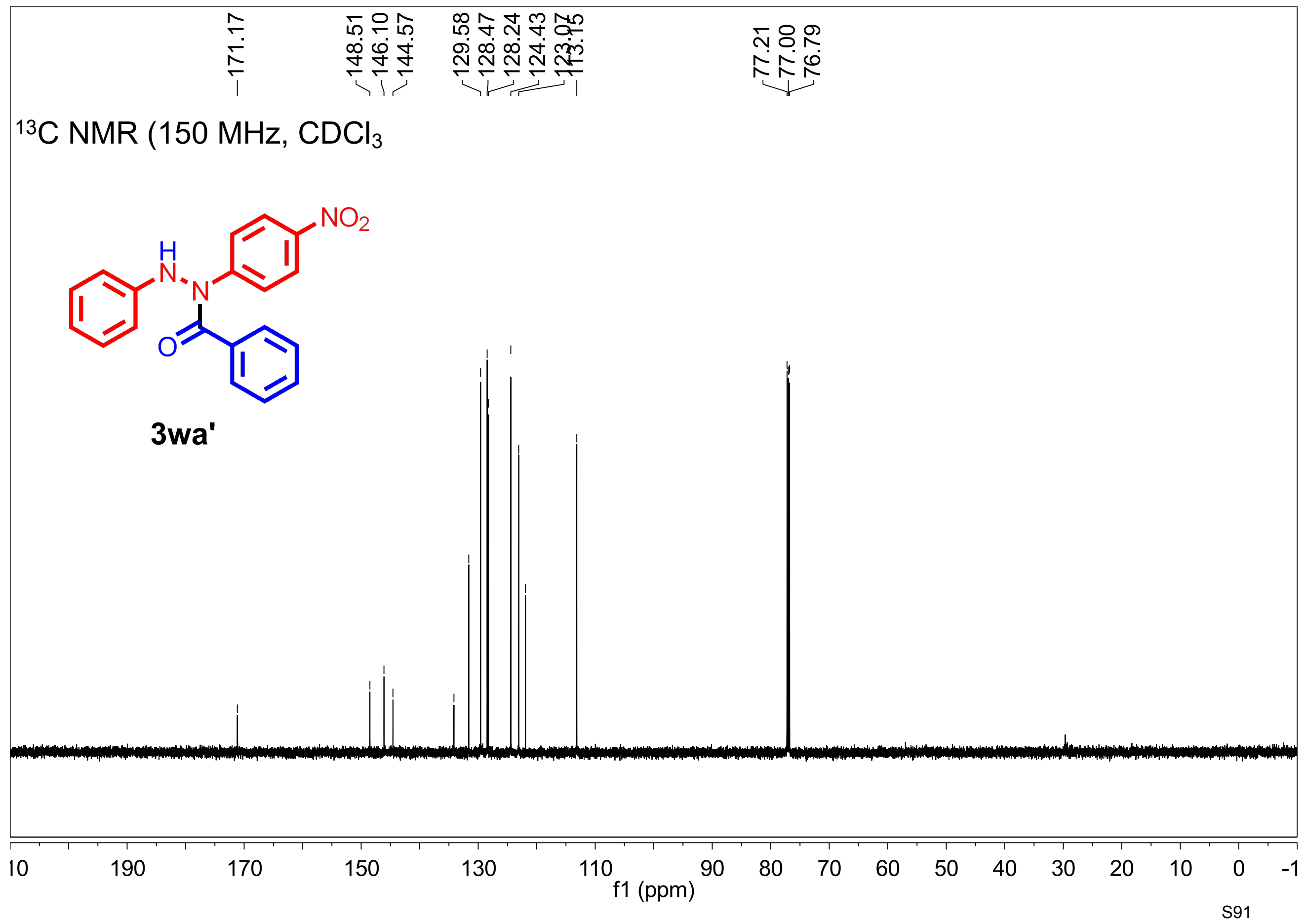


${ }^{1} \mathrm{H}$ NMR $\left(600 \mathrm{MHz}, \mathrm{CDCl}_{3}\right)$

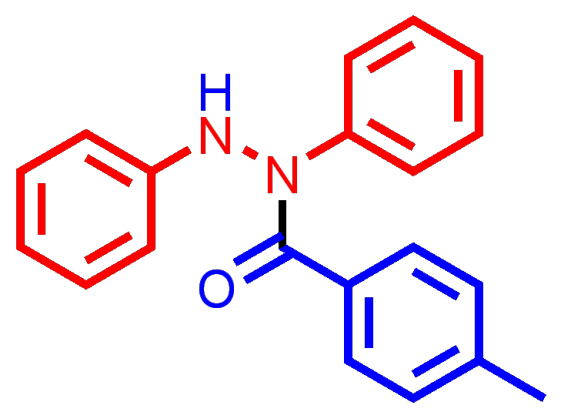

$3 a b$

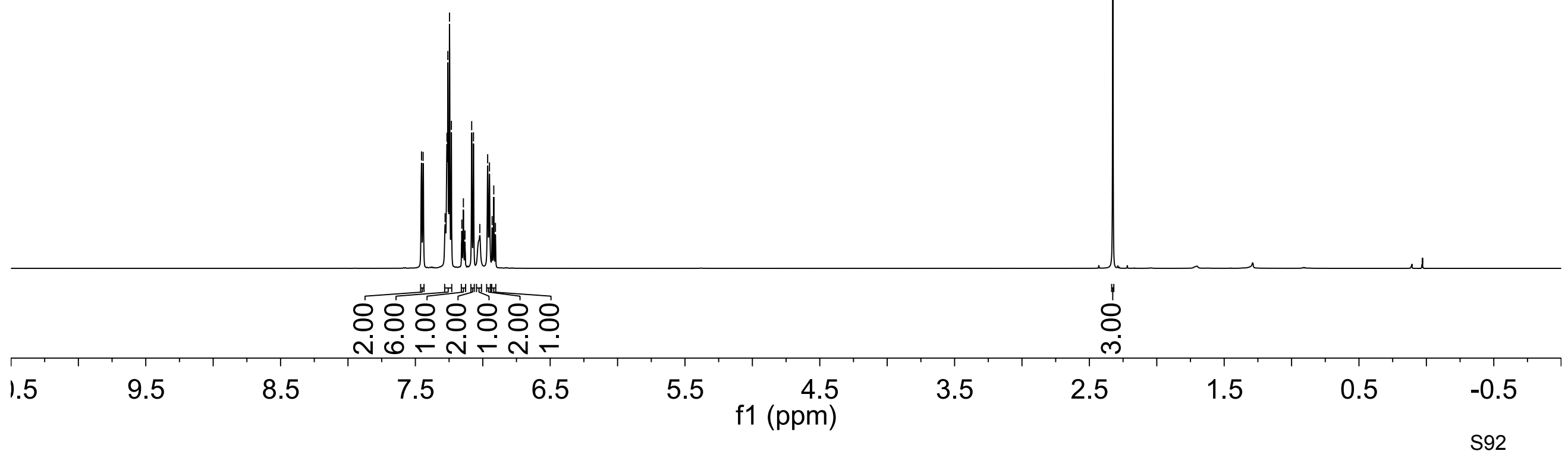




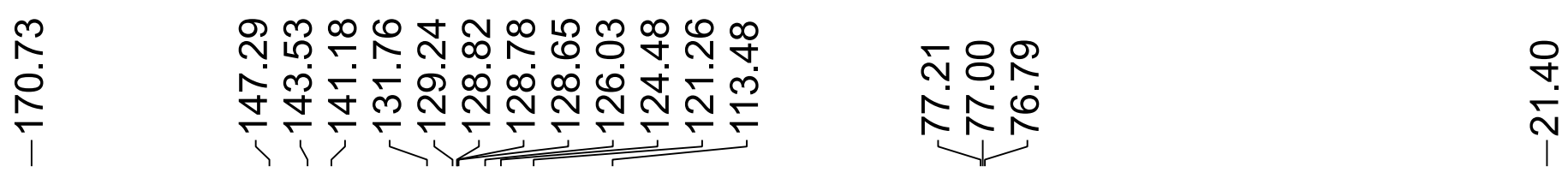

${ }^{13} \mathrm{C}$ NMR $\left(150 \mathrm{MHz}, \mathrm{CDCl}_{3}\right)$<smiles>Cc1ccc(C(=O)N(Nc2ccccc2)c2ccccc2)cc1</smiles>

$3 a b$ 


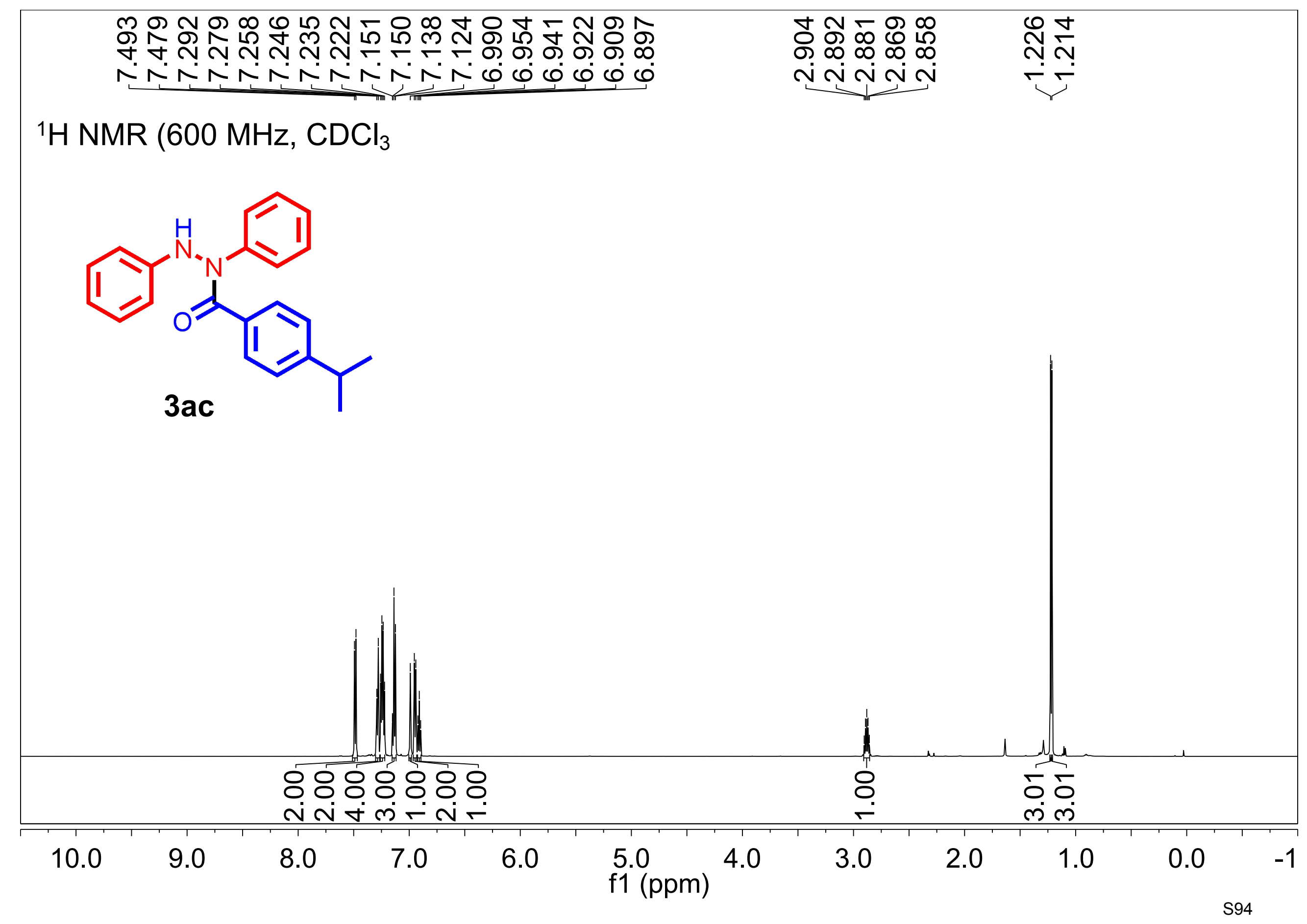




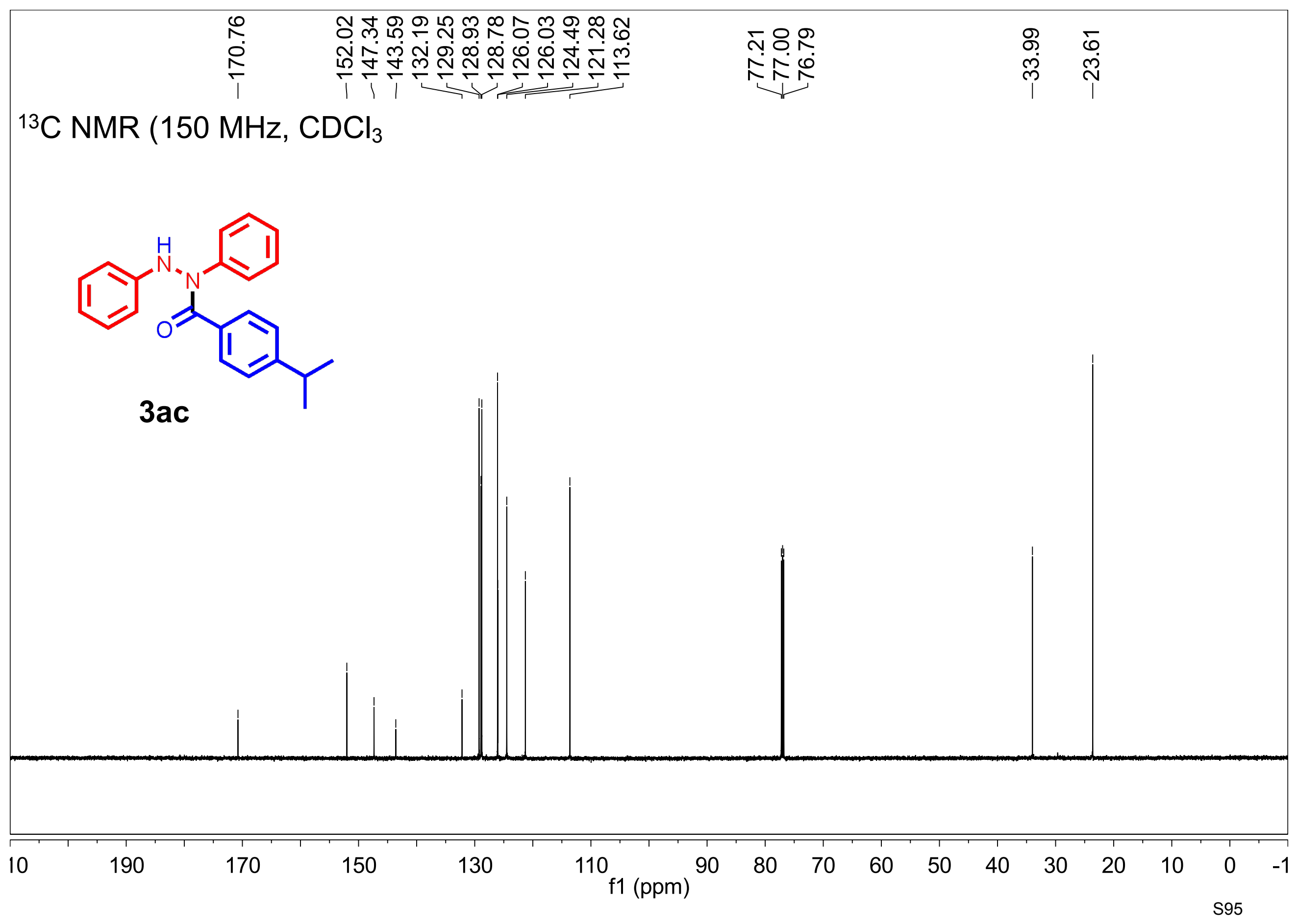




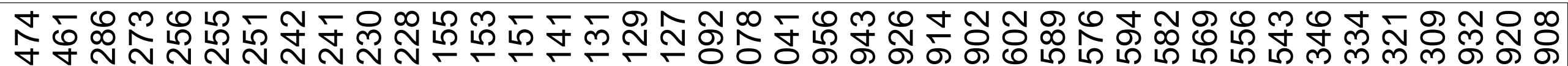

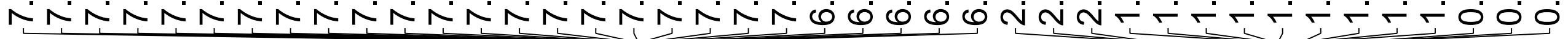

${ }^{1} \mathrm{H}$ NMR $\left(600 \mathrm{MHz} \mathrm{CDCl}_{3}\right)$

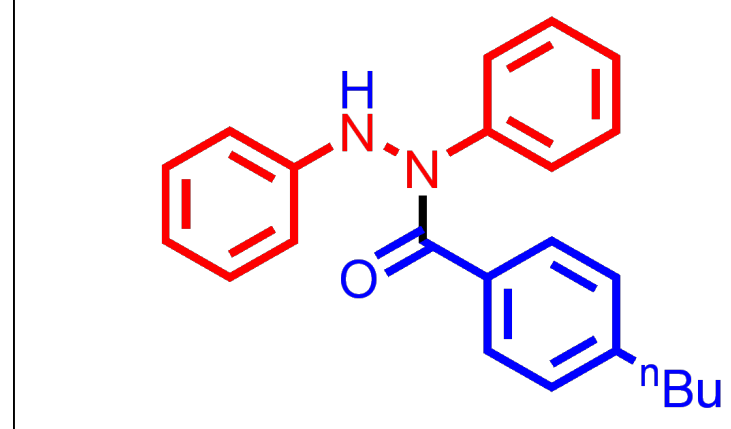

3ad

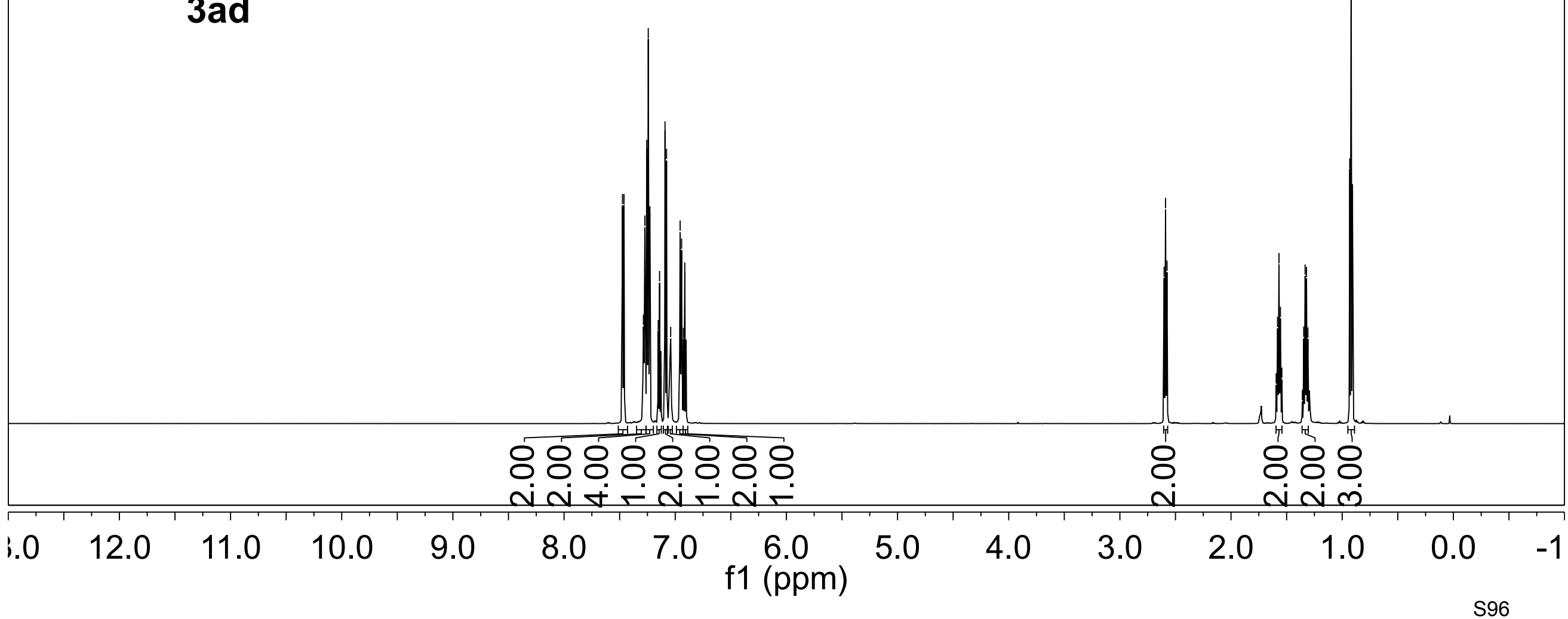




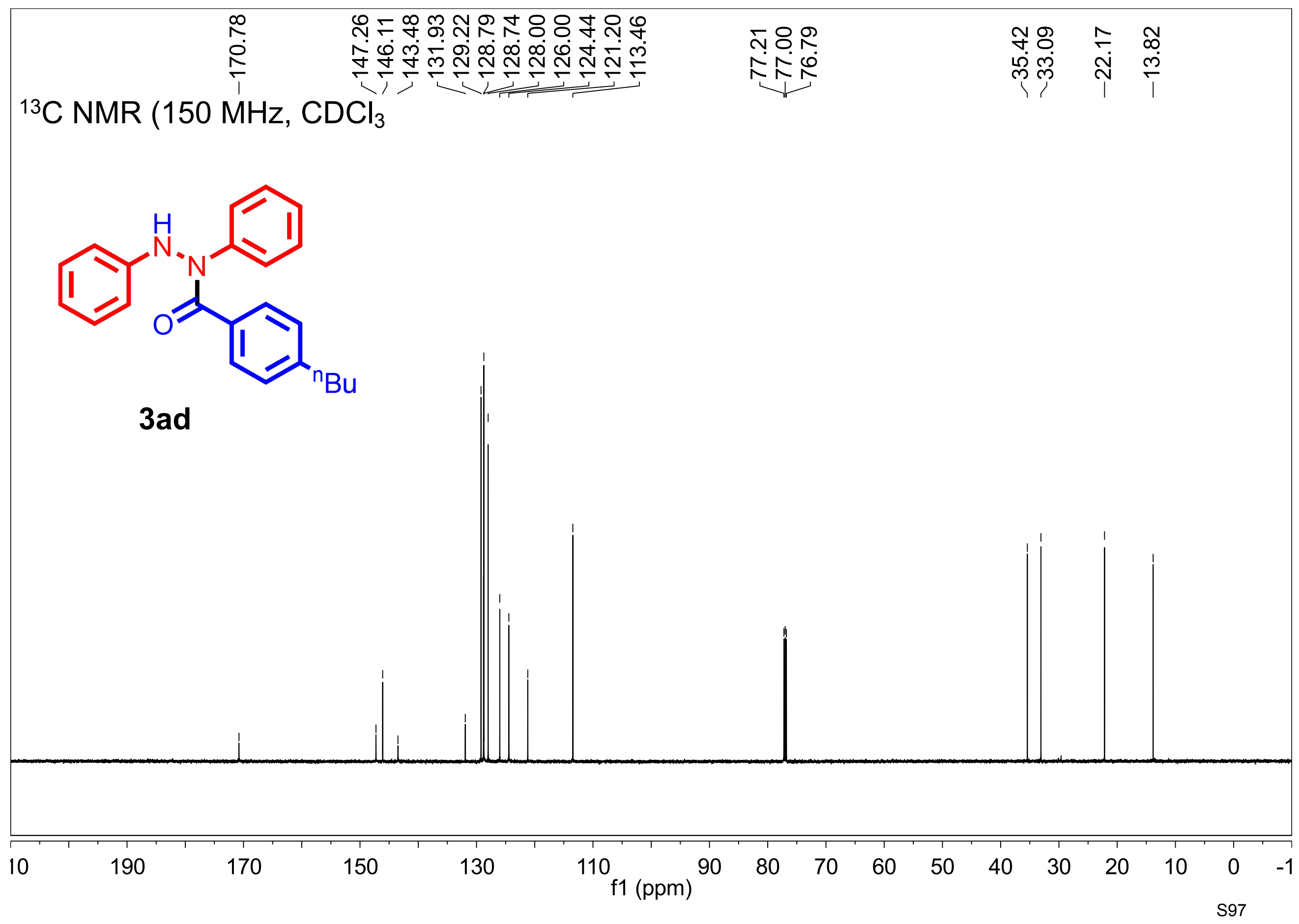




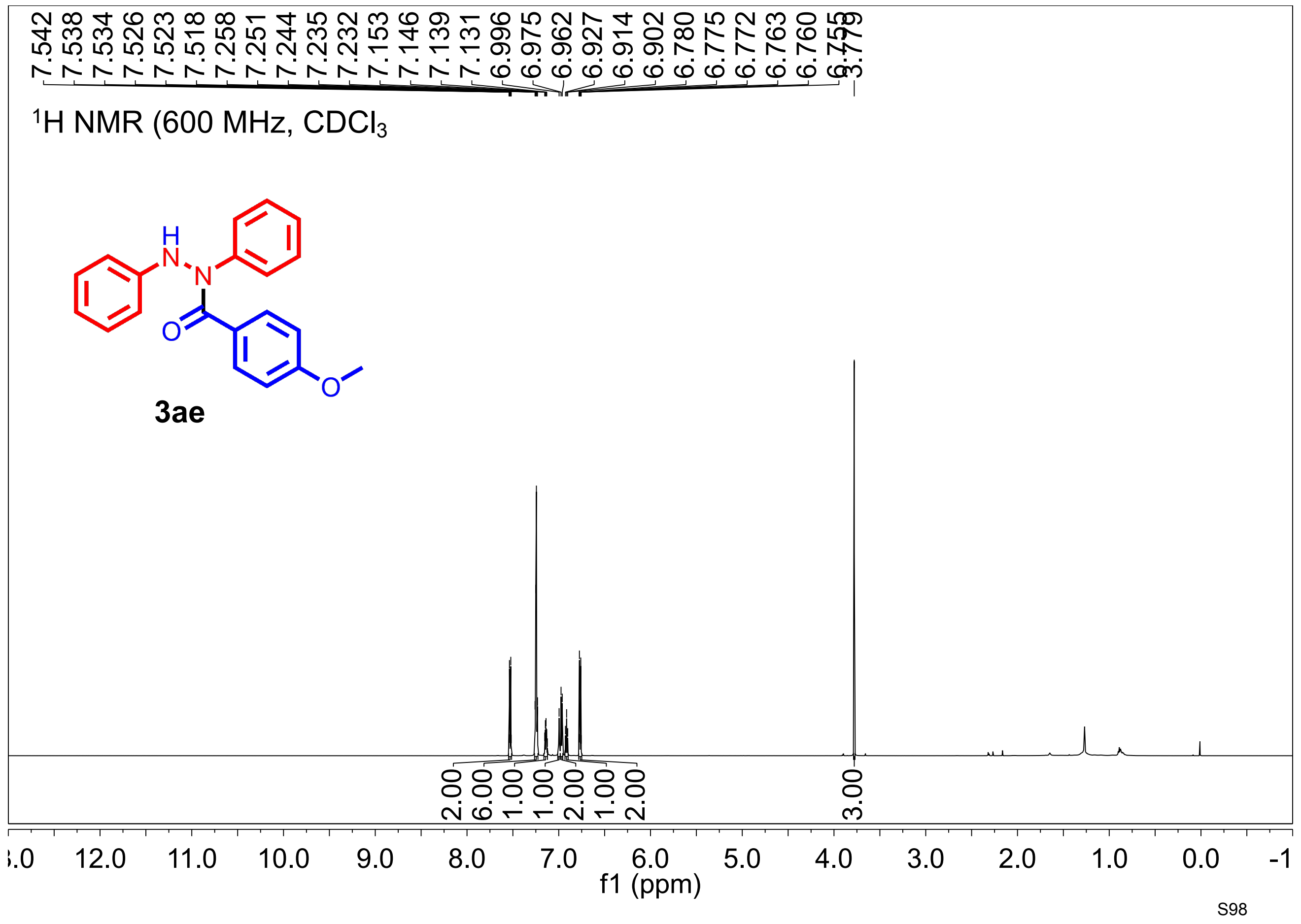




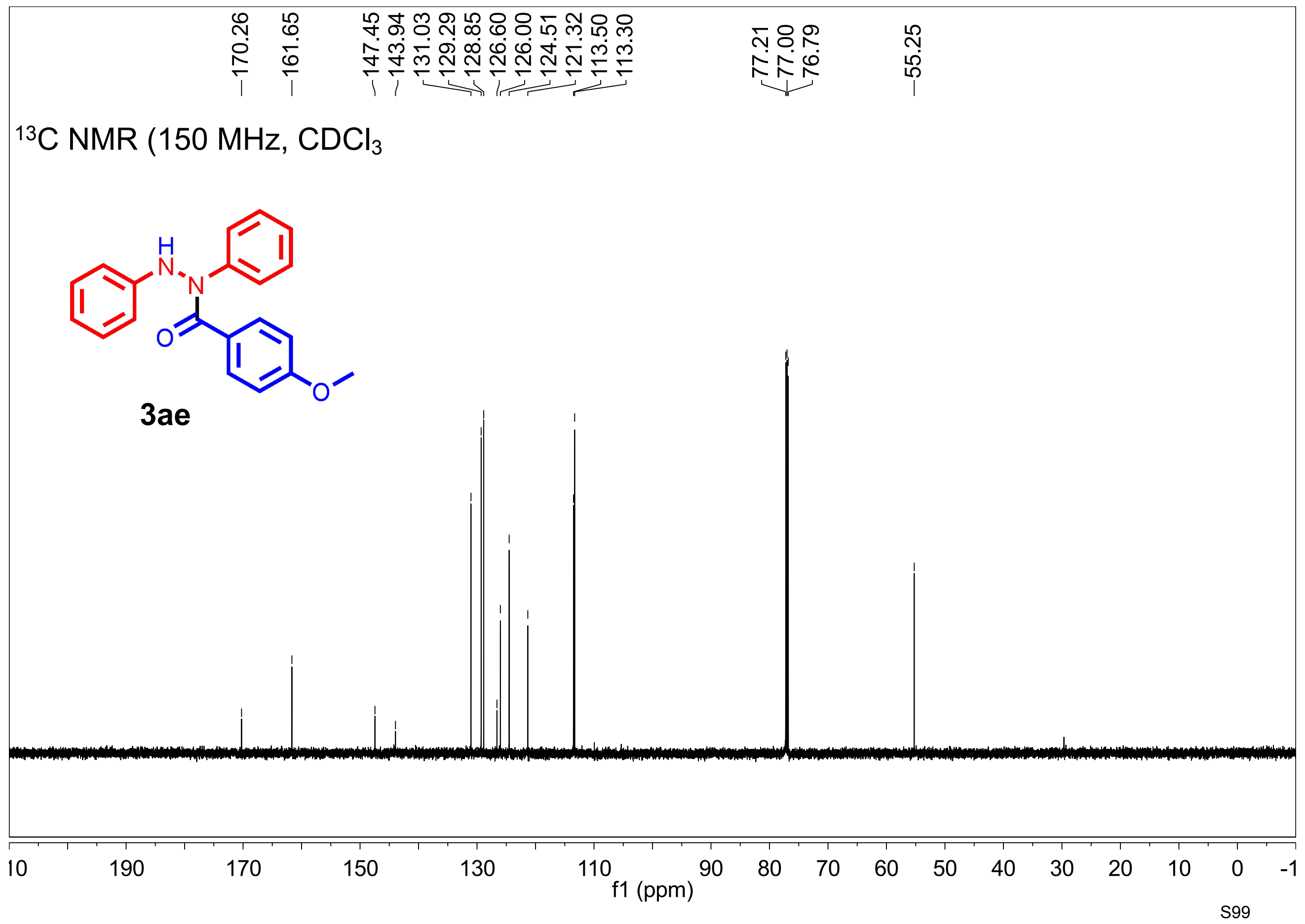


苞 싱 దी

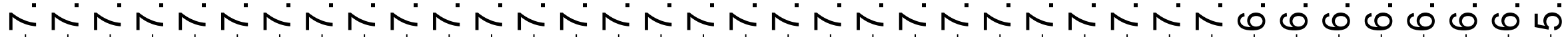

${ }^{1} \mathrm{H}$ NMR $\left(600 \mathrm{MHz}, \mathrm{CDCl}_{3}\right)$

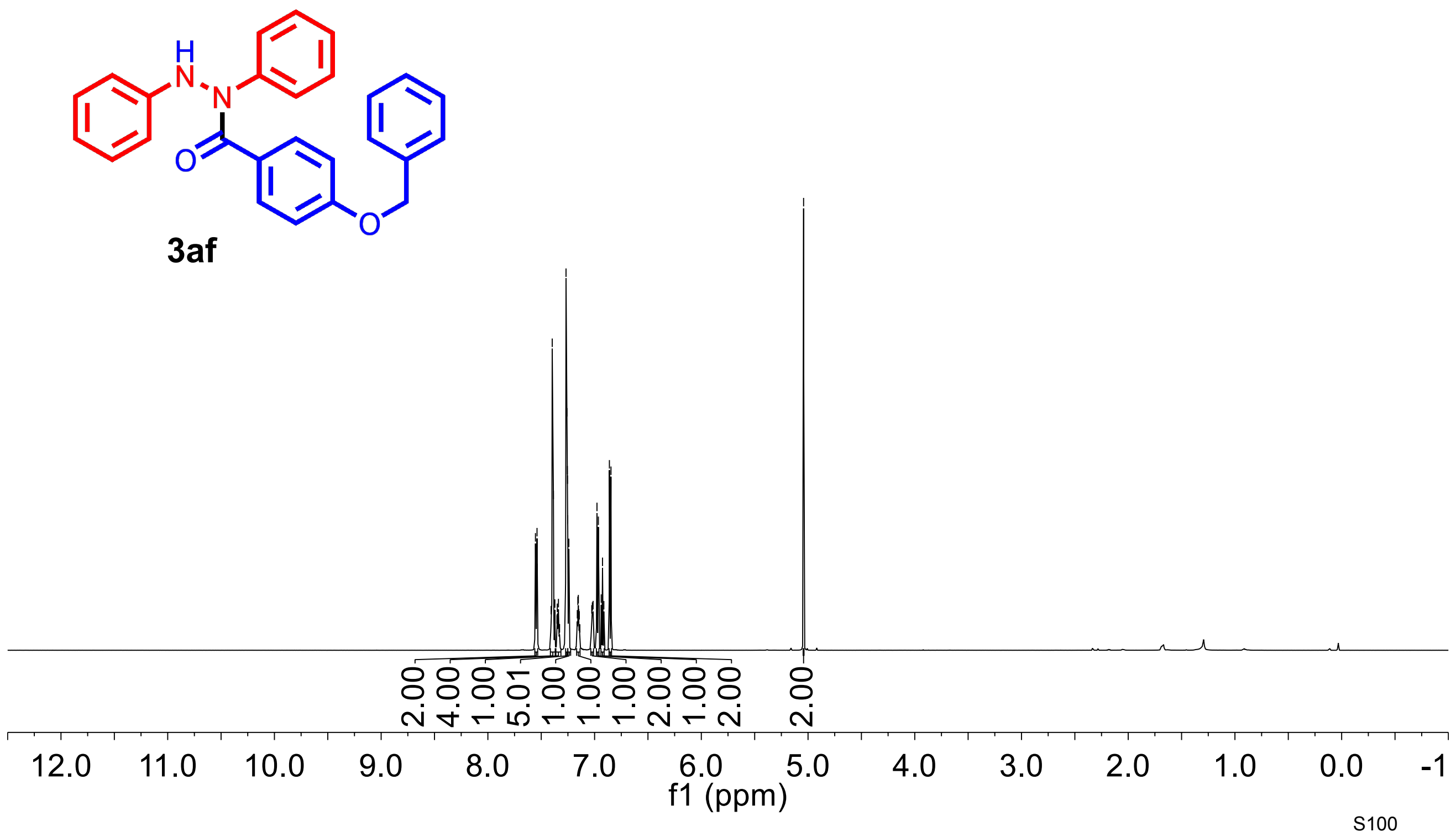




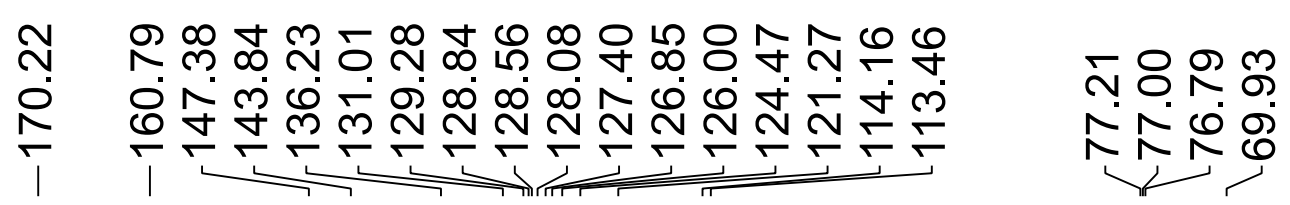

${ }^{13} \mathrm{C}$ NMR $\left(150 \mathrm{MHz}, \mathrm{CDCl}_{3}\right)$

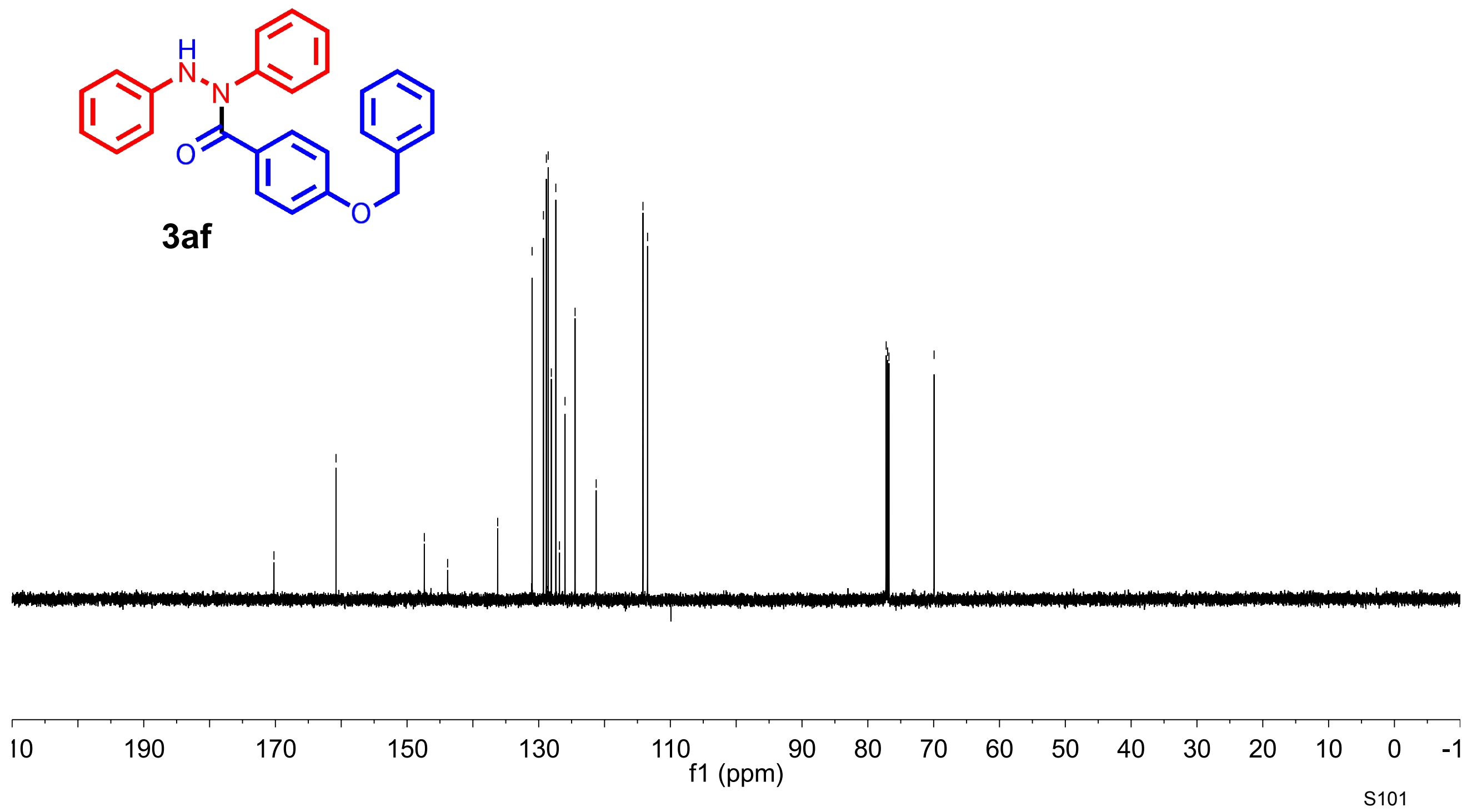




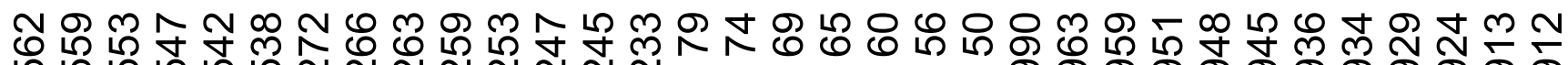

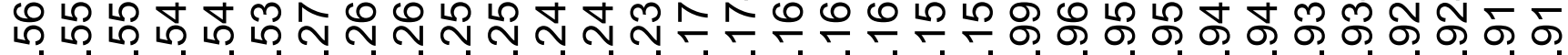
N N N N N N N N N N

${ }^{1} \mathrm{H}$ NMR $\left(600 \mathrm{MHz}, \mathrm{CDCl}_{3}\right)$

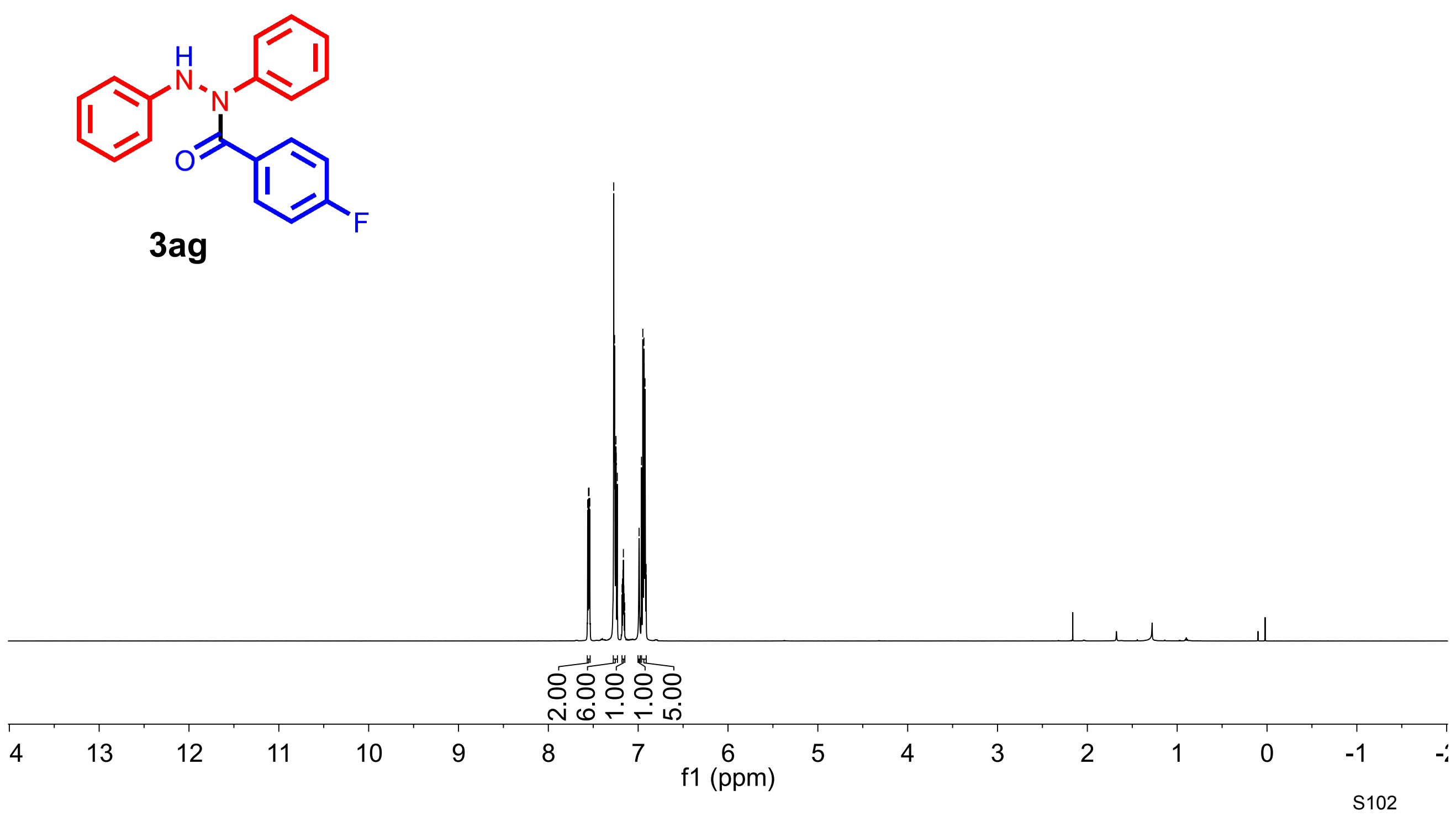




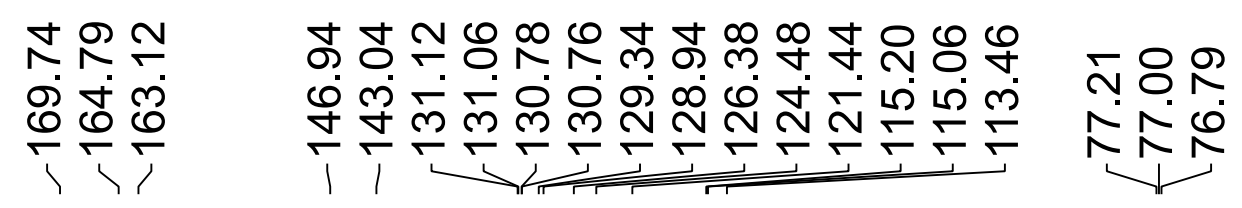

${ }^{13} \mathrm{C}$ NMR $\left(150 \mathrm{MHz}, \mathrm{CDCl}_{3}\right)$

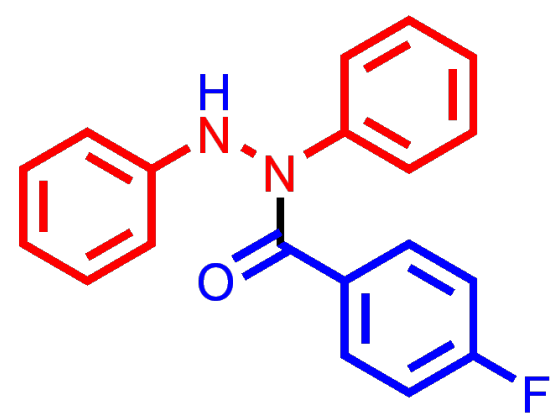

3ag

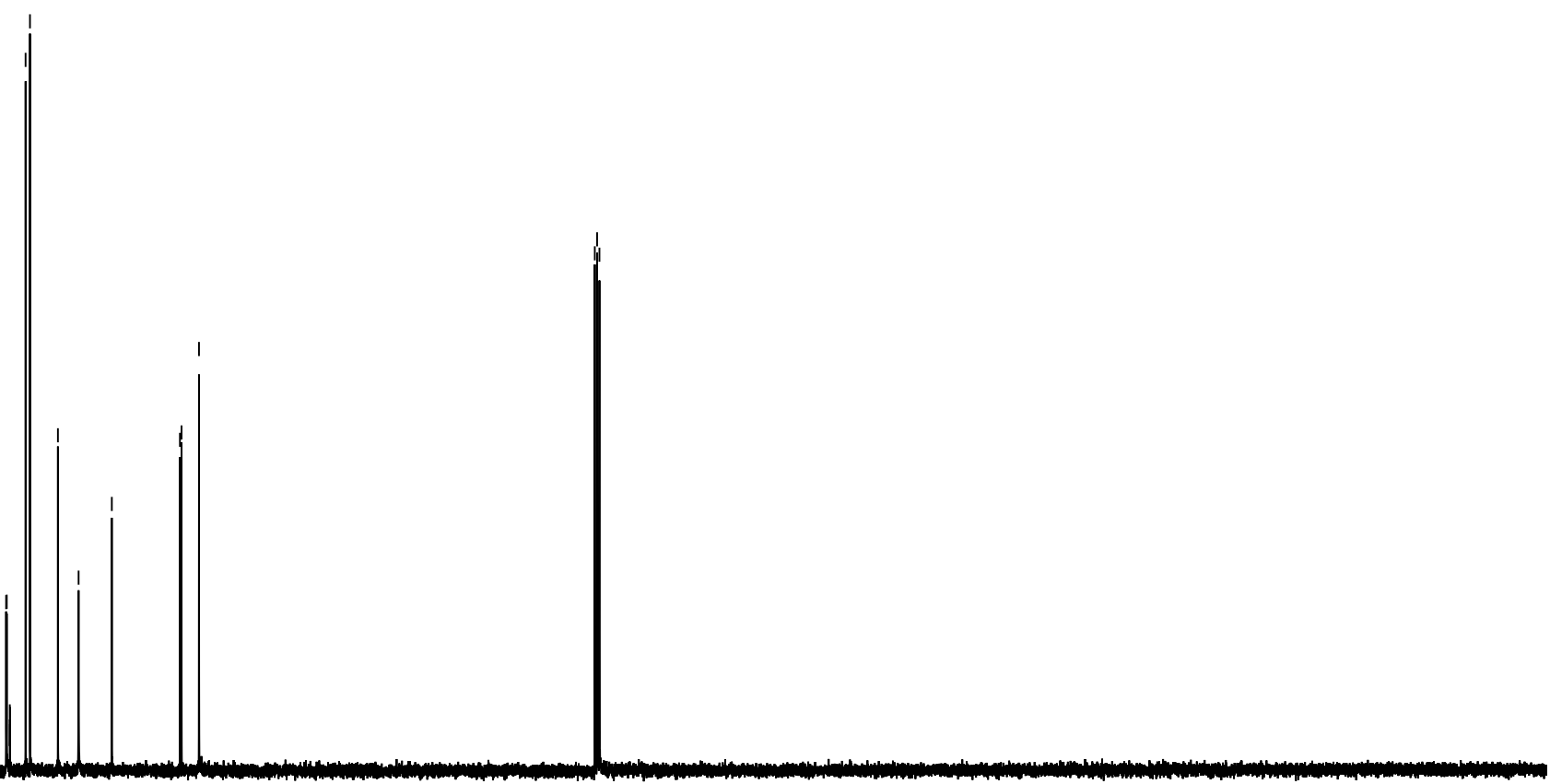




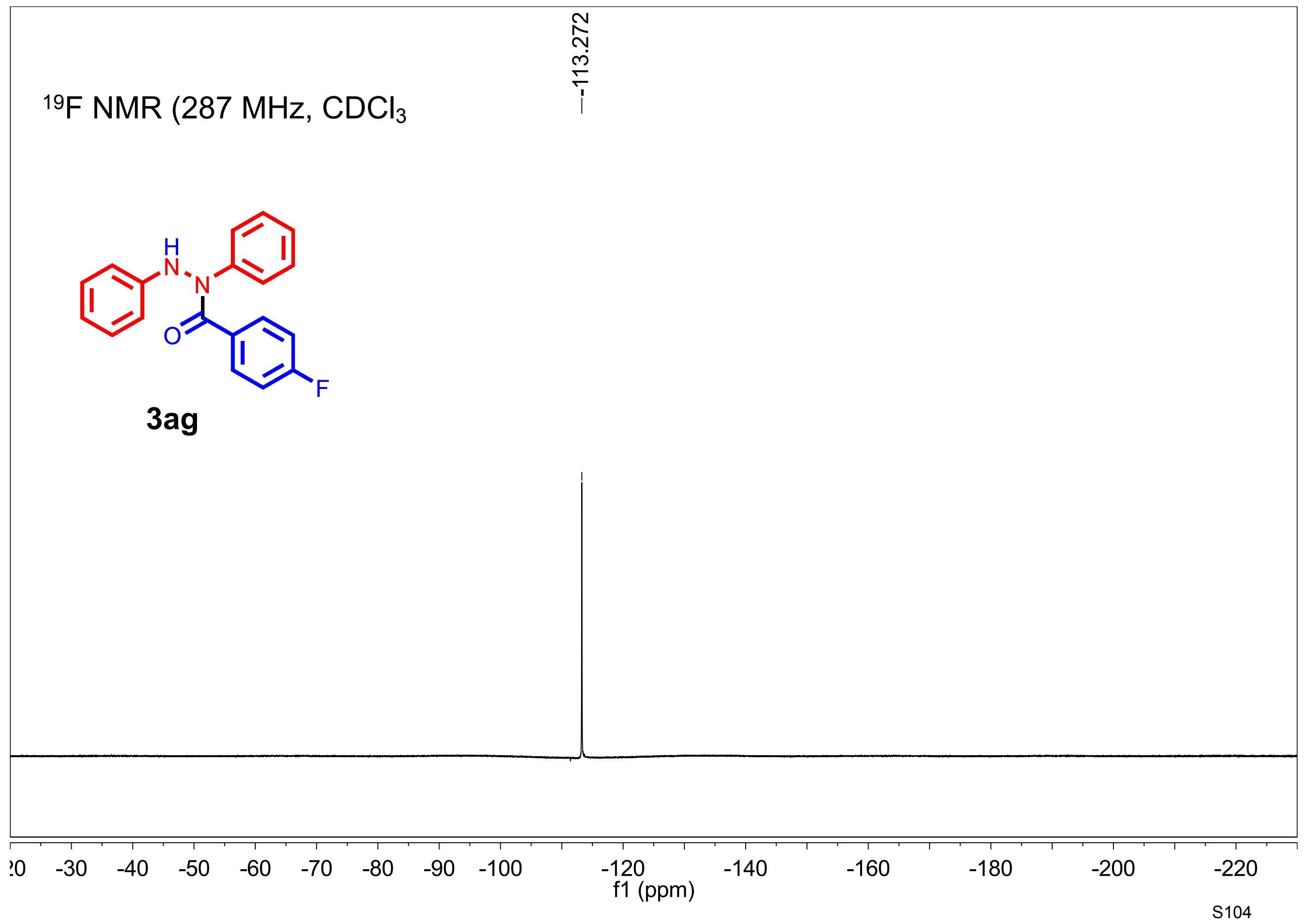


๗

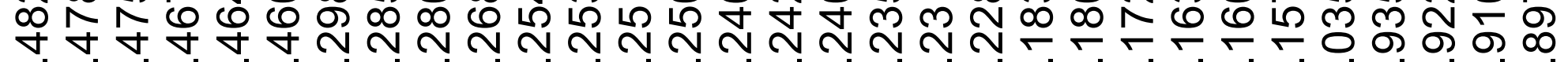

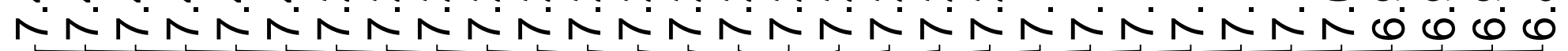

${ }^{1} \mathrm{H}$ NMR $\left(600 \mathrm{MHz}, \mathrm{CDCl}_{3}\right)$

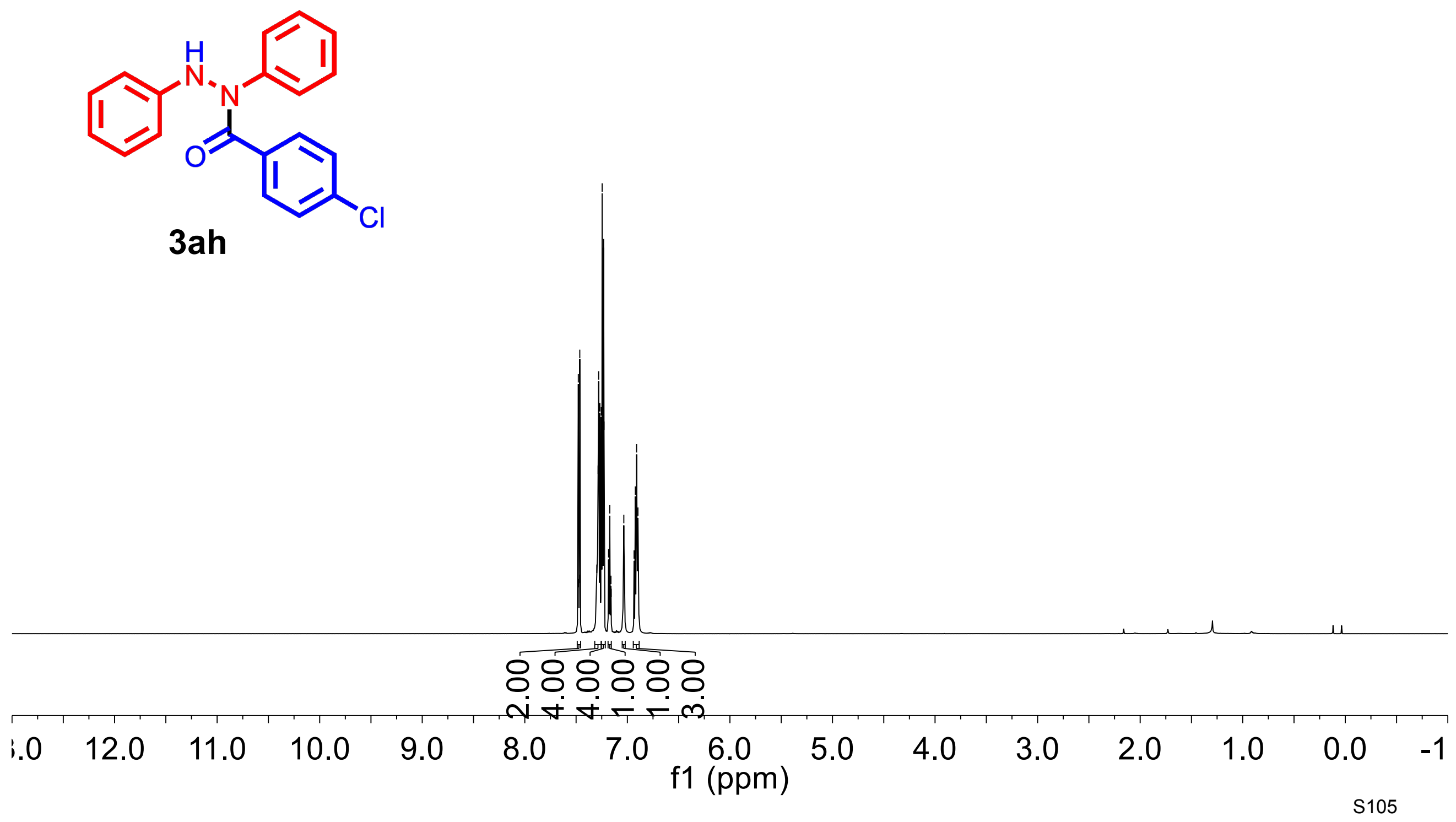




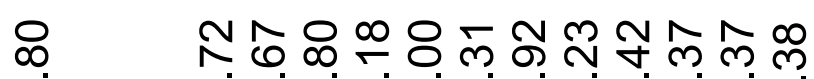

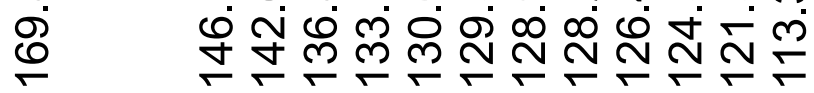

సุำ

กิ่อ

।

Nis

${ }^{13} \mathrm{C}$ NMR (150 MHz, $\mathrm{CDCl}_{3}$ )<smiles>O=C(c1ccc(Cl)cc1)N(Nc1ccccc1)c1ccccc1</smiles>

3ah

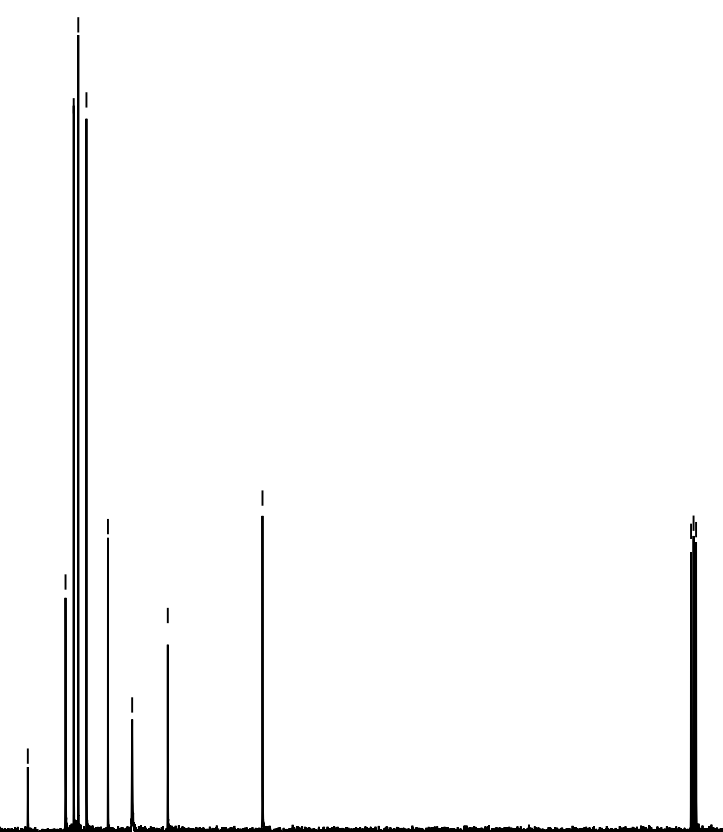




\section{${ }^{1} \mathrm{H}$ NMR $\left(600 \mathrm{MHz}, \mathrm{CDCl}_{3}\right)$}

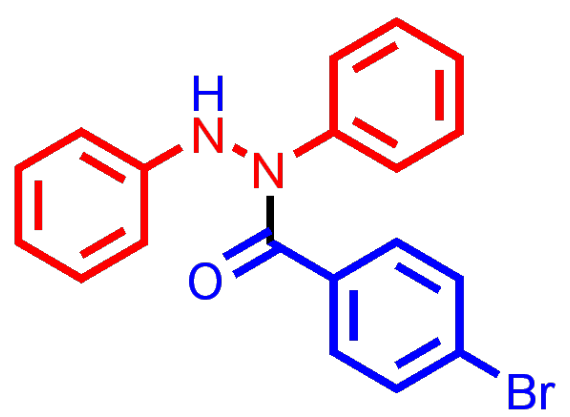

3ai

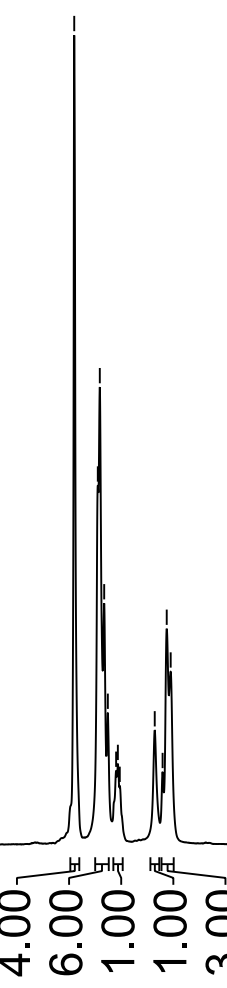

$\begin{array}{llllllll}. .5 & 11.5 & 10.5 & 9.5 & 8.5 & 7.5 & 6.5 & \begin{array}{r}5.5 \\ \mathrm{f} 1(\mathrm{ppm})\end{array}\end{array}$

4.5

3.5

2.5

1.5

0.5

$-0.5$ 


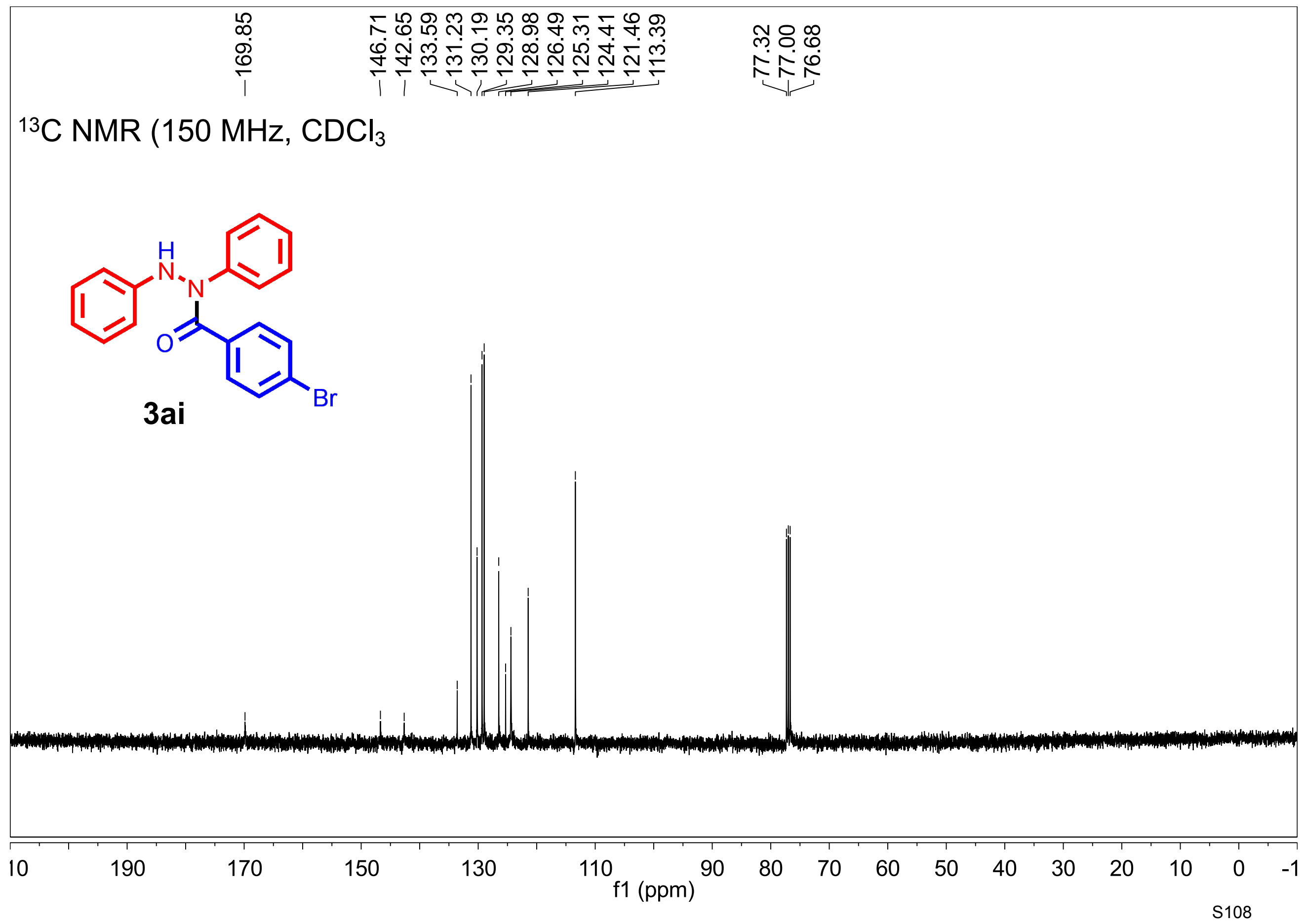




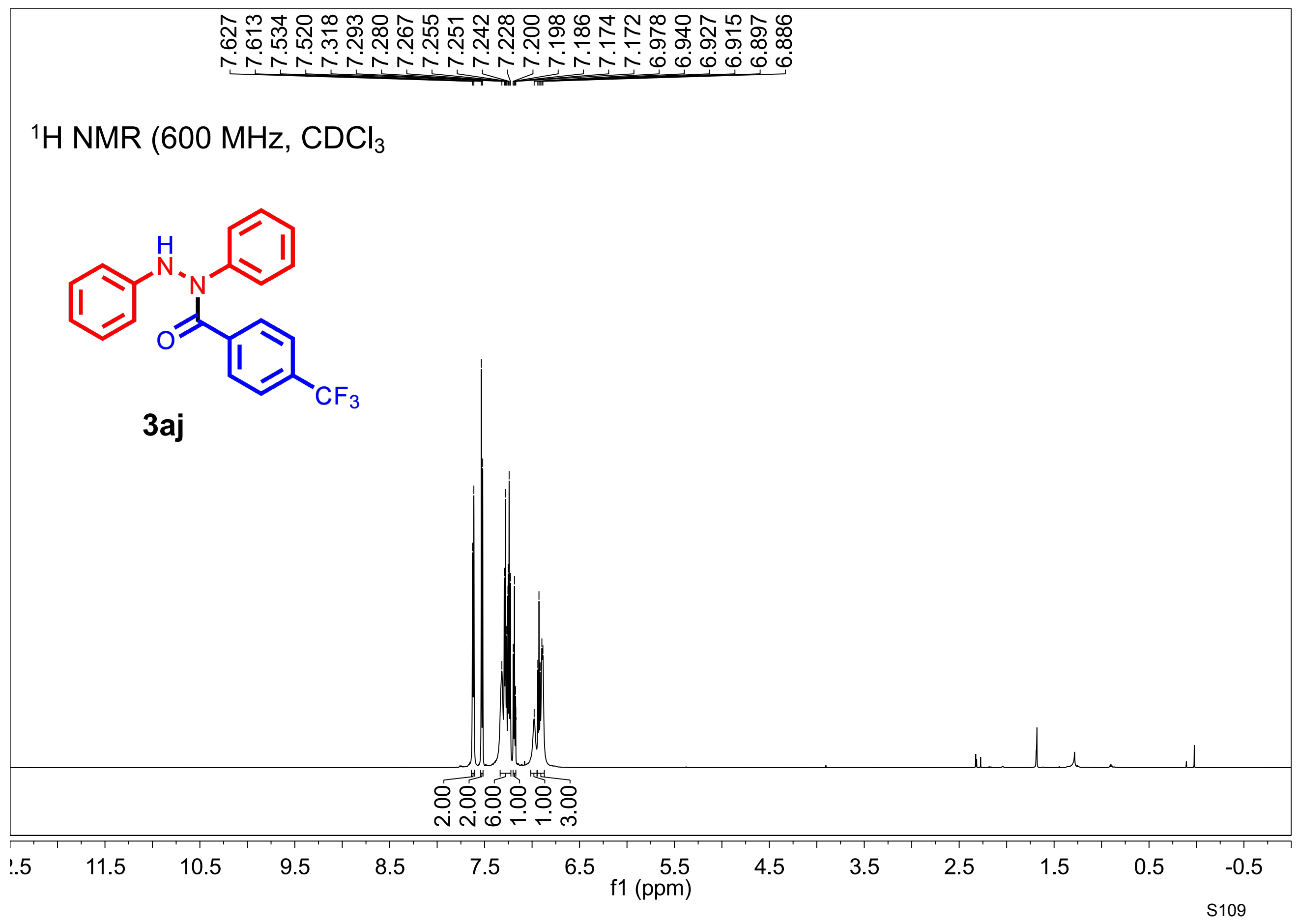


の ஸ

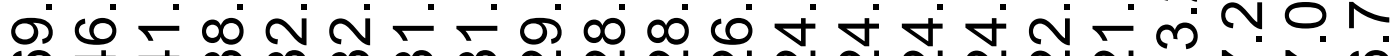

GIVM

${ }^{13} \mathrm{C}$ NMR $\left(150 \mathrm{MHz}, \mathrm{CDCl}_{3}\right)$

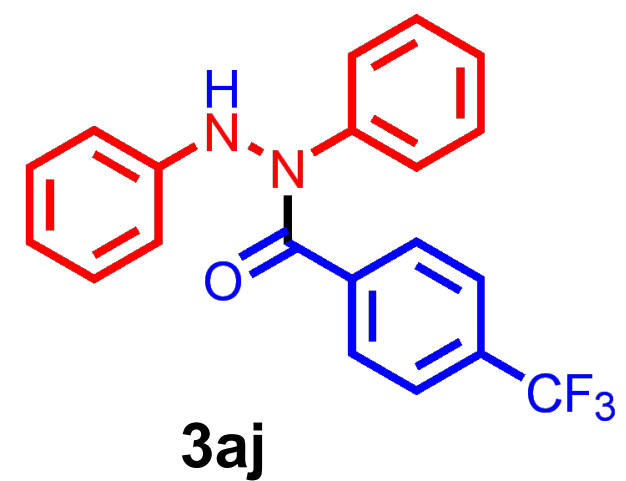

$\begin{array}{lllll}30 & 210 \quad 190 \quad 170 \quad 150 \quad 130 & 110 \\ \text { f1 } & (\text { ppm })\end{array}$

70

70

50

30

$10-1$ 
${ }^{19} \mathrm{~F}$ NMR $\left(287 \mathrm{MHz}, \mathrm{CDCl}_{3}\right)$

\section{F NMR (287 MHz, $\left.\mathrm{CDCl}_{3}\right)$}

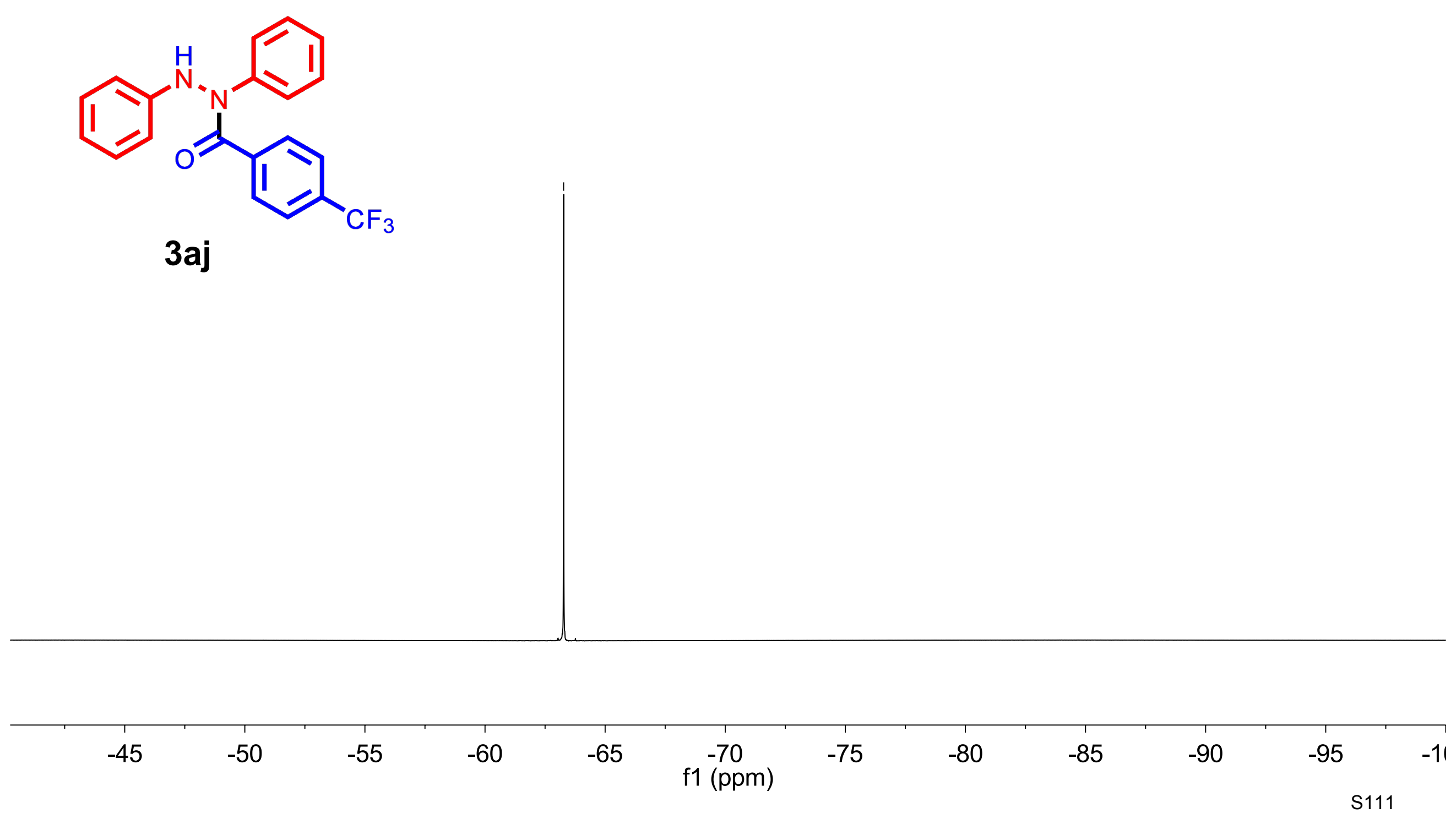




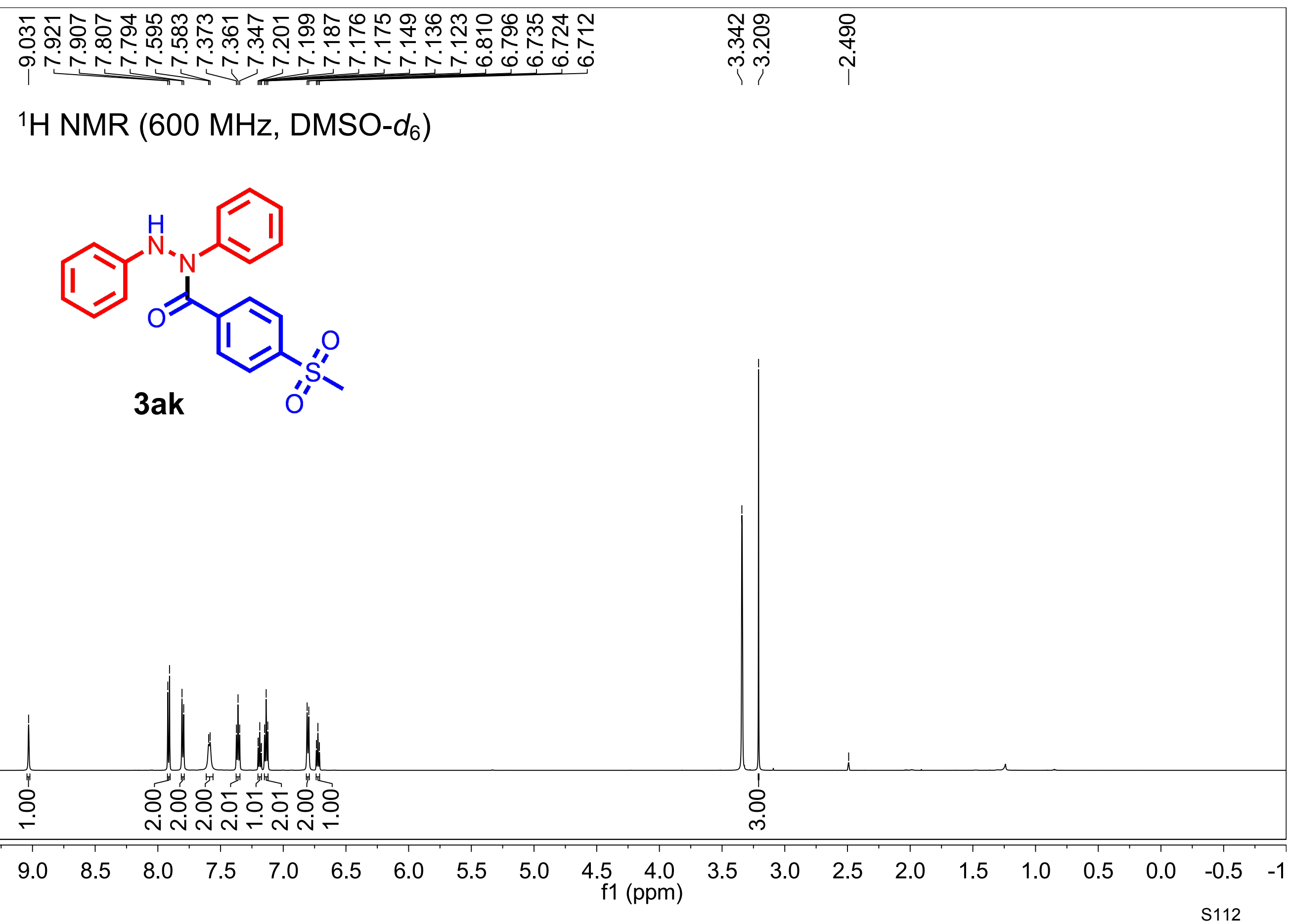




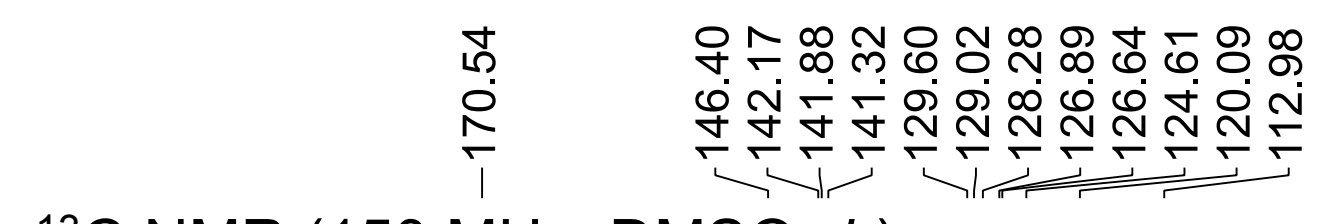

${ }^{13} \mathrm{C}$ NMR (150 MHz, DMSO- $d_{6}$ )

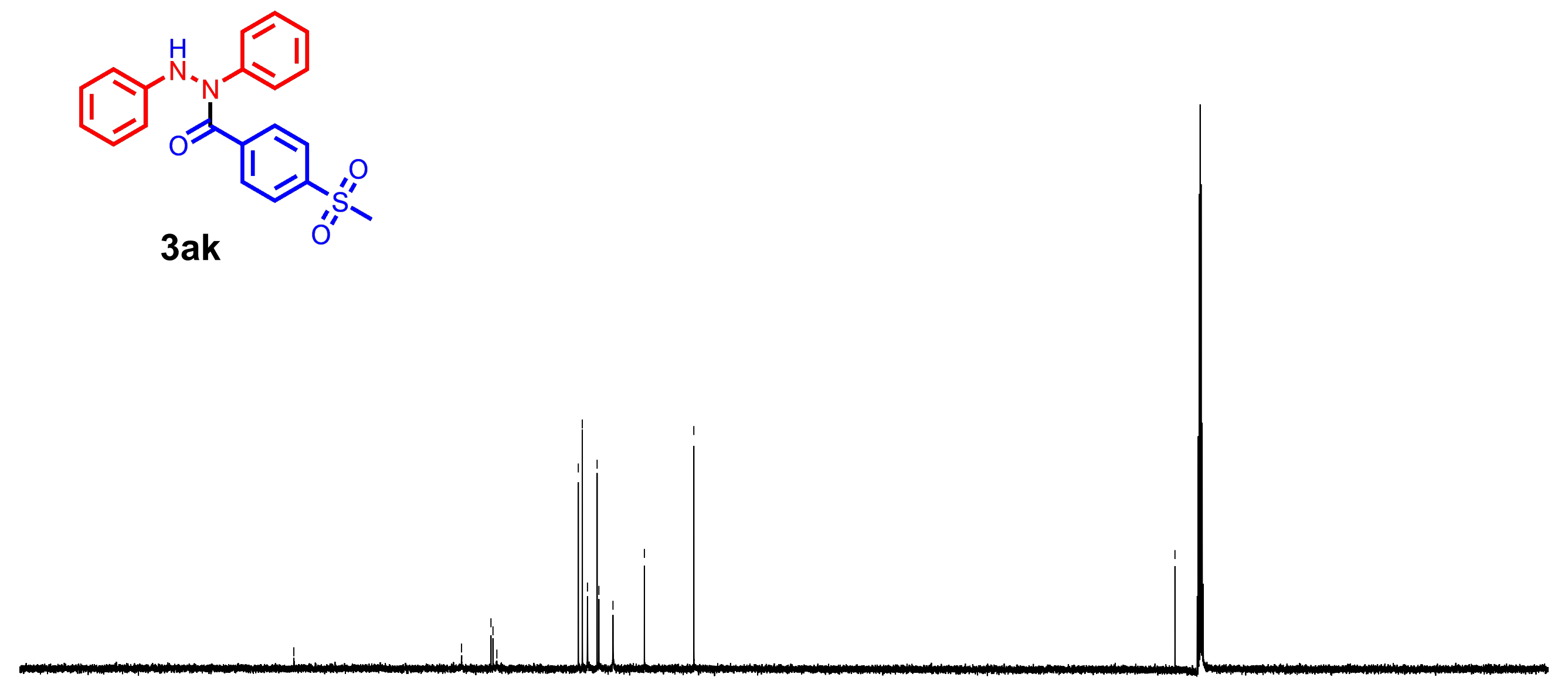

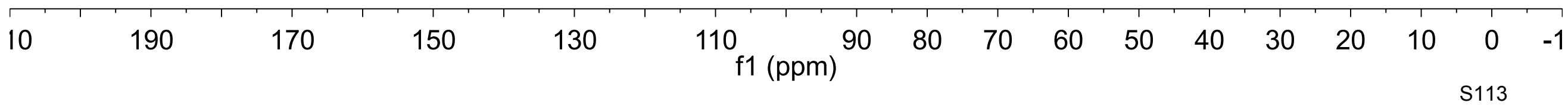




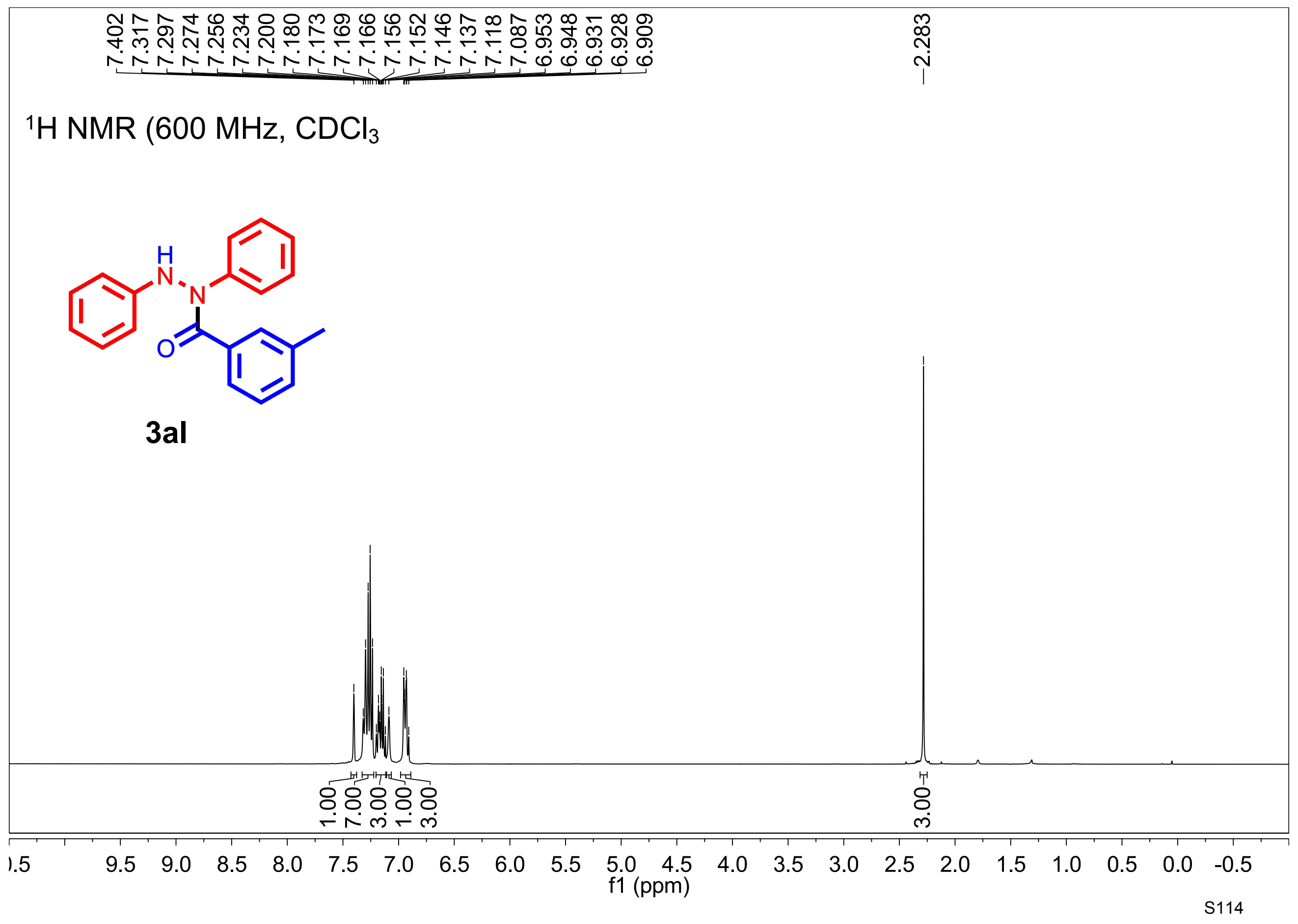




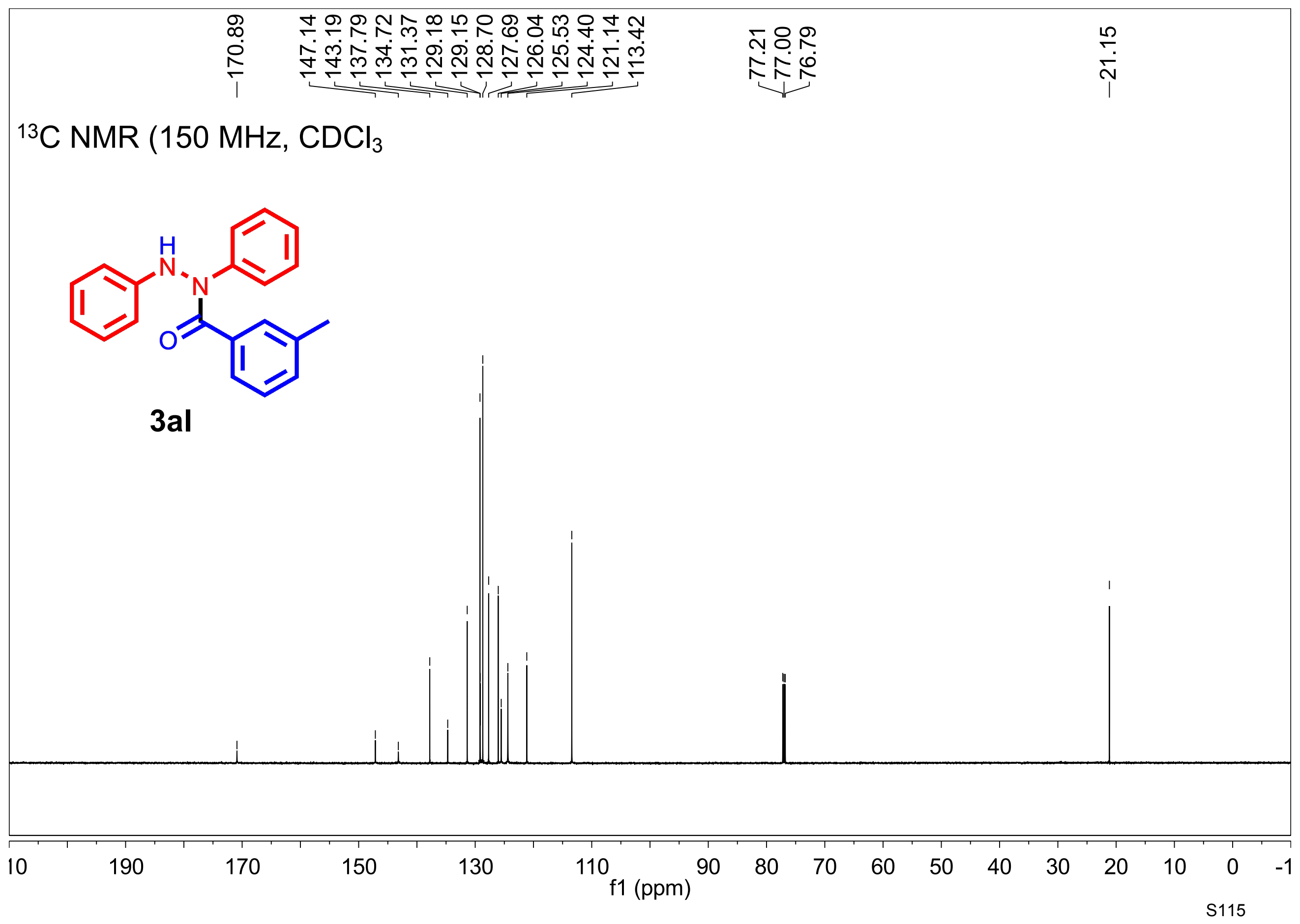


我

ம एँ

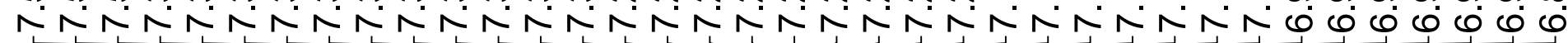

${ }^{1} \mathrm{H}$ NMR $\left(600 \mathrm{MHz}, \mathrm{CDCl}_{3}\right)$<smiles>O=C(c1cccc(Cl)c1)N(Nc1ccccc1)c1ccccc1</smiles>

3am

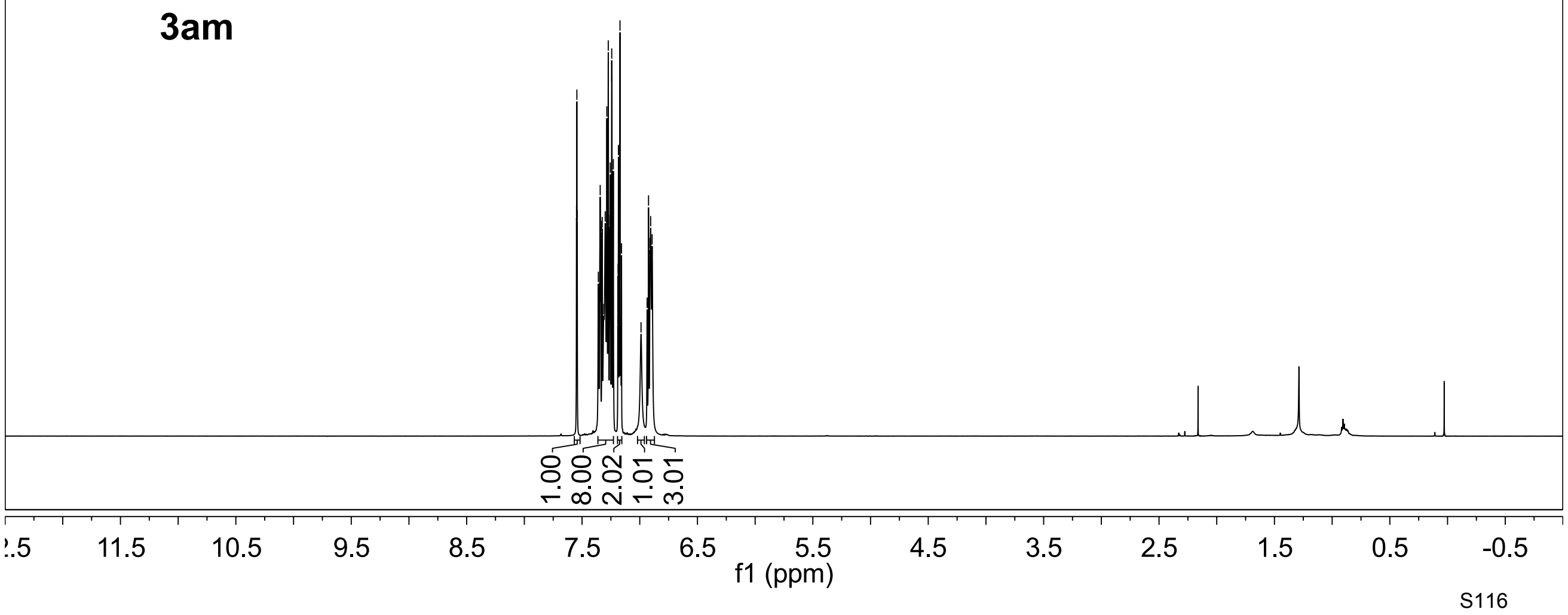




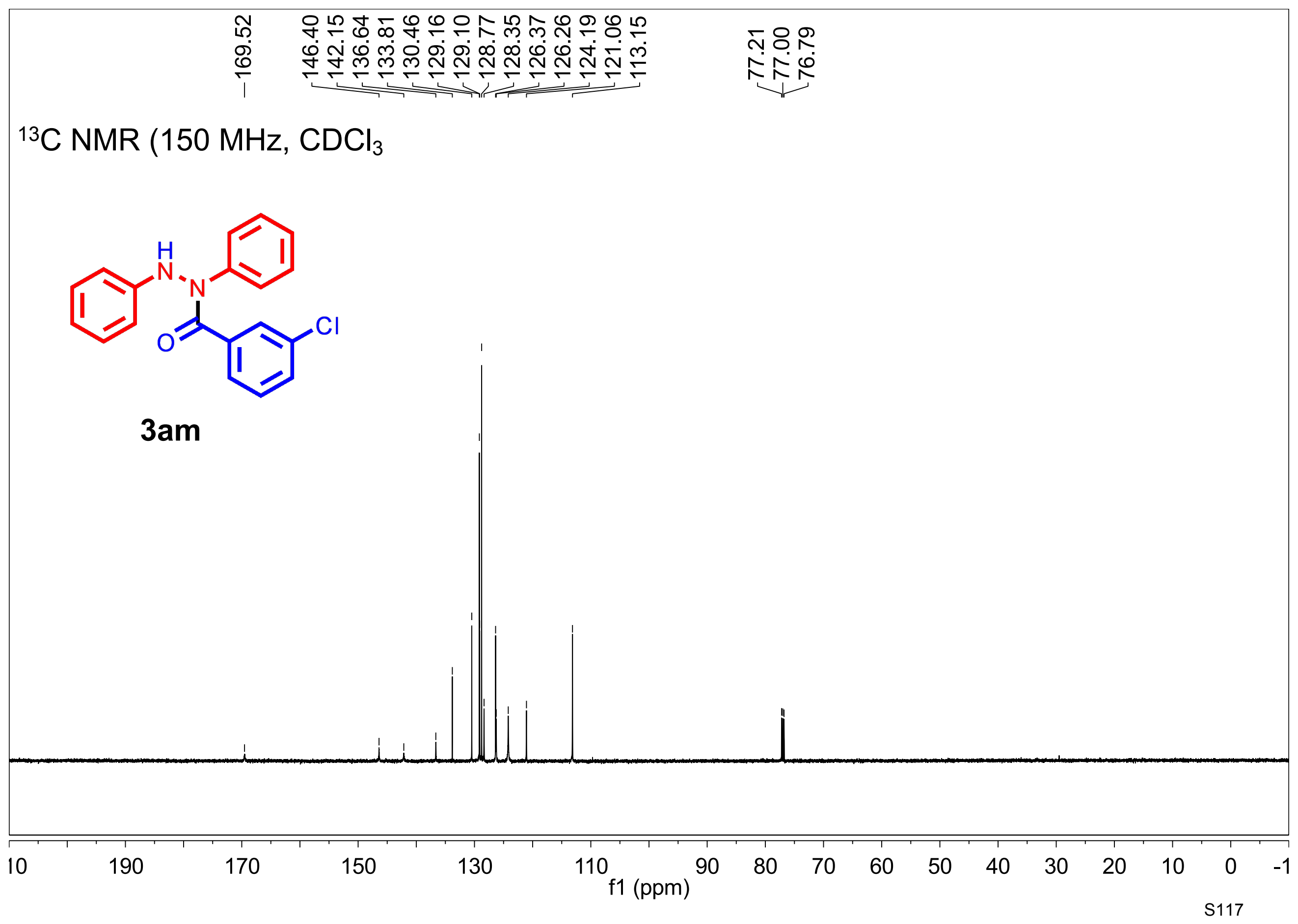




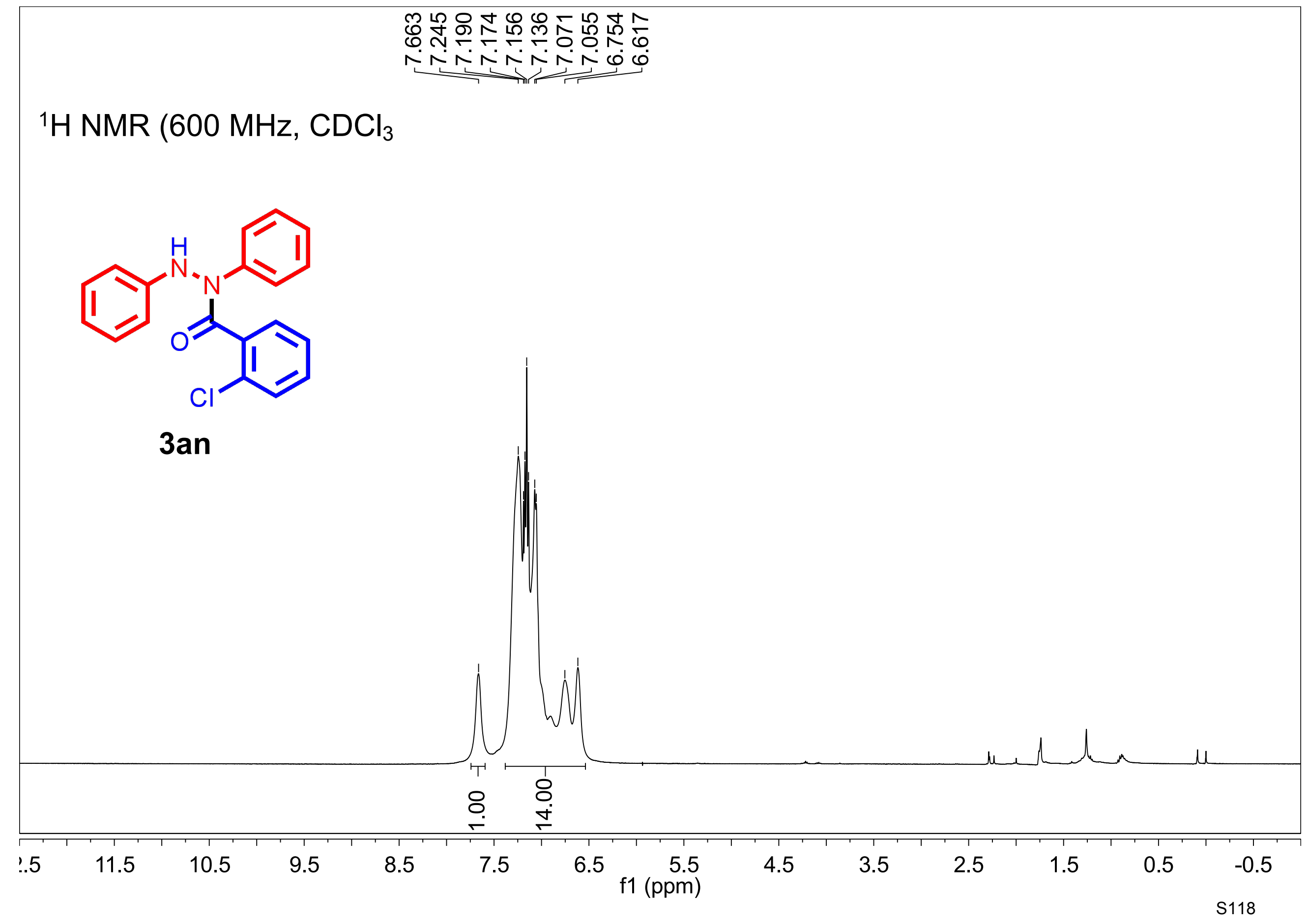




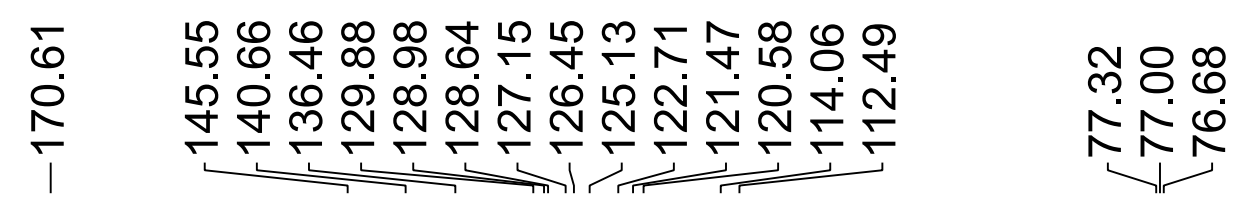

${ }^{13} \mathrm{C}$ NMR $\left(150 \mathrm{MHz}, \mathrm{CDCl}_{3}\right)$

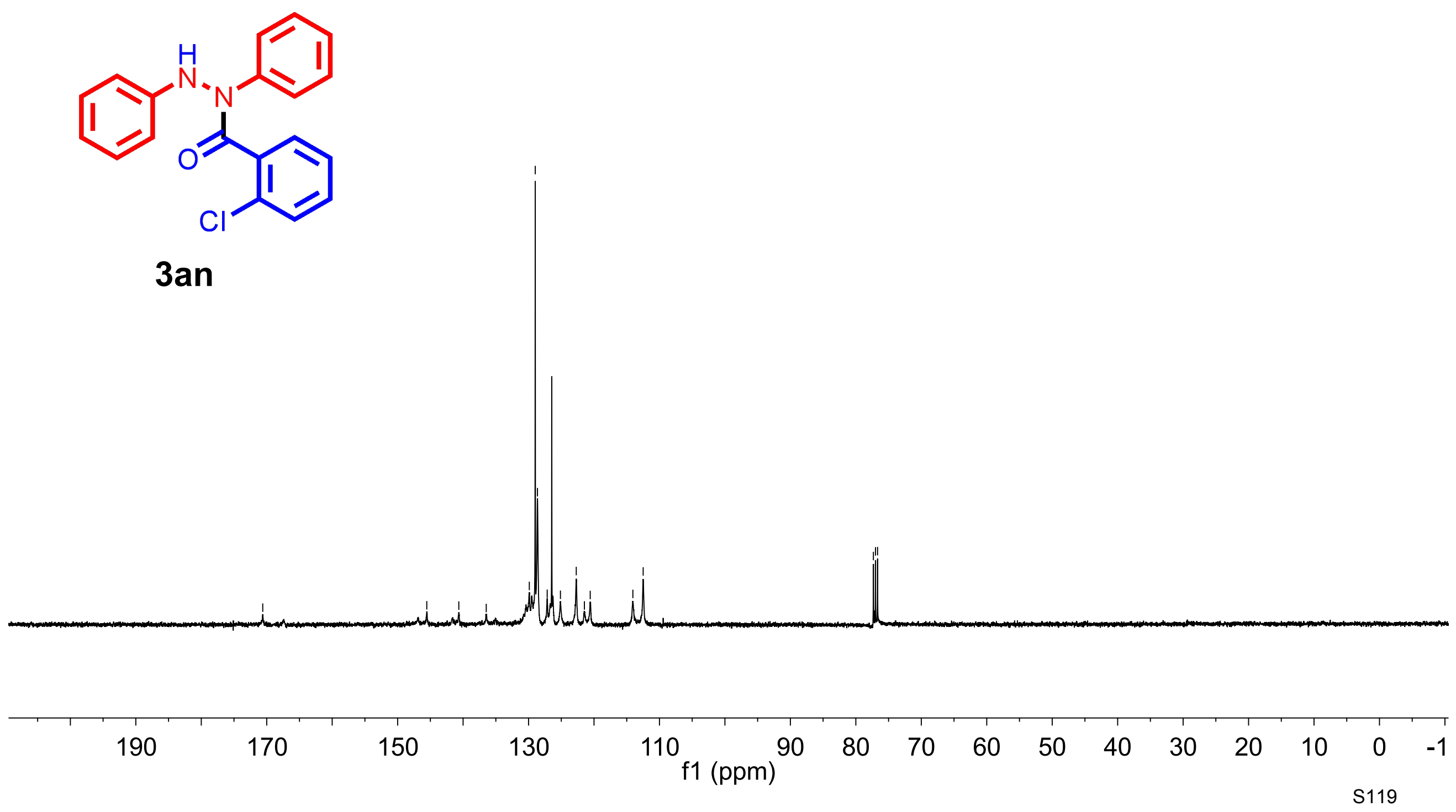


${ }^{1} \mathrm{H}$ NMR $\left(600 \mathrm{MHz}, \mathrm{CDCl}_{3}\right)$

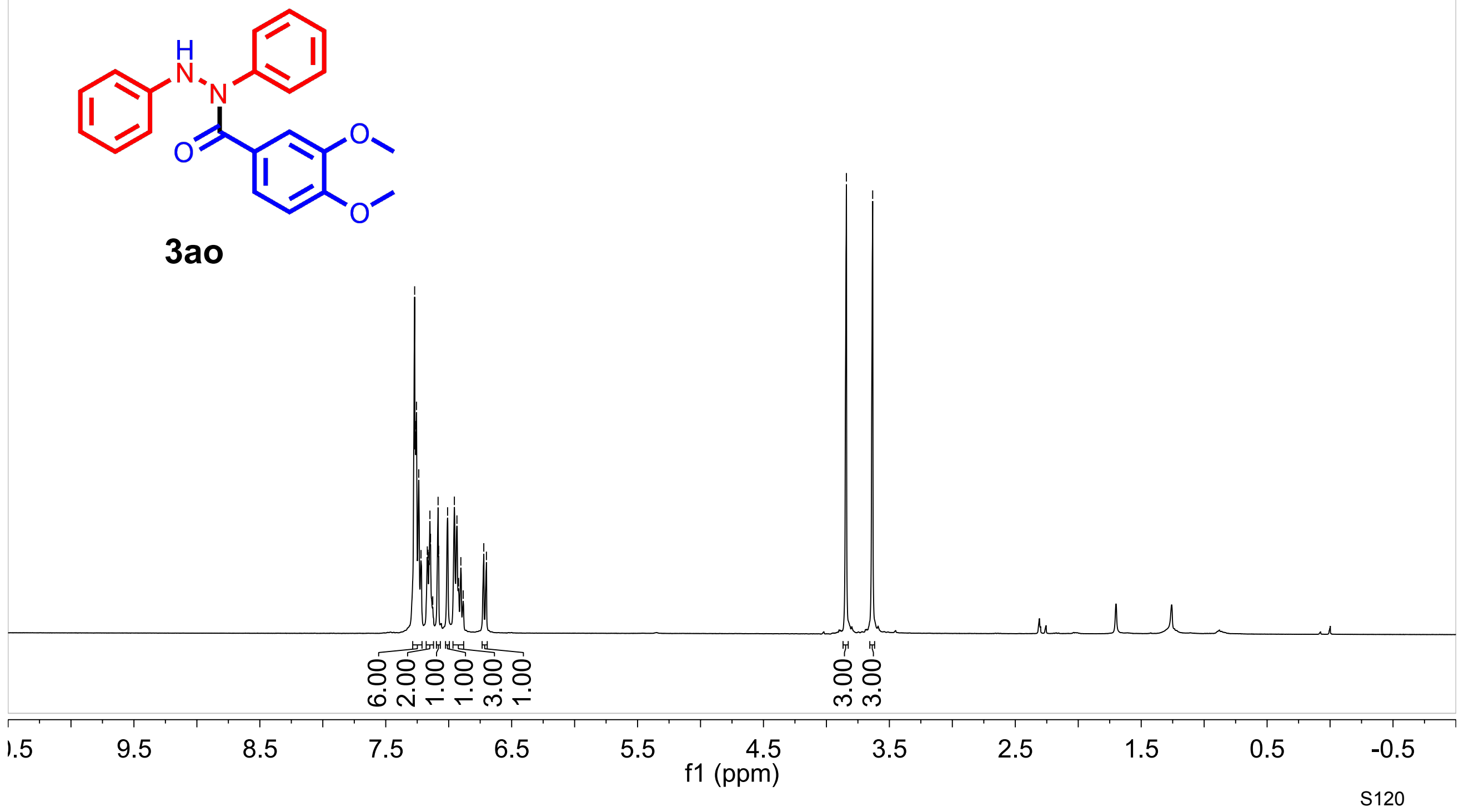




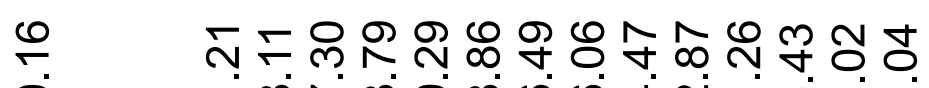

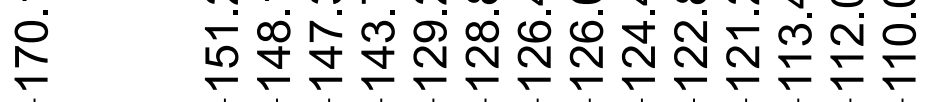

ㄷํㅇㅇำ

$\infty \widetilde{0}$

${ }^{13} \mathrm{C}$ NMR (150 $\left.\mathrm{MHz}, \mathrm{CDCl}_{3}\right)$

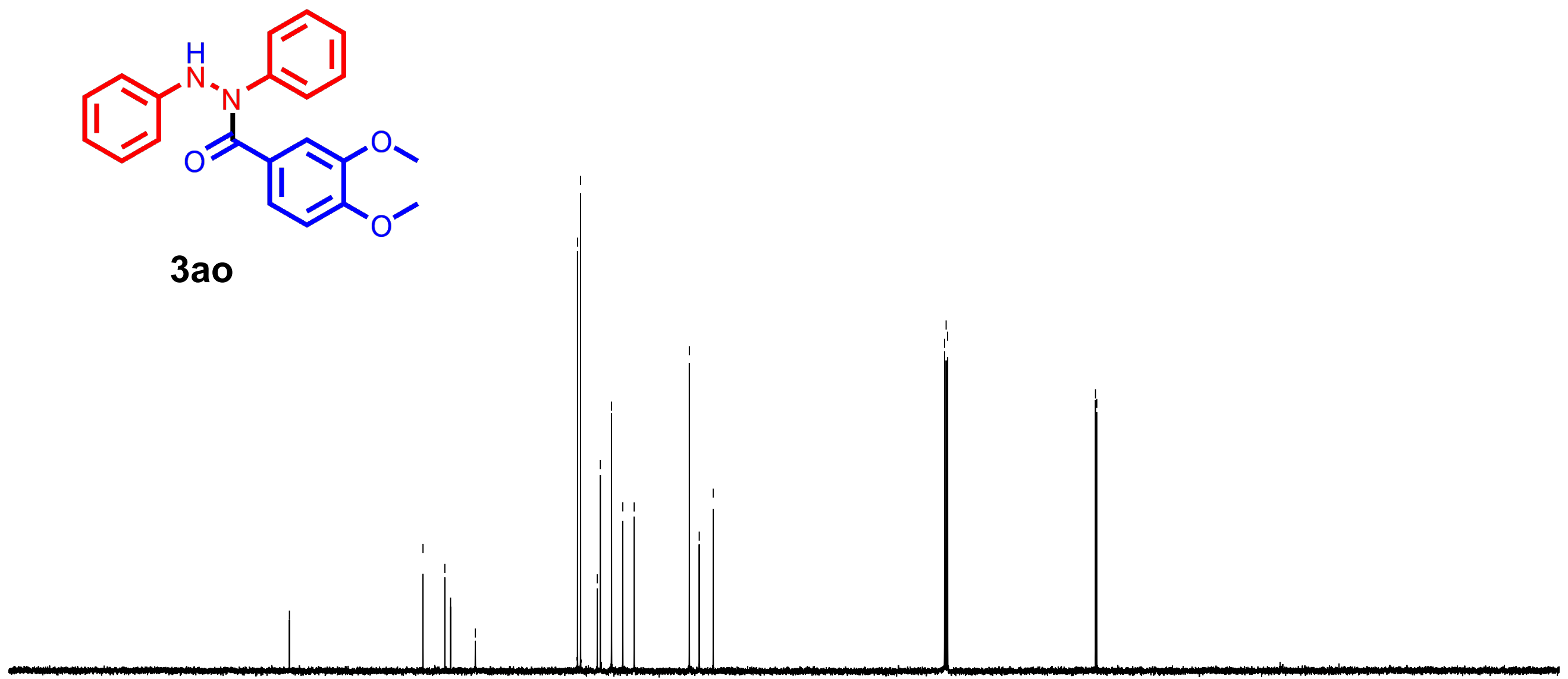




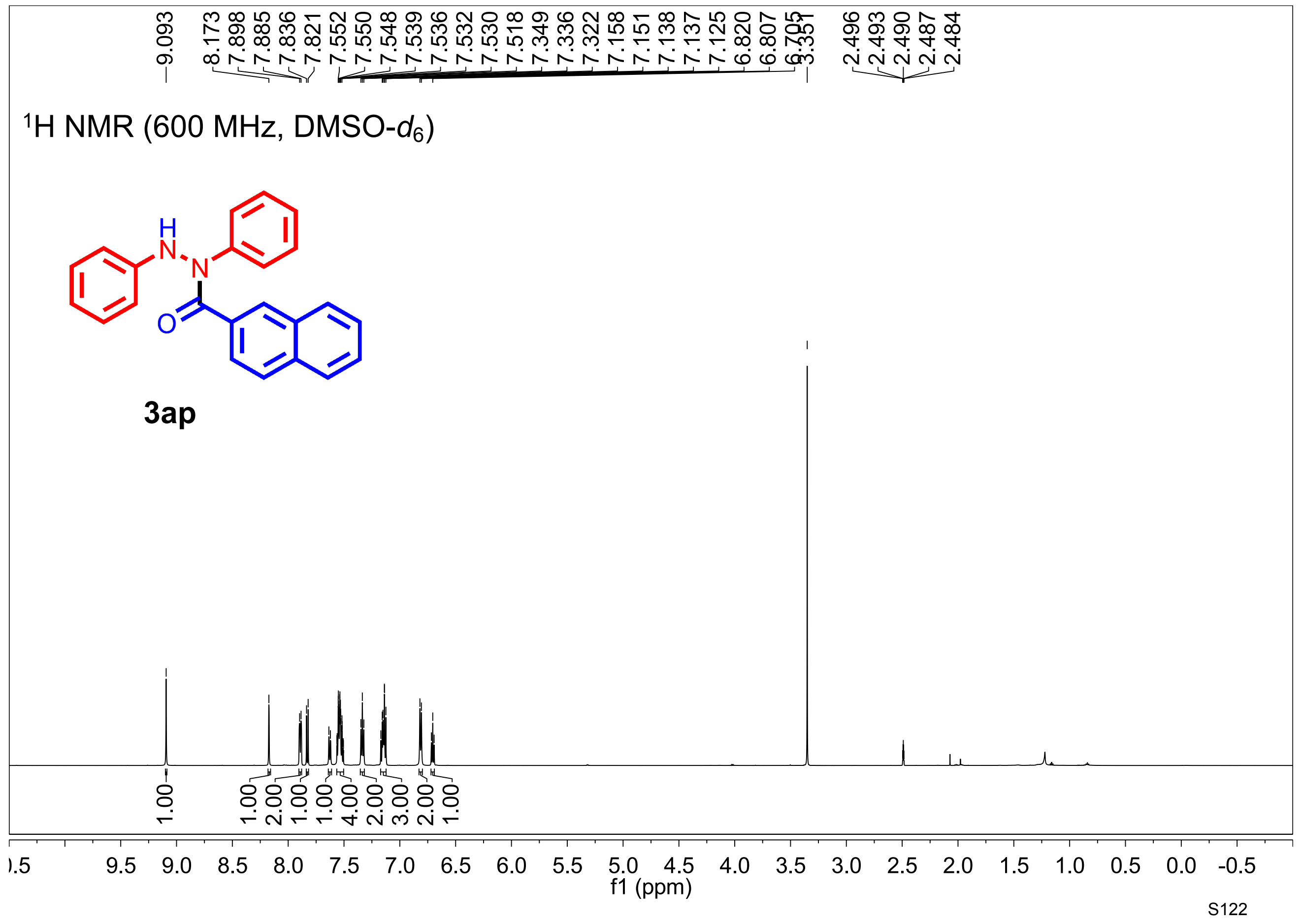


${ }^{13} \mathrm{C}$ NMR (150 MHz, DMSO- $\left.d_{6}\right)$

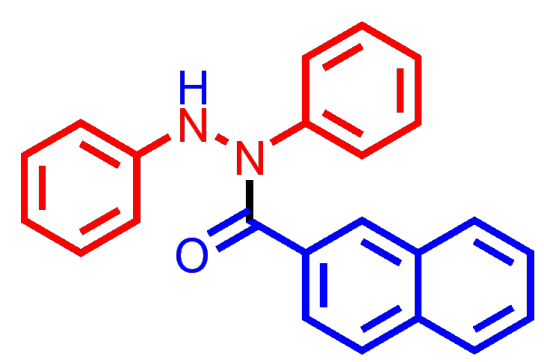

3ap

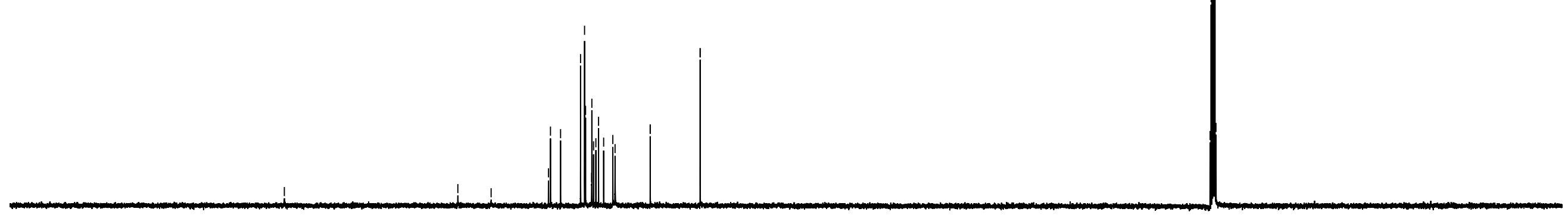




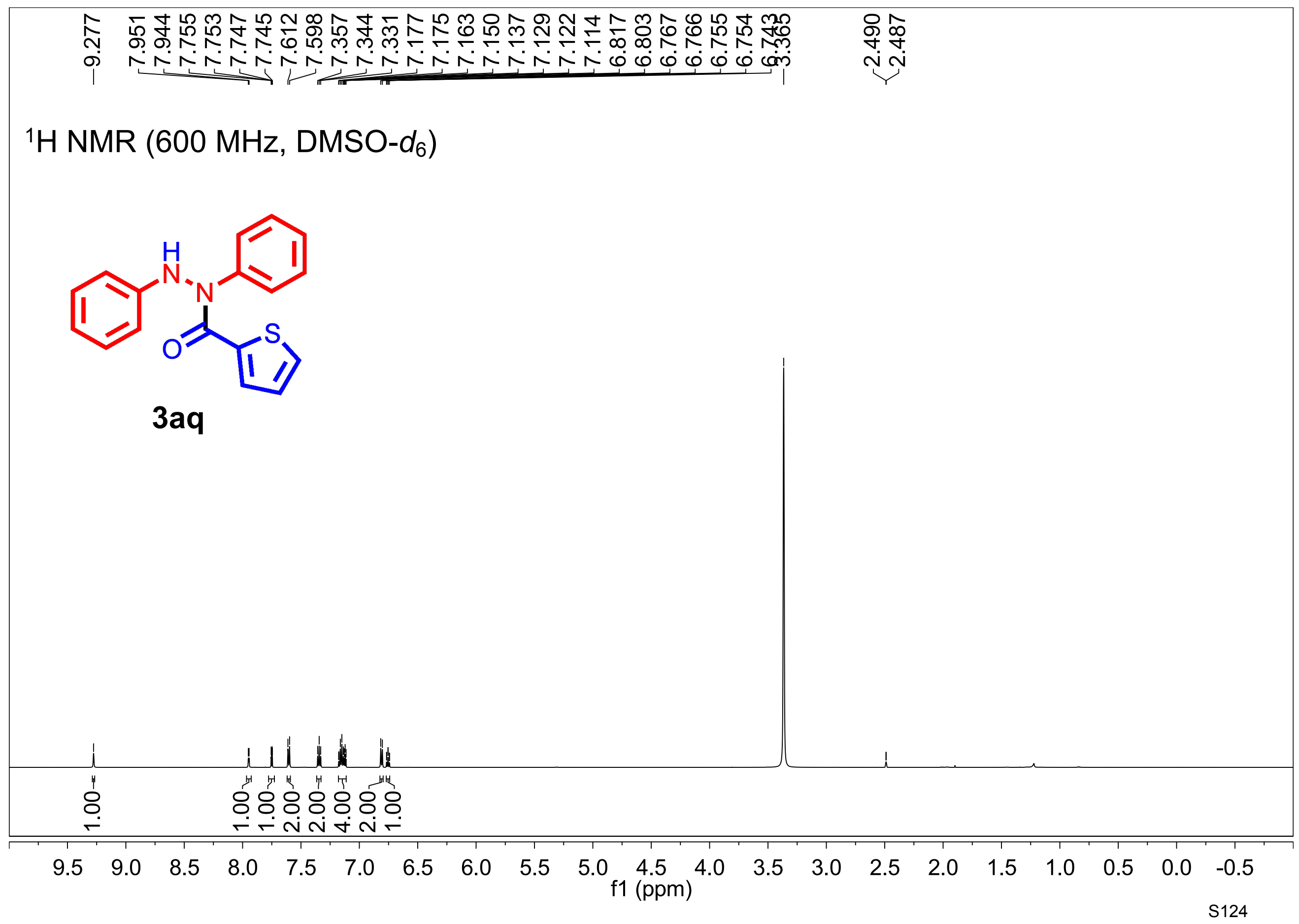




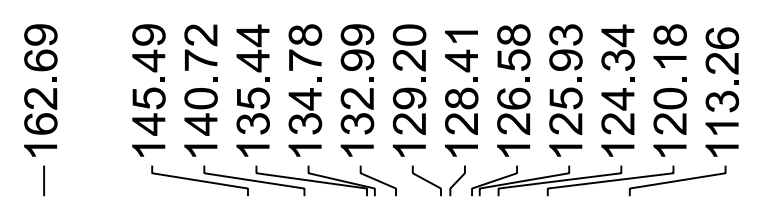

${ }^{13} \mathrm{C}$ NMR (150 MHz, DMSO- $\left.d_{6}\right)$

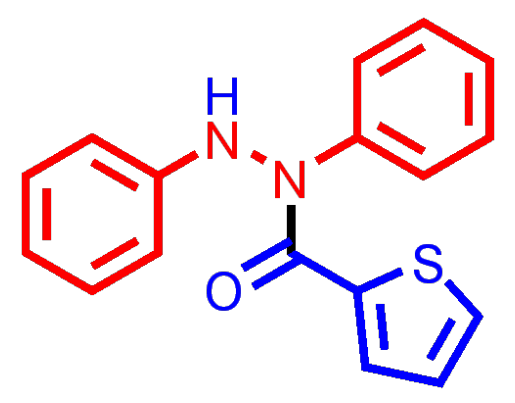

$3 a q$ 


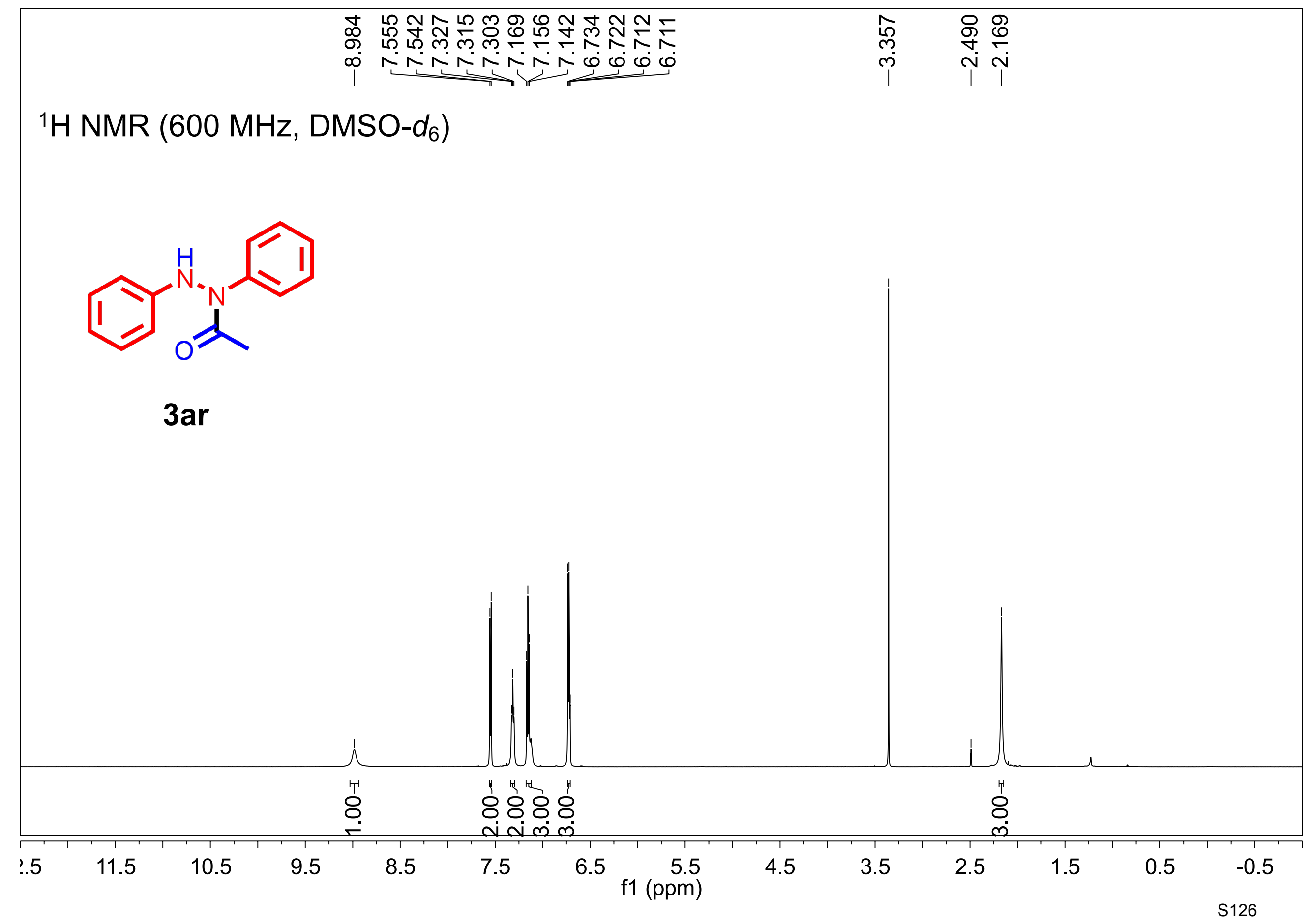




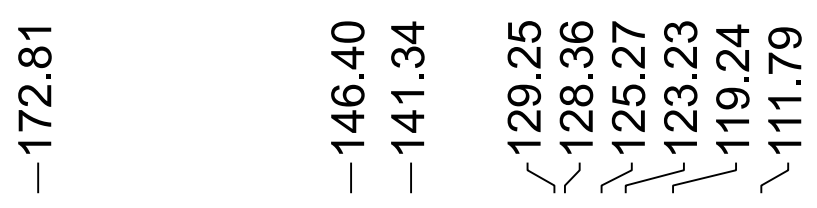

${ }^{13} \mathrm{C}$ NMR (150 MHz, DMSO- $d_{6}$ )

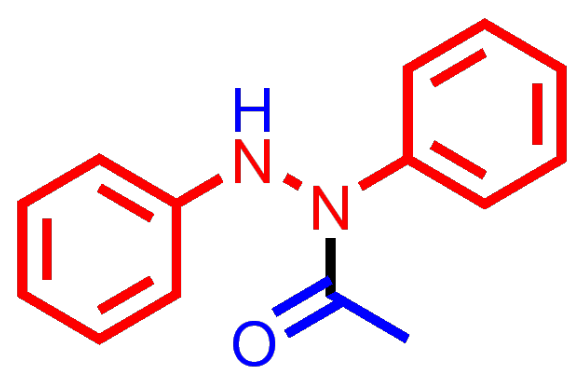

$3 a r$

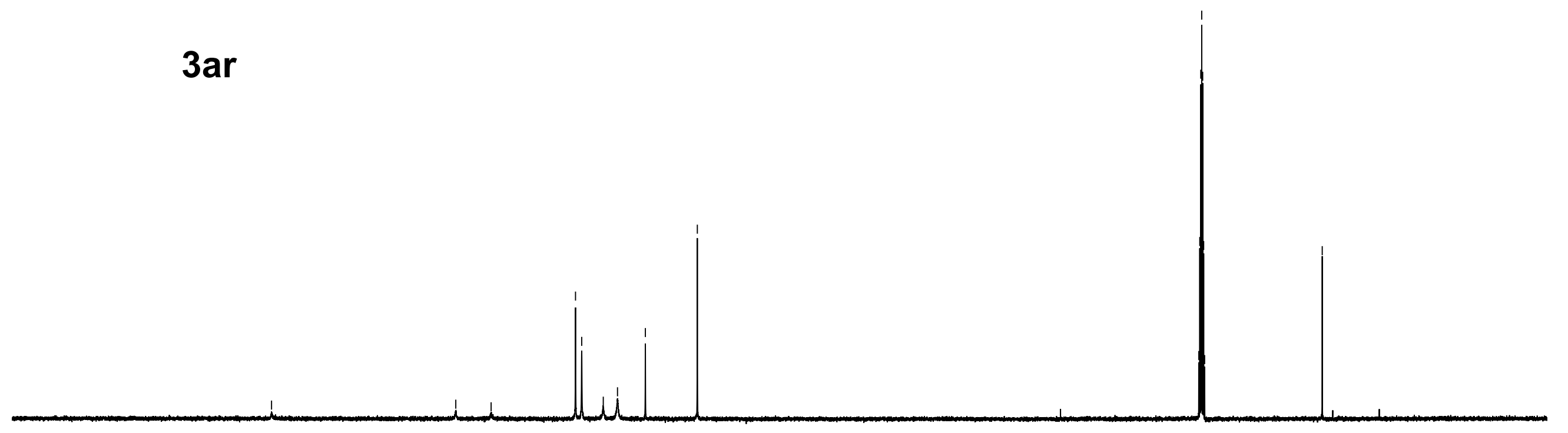




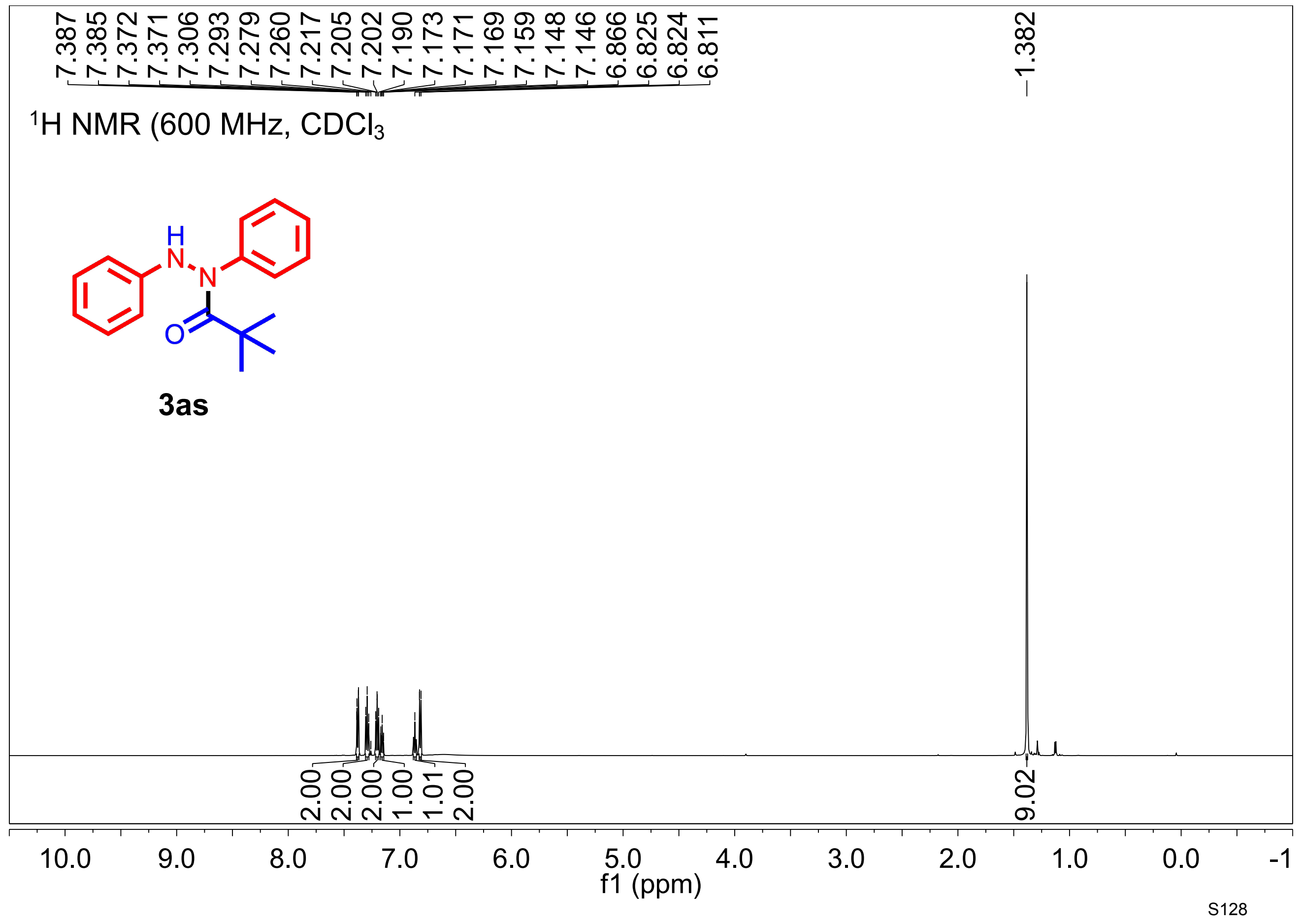




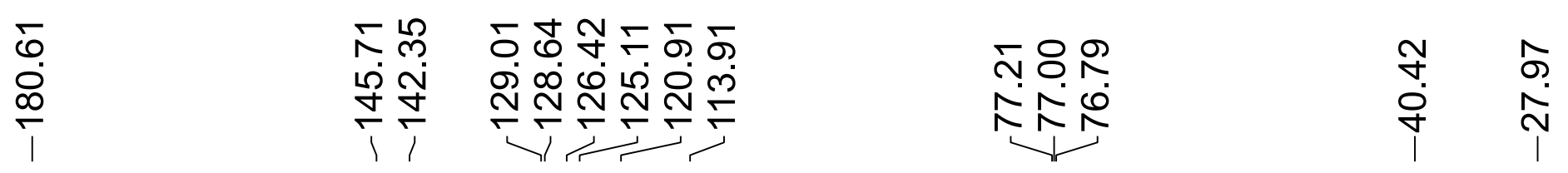

${ }^{13} \mathrm{C}$ NMR (150 $\left.\mathrm{MHz}, \mathrm{CDCl}_{3}\right)$

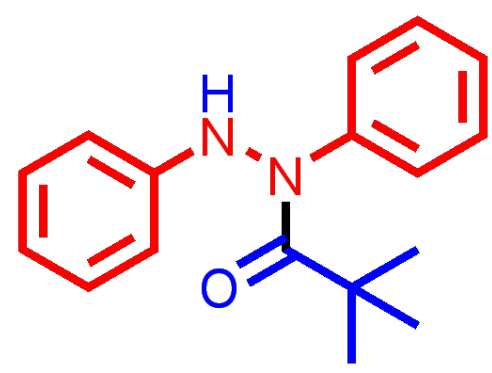

3as

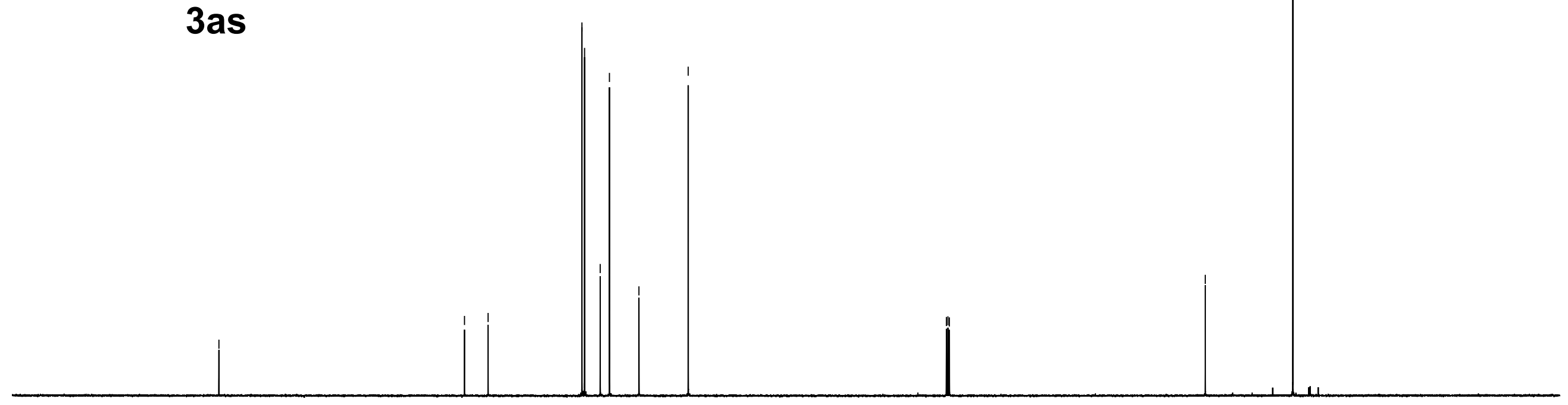


${ }^{1} \mathrm{H}$ NMR $\left(600 \mathrm{MHz}\right.$, DMSO- $\left.d_{6}\right)$

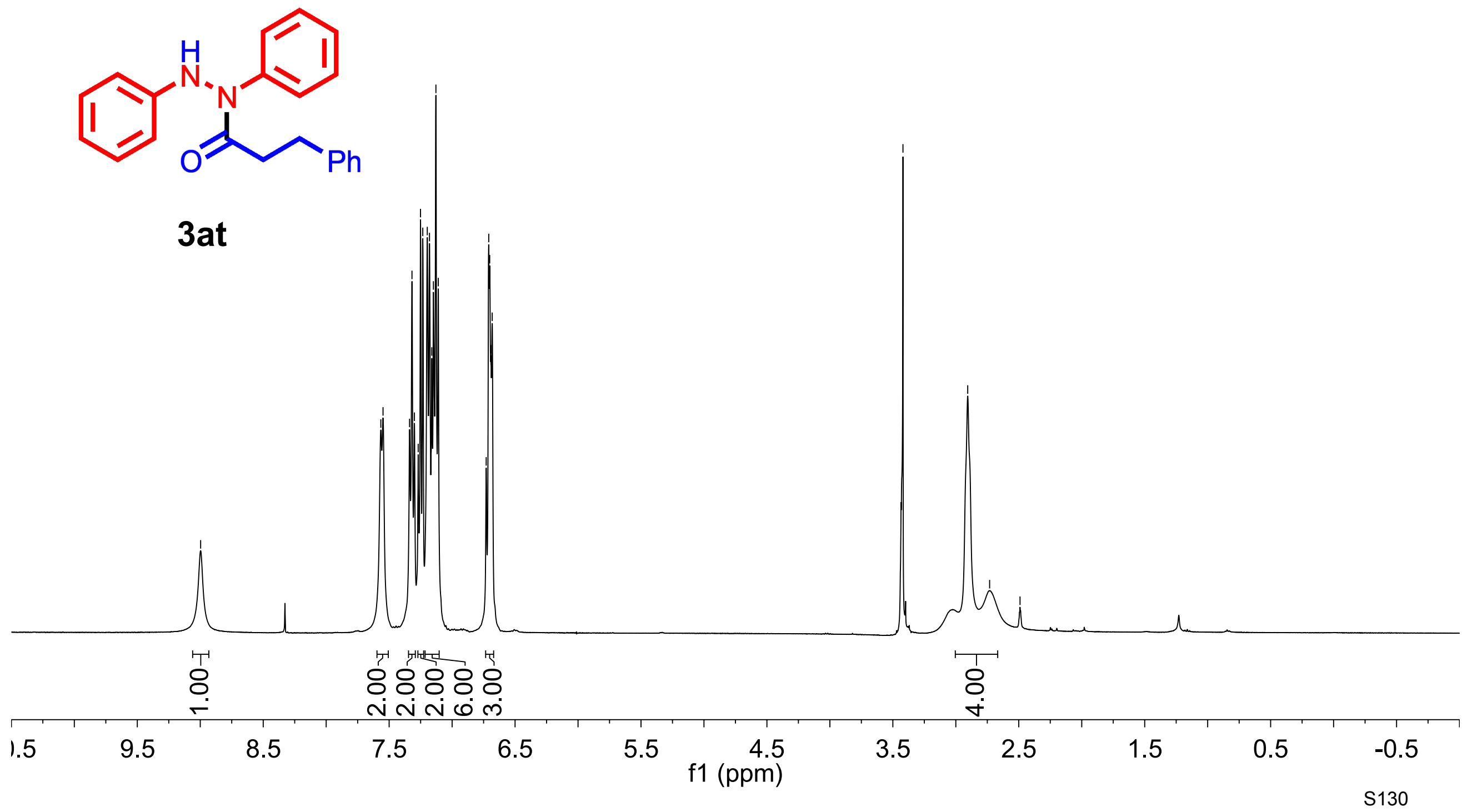




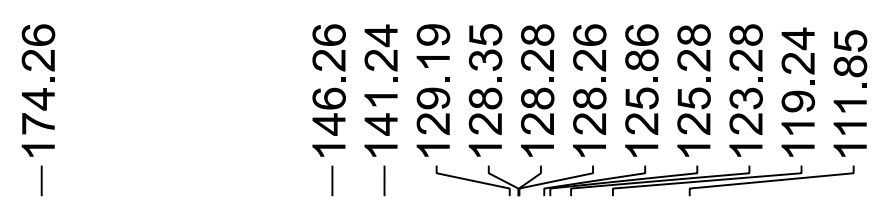

${ }^{13} \mathrm{C}$ NMR (150 MHz, DMSO- $\left.d_{6}\right)$

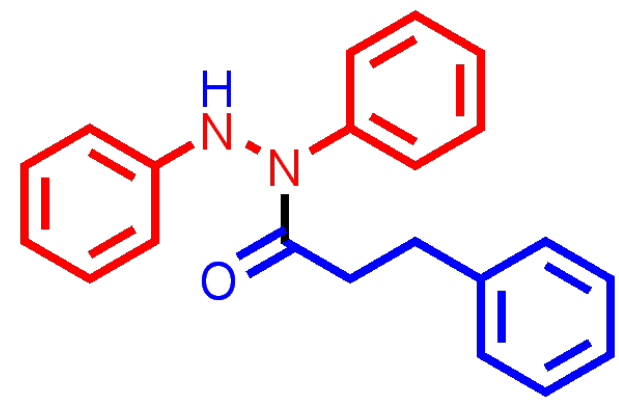

3at

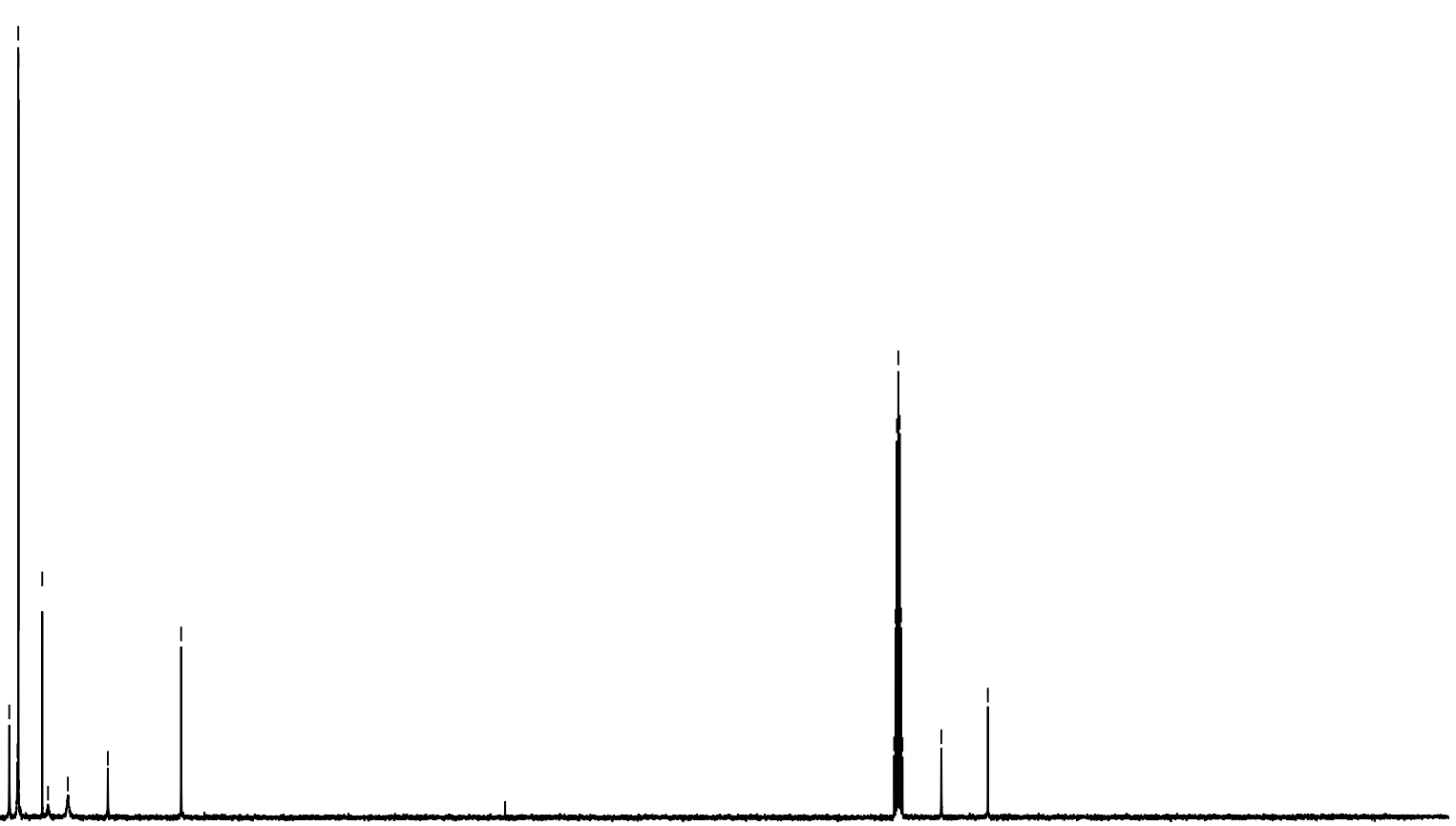

\begin{tabular}{lllllllllllllllllll}
\hline 230 & 210 & 190 & 170 & 150 & 130 & $\begin{array}{c}110 \\
\mathrm{f} 1(\mathrm{ppm})\end{array}$ & 90 & 80 & 70 & 60 & 50 & 40 & 30 & 20 & 10 & 0 &
\end{tabular}

